

\section{NOVEL BIOMEDICAL APPLICATIONS OF SUPPORTED LIPID BILAYERS}

Jasper van Weerd 
Members of the committee:

$\begin{array}{lll}\text { Chairman: } & \text { Prof.dr.ir. J.W.M. Hilgenkamp } & \text { (University of Twente) } \\ \text { Promotors: } & \text { Prof.dr.ir. P. Jonkheijm } & \text { (University of Twente) } \\ & \text { Prof.dr. M. Karperien } & \text { (University of Twente) } \\ \text { Members: } & \text { Prof.dr. R. Richter } & \text { (CIC biomaGUNE) } \\ & \text { Prof.dr. R.A. Bank } & \text { (University Medical Center Groningen) } \\ & \text { Prof.dr. W.T.S. Huck } & \text { (Radboud University) } \\ & \text { Prof.dr. M.M.A.E. Claessens } & \text { (University of Twente) } \\ & \text { Prof.dr. D.W. Grijpma } & \text { (University of Twente) } \\ & \text { Prof.dr. J.J.L.M. Cornelissen } & \text { (University of Twente) }\end{array}$

The research in this thesis was performed within the laboratories of the Laboratory of Bioinspired Molecular Engineering (LBME) group, the MESA ${ }^{+}$institute for Nanotechnology and the laboratories of the Developmental BioEngineering (DBE) group, MIRA institute for Biomedical technology and Technical Medicine of the Department of Science and Technology of the University of Twente. This thesis is part of NanoNextNL, a micro and nanotechnology innovation consortium of the Government of the Netherlands and 130 partners from academia and industry.

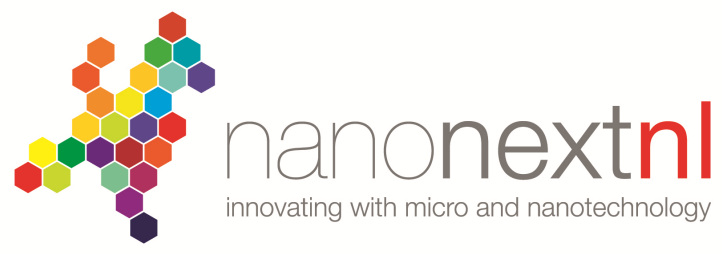

\section{NOVEL BIOMEDICAL APPLICATIONS OF SUPPORTED LIPID BILAYERS}

Copyright (C) 2015, Jasper van Weerd, Enschede, the Netherlands.

All rights reserved. No part of this thesis may be reproduced or transmitted in any form, by any means, electronic or mechanical, without prior written permission of the author.

ISBN: 978-90-365-3826-8

DOI: $10.3990 / 1.9789036538268$

Cover art: Kate Bakker and Jasper van Weerd

Printed by: Gildeprint - The Netherlands 


\title{
NOVEL BIOMEDICAL APPLICATIONS OF SUPPORTED LIPID BILAYERS
}

\section{DISSERTATION}

\author{
to obtain \\ the degree of doctor at the University of Twente, \\ on the authority of the rector magnificus, \\ Prof. dr. H. Brinksma, \\ on account of the decision of the graduation committee, \\ to be publicly defended
}

on Friday January 16,2015 , at $14.45 \mathrm{~h}$

by

Jasper van Weerd

Born on October 24, 1985

In Almelo, the Netherlands 
This dissertation has been approved by:

Promotors: $\quad$ Prof. dr. ir. P. Jonkheijm

Prof. dr. M. Karperien 
"No amount of experimentation can ever prove me right; a single experiment can prove me wrong."

- Albert Einstein - 



\section{Table of contents}

Chapter 1: Supported lipid bilayers for the generation of dynamic cell-material interfaces

1

1.1 Introduction 2

1.2 The natural cell membrane 3

1.3 Membrane components 5

1.4 Model membranes $\quad 7$

$\begin{array}{ll}\text { 1.4.1 SLB formation } & 7\end{array}$

$\begin{array}{ll}\text { 1.4.2 SLB substrates and architectures } & 10\end{array}$

$\begin{array}{ll}\text { 1.4.3 Patterning of SLBs } & 11\end{array}$

$\begin{array}{ll}1.5 \text { SLB analysis } & 15\end{array}$

$\begin{array}{ll}\text { 1.6 SLB characteristics } & 18\end{array}$

$\begin{array}{ll}\text { 1.6.1 Lipid phase behaviour and mobility } & 18\end{array}$

$\begin{array}{ll}\text { 1.6.2 Non-fouling nature of SLBs } & 20\end{array}$

1.6.3 Air instability of SLBs $\quad 22$

1.6.4 Leaflet symmetry and composition of SLBs 26

1.6.5 Barrier properties of SLBs 26

$\begin{array}{ll}1.7 \text { Introducing bio-functionality in SLBs } & 27\end{array}$

1.8 Bio-analytical applications of SLBs 31

1.8.1 Membrane electrophoresis $\quad 31$

1.8.2 Studying membranes processes using SLBs 33

1.8.3 Biosensing and analysis of membrane proteins 34

1.8.4 Cytoskeletal organization 36

$\begin{array}{ll}\text { 1.8.5 Membrane repair } & 36\end{array}$

1.9 SLBs as an interface to cells 37

$\begin{array}{ll}\text { 1.9.1 Mimicking cell-cell contacts } & 37\end{array}$

1.9.2 Supporting cell differentiation on SLBs 38

1.9.3 3D presentation of ligands using SLBs $\quad 40$

1.9.4 Mimicking cell-ECM contacts using peptide-modified SLBs 41

1.9.5 Mimicking cell-ECM contacts by decorating SLBs with

ECM proteins $\quad 42$ 
1.9.6 Mimicking cell-ECM contacts using a protein network for peptide presentation $\quad 45$

1.9.7 Cell adhesion on charged SLBs $\quad 46$

$\begin{array}{ll}1.10 & \text { Use of gradient SLBs for cell studies }\end{array}$

1.10.1 Screening of RGD-lipid density in SLBs 46

1.10.2 Use of locked-in SLB gradients for cell studies $\quad 48$

1.11 Use of patterned SLBs in cell studies $\quad 48$

1.11.1 Use of micro-contact printed patterned SLBs 48

$\begin{array}{ll}\text { 1.11.2 Use of particle-based SLB systems } & 50\end{array}$

1.11.3 Use of metal corrals in SLB studies 52

1.12 Towards in vivo application of SLBs $\quad 57$

1.12.1 Nanoparticle supported lipid bilayers $\quad 57$

$\begin{array}{ll}\text { 1.12.2 Lipid mimetic coating } & 58\end{array}$

$\begin{array}{ll}\text { 1.12.3 Biomaterial supported SLBs } & 60\end{array}$

$\begin{array}{ll}\text { 1.13 Scope and outline of the thesis } & 60\end{array}$

$\begin{array}{ll}1.14 \text { References } & 62\end{array}$

Chapter 2: On-Chip Electrophoresis in Supported Lipid Bilayer Membranes Achieved Using Low Potentials 69

$\begin{array}{ll}2.1 \text { Introduction } & 70\end{array}$

2.2 Results and discussion $\quad 71$

$\begin{array}{ll}2.3 \text { Conclusions } & 78\end{array}$

$\begin{array}{ll}2.4 \text { Experimental section } & \mathbf{7 8}\end{array}$

$\begin{array}{ll}2.4 .1 \text { Materials } & 78\end{array}$

$\begin{array}{ll}2.4 .2 \text { Methods } & 78\end{array}$

$\begin{array}{ll}2.5 \text { References } & 81\end{array}$ 
Chapter 3: Locked-in biomimetic surface gradients that are tuneable in size, density and functionalization

3.1 Introduction

3.2 Results and discussion

3.3 Conclusions

3.4 Acknowledgements

3.5 Experimental section 92

3.5.1 Materials

3.5.2 Methods 92

3.6 References

95

Chapter 4: Probing the threshold in bacteria-mannose recognition using SLB gradients

4.1 Introduction 98

4.2 Results and discussion 99

4.3 Conclusions 107

4.4 Acknowledgements 107

4.5 Experimental section 107

4.5.1 Materials 107

4.5.2 Methods 107

4.6 References 110

4.7 Supporting information

Chapter 5: Tuning cell adhesion, spreading and matrix formation of cells in vitro using SLBs

5.2 Results and discussion

5.2.1 Human primary chondrocytes cultured on SLBs

5.2.2 KS483 matrix deposition 
5.4 Experimental section $\quad \mathbf{1 2 4}$

$\begin{array}{ll}\text { 5.4.1 Materials } & 124\end{array}$

$\begin{array}{ll}\text { 5.4.2 Methods } & 125\end{array}$

$\begin{array}{ll}5.5 \text { References } & 127\end{array}$

$\begin{array}{ll}\text { 5.6 Supporting Information } & 128\end{array}$

Chapter 6: Biomaterial-supported lipid bilayer as a tuneable cell surface

6.1 Introduction

132

6.2 Results and discussion 133

6.2.1 Biomaterial activation 134

6.2.2 Biomaterial SLB formation 136

6.2.3 Mesenchymal stem cell culture on BSLBs $\quad 140$

$\begin{array}{ll}6.3 \text { Conclusions } & 143\end{array}$

$\begin{array}{ll}\text { 6.4 Acknowledgements } & 144\end{array}$

$\begin{array}{ll}\text { 6.5 Experimental section } & 144\end{array}$

$\begin{array}{ll}\text { 6.5.1 Materials } & 144\end{array}$

$\begin{array}{ll}\text { 6.5.2 Methods } & 145\end{array}$

$\begin{array}{lr}6.6 \text { References } & 149\end{array}$

$\begin{array}{ll}\text { 6.7 Supporting information } & 150\end{array}$

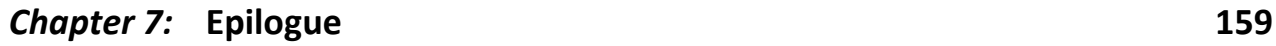

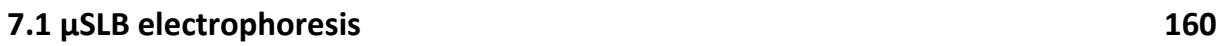

$\begin{array}{ll}7.2 \text { Locked-in gradients } & 160\end{array}$

$\begin{array}{ll}\text { 7.3 Directing cell fate using SLBs } & 161\end{array}$

$\begin{array}{ll}\text { 7.4 SLBs and supramolecular assemblies } & 162\end{array}$

$\begin{array}{ll}7.5 \text { References } & 163\end{array}$

$\begin{array}{ll}\text { Summary } & 165\end{array}$ 


\section{Chapter 1}

\section{Supported lipid bilayers for the generation of dynamic cell-material interfaces*}

Herein we present the state-of-the-art in supported lipid bilayers (SLB) based technology. The fabrication, analysis, characteristics and modification of SLBS are described in great detail. Numerous strategies are described to form SLBs on different substrates and the means to patterns them. The use of SLBs as model membranes for the study of membrane organization and membrane processes in vitro is highlighted. In addition, the use of SLBs as a substratum for cell analysis is presented where we discriminate between cell-cell and cell-extracellular matrix (ECM) mimicry. Finally the potential for the in vivo application of SLBs is demonstrated and an outlook of the research described in this thesis is presented.

\footnotetext{
"Part of this Chapter has been published in: J. Brinkmann, E. Cavatorta, S. Sankaran, B. Schmidt, J. van Weerd and P. Jonkheijm; Chem. Soc. Rev. (2014) vol. 43, p.4449-4469

J. van Weerd, M. Karperien and P. Jonkheijm (2014) manuscript in preparation
} 


\subsection{Introduction}

In nature, virtually all cells make use of a membrane to separate and shield its components from the outside environment. The cellular membrane structure is based on a two-ply sheet of lipid molecules that are highly dynamic, ordered and decorated with a wide range of biomolecules in a spatiotemporal controlled fashion. This complex interface is crucial for cell function in e.g. transport and complex intracellular signalling processes. ${ }^{1-3}$ Capturing the complexity of this interface between the cell's interior and exterior is a daunting task and up to this day it remains challenging to assess in living cells despite impressive progress in spectroscopic techniques. To further our understanding and study and mimic these interfaces, synthetic architecture analogues that are capable of dynamically presenting biological ligands are investigated.

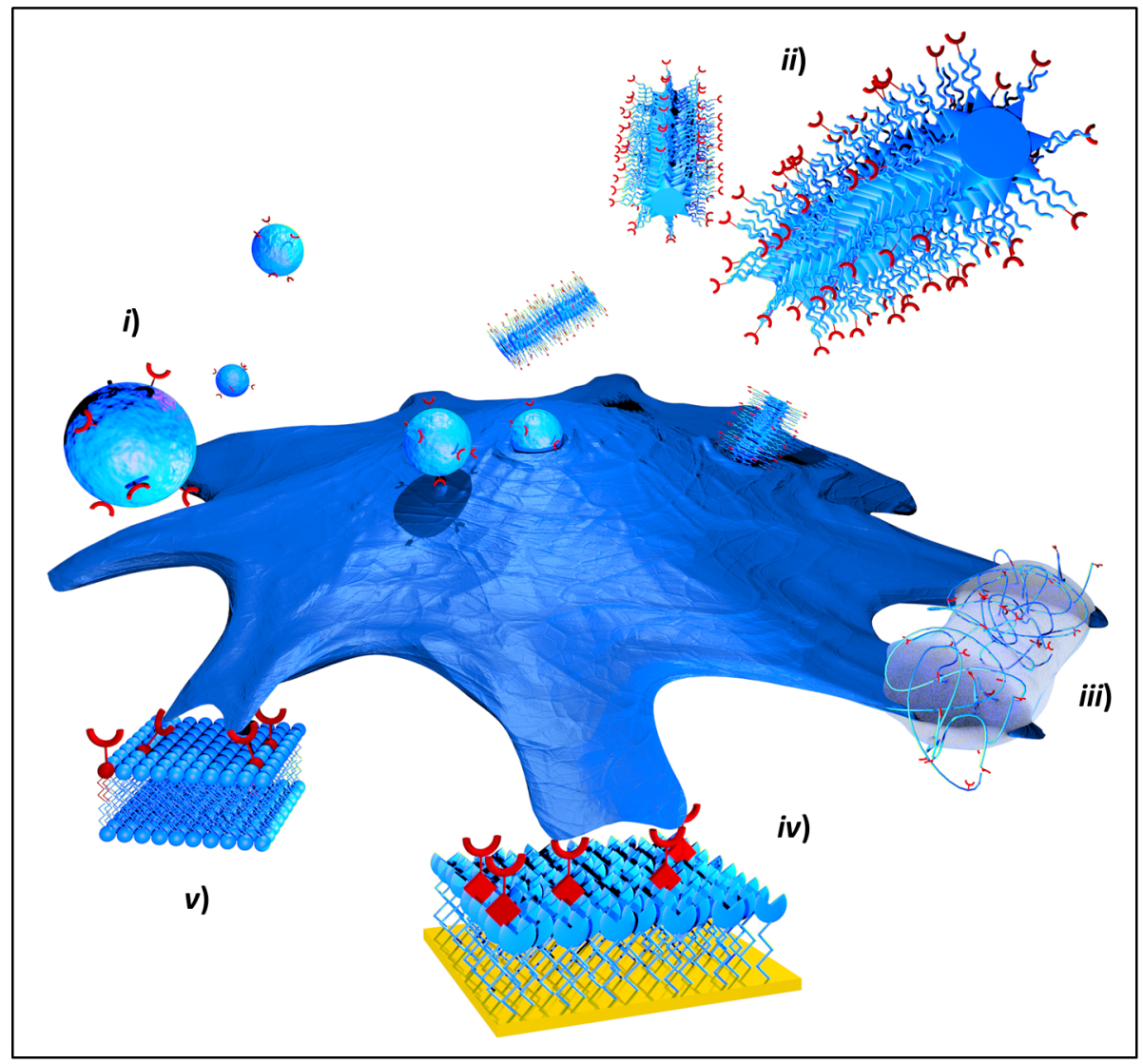

Figure 1.1: Overview of various dynamic biological architectures; i) vesicles, ii) self-assembled amphiphiles, iii) hydrogels, iv) self-assembled monolayers and $\boldsymbol{v}$ ) lipid bilayers each of which can be designed to dynamically present ligands (red) required for specific engagement with cells. Reprinted with permission. ${ }^{4}$ Copyright 2014 , Royal Society of Chemistry. 
Here, control over ligand characteristics, like mobility, density and presentation, are paramount to recapitulate the complexity of the cell membrane and associated processes to understand and steer biological function. A variety of dynamic biological architectures have been developed to investigate cell interaction (Figure 1.1). ${ }^{4}$ Generating such architectures often combines supramolecular assembly with fabrication techniques, adaptive chemistry, modern molecular biology and imaging techniques for their analysis. In addition, the use of reversible chemical strategies is a rewarding task in the development of functional materials and devices. Making use of bottom-up self-assembly, ligand distribution at predefined locations can be modulated.

For an overview of the various strategies that have been used to fabricate dynamic biological architectures for exploring their interaction with cells, such as protein/peptide scaffolds, amphiphile nanostructures, dynamic self-assembled monolayers and DNA-based nanostructures the reader is referred to a recent review by Jonkheijm and co-workers. ${ }^{4}$ In this review, the focus lies on providing the reader with a comprehensive overview of the work that has been performed on model cell membranes and in particular supported lipid bilayers (SLB). We start by recapitulating key characteristics of natural cell membranes and its constituents and then continue by summarizing the various methods that exist to mimic this highly complex, multi-molecular biological structure. Herein, formation and modification of model membranes and characterization strategies are discussed. The in vitro use of model membranes for studying membrane composition to increase our understanding of cellular processes is described. Also, efforts to mimic cell-cell and cellextracellular matrix (ECM) contacts using model membranes are given. Finally, the potential of exploiting SLBs for in vivo applications is demonstrated.

\subsection{The natural cell membrane}

In vivo, the cell membrane forms a physical barrier between the intra- and extracellular compartments. It plays a critical role in cell-cell communication, signal transduction and transport (Figure 1.2). The cell membrane is a highly complex system consisting of a phospholipid bilayer in which a variety of proteins is embedded or anchored. This system was described by Singer and Nicholson in 1972 using the 'fluid mosaic model'. ${ }^{5}$ In this model the lipid bilayer is considered as a two dimensional liquid in which proteins and lipids can move freely. It has been found that natural cell membranes are equipped with a large variety of lipids and proteins with a high degree of lateral dynamicity, flexibility and a high level of complexity and ordering. ${ }^{6,7}$ As a result, the 'fluid mosaic model' has been updated to include variable patchiness, variable thickness and higher protein occupancy than was previously considered. ${ }^{6}$ Apart from lateral dynamicity, lipid translocation between leaflets is crucial for biological function. ${ }^{8,9}$ The lipid 'flip-flop' is mediated by specific enzymes and membrane constraints, trapping lipids in one of the leaflets. ${ }^{10}$ The 
resulting membrane asymmetry is essential in, for example, cell fusion, coagulation processes and apoptosis. ${ }^{11}$

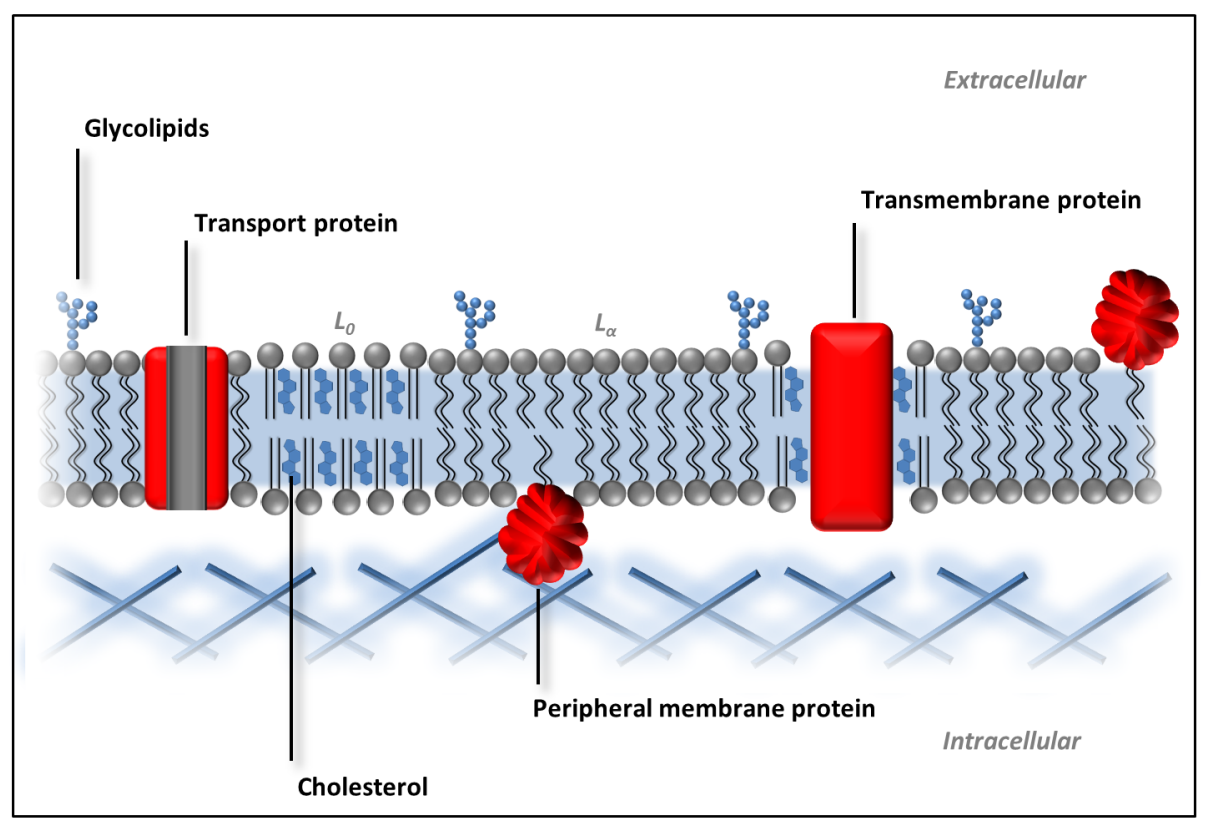

Figure 1.2: illustration of the natural cell membrane and its components.

Early findings that suggested the existence of membrane patchiness came from the observations of detergent resistant membranes (DRM). Using non-ionic detergents, like Triton X-100 or CHAPS, cell membranes do not fully solubilize and liquid-ordered DRM domains were isolated on sucrose gradients. ${ }^{12,13}$ These membrane patches are often referred to as lipid rafts comprising of liquid-ordered $\left(L_{0}\right)$ domains. ${ }^{14}$ These raft domains are considered as sterol-dependent states where lipid acyl chains are highly extended and packed tightly in the presence of cholesterol. This patchiness allows the cell to laterally segregate and organize its membrane components ${ }^{15}$ resulting in clustering of associated proteins like surface receptors. ${ }^{16}$

A different type of cell membrane domain is characterized by flask-shaped invaginations of the cell membrane. These so-called caveolae are involved in cell signalling and transport across the membrane. Caveolae are known to regulate vascular reactivity and blood pressure. In vivo caveolae function is mediated by coat proteins (caveolins) and support/ adapter proteins (cavins). ${ }^{17}$ The lipid composition of the cell membrane dictates the interactions between the membrane and the cytoskeleton. Sterol-dependent domains, 
rafts and caveolae, are essential for the coupling of the membrane with the-cytoskeleton. The cytoskeleton, which are made up of actin filaments, and the cell membrane are closely related and can influence the regulation of cellular biomechanical properties. As such, differential membrane domains, like rafts and caveolae, are now highly recognized to be responsible for imparting lateral heterogeneity to cell membranes and essential for biological function. The regulation and formation of these membrane domains is driven by lipid-lipid, lipid-protein and protein-protein interactions as well as interaction of cytoskeletal domains compounding cell membrane complexity. ${ }^{15,18,19}$

\subsection{Membrane components}

Apart from a vast array of proteins, over 1,000 different lipids have been suggested to be present in any eukaryotic cell (Figure 1.3). ${ }^{20}$ Based on their chemical structures, three main categories can defined, glycerophospholipids, sphingolipids and sterols. Both synthetic and natural lipids have been studied and prepared. For example glycerophospholipids are described with varying head-group groups on the $s n-3$ position of the glycerol backbone resulting in phosphatidylcholine (PC), phosphatidylethanolamine $(\mathrm{PE})$, phosphatidylserine (PS), phosphatidylglycerol (PG), phosphatidylinositol (PI) and phosphaditic acid (PA). Apart from varying in the head-group composition, the acyl chains at positions $s n-1$ and $s n-2$ can be different in length and degree of saturation (number of double bounds) and are crucial for determining the lipid phase behaviour. Apart from glycerol, other lipid backbones are found such as ester, alkyl ether or alkenyl ether bonds. ${ }^{7}$ PC is the most abundant lipid in vivo (> 50\%). Often one cis-unsaturated acyl tail is present to retain fluidity and lateral mobility at room temperature. ${ }^{21}$ Sphingomyelin and glycosphingolipids, which have a ceramide backbone, are the most common sphingolipids in eukaryotic cells. Sphingomyelins contain a saturated (or trans-unsaturated) hydrophobic tail and are able to form taller and narrower cylinders than PC of the same chain length resulting in a denser packing of lipids with limited lateral mobility. Interestingly, these tightly-packed structures can be fluidized by the presence of sterols. ${ }^{22}$ Cholesterol, the main sterol in mammalian cell membranes, is an important non-polar component that, due to preferential mixing with sphingolipids, can give rise to lipid rafts as described by the umbrella model. ${ }^{22,23}$ As a result, lipids in a bilayer arrangement display, depending on saturation and length of the acyl chain and the presence of sterols, varying degrees of lateral mobility as dictated by its phase behaviour. ${ }^{24,25}$

Another interesting group of lipids are bolaamphiphiles, also called bolalipids, commonly found in the membrane of thermophilic and acidophilic archeabacteria. Bolalipids are comprising of two hydrophilic head-groups bridged by single or double hydrophobic chains and have a similar shape factor when compared to PC. The unique architecture of these lipids has been suggested to stabilize the archeabacteria membrane under extreme 
conditions. Bolalipids self-assemble into a variety of structures in aqueous environment like spherical and thread-like micelles where they can adopt a U-shape, membrane spanning or a combination of both conformations. Recently, micelles of the bolalipid GLH20 were used to prepare mimetic membranes on silica at high (150 mM) and low (1 $\mathrm{mM}$ ) ionic strength. In addition, GLH-20 containing membranes were modified to house the transmembrane protein aquaporin PM-28. ${ }^{26}$ This concept has been extended to develop bolalipids-based membranes for water filtration. ${ }^{27}$

Phytanoyl lipids can be found in extremophiles, such as archeabacteria, and are phase stable over large temperature ranges, -120 to $120^{\circ} \mathrm{C}$. Their chemical structure is characterized by branched apolar tails, resulting in disordered packing and a high degree of membrane fluidity. ${ }^{28}$

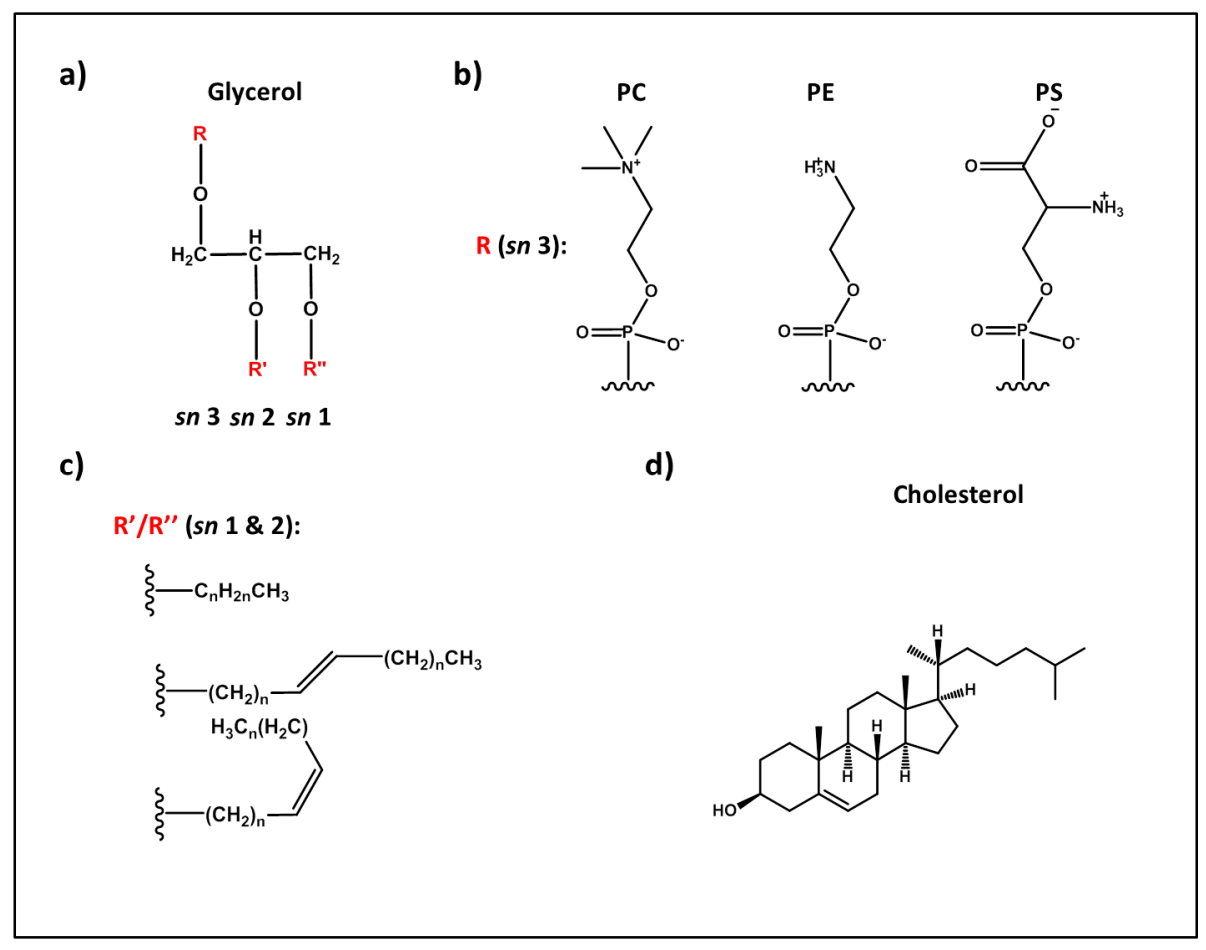

Figure 1.3: Overview of the various lipids; a) the glycerol backbone and the structures of the most common $s n-3$ position derivatives, b) $s n-2$ and $s n-3$ positions can be modified with saturated or unsaturated acyl tails and $\mathbf{c}$ ) the structure of the most common sterol, cholesterol.

Lipopolysaccharides (LPS) have also been used in relation to model membranes. LPS are present in the outer membrane of gram-negative bacteria and are composed of a lipid A, 
an inner core and outer core and a strain specific O-antigen. The O-antigen is involved in pathogenicity and immune response in higher organisms. To study glycan-binding proteins, lectins, LPS have recently been incorporated in PC mimetic membranes. ${ }^{29}$

In addition, a large variety of synthetic lipids have been prepared. For example, fluorescent lipids are sometimes required for biophysical characterization studies. A fluorescent dye can either be introduced to the head-group of ethanolamine lipids or to the acyl tail. These fluorescent lipids are often used to stain the lipid bilayer of cells or model membranes. Other synthetic lipids are available where the head-group is modified with, for example, maleimides or biotin groups. Biotinylated and maleimide-modified lipids are attractive synthetic lipid building blocks for conjugation of any desired functional molecules after which these molecules can be anchored in the membrane.

\subsection{Model membranes}

Given that natural cell membranes are complex multimolecular systems, model membranes consisting of phospholipids present an attractive platform to mimic cell-ECM and cell-cell interactions. Moreover, model phospholipid membranes allow for the investigation of biological processes at the cellular level and for the study of membrane components in their native membranous environment. The first model cell membranes were developed by Mueller and co-workers in $1962 .^{30}$ They developed so-called free standing 'black lipid membranes'. However, only from the mid-eighties of the last century model membranes became more commonplace with the introduction of SLBs. These SLBs were able to capture key features of natural membranes like the lateral mobility of lipids and associated ligands as pioneered by McConnell and co-workers. ${ }^{31,32}$ Two techniques that are most frequently used to form SLBs will be described in the following section. Then, we pay attention to substrates that are used for SLB formation and to what extent patterning can be useful. We briefly touch upon techniques that can be used for analysing SLBs. $^{33-36}$

\subsubsection{SLB formation}

One frequently encountered method to prepare SLBS utilizes LangmuirBlodgett/Langmuir-Schäfer (LB/LS) techniques (Figure 1.4a). Typically in a Langmuir trough a lipid monolayer can be formed on a water surface from an organic solvent. After evaporating the solvent, the surface pressure is increased to the equivalent pressure of the bilayer. A cleaned hydrophilic sample is quickly submerged into the trough and slowly lifted from the solution while maintaining a constant surface pressure. As a result, a lipid monolayer, known as the LB layer, is transferred onto the sample surface. A second monolayer, the LS layer, is spread and compressed on the through in a similar fashion. The LB-coated sample will contact the LS-monolayer at the air/water interface for a few 
seconds. To complete the bilayer formation the sample is pushed through the interface and kept submerged while the supported bilayer sandwich is removed from the trough. ${ }^{37}$

Another main technique to form SLBs uses a solution of unilamellar lipid vesicles or proteoliposomes that are fused with solid supports (Figure 1.4b). Such vesicles can be obtained using standard liposome preparation or protein reconstitution methods i.e. extrusion or sonication. Often a lipid mixture, dissolved in a common azeoptropic organic solvent, is dried under a stream of nitrogen and under reduced pressure. After hydration and vortexing, either sonication or extrusion of multilaminar vesicles (MLVs) yield monodisperse small or large unilaminar vesicles (SUVs or LUVs).

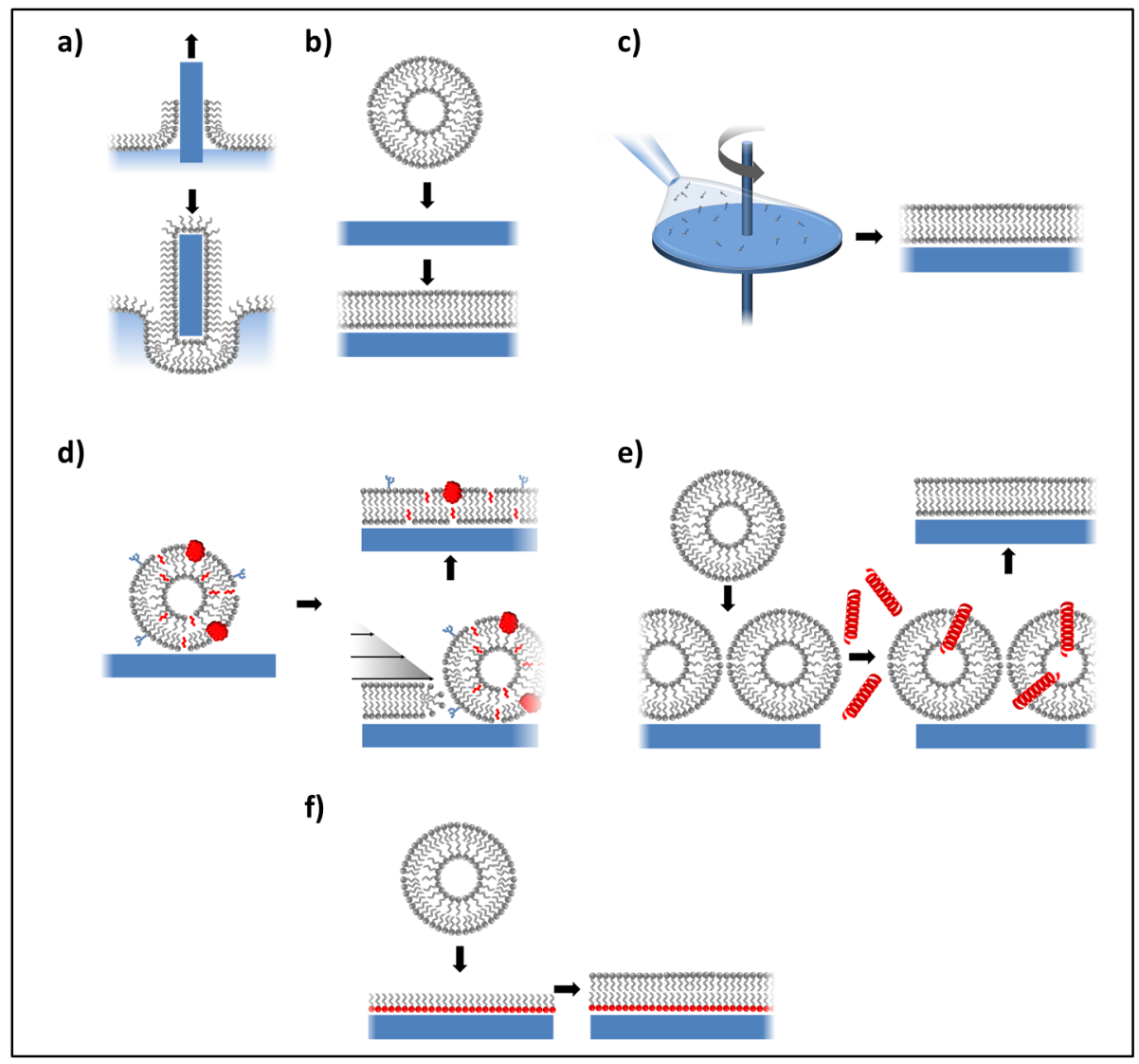

Figure 1.4: Common SLB formation techniques; a) Langmuir-Blodgett/Langmuir-Schäfer, b) vesicle fusion, c) spin-coating, d) hydrodynamically driven vesicle fusion, e) vesicle fusion induced by $\alpha$ helical peptides and f) combination of Langmuir-Blodgett and vesicle fusion. 
Extrusion provides more control over the size distribution as compared to sonication as can be easily monitored by transmission electron microscopy (TEM) and dynamic light scattering (DLS). ${ }^{38}$ Upon incubation with freshly prepared solutions of SUVs or LUVs ${ }^{39}$ with solid supports, such as mica, glass and silica, SLBs are formed when conditions, such as ionic strength and $\mathrm{pH}$, are taken into account. The SLB formation follows two critical steps, i.e. (1) vesicle adsorption onto solid supports and their subsequent rupture and (2) the formation of bilayer patches and their completion to form continuous SLBs. The formation of an SLB using vesicle fusion follows an energetically favourable process. The polar heads of lipids align towards the polar, aqueous environment and the hydrophobic tails tend to cluster together, thus forming the lipid bilayer after vesicle fusion. As a result, water molecules are released from the vesicle giving the surrounding water structure a higher entropy. In addition, hydrophobic effects, intermolecular van der Waals interactions, hydration and electrostatic effects have an impact on the formation and organization of SLBs. After SLB formation, a thin water layer of 6-20 $\AA$, referred to as hydration layer, is found between the proximal leaflet and the solid support. ${ }^{40,41}$ Solid supports with different interfacial properties affect the formation of SLBs. High energy, hydrophilic supports tend to yield SLBs while low energy, hydrophobic ones prompt lipid monolayer formation. Although spontaneous fusion with certain surfaces is reported and comprehensively reviewed by Brisson and co-workers ${ }^{42}$, this fusion process can be problematic in the case of more complex vesicle compositions, such as vesicles with high cholesterol content or when multiple types of lipids are used. Fusion of non-fusogenic vesicles compositions to solid supports can be induced by optimization of experimental conditions, e.g. temperature, $\mathrm{pH}$, osmolality, cation valency and so forth. Fusion of non-fusogenic vesicles has also been induced by the edge of a hydrodynamically driven SLB within a microfluidic device (Figure 1.4d). Using this approach cell membrane-derived SLBs were prepared over large surface areas, while displaying expected lateral mobility. ${ }^{43}$ Also, $\alpha$-helical peptides have been described to facilitate fusion (Figure 1.4e) ${ }^{44}$ and the use of a freeze-thaw cycle has been suggested. ${ }^{45} \mathrm{An}$ in depth overview of the various methodologies to induce fusion of vesicles can be found in a recent review of Zauscher and co-workers. ${ }^{46}$

In addition, Langmuir-Blodgett and vesicle fusion can be combined to form SLBs (Figure 1.4f). The proximal leaflet is then first applied using the Langmuir trough while the distal leaflet is deposited via vesicle fusion in a second step. Since ligand containing vesicles can be introduced at this stage, only the distal leaflet will carry functional groups. ${ }^{47}$ Fast preparation of lipid bilayers can be achieved using spin-coating (Figure 1.4c). Lipids dissolved in an organic solvent are deposited and spin-coated onto cleaned substrates. This techniques allows for preparation of lipid multilayers with control over the amount of lipid bilayers formed. ${ }^{48}$ Formation of single bilayers can be achieved by fine-tuning the 
spin-coating method ${ }^{49}$ which has recently been exploited to form a single bilayer on polymer-grafted supports. ${ }^{50,51}$

\subsubsection{SLB substrates and architectures}

A wide variety of substrates were modified with SLBs on which numerous SLB architectures can be fabricated often designed for specific applications. Figure 1.5 displays selected SLB architectures that are frequently used such as solid, particle, tethered, cushioned, free-standing, double and multi-layered SLBs. Initially, silica-based materials such as glass, mica, $\mathrm{SiO}_{2}$ were used, but nowadays polydimethylsiloxane (PDMS), titania, alumina, PEM-films can be used for SLB formation as well. Gold substrates modified with hydrophilic linkers or amphiphiles have been used for the formation of so-called tethered SLBs as recently was summarized. ${ }^{52,53}$ Formation of SLBs can be achieved on untreated, lipophobic gold surfaces by depositing e.g. disaccharide trehalose. ${ }^{54}$

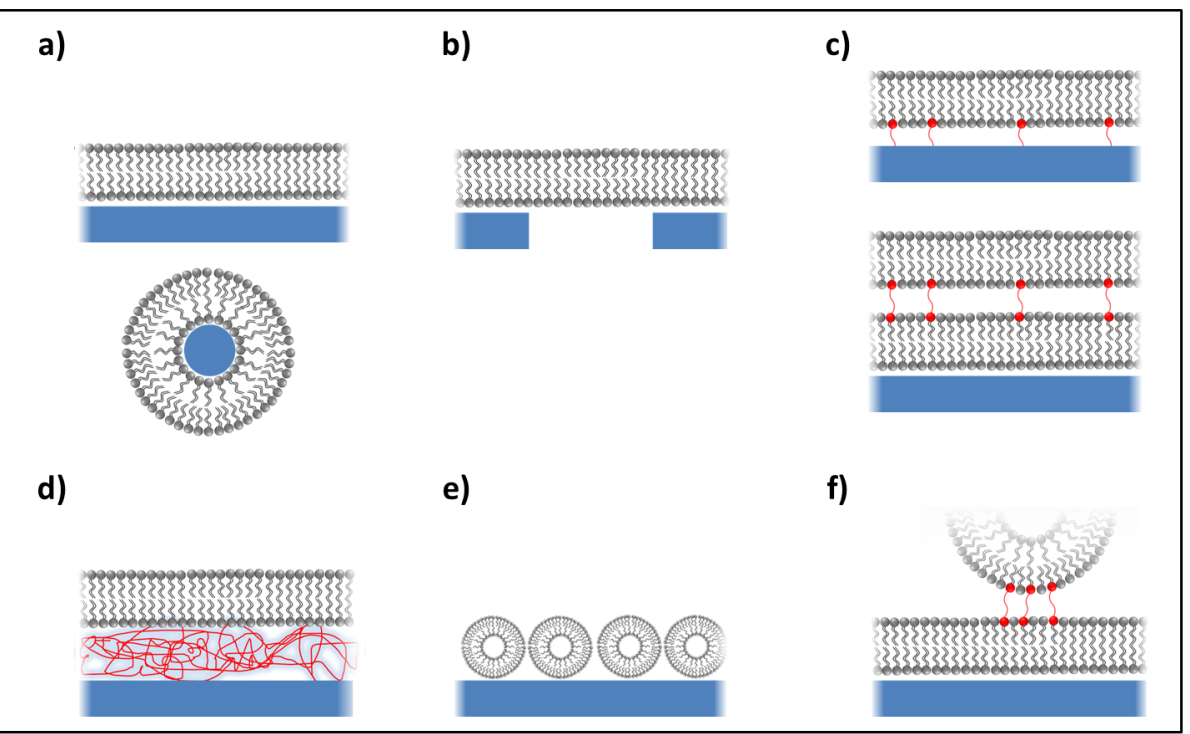

Figure 1.5: Selected SLB architectures; a) solid and particle supported, b) free standing, c) tethered and SLB multilayers, d) cushioned, e) vesicle layer and f) SLB supported vesicle layer.

The resulting glassy layer resulted in proper vesicle fusion and lipid lateral diffusion could be observed. ${ }^{54}$ Successful SLB formation is recently reported on a few biomaterials as well like hydroxyapatite sol-gel or xerogel films ${ }^{55,56}$ and nanofibrous polycaprolactone (PCL) mats coated with $\mathrm{SiO}_{2}{ }^{57}$ 
Although glass or other surface supported SLBs are most frequently used, this type of SLB has typically a limited aqueous volume provided by the hydration layer between the bilayer and the solid support. Cushioned SLBs have been developed to position the SLB further away from the support as reviewed by Tanaka and Sackmann ${ }^{58}$ and more recently by Knoll and co-workers. ${ }^{52,53,59-61}$ Cushioned SLBs are better suited to accommodate large transmembrane proteins since the increased distance above the substrate avoids frictional coupling between the SLB and the substrate when the transmembrane proteins are engaged with other biomolecules. Cremer and co-workers proposed doubly cushioned architectures using PEGylated SLBs and passivated glass supports. They found that this doubly cushioned arrangement preserves the lateral mobility (ca. $\mu \mathrm{m}^{2} / \mathrm{s}$ ) of model protein Annexin $V$ with high mobile fractions (75\%). ${ }^{62}$ In another example, Vancso and co-workers studied the use of charged polymer layers for vesicle fusion. Here, in situ polymer growth using atomic transfer radical polymerization (ATRP) of zwitterionic polySMBA ${ }^{\text {a }}$ was studied. Successful SLB formation was observed with conserved lateral mobility of fluorescently labelled zwitterionic DOPC ${ }^{b}$ lipids. In this case controlled length and grafting density of the brush allows for modulation of the adhesion strength of SLBs to the surface. $^{63}$

\subsubsection{Patterning of SLBs}

A variety of techniques have been used to fabricate patterned SLBs (Figure 1.6). The preparation of surfaces with patterns of SLBs can be achieved e.g. by scratching the tip of a pair of tweezers over the surface to locally remove lipids (Figure 1.6a). Alternatively, a tip of an atomic force microscope (AFM) can be used to locally remove material from a substrate. This so-called nanoshaving lithography was used to remove bovine serum albumin (BSA) from a fully BSA-coated substrate (Figure 1.6h). Back-filling of these empty regions with SLBs has been achieved with dimensions of $50 \mathrm{~nm}$ in width. ${ }^{64}$ However, healing of the scratches through SLB spreading was observed limiting the lifetime of SLB patterns. In general, both negatively and positively charged SLBs tend to spread at $\mathrm{pH}$ values below ca. 10-11 depending on the ionic strength of the buffer. When membrane components are first separated by an electric field, scratching can be used to permanently partition these components at $\mathrm{pH} 10.3$ to prevent healing of the scratch. ${ }^{65}$

Modification of the underlying substrate with physical barriers prior to bilayer formation allows for the formation of well-defined permanent SLB patterns (Figure 1.6b). This was shown initially by Boxer and co-workers who made use of photolithography to prepare patterns of photoresist, aluminum oxide, or gold on oxidized silicon substrates. After vesicle fusion, the SLB was partitioned into micrometer-sized arrays, often referred to as

\footnotetext{
a PolySMBA: poly(sulfobetaine methacrylate)

${ }^{b}$ DOPC: 1,2-dioleoyl-sn-glycero-3-phosphocholine
} 
corrals, ${ }^{66}$ across which no lipid mixing occurs. ${ }^{67}$ Depending on the material used to prepare the barriers, vesicle fusion can or cannot occur on these surfaces. For example, aluminum oxide prevents vesicle fusion while vesicles will adsorb onto chromium, gold and indium tin oxide, albeit with no or limited lateral mobility.

Micro-contact printing using hydrophilic PDMS stamps can be used to selectively deposit or remove SLB patches to yield patterned SLBs (Figure 1.6c). In addition, direct microcontact printing of e.g. BSA proteins prohibits formation of an SLB in these regions also resulting in a patterned SLB surface. ${ }^{68}$

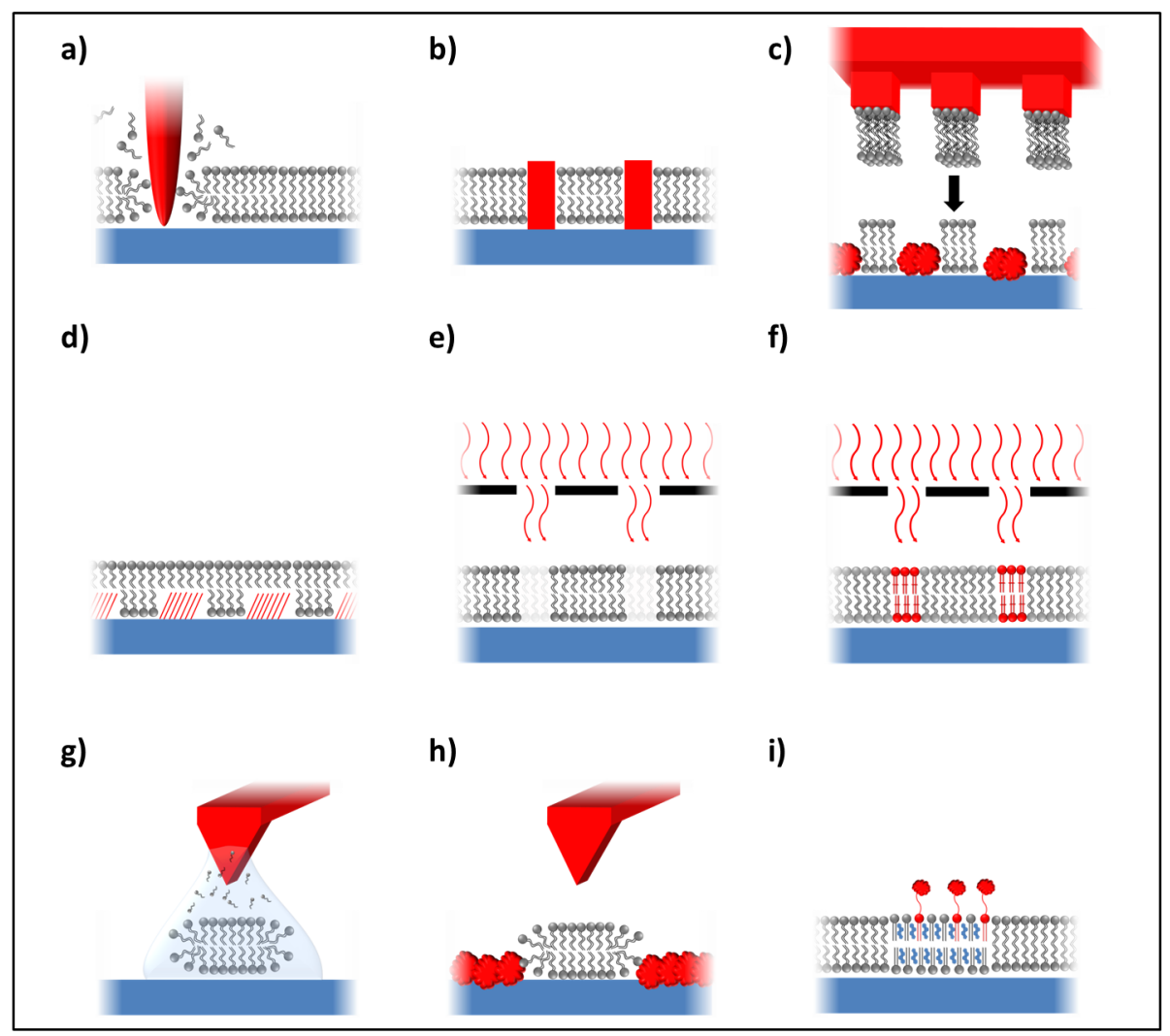

Figure 1.6: Selected SLB patterning methods; a) scratching, b) physical barriers, c) hydrophilichydrophobic patterning, d) micro-contact printing, e) direct UV patterning, f) preparation of photocross-linked SLB barriers, g) dip-pen nanolithography, h) nanoshaving and i) in situ patterning by domain targeting. 
In these cases the protein layers form barriers across which no immediate lipid mobility was observed. This method can also be used to embed additional functionality to the SLB patterned surfaces when the ECM protein fibronectin was deposited instead of inert BSA proteins. ${ }^{69}$ More recently, poly-L-lysine-grafted-PEG (PLL-PEG) was used for creating surface patterns by micro-contact printing. The non-printed regions were subsequently modified with an SLB. These PLL-PEG patterns confined the lateral mobility in the SLB and were shown to be stable up to several weeks, which is difficult to achieve using protein barriers. ${ }^{70}$ Alternatively, patterned SLBs can be achieved by a technique referred to as micro-contact displacement that makes use of a patterned PDMS stamp with addressable reservoirs that is placed over an SLB. When the PDMS stamp contacts the SLB substrate lateral mobility is confined to the areas of the stamp that are not in contact with the substrate. Confinement was confirmed upon injection of a fluorescent dye into the addressable reservoirs. $^{71}$

Direct printing of vesicle solution is another possibility to fabricate patterned SLBs. In this case stamps were prepared from agarose instead of PDMS. Here, agarose stamps with a pore size of ca. $470 \mathrm{~nm}$ were fabricated and each of the posts were loaded with a submicroliter volume of vesicle solution. After stamping on glass slides, mobile and functional SLBs could be formed. ${ }^{72}$ Locally controlling surface wettability has proven successful in preparing patterned mono- and bilayers (Figure 1.6d). In an early example micrometersized hydrophilic regions were prepared using standard photolithography techniques. The hydrophilic squares measuring from $25 \times 25 \mu \mathrm{m}$ to $250 \times 250 \mu \mathrm{m}$ in size were individually addressed using a capillary containing lipid vesicles. This process enabled the preparation of discrete SLBs with unique compositions. ${ }^{73}$ More recently discrete SLBs have been achieved by micro-contact printing of hexadecanethiol and back-filling with a glycolterminated thiol on gold substrates. These patterned hydrophobic and hydrophillic surfaces were used to fabricate surfaces with areas with lipid mono- or bilayers, respectively. ${ }^{74}$ Similarly, octadecyltrichlorosilane (OTS) modified $\mathrm{SiO}_{2}$ surfaces can be patterned by UV illumination through a photo-mask yielding hydrophobic and hydrophilic domains suitable for lipid mono- and bilayer formation, respectively. ${ }^{75}$ In another example, a transmission electron microscopy grid was placed on a flat PDMS surface serving as a mask. After plasma oxidation the exposed areas become hydrophilic while the covered areas retain their hydrophobic nature. Subsequent addition of lipid vesicles yields lipid mono- and bilayers. ${ }^{76}$

A very straightforward method of preparing patterned SLBs is the use of short-wavelength UV irradiation to locally induce photodecomposition of lipids (Figure 1.6e). An UV mask is placed just above the SLB while submerged in buffer. Exposure of lipids to UV leads to their removal from the SLB and can be used for back-filling with an alternate vesicle 
solution. Depending on the lateral mobility of the lipids, this process yields stable or unstable patterns. $^{77}$

Apart from site-selective removal of lipids, UV illumination can be used to crosslink Diynelipids that bear diacetylene bonds in their apolar tails (Figure 1.6f). In a recent example, DiynePC and DiynePE-based SLBs were prepared on glass supports via the LangmuirBlodgett/Langmuir-Schäefer method and using a UV-mask site-selective crosslinking was achieved when applying a grid during illumination. These polymerized SLB patches restrict the lateral mobility and display a high degree of stability. Unreacted Diyne-lipids can be removed using a detergent solution while leaving the cross-linked grid unaffected. Subsequent incubation with lipid vesicles results in fusion in the exposed regions. Grafting cell adhesive peptides to the ethanolamine head-group of DiynePE and backfilling with a non-fouling DOPC-SLB, resulted in selective adhesion of $\mathrm{NIH}_{3} \mathrm{T3}^{\mathrm{C}}$ fibroblasts to those surfaces. Grafting biotin to the head-group of DiynePE and the surface of a PDMS microwell stamp, allowed for direct bonding of the PDMS to the cross-linked SLB by means of biotin-streptavidin interaction. ${ }^{78}$

The use of microfluidics is another tool to achieve patterned SLBs. Alternatively microfluidic devices can enable bimolecular interaction studies to the SLBs. Simple capillary action of fluid containing vesicles enabled the formation of discrete SLBs. ${ }^{79}$ The size of the SLB patterns is determined by the size of the PDMS flow channels. Cremer and co-workers developed a multi-microchannel PDMS device for the study of binding events at SLB interfaces, which were deposited on the substrate and PDMS walls of the device. ${ }^{80}$ For example, antibody binding to target epitopes, which were presented on the SLBs, ${ }^{81,82}$ and $\mathrm{Cu}$ (II) binding to PS lipids ${ }^{83}$ was scrutinized in a multiplexing fashion enabled by such a microfluidic device. ${ }^{84}$ As a continuation, protein patterns were prepared inside the PDMS flow channel. This resulted in patterned SLBs within the flow device. ${ }^{85}$ Similarly, Kam and Boxer showed the use of a patterned substrate onto which a PDMS flow channel was assembled. Introducing simultaneously two liquid flows each containing vesicles, patterns of SLBs of varying compositions were prepared directly adjacent to each other at the fluid interface of the two streams. ${ }^{86}$

Site-selective lipid bilayer formation can be achieved using dip-pen nanolithograpy (DPN) (Figure 1.6g). The scanning probe-based method uses the tip of the cantilever to transfer an inking solution to the substrate. Including lipids in the inking solution enabled Fuchs and coworkers to generate patterns of SLBs that in turn could be used to pattern proteins. ${ }^{87,88}$ Further fine-tuning of the experimental conditions like humidity and scan

\footnotetext{
${ }^{c}$ NIH3T3: fibroblast cell line 
speed, single lipid bilayer deposition can be ensured with sub-100 $\mathrm{nm}$ lateral resolution. ${ }^{88}$ Micrometer-sized SLB spots, can be prepared by non-contact dispensing of lipid vesicles to a substrate through a thin layer of water. Using this approach spots of SLBs with sizes varying from 180 to $360 \mu \mathrm{m}$ in diameter were fabricated. This simplified method does not require complex buffer formulation, humidity control and avoids contamination and disruptive drying of the SLB. ${ }^{89}$

In situ generation of ligand patterns in SLBs has been shown (Figure 1.6i) by e.g. applying standing acoustic waves. ${ }^{90}$ This highly dynamic, self-organizing method for ligand patterning uses piezoelectric lithium tantalite substrates and metallic interdigital transducers. When a periodic alternating voltage is applied, a periodic deformation and propagation of waves is observed. Standing waves can be generated by the superposition of two waves propagating in opposite direction. These standing 'shear' surface acoustic waves are transferred to the lipid bilayer, inducing variations in the density of the membrane, resulting in transport and accumulation of labelled lipids. In this study, headgroup modified lipids containing biotin were used to load the bilayer with streptavidin and/or avidin. The patterns that were generated with the device were reversible under low surface coverage of biotin-anchors $<10 \%$. After the device is turned off the native environment was restored. ${ }^{90}$

Surface topography has been adjusted to study SLB dynamics. ${ }^{91}$ For instance, the preparation of SLBs on regularly curved glass substrates affected the phase behaviour and was shown to perturb protein sorting in the cell membrane of cells that were cultured on such an SLB platform. ${ }^{92}$ Complementary information regarding SLB nanoscale complexity can be found elsewhere. ${ }^{93}$

\subsection{SLB analysis}

Throughout the years a wide range of surface sensitive techniques have been used to study and characterize SLBs (Figure 1.7). Fluorescence analysis is particularly widely used to visualize membrane-bound components and to gain insights into the lateral mobility of lipids in the membrane (Figure 1.7a). Upon incorporation of a lipid-dye conjugate in the SLB, a so-called fluorescence recovery after photobleaching (FRAP) experiment can be conducted. In a FRAP experiment a portion of the fluorophores are photobleached resulting in a dark region in the SLB. Since liquid-state SLBs display lateral mobility, unbleached lipid-dye conjugates will diffuse into the bleached region, while the bleached molecules diffuse out. Monitoring this region in time and accounting for acquisition bleaching, a fluorescent recovery curve can be compiled. This curve provides information about (1) the mobile fraction and (2) can be used to estimate a diffusion coefficient $(D)$ of the lipids in the SLB. Calculation of the $D$-value depends on the shape 


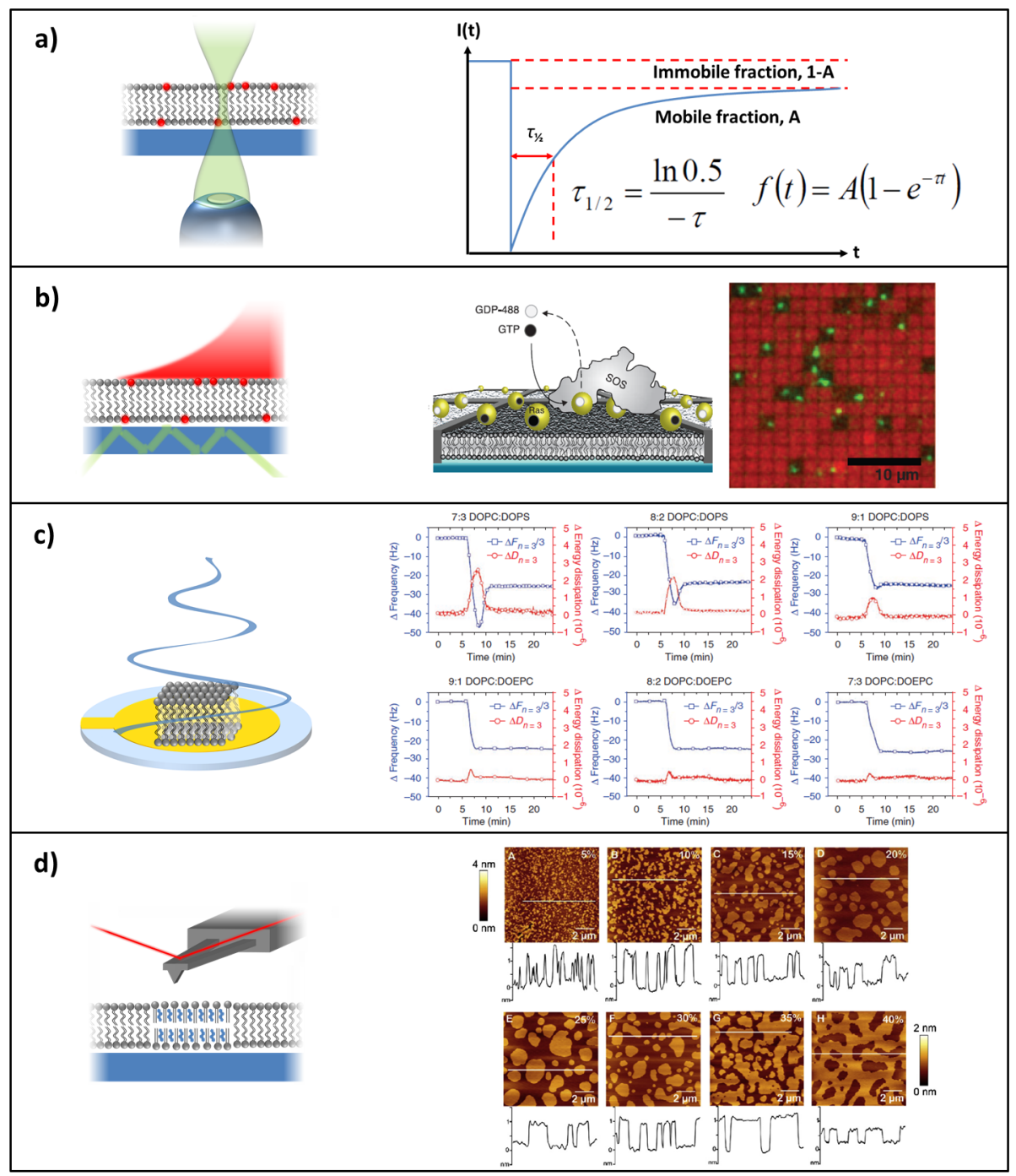

Figure 1.7: Examples of techniques to analyse SLBs; a) FRAP analysis provides information of the lateral mobility and mobile fraction of lipids in SLBs. b) TIRF microscopy enables fluorescent analysis in close proximity of the glass interface as was shown recently by Groves and co-workers. Reprinted with permission. ${ }^{94}$ Copyright 2014, American Association for the Advancement of Science. The exclusion of bulk effects enabled the study of single molecules associated with the SLB. c) QCM-D provides insight into SLB formation, visco-elastic properties and binding events. Examples of vesicle fusion, as studied with QCM-D, are given for different lipid compositions. Reprinted with permission. ${ }^{95}$ Copyright 2010, Nature Publishing Group. d) AFM in liquid provides insights into SLB topography and allows identification of liquid-ordered, raft domains, in DOPC, SM SLBs with varying amounts of cholesterol. Reprinted with permission. ${ }^{96}$ Copyright 2010, Cell Press. 
and profile of the bleached region. The Axelrod approximation is used frequently to gain insights into the $D$-value for a Gaussian spot, because of its ease-of-use. ${ }^{97}$ In recent years, freeware software ${ }^{d}$ to analyse FRAP data has become available that allows for more complex fitting of the experimental data to Bessel functions as described by Soumpasis et al. for diffusion-dominated recovery in circular bleach spots. ${ }^{98}$ However, these methods are prone to error, namely temporal and spatial variations in intensity due to sample drift, acquisition bleaching or uneven illumination of the image jeopardize correct data treatment. Kapitza et al. proposed a method where the intensity of only the central part of the bleached spot is taken to deduce the $D$-value. ${ }^{99}$ Over time, improvements have been made to more accurately deduce the lateral mobility of lipids in SLBs. For example, Höök and co-workers proposed a method ${ }^{\mathrm{e}}$ to overcome shortcomings of the aforementioned approaches. ${ }^{100}$ Their method is based on circular averaging of each image, and spatial frequency analysis of the averaged radial data that requires no prior knowledge of the shape of the bleached area. Fluorescence analysis can be used to image domains within the lipid bilayer, as reviewed by Crane and Tamm. ${ }^{101}$

Other commonly applied fluorescent microscopy techniques to analyse and study SLBs are total internal reflection fluorescence (TIRF) microscopy and fluorescence correlation spectroscopy (FCS). TIRF microscopy makes use of the evanescent wave that is exclusively generated when total internal reflection of the incident light occurs at the glass-water interface. Since the evanescent field decays exponentially, this technique allows for fluorescent analysis $<100 \mathrm{~nm}$ from the glass interface, thus minimizing bulk effects. ${ }^{102}$ Very recently, a TIRF study was reported on the single-molecule activity of SOS ${ }^{f}$-catalysed nucleotide exchange at the SLB interface by Groves and co-workers (Figure 1.7b). ${ }^{94}$ Correlation analysis of the fluctuation of the fluorescence intensity as determined from FCS data can be used to determine the concentration and lateral diffusion of dye-labelled lipids and proteins with a high statistical accuracy. Such data can be used to identify hindered diffusion as reviewed by Macháň and Hof. ${ }^{103}$

Insights into physical properties of SLBs without the need for labelling of the lipids can be gained using acoustical and optical-based sensing methods. For example, SLBs have been studied with surface plasmon resonance (SPR) to elucidate surface interactions, ${ }^{104}$ ellipsometry for measuring layer thicknesses, ${ }^{105}$ reflectometry to probe the effective thickness and refractive index ${ }^{106}$ and quartz crystal microbalance with dissipation monitoring (QCM-D) to measure adsorbed mass and visco-elastic properties. ${ }^{95}$ Acoustical sensing with QCM-D in particular helped to elucidate the process of vesicle fusion on various supports and different conditions (Figure 1.7c). ${ }^{42,107}$ Moreover, QCM-D can be

\footnotetext{
${ }^{\mathrm{d}}$ FRAP analyser: available from the University of Luxemborg

e Matlab plugin: available from Magnus Jonsson, Linköping University

${ }^{f}$ SOS: son of sevenless
} 
combined with complementary optical techniques such as ellipsometry. ${ }^{108}$ The use of QCM-D has recently been expanded to study bone mesenchymal stem cell adhesion on SLBs with or without cell adhesive peptides. Comparing the $\Delta D / \Delta f$ plots for the various surfaces, differences in adhesion density and properties could be identified. ${ }^{109}$ Electrical analysis of SLBs with or without embedded membrane proteins has been shown in particular with tethered SLB architectures, which is reviewed by Knoll and coworkers. $52,53,61$

Scanning probe techniques such as AFM can be used to measure the thickness of SLBs ${ }^{110-}$ 112 and the rigidity of pore spanning SLBs and to map the surface topography to, for example, visualize lipid rafts (Figure $1.7 \mathrm{~d}$ ). ${ }^{96,113}$ The use of AFM in studying phase transitions in SLBs have recently been reviewed. ${ }^{114}$ Combinations of QCM-D and AFM have been adopted to explore the intermediate states leading to SLB formation. ${ }^{107}$ Also, AFM and TIRF microscopy has been successfully applied together to distinguish between lipid domains. $^{115}$

Small angle neutron scattering (SANS), nuclear magnetic resonance (NMR), Fourier transform infrared spectroscopy (FTIR) can be used to probe structural properties and chemical composition of SLBs. ${ }^{58,116}$ Boxer and co-workers used secondary ion mass spectrometry (SIMS) to identify the size of cholesterol-dependent phases of monosailo ganglioside (GM1) in a canonical lipid raft composition of lipids. ${ }^{117}$ Matrix-assisted laser desorption/ionization (MALDI) mass spectroscopy was shown on frozen SLB samples of which the components were separated by an electric field. ${ }^{118}$

\subsection{SLB characteristics}

\subsubsection{Lipid phase behaviour and mobility}

A unique feature of lipid bilayers is their lateral fluidity and its central role in an assortment of biological functions e.g. signalling, transport and intermembrane interactions. The chemical make-up of, predominantly, the acyl tails determines lipid phase behaviour and segregation in lipid bilayers. Various temperature-dependant states can be distinguished in which lipids can reside while adopting a bilayer arrangement e.g. liquid-disordered $\left(L_{\alpha}\right)$, liquid-ordered $\left(L_{o}\right)$ and solid (gel, $\left.L_{\beta}\right)$ phases (Figure 1.8a). The $L_{\alpha}$ phase is characterized by a disordered packing of lipids due to the presence of unsaturated acyl chains. The $L_{\alpha}$ phase yields a high lateral mobility in the $\mu \mathrm{m}^{2} / \mathrm{s}$ range and a low $T_{m}$ (melting temperature). Lipids bearing saturated acyl chains tend to pack more tightly, increasing their $T_{m}$ and resulting in a reduced later mobility of less than $10^{-3} \mu \mathrm{m}^{2} / \mathrm{s}$. As described previously, sterol-dependant raft states, also referred to as $L_{0}$, demonstrate a high degree of packing while retaining a high mobility of $\mu \mathrm{m}^{2} / \mathrm{s}$. Observation of lipid rafts 
in SLBs can be achieved using e.g. scanning probe techniques (vide supra). ${ }^{119}$ Membrane fluidity is often the result of (1) physical properties of constituent lipids and (2) sterol concentration. It must be noted that the $T_{m}$ of a lipid bilayer, the temperature at which long range lateral mobility is observed, does deviate from the temperature at which mobility is observed in lipid monolayers of similar lipid composition. ${ }^{120}$ In addition, there is evidence that, in the case of SLBs, the substrate can affect the diffusion behaviour of the proximal leaflet. For example, it has been found that on mica, the lateral diffusion of the proximal leaflet is slower as compared to the one observed in the distal leaflet, even though the $T_{m}$ was found to be the same. ${ }^{121}$ Apart from the lateral diffusion of lipids, angular rotation of lipids around their axis perpendicular to the plane can occur.

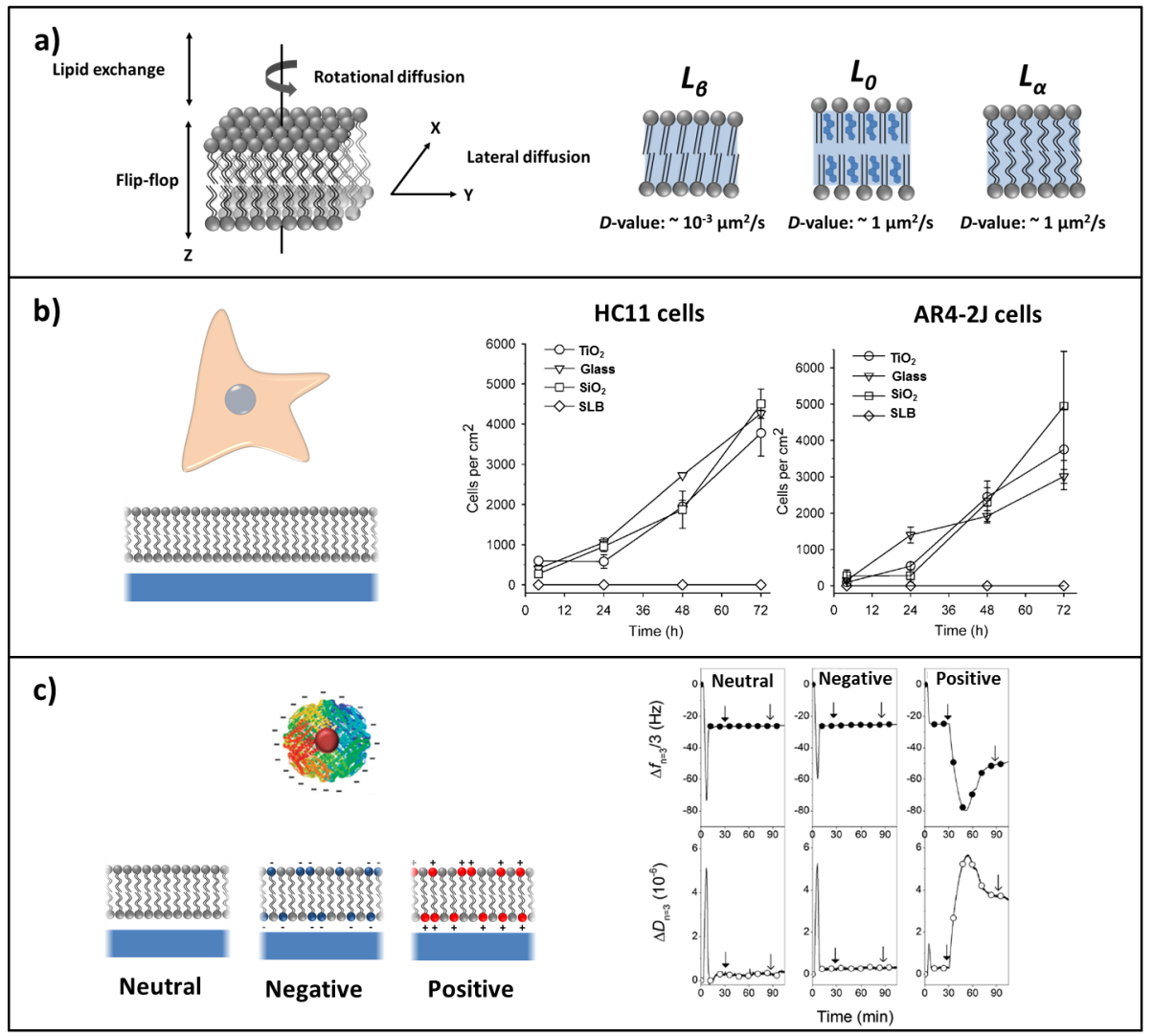

Figure 1.8: SLB characteristics; a) lipid dynamicity in SLBs, b) cell adhesion on zwitterionic, liquidstate SLBs. Reprinted with permission. ${ }^{116}$ Copyright 2003, John Wiley \& Sons. c) Protein absorption on zwitterionic and charged liquid-state SLBs as determined with QCM-D. Reprinted with permission. ${ }^{122}$ Copyright 2012, Royal Society of Chemistry. 
Lipid phase separation behaviour in binary lipid mixtures, which are used for the preparation of SLBs, can be exploited for in situ patterning. This was shown for DSPC ${ }^{\mathrm{g}}$ - and DOPC-based SLBs that were doped with nickel chelating DOGS ${ }^{\text {h}}$-lipids for the immobilization of His-tagged proteins. ${ }^{123}$ In both DPSC- and DOPC-based SLBs, patterns of a His-tagged green fluorescent protein (GFP) were generated that correspond to the phase separation pattern of DOGS-nitrilotriacetic acid (NTA). This effect was observed at doping densities of $>10 \%$ of DOGS-NTA. ${ }^{123}$

Patterning could also be achieved when a ternary lipid mixture is used that is able to form liquid-ordered, raft, domains. These rafts can be targeted using proteins carrying a myristoyl and palmitoyl chain. Prenylated proteins display low affinity to raft domains due to their branched tails, ${ }^{124,125}$ while palmitoylation on the other hand is known to yield raftophilic proteins. ${ }^{14}$ That raises the question if tandem modification i.e. prenylation and palmitoylation allows for the modulation of overall raft affinity and targeting in the bilayer. Ligands can be directed to specific areas of sterol-containing ternary lipid bilayers to yield heterogeneous, patterned surfaces by fine-tuning the use of lipid anchors. To our understanding, this remains unexplored in SLB applications.

\subsubsection{Non-fouling nature of SLBs}

Another useful property of SLBs is their inherent non-fouling nature. This non-fouling property is dependent on the composition of the SLB and is particularly strong when zwitterionic PCs are used. QCM-D measurements showed that human fibrinogen, human serum albumin (HAS), bovine haemoglobin and various other naturally occurring proteins, barely adsorbed onto SLBs made of zwitterionic PC. ${ }^{126}$ This behaviour is often ascribed to the neutrally charged $\mathrm{PC}$ head-group in a large $\mathrm{pH}$-range, $3<\mathrm{pH}<10$. In addition, $\mathrm{PC}$ is known to attract a water layer that hampers the adsorption of proteins. The high lateral mobility of PC lipids further favours this protein repellent property. ${ }^{126}$ This phenomenon strongly influences cell adhesion kinetics and is able to prevent cells from adhering to its surface. This was demonstrated early on by culturing $\mathrm{HC} 11^{i}$ and $\mathrm{AR} 4-2 \mathrm{~J}^{\mathrm{j}}$ cells in serum rich conditions on bare egg yoke PC derived SLBs (Figure 1.8b). Compared to glass, $\mathrm{TiO}_{2}$ and $\mathrm{SiO}_{2}$, negligible amounts of cells adhered to the SLB during a 72 hour culture period. QCM$D$ experiments confirmed that the absence of cell adhesion was related to the absence of deposition of serum proteins on the SLB surface. ${ }^{116}$

\footnotetext{
${ }^{g}$ DSPC: 1,2-distearoyl-sn-glycero-3-phosphocholine

${ }^{\mathrm{h}}$ DOGS: 1,2-dioleoyl-sn-glycero-3-succinate

'HC11: mouse mammary gland cells

${ }^{j}$ AR4-2J: rat pancreatoma cells 
The out-of-plan or z-axis mobility of complete zwitterionic SLBs have been implicated to further enhance their non-fouling behaviour. This was shown for patterned lipid monoand bilayers consisting of POPC. Here, preferential ARPE- $19^{k}$ cell adhesion was noted on the lipid monolayers. Lipid composition and lateral mobility were identical for both conditions and fall short of accounting for the observed differences. ${ }^{127}$ Since lipid bilayers possess a hydration layer, which is absent in lipid monolayers, undulatory motions are expected to be less prominent in the latter case. This was studied by incubation of silica particles of $5 \mu \mathrm{m}$ in diameter with similarly patterned surfaces consisting of DMPC lipid mono- and bilayers patches. It was observed that silica particles became fluorescent above the $T_{m}$ due to wrapping of the lipid bilayer around the particle. This effect was not observed for lipid monolayers or below the $T_{m}$ indicative of out-of-plane mobility of liquidstate SLB. $^{128}$

The non-fouling character of zwitterionic liquid-state SLB was recently exploited for capturing of $\mathrm{CTC}^{\prime}$ cells. ${ }^{129}$ CTC can provide information regarding diagnosis, prognosis and treatment of cancers. Due to their low cell number, $<1$ CTC per $10^{9}$ blood cells, enrichment processes have been developed. However, current surfaces fail to suppress the nonspecific interactions and, as a result, affect the purity of isolated CTCs. For example, on such surfaces ca. 5-15 cells $/ \mathrm{mm}^{2}$ bind non-specifically when incubated with cells at densities of 10,000 , which is still 10 times lower than pure blood. ${ }^{130}$ Using a biotinylatedSLB decorated with anti-EpCAM antibodies directed against the overexpressed epithelial cell adhesion molecule in cancer cells, over $97 \%$ and $72 \%$ of blood spiked with cancer cell lines $\mathrm{HCT} 116^{\mathrm{m}}$ and colo205 ${ }^{\mathrm{n}}$ were retrieved, respectively. Moreover, blood cells could be washed away with ease resulting in CTC purities exceeding 95\%. In addition, cell expansion was shown on the modified SLB surfaces. Strikingly, only one of the two anti-EpCAM antibodies used for cell capture was able to support long-term cell culture on tissue culture plastic even though their affinity was similar. ${ }^{129}$

Although non-fouling characteristics have often been described for liquid-state SLB, zwitterionic gel-state SLBs do however retain this feature to some extent. QCM-D analysis of MPPC-based gel-state SLBs in the presence of serum showed negligible protein fouling on these surfaces. ${ }^{131}$ In addition, a cell adhesion study performed on polymerized zwitterionic SLBs consisting of Bis-SorbPC showed a high degree of cell repellence towards murine derived macrophages. ${ }^{132}$ In addition, cross-linked lipid bilayers have also been

\footnotetext{
${ }^{k}$ ARPE-19: human retinal pigment epithelium (RPE) cell line with normal karyology

' CTC: circulating tumour cells

${ }^{m}$ HCT116: colorectal cancer cells, RAS mutation in codon 13

${ }^{\mathrm{n}}$ Colo205: human Caucasian colon adenocarcinoma cells
} 
investigated for use as a coating in glass capillaries to prevent protein fouling during capillary zone electrophoresis (CZE) ${ }^{133}$ measurements. ${ }^{134}$

In contrast to the non-fouling properties of neutrally charged lipids, protein adsorption can be stimulated by introducing a net charge in the SLB. Indeed, a more in depth investigation in silico revealed that protein adsorption can occur on charged SLBs. From the calculations the adsorption energy between pairs of oppositely charged lipids and charged amino acids was estimated to be $15-20 \mathrm{~kJ} / \mathrm{mol}$. As a protein adsorbs onto the SLB and approaches the Debye screening length, polarization occurs thereby attracting charged lipids. The formation of one lipid-amino acid electrostatic bond yields only short life-times due to the continuous lateral diffusion of charged lipids in SLBs. When 4-5 pairs of lipid-amino acid electrostatic bonds are formed the life-time is increased which may even result in irreversible adsorption. ${ }^{135}$

This was demonstrated using Ferritin, a large iron-storage protein, ${ }^{136}$ as a model protein on SLBs bearing different charges. Previously it was shown that the adsorption of ferritin on charged surfaces was primarily driven by electrostatic interactions (Figure 1.8c). ${ }^{137-139}$ To gain insights into adsorption of the horse spleen ferritin (HSF) to SLBs, QCM-D, SPR and FRAP measurements were used to confirm that protein adsorption is mediated by electrostatic forces, which could be influenced by the $\mathrm{pl}$ of the protein, $\mathrm{pH}$ and charge of lipids. ${ }^{122}$ Since the zwitterionic SLBs show negligible amounts of protein fouling, these SLBs can be explored to study cell-ligand interactions upon modification of the SLB with biologically active ligands (vide infra).

\subsubsection{Air instability of SLBs}

A limiting factor in the use of SLBs is their inherent instability when exposed to air. Any passage through the air-water interface immediately results in delamination of the SLB. ${ }^{33}$ To this end, a variety of methods have been described to stabilize SLBs and prevent delamination (Figure 1.9). To prevent the loss of the structural integrity of SLBs upon exposure to air, SLBs can be post-modified or the support can be modified prior to SLB formation.

Stable SLBs can be observed by chemically crosslinking synthetic lipids, thereby creating polymerized SLBs (Figure 1.9a). For instance, this has been shown for diacetylenic and dienoyl lipids. ${ }^{140-144}$ As described in a previous section, such SLBs lose the lateral mobility of the lipids, however depending on the extent of crosslinking, the use of photocrosslinkable lipids can result in, after back-filling with a liquid-state SLB, tuning of the diffusion coefficient between $0-1.6 \mathrm{\mu m}^{2} / \mathrm{s}$ for Texas red-DHPE ${ }^{\circ}{ }^{145}$ The air stability of

\footnotetext{
${ }^{\circ}$ Texas red-DHPE: Texas red 1,2-dihexadecanoyl-sn-glycero-3-phosphoethanolamine 
these SLBs was not evaluated, but an improved stability is expected depending on the extent of crosslinking.

Post-modification of SLBs with proteins is effective in preventing lipid reorganization upon dehydration. This was shown for biotinylated SLBs, containing 5 mol\% of biotin-PE, coated with streptavidin (Figure 1.9b). ${ }^{146}$ When these streptavidin-SLBs were dried using nitrogen, no mobility of the lipids was observed. However, after rehydration of these SLBs in an aqueous solution the lateral mobility was restored. Lowering of the doping density of biotin-PE caused severe damage to the SLB indicating that the density of the protein layer is important. Other proteins like immunoglobin $\mathrm{G}(\operatorname{IgG})$ showed similar effects. It has been suggested that the presence of a protein layer might stiffen the bending elastic modules of the bilayer thus increasing the energy barrier for delamination, prevent reorganization of the lipids and provide a thin water layer that surrounds the proteins. ${ }^{146}$ Instead of using proteins, the addition of PEG-PE lipids showed an improved stability when exposed to air depending on the PEG chain length and the doping density of the lipid-conjugate. ${ }^{147}$

Another approach to achieve air-stable SLBs is the use of trehalose. This disaccharide sugar vitrifies under drying conditions both intra- and extracellularly. This disaccharide enables anhydrobiotic organisms to survive extreme dehydration for prolonged periods of time. ${ }^{148,149}$ It is postulated that the shell conformation of trehalose can interact with the lipid head-groups and thereby serve as a protective layer on top of the SLB against dehydration upon drying. (Figure 1.9c). ${ }^{150}$ SLBs treated with trehalose can therefore be stored for a longer time. Similar results were achieved in the case of supported lipid monolayers. Recently, trehalose has been used to prepare storable and triggered substrate-mediated vesicle fusion. ${ }^{151}$ Addition of a synthetic trehalose glycolipid provided air stability at doping densities as low as $20 \%$ in the case of supported lipid monolayers. ${ }^{152}$

S-layers, which are crystalline layers of proteins found on the cell surface of archaebacteria, have been shown to stabilize lipid bilayers as well. ${ }^{60,153}$ Introducing Slayers in various architectures on SLBs can stabilize them by mimicking the supramolecular building principles of the bacterial cell membrane. The use of S-layers has recently been summarized by Schuster and Sleytr. ${ }^{154}$

In an alternative approach to achieve air stability, substrates were modified prior to the formation of the SLB with cholesteryl derivatives onto which an SLB was formed stably. Above a certain surface density of cholesteryl groups, FRAP measurements showed no deviation in mobility or mobile fraction after multiple dehydration cycles. ${ }^{155}$ Presumably, surface attached cholesterol groups provide a sufficiently large energy barrier to prevent restructuring of SLBs upon dehydration analogous to the stabilizing effect of cholesterol 
on cellular membranes (Figure 1.9d). This method contrasts with the post-modification methods described above, since repeated dehydration is possible without loss of SLB integrity and fluidity, while maintaining an unmodified SLB surface.

Recently, researchers have succeeded to increase the air-stability by means of physical confinement. ${ }^{156}$ The surface features that enabled air stability were formed by the enzyme phospholipase $A_{2}\left(P L A_{2}\right)$. It was shown that upon hydrolysis of phospholipids by $P L A_{2}$ in a multicomponent SLB, crystal-like structures were formed of $10-20 \mathrm{~nm}$ in height. ${ }^{157} \mathrm{Using}$ a

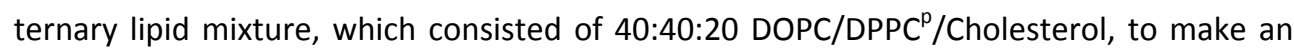
SLB and subsequent incubation with $\mathrm{PLA}_{2}$, resulted in the formation of crystal-like structures. Removal of $\mathrm{PLA}_{2}$ and lipids by washing with detergents revealed a stable network of crystal-like structures that can be used as an substrate for SLB formation. Dehydration and rehydration of a back-filled SLB did not significantly affect its lateral mobility or mobile fraction $\left(1.27 \pm 0.27 \mu \mathrm{m}^{2} / \mathrm{s}, 95 \%\right.$ vs. $1.14 \pm 0.34 \mu \mathrm{m}^{2} / \mathrm{s}, 95 \%$ respectively.). The increased air stability of these SLBs is thought to be caused by the physical confinement imposed by the crystal structures, which reduces interfacial force in the peel-off direction by cutting the water interface. As a consequence, residual water can evaporate with no considerable interfacial force, leaving the SLB unharmed. ${ }^{156}$

${ }^{\mathrm{p}}$ DPPC: 1,2-dipalmitoyl-sn-glycero-3-phosphocholine 


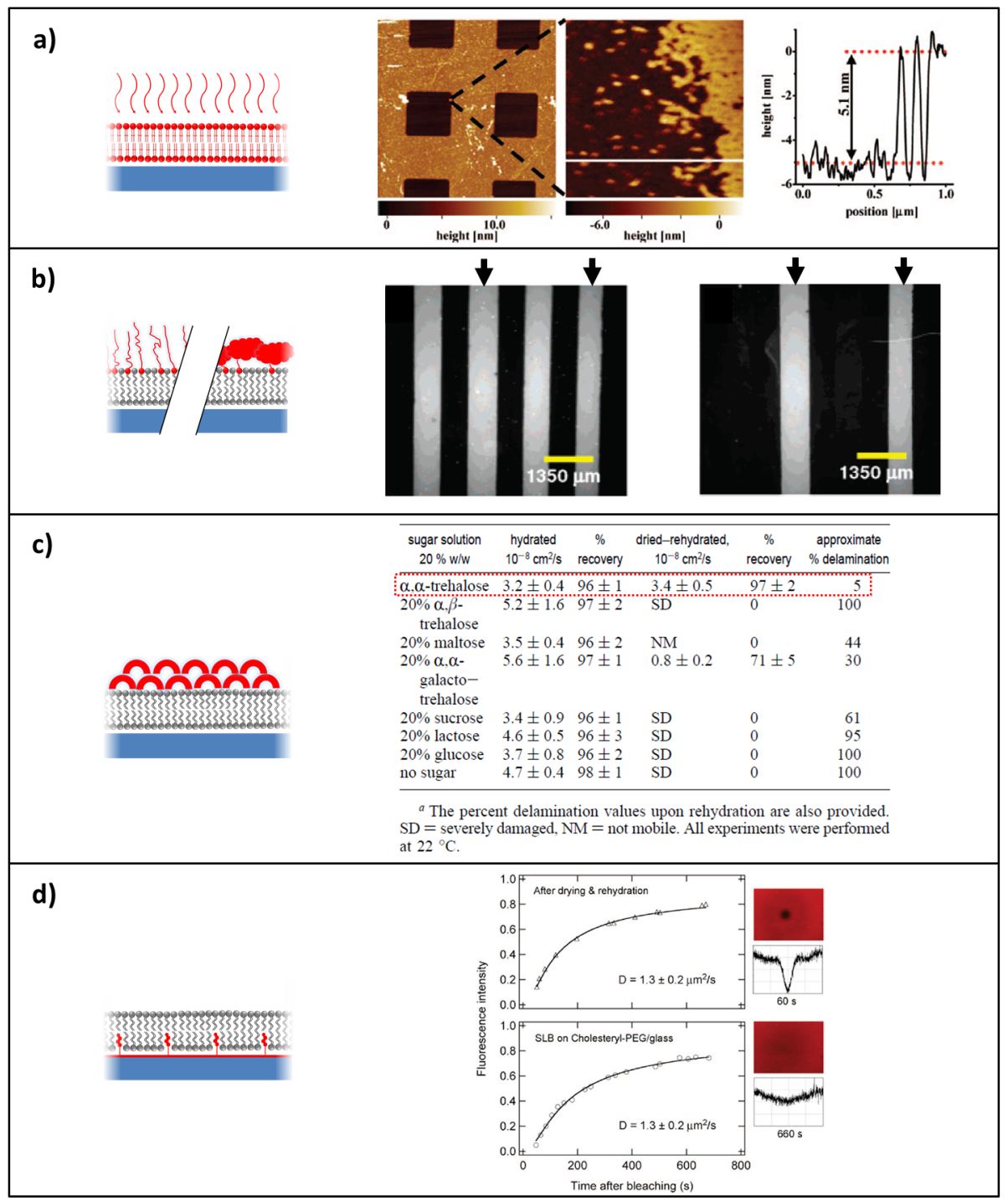

Figure 1.9: Methods to improve air-stability of SLBs; a) use of photocrosslinkable lipids, fabricated patterns remain stable after detergent wash. Reprinted with permission. ${ }^{145}$ Copyright 2009, American Chemical Society. b) Doping the SLB with PEGylated lipids or application of a protein layer was shown to induce air stability after air exposure in a microfluidic device, arrows indicate proteincoated SLBs before and after rehydration. Reprinted with permission. ${ }^{146}$ Copyright 2004, American Chemical Society. c) Trehalose coating was found to induce air stability in SLBs, delamination was estimated to be ca. $5 \%$. Reprinted with permission. ${ }^{150}$ Copyright 2007, American Chemical Society. d) Substrate modification with a homogeneously distributed cholesterol layer was shown to impart air-stability on PEGylated glass slides. Reprinted with permission. ${ }^{155}$ Copyright 2008, American Chemical Society. 


\subsubsection{Leaflet symmetry and composition of SLBs}

Although leaflet composition of SLBs is assumed to be similar to the composition of unilaminar vesicles used to prepare the SLBS, surface-induced re-organization of membrane components has been suggested. When GM1 is included in lipid vesicles and deposited on solid supports, a higher binding of fluorescent cholera toxin was observed in lipid bilayers when compared to lipid monolayers, suggesting enrichment of GM1 in the top leaflet of the lipid bilayer. It was proposed that substrate electrostatically driven rearrangement had occurred. ${ }^{158}$ Additional experiments where Texas Red-labelled lipids were included a larger extent of FRET on GM1-functionalized lipid bilayers was found compared to GM1-functionalized lipid monolayers. ${ }^{158}$ Other studies have suggested substrate-induced leaflet asymmetry in SLBs as well. ${ }^{159}$ Lipid exchange, as observed in vivo, can also be mimicked in SLBs. When positively charged POPC $\%$ POEPC ${ }^{r}$-SLBs were allowed to interact with negatively charged POPC/POPS ${ }^{5}$-vesicles, QCM-D measurements showed an immediate increase in mass and dissipation indicative of absorbed vesicles. Then, the QCM-D response decays back to the baseline indicating that vesicles are detached. During the vesicle residence time on the SLB, lipid exchange is thought to occur until weakening of the electrostatic interaction results in detachment of the vesicle. ${ }^{160}$ Vesicle-membrane interaction will be described in more detail later in section 1.8.2.

\subsubsection{Barrier properties of SLBs}

Active and passive transport of molecules across the SLB can be observed similar to observations made in vivo. Passive transport across the SLB is mostly limited to water molecules diffusing down a concentration gradient. In general, water molecules move from the hypotonic compartment across the SLB towards the hypertonic environment. ${ }^{161}$ The lack of ion transport across an intact SLB is reflected in the high Ohmic resistance of these layers. On the contrary, active transport is facilitated by transport proteins embedded in the cell membrane or reconstituted in SLBs. The action of these proteins requires energy through the consumption of ATP. As a result, ions for example can be transported against the concentration gradient. Recently, both passive and active transport was studied across SLBs that were prepared on a perforated surface with $4 \mu \mathrm{m}$ holes. ${ }^{162}$ These free-standing SLBs were doped with either $\alpha$-hemolysin for passive transport of Alexa-488 dye or proton transporter $F_{0} F_{1}$-ATP synthase for active transport of protons. $^{162}$

In addition, SLBs have been applied in capillary electrophoresis to suppress the occurrence of electro-osmotic flow (EOF). EOF is caused by the negatively charged silanol-groups on

\footnotetext{
${ }^{9}$ POPC: 1-palmitoyl-2-oleoyl-sn-glycero-3-phosphocholine

'POEPC: 1-palmitoyl-2-oleoyl-sn-glycero-3-ethylphosphocholine

${ }^{s}$ POPS: 1-palmitoyl-2-oleoyl-sn-glycero-3-phospho-L-serine 
the glass surface of the capillaries and can result in a flow of ions when an electric field is applied. The neutral SLB overcomes the Debye screening length inside the capillary and provides a net neutral surface charge avoiding EOF. In one such study, the EOF suppression of gel and liquid-state SLBs was evaluated. It was found that gel-state SLBs are more stable resulting in higher EOF suppression. ${ }^{163}$

\subsection{Introducing bio-functionality in SLBs}

Introducing functionality to the surface of SLBs can be achieved following a variety of modification strategies summarized in Figure 1.10. Reversible and irreversible membrane association can be distinguished and means to control the orientation of the functional ligand on SLBs can be important. In this section selected strategies to introduce biofunctionality to SLBs are presented, while complementary information can be found in an earlier review of Groves and co-workers. ${ }^{35}$

His-tagged ligands can be bound in a supramolecular fashion to an NTA head-group of lipids that are present in the SLB. Coordinated $\mathrm{Ni}^{2+}$ metal ions by NTA displays high selectivity and moderate affinity towards His-tags. The association constants of His-tagged proteins to NTA-functionalized SLBs was found to be $10^{4} \mathrm{M}^{-1} .{ }^{164}$ To preserve a proper orientation and stable attachment multiple histidine residues can be introduced in the ligand of interest. For example, $\mathrm{His}_{6}$-tagged major histocompatibility complex (MHC) and intercellular adhesion molecule 1 (ICAM-1) were presented on mobile SLBs for the formation of a hybrid immunological synapse (IS). These synapses were biologically active and able to induce the characteristic T-cell synapse bull's eye formation. ${ }^{165}$ Removal of the bound ligands can be achieved by either addition of a competitor, like imidazole, or depletion of $\mathrm{Ni}^{2+}$ by ethylenediaminetetra-acetic acid (EDTA) that is able to sequester bivalent metal ions.

High affinity association constants of up to $10^{15} \mathrm{M}$ can be reached between biotinylated lipids in the SLB and streptavidin, neutravidin and avidin. ${ }^{166}$ The use of fluorescently labelled neutravidin allows for direct monitoring of lateral mobility of ligands and quantification of the amount of molecules per surface area. ${ }^{167}$ Antibodies associated to the SLB in this manner have been used to immobilize ligands to SLBs for cancer cell purification. ${ }^{129}$ In this case, biotinylated antibodies directed against cancer cells were assembled on a neutravidin-modified SLB. These surface were used to collect cancer cells from blood samples. 


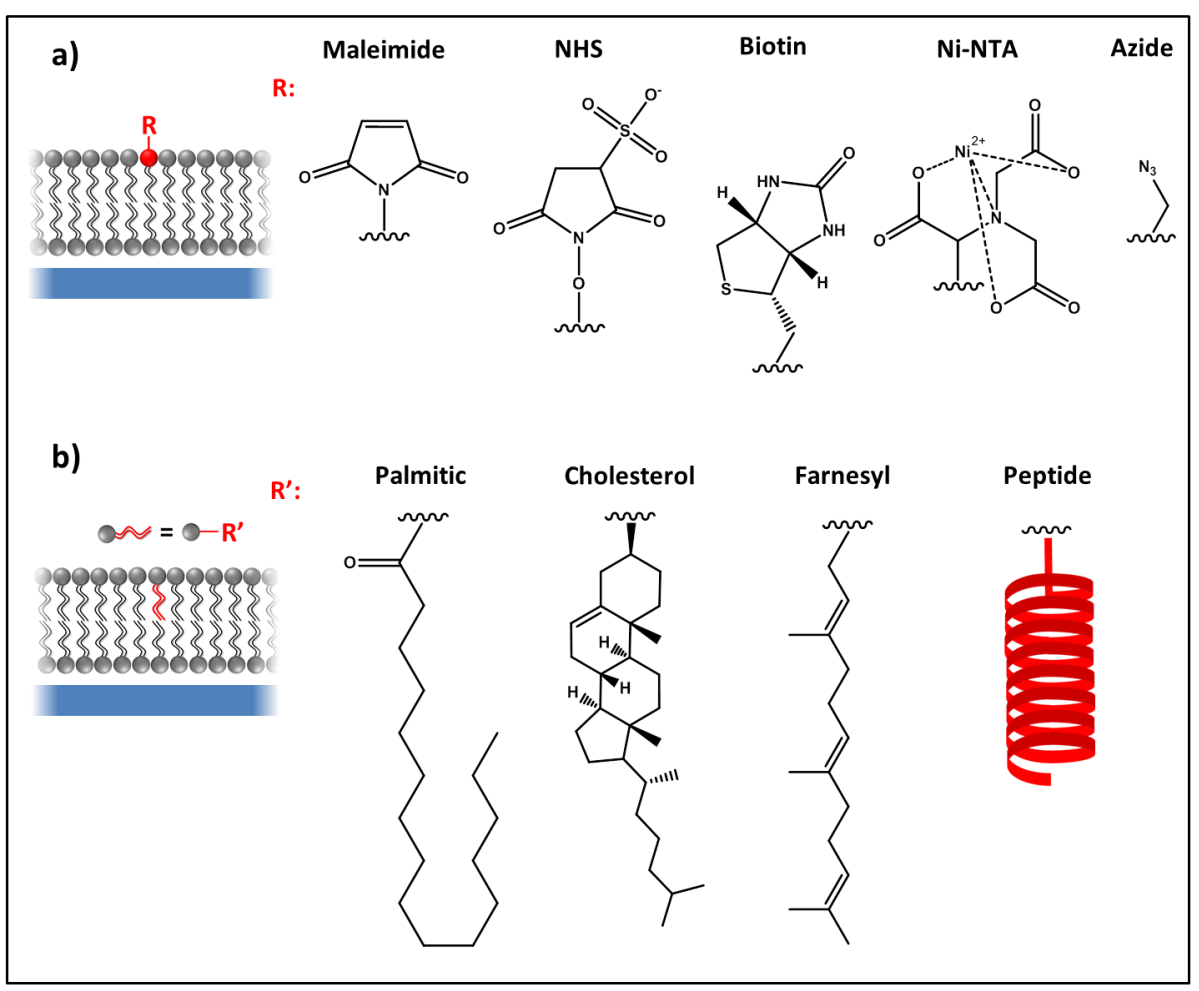

Figure 1.10: Selected modification strategies of SLBs; a) using lipid head-groups bearing maleimide, NHS, biotin, Ni-NTA or azide functionalities. b) using amphiphiles using various lipid anchors, for example palmitic, cholesterol, farnesyl or $\alpha$-helical peptides.

Apart from the non-covalent modification of SLBs, covalent coupling of ligands to SLBs can be achieved using e.g. maleimido-functionalized lipids, which can react under mild conditions with e.g. cysteine residues. ${ }^{168}$ For example, $\mathrm{CGGH}_{6}$ peptides were conjugated to maleimide-bearing SLBs and used for the association with Ni-NTA containing vesicles. ${ }^{169}$ Other chemical functional groups such as carboxylic acids in DP-NGPE ${ }^{t}$ lipids embedded within an inert SLB were activated with $\mathrm{N}$-hydroxysuccinimide (NHS). The resulting NHSactivated lipids was used for further reaction with amine-functionalized ligands such as lysine residues of collagen type I. ${ }^{170}$

\footnotetext{
${ }^{t}$ DP-NGPE: 1,2-dipalmitoyl-2-oleoyl-sn-glycero-3-phosphoethanolamine-N-(glytaryl) 
Azido-functionalized lipids can be used to 'click' alkyne-functionalized ligands to SLBs. For example, Boxer and co-workers attached alkyne-DNA to POPE- $\mathrm{N}_{3}{ }^{u}$. The DNA presenting SLB was used for the study of vesicle binding to SLBs by means of DNA hybridization. ${ }^{171}$

Instead of chemical modification of SLBs, ligands can be modified with anchoring groups that allow for insertion into the SLB. This insertion resembles the in vivo insertion of lipidated proteins into the cellular membrane. A common in vivo lipid modification of proteins is prenylation, i.e. the addition of an isoprenoid, either a farnesyl or a geranylgeranyl group, to a C-terminal cysteine. ${ }^{172}$ Three enzymes are known to catalyze prenylation of proteins; farnesyltransferase (FTase), geranylgeranyltransferase type I (GGTase-I) and geranylgeranyltransferase type II (GGTase-II), also known as Rab GGTase. FTase uses farnesyl pyrophosphate (Fpp) as the prenyl donor, and both GGTase-I and Rab GGTase use geranylgeranyl pyrophosphate (Gpp). ${ }^{173}$ In vivo prenylation occurs at the socalled tetrapeptide sequence $\mathrm{CaaX}$ ( $C$ is cysteine, $A$ is an aliphatic amino-acid and $X$ is any amino-acid) where $X$ can be modified with farnesyl or geranylgeranyl group. ${ }^{174,175}$ It is shown that proteins could be farnesylated in vitro as well as in vivo. In vitro farnesylation can be carried out by addition of FTase enzyme and substrate. Much more elegant is the single step in vivo farnesylation of the CaaX box directly in $E$. coli cell lysate. Since bacteria lack the capacity for protein prenylation, the bacteria were transformed with a vector inducing the expression of both the target protein and the FTase enzyme subunits. This resulted in the production of recombinant farnesylated proteins using $E$. coli's natural Fpp pool. ${ }^{175}$ Another lipidation reaction is myristoylation, which introduces the fatty acid myristate to the $\mathrm{N}$-terminal amino acid glycine in susceptible proteins. The reaction is mediated by $\mathrm{N}$-myristoyltransferase (NMT) that recognizes the $\mathrm{N}$-terminal MGxxS/Txx motif, where $x$ indicates any amino acid that couples a myristoyl group to the $\mathrm{N}$ terminus. ${ }^{176,177}$ Palmitoylation, catalysed by protein acyltransferases (PATs), results in the transfer of a palmitate to a protein. ${ }^{174}$ In vitro palmitoylation has been used for the preparation of peptide amphiphiles assembled into monolayer for use in cell culture. ${ }^{178}$

Glycosylphosphatidyinositol (GPI) anchors membrane proteins to the extracellular face of the plasma membrane. ${ }^{179}$ Ligands with exposed GPI anchors can be incorporated in SLBs via insertion of the GPI tail into the lipid bilayer. Although widely used in the past, problems related to unnatural aggregate formation and GPI-protein purification, makes this technique much more cumbersome than other available methods. ${ }^{35,180}$

Another possibility is using cholesterol derivatives as lipid anchors. As was explained previously, cholesterol has a stabilizing effect on lipid bilayers and it can readily associate

\footnotetext{
${ }^{u}$ POPE-N $\mathrm{N}_{3}$ : Azido 1-palmitoyl-2-oleoyl-sn-glycero-3-phosphoethanolamine
} 
with the hydrophobic core of the lipid bilayer. A more comprehensive overview on cholesterol anchors and tethers can be found elsewhere. ${ }^{181}$

Depending on its primary amino-acid sequence, a number of peptides have the natural propensity to penetrate into a lipid bilayer usually in an $\alpha$-helix conformation. Insertion of WALP containing peptides into the bilayer has been elucidated in silico where it can be described as a three step process, i.e. (1) insertion of one or more tryptophan residues within the lipid bilayer below the phosphate groups, (2) further insertion into the bilayer in an unstructured state and (3) assembly into an $\alpha$-helix nearly perpendicular to the plane of the membrane to provide a stable anchoring point facing away from the SLB into the solution. ${ }^{182}$ The membrane association potential of $\alpha$-helix peptides was studied that pass the bilayer only once. ${ }^{183}$ However, utilization of WALP peptides to anchor ligands to SLB, to our knowledge, remains unexplored.

Reconstitution and incorporation of complete membrane proteins in the SLB can be another way to prepare bio-functionalized SLBs. ${ }^{184}$ As stated previously, substrate-protein interaction can however occur once proteins embedded within an SLB and result in a loss of protein function. As a consequence, studies on transmembrane proteins in SLBs are often mostly performed on tethered SLB systems. ${ }^{185}$ When the membrane protein is solubilized with a detergent and mixed with detergent-destabilized liposomes, after removal of the detergent, the protein will be inserted into lipid bilayer of the proteoliposomes that can be used for SLB formation. For more information recent reviews by Martin and Williams and co-workers can be consulted. ${ }^{36,186}$

Although a variety of lipid anchors have been described, the stability of incorporation in the SLB does vary. In a recent study, stability characteristics of oligonucleotides to DOPCbased SLBs was studied using cholesterol, stearyl and di-stearyl lipid anchors. ${ }^{187}$ QCM-D experiments revealed that single stranded (ss)DNA anchored via cholesterol or stearyl results in unstable anchoring. Hybridization of complementary ssDNA conjugated to cholesterol or stearyl provides stable insertion into the bilayer. The di-stearyl anchored ssDNA provided resistance to rinsing the bilayer without the need for hybridization to a complementary anchor. ${ }^{187}$ As a result, the stability of inserted lipids must be considered when deciding upon a lipid anchor. The choice of tether that bridges the ligand from the substrate, in this case the SLB, to the target can play a key role. This is particularly important when considering live cell experiments. A recent paper describes the effect of the linker on the $I C_{50}$ value $^{v}$ and the biological response of cells. In short, the proposed polyproline linker outperformed the 'industry standard' PEG linker. Improved cell

\footnotetext{
${ }^{\vee} \mathrm{IC}_{50}$ : Value of half maximal inhibitory concentration for a specific biological or biochemical function 30
} 
adhesion kinetics were observed when the linker was modified with a cell adhesive peptide. $^{188}$

\subsection{Bio-analytical applications of SLBs}

\subsubsection{Membrane electrophoresis}

The fluidic nature of lipids in membranes is an interesting property to exploit for conducting membrane electrophoresis experiments either on the cell membrane itself or, more recently, on SLBS (Figure 1.11). ${ }^{189,190}$ For example, it has been shown that zwitterionic glass-supported SLBs doped with negatively charged and fluorescent lipid-dye conjugates can be separated under an electric field. ${ }^{190,191}$

To achieve SLB electrophoresis several aspects need to be taken into consideration such as the height of the aqueous compartment above the SLB, the distance between the electrodes and the solution conductivity in order to achieve a sufficiently large electric field (E-field). Since traditional electrophoresis set-ups rely on bulky electrodes and high applied DC potentials (10-100 Vs), bubble formation, pH changes and even Joule heating are known to occur. Cremer and co-workers have described an elegant way of minimizing these effect by the fabrication of a fluidic device that provides a continuous flow over the electrodes to wash away unwanted by-products (Figure 1.11a). Using this electrophoresis platform, the electro-osmotic motion of streptavidin bound to the SLB could be tuned with $\mathrm{pH}$ and ionic strength. In their case the extent and direction of the mobility of streptavidin could be altered, e.g. a pH closer to the $\mathrm{pl}$ resulted in limited electro-osmotic mobility. ${ }^{192}$ This work was expanded to achieve electrophoretic-electro-osmotic focusing (EEF) of streptavidin with different charges bound to the SLB. ${ }^{193}$

Although these bulk electrophoresis systems have been used with great success, they still inherently suffer from the high applied potentials required to drive the mobility. In our group we proposed an $\mu S L B$ electrophoresis system that utilizes potentials below the electrolysis potential $(1.23 \mathrm{~V})$ of water (Figure 1.11b). To this end, we designed a microfluidic chip with three electrode junctions with an inter-electrode spacing of $500 \mu \mathrm{m}$ (Chapter 2). The fluidic channels were $500 \mu \mathrm{m}$ in width and $50 \mu \mathrm{m}$ in height. Moreover, the junctions were patterned with $\mathrm{Cr}$-barriers enabling multiplexing. To overcome the lack of Faradaic currents, we included a sacrificial electro-active compound to enable the generation of an electric field. (hydroxymethyl)-Ferrocene $\left(\mathrm{F}_{\mathrm{c}} \mathrm{CH}_{2} \mathrm{OH}\right)$ was chosen based on its electro-neutrality and inertness to the SLB and proteins. Upon addition of $0.5 \mathrm{mM}$ $\mathrm{F}_{\mathrm{c}} \mathrm{CH}_{2} \mathrm{OH}$ electrophoretic migration of TR-DHPE was noted at potentials as low as 250 $\mathrm{mV}^{194}$ 


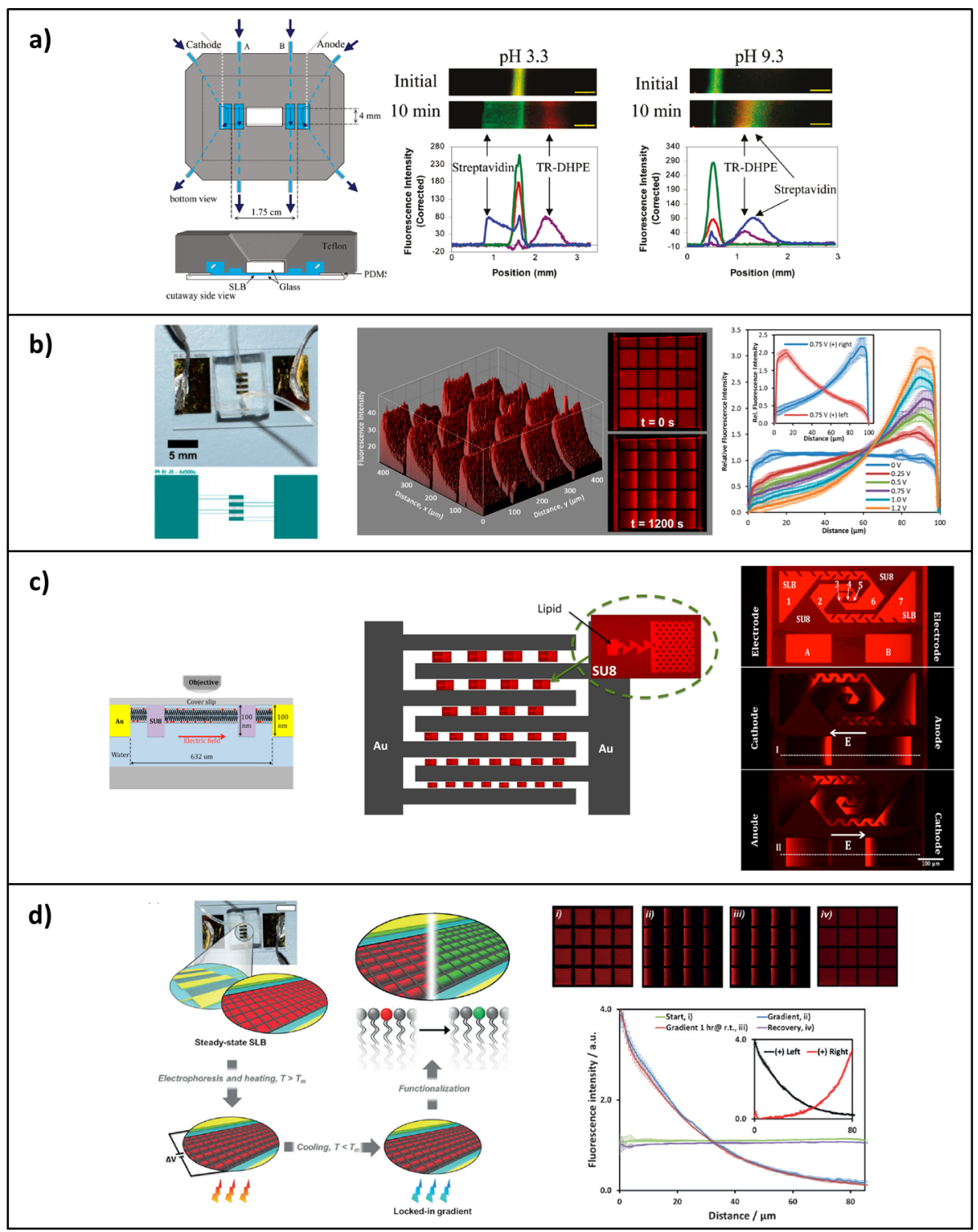

Figure 1.11: Overview of several SLB electrophoresis approaches; a) fluidic device developed by the Cremer and co-workers to mitigate electrolysis by-products and enable the use buffered conditions in DC electrophoresis. Reprinted with permission. ${ }^{192}$ Copyright 2011, American Chemical Society. b) On-chip DC and AC $\mu S L B$ electrophoresis device using low potentials and a sacrificial reaction enabling the use several 100s of millivolts. Reprinted with permission. ${ }^{194}$ Copyright 2014, American Chemical Society. c) On-chip ratchet SLB electrophoresis device for high concentration build-up of charged analytes. Reprinted with permission. ${ }^{195}$ Copyright 2012, American Chemical Society. d) The use of SLB electrophoresis to prepare stable locked-in surface gradients in gel-state SLBs. Reprinted with permission. ${ }^{196}$ Copyright 2014 , John Wiley \& Sons. 
In all the aforementioned cases, removal of the electric field results in the recovery of the initial steady-state due to the lipid diffusivity. The use of AC electric fields in combination with surface ratchets proved effective in retarding the lateral mobility of lipids in the SLB as shown for micro-contact printed ratchets and a chip-based device (Figure 1.11c). ${ }^{195,197,198}$ On the other hand, Cremer and co-workers demonstrated lipid fixation by freezing the surface after a complex SLB was used for membrane electrophoresis. Freezedried SLBs can be used in set-ups such as MALDI mass spectroscopy for further chemical analyses. ${ }^{118}$ Temporal stability can also be achieved by the use of gel-state lipids (Figure 1.11d). ${ }^{196}$ We recently demonstrated that conducting electrophoresis above the $T_{m}$ and after rapidly cooling down till below the $T_{m}$ locked-in SLB gradients can be fabricated that are tuneable in surface chemistry (Chapter 3 ).

Movement of membrane bound components can be achieved solely by shear stress induced by bulk fluid flow. In a recent example, photo-crosslinkable DiynePC lipid were used to prepare 2D porous lipid network. This was made possible by varying the UVillumination used to crosslink the DiynePC SLB. After crosslinking, monomers were washed away and the exposed regions were backfilled with a DOPC-based SLB functionalized with biotin. Upon binding of dye-labelled streptavidin to these biotin-SLBs, front velocities of streptavidin and a labelled lipid were compared after applying bulk flow. In both cases, a linear dependency was found between the increase in applied UV-dose and the decrease in front velocity. ${ }^{199}$ The use of hydrodynamic forces to separate membrane bound species has also been investigated in pure liquid-state SLB, as demonstrated by Höök and coworkers (Figure 1.12a). ${ }^{200,201}$

\subsubsection{Studying membranes processes using SLBs}

Inspired to mimic natural SNARE-mediated fusion (Figure 1.12b). ${ }^{202}$ Boxer and co-workers studied the hybridization of ssDNA-functionalized SLBs to complementary DNA on lipid vesicles (30-200 $\mathrm{nm}$ in diameter). ${ }^{203}$ However, instability of the tethers in the underlying SLB was found. This led to the investigation of the influence of SLB composition on the immobilization of lipid vesicles via DNA hybridization. It was found that the use of gelstate SLBs with cholesterol, more stable SLBs were made resulting in a more stable tethering of the vesicle to the SLB, suggesting a role of membrane stiffness on this process. ${ }^{204}$ Stable tethering of lipid vesicles using DNA hybridization can furthermore be achieved by using self-assembled-monolayers (SAMs) as a support for SLB formation. In this case, the SAM is modified with SSDNA that can hybridize with SSDNA-functionalized GUVs. As a result, the GUVs will rupture to yield an SLB patch comprising of liquid-state lipids presenting ssDNA on the distal leaflet. ${ }^{205}$ Fusion of tethered vesicles could be observed on these layers. In this case, DNA-tethered vesicles loaded with $100 \mathrm{mM}$ of selfquenched calcein were used. Upon hybridization with the planar SLB, fusion can be 
monitored by calcein dequenching induced by dilution and diffusion of the dye into the aqueous compartment below the planar SLB (Figure 1.12b). ${ }^{206}$ Vesicle fusion with native cell membranes can be used to insert functional groups into the cell membrane for bioorthogonal modification of the cell surface. ${ }^{207,208}$

In addition, membrane fusion has been induced by the edge of a hydrodynamically driven SLB within a microfluidic device to facilitate rupture of adsorbed lipid vesicles of nonfusogenic composition as was studied by Höök co-workers. Recently, this process was shown for non-fusogenic vesicles directly derived from NH3T3 cells. ${ }^{43}$ Content transfer was validated by cholera toxin binding to GM1.

\subsubsection{Biosensing and analysis of membrane proteins}

Over $60 \%$ of known disease markers are membrane proteins. Moreover, $20-30 \%$ of the genes that encode for proteins are found in lipid membranes. ${ }^{209}$ This has motivated research into studying membrane proteins in their native membranous environment by using model membranes. For example, heavily doped-silicon nanowires coated with an SLB doped with $\alpha$-hemolysin were fabricated to study the transport of $\mathrm{Fe}(\mathrm{CN})_{6}{ }^{4-210,211}$ Array-based SLB systems have been proposed and reviewed by Vörös and co-workers and others. $^{52,53,59-61,212}$ For instance, SLBs formed on a porous nanoarray doped with the gramicidin-D ion channel could be measured using impedance spectroscopy. ${ }^{213}$ More recently, arrays of free standing SLBs have been used to monitor active and passive transport across $\alpha$-hemolysin (Figure 1.12c) and $F_{0} F_{1}$-ATP synthase, respectively. 


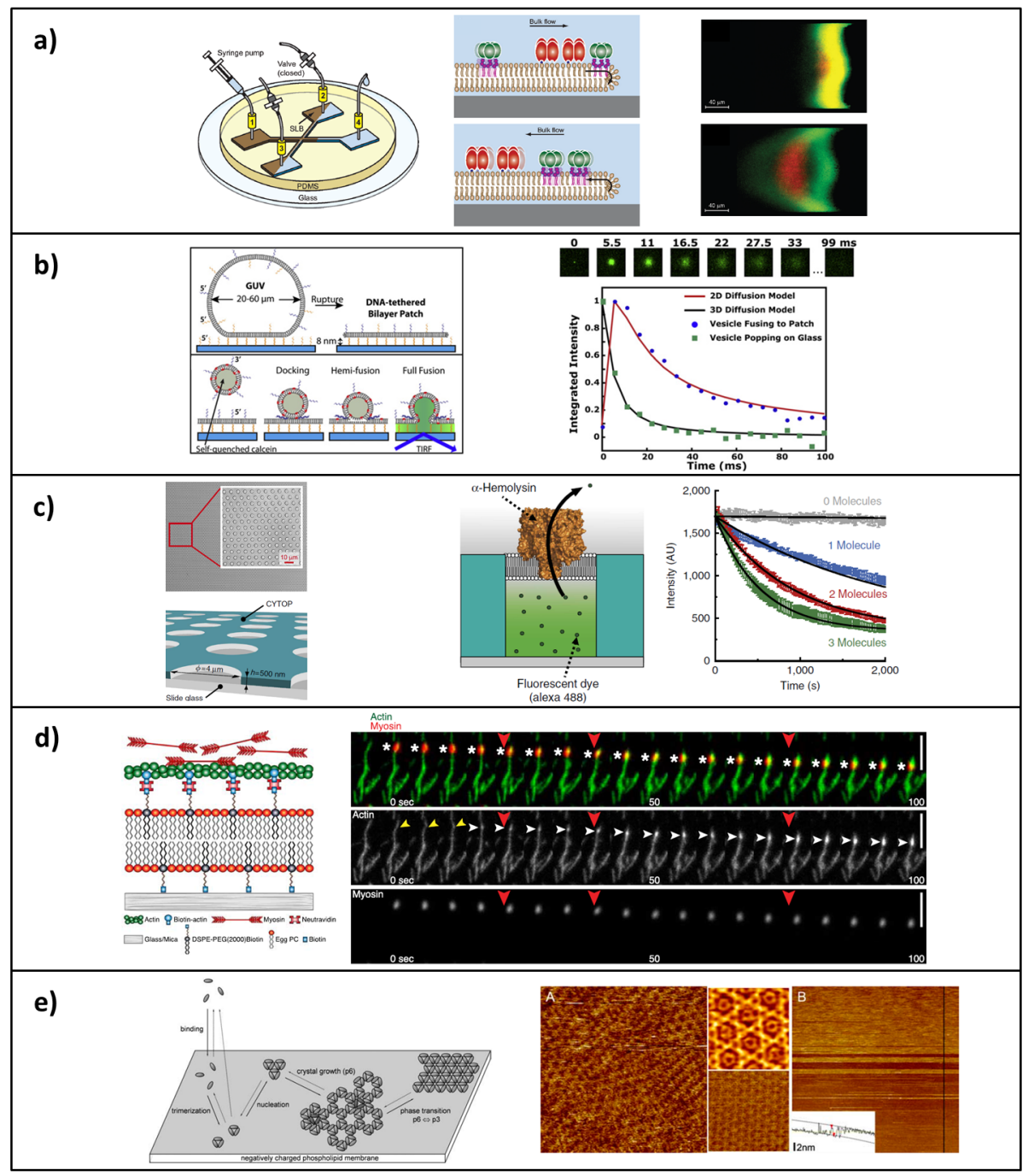

Figure 1.12: Studying membrane composition and membrane processes; a) fluidic device developed by Höök and co-workers for the separation of membrane bound components by hydrodynamic force. Reprinted with permission. ${ }^{200}$ Copyright 2011, American Chemical Society. b) The use of planar SLBs to tether lipid vesicles by means of DNA hybridization, showing content transfer across the SLB by calcein dequenching. Reprinted with permission. ${ }^{206}$ Copyright 2011, Cell Press. c) Preparation of an array of free-standing SLBs doped with $\alpha$-hemolysin to study the passive transport of Alexa 488 dye. Reprinted with permission. ${ }^{162}$ Copyright 2014, Nature Publishing Group. d) Fabrication of a minimal actin cortex on SLBs and the study of myosin-induced contractility and tension build-up. Reprinted with permission. ${ }^{214}$ Copyright 2013, elife Sciences Publications. e) The self-assembly of Anx5, involved in membrane repair, on negatively charged SLBs in the presence of $\mathrm{Ca}^{2+}$ and the formation of 2D protein crystals, observed on the lipid bilayer with AFM. Reprinted with permission. ${ }^{215}$ Copyright 2005, Cell Press. 


\subsubsection{Cytoskeletal organization}

SLBs have been used as a minimal system for studying cell membrane-cytoskeleton interactions as has been recently reviewed. ${ }^{216-218}$ For example, actin filaments meshwork's have been prepared on SLBs. Upon introduction of ponticulinw, isolated from Dictyostelium discoideum in the SLB, addition of both monomeric G-actin and filamentous F-actin resulted in a network of actin fibres on the SLB surface. ${ }^{219} \mathrm{~F}$-actin association to the membrane can be mediated by the enzyme ezrin. This enzyme can be changed from a dormant into an active state by either phosphorylation or by binding to $\mathrm{L}-\alpha$ phosphatidylinositol-4,5-biphosphate $\left(\mathrm{PiP}_{2}\right)$. When $\mathrm{SLBs}$ were doped with $\mathrm{PiP}_{2}$, filopodialike structures were formed in the presence of a cocktail of proteins to allow for the formation of dendritic actin-like assemblies. Subsequent addition of a frog extract resulted in the formation of these filopodia-like architectures. ${ }^{220}$ Self-assembly of actin filaments has also been shown on lipid vesicles ${ }^{221}$ and anchoring of actin filaments inside GUVs. ${ }^{222}$ Recently, Schwille and co-workers were able to use an SLB-actin network to track contractility induced by myosin along the actin fibres (Figure 1.12d). ${ }^{214}$ Here a minimal actin cortex was reconstituted on the SLB and fluorescent myosin was tracked in the presence of ATP. Based on their findings, they postulate that buckling and breakage of filaments is involved in compaction and in the build-up of tension, turnover of actin and reorganization of the cortex. ${ }^{214}$

\subsubsection{Membrane repair}

Brisson and co-workers made use of SLBs to help elucidate the process of cell membrane repair. Annexin A5 (Anx5) is a protein known to bind to DOPS ${ }^{x}$ lipids in the presence of $\mathrm{Ca}^{2+}$. Early on, it was found that Anx5 forms 2D crystals on DOPS-doped SLBs in the presence of $\mathrm{Ca}^{2+}$, as observed with AFM (Figure 1.12e). ${ }^{223}$ Formation of these crystals was suggested to occur in a stepwise manner. First binding to a negatively charged SLB followed by formation of a trimer, nucleation and crystal growth. Transition from a low to a high density could be quantified with a difference of $36 \mathrm{ng} / \mathrm{cm}^{2}{ }^{215}$ Based on these findings, it was postulated that Anx5 forms a 2D crystal in vivo upon membrane damage. The resulting influx of $\mathrm{Ca}^{2+}$ ion outside the cell would allow intracellular Anx5 to interact with PS lipids present in the cell's proximal leaflet to locally 'heal' the cell membrane. After closure, membrane resealing by cytoplasmic vesicles would occur. This was proven correct with in vitro analysis of cell membrane repair in mouse perivascular cells. ${ }^{224}$ Comparison between wild-type and Anx5 knock-out cells confirmed the role of Anx5.

\footnotetext{
${ }^{\mathrm{w}}$ Ponticulin: transmembrane protein, anchor for actin filaments and nucleation site for actin assembly in vivo.

${ }^{x}$ DOPS: 1,2-dioleoyl-sn-glycero-3-phospho-L-serine 


\subsection{SLBs as an interface to cells}

\subsubsection{Mimicking cell-cell contacts}

Cadherins play important roles in cell adhesion forming adherens junctions to bind cells together. This is a highly dynamic process and the actin cytoskeleton is directly modulated by the adhesive strength and clustering of cadherins. In depth investigation into the mobility of proteins associated with model membranes was performed with human Ecadherin. The extracellular domain of human E-cadherin, appended with Fc domain of human IgG, was fused to a GPI lipid bilayer anchor (hEFG) and evaluated on mobile PC SLBs. The hEFG fusion protein displayed a lateral mobility of $0.6 \mu \mathrm{m}^{2} / \mathrm{s}$ with a substantial immobile fraction (30-60\%). Since SLBs allow for ligand presentation that display lateral mobility, similar to natural systems, the presence of large immobile fractions is undesired. An elegant way to separate mobile from immobile hEFG was accomplished by membrane electrophoresis. Using this approach, the two fractions could be identified and collected. Even though more than half of the hEFG complex remained immobile, $30 \%$ of MCF- $7^{y}$ cells were able to cluster the proteins embedded in the SLB. However, lack of ECM immobile anchoring points caused the cells to be loosely adherent and display a spheroid morphology. This resulted in a large population of non-viable cells, as a result only $30 \%$ hEFG clustering could be observed. ${ }^{225}$

The importance of retaining ligand mobility in mimicking cell-cell contacts was more convincingly shown for the epidermal growth factor receptor tyrosine kinase (EGFR). The EGFR is a member of the type I, ErbB, receptor kinase family that is known to be deregulated in human cancers. ${ }^{226}$ Using mobile SLBs, decorated with epidermal growth factor (EGF) the effect of mobility on immortal, nontransformed MCF-10a ${ }^{2}$ cells was studied. ${ }^{227}$ Mobile DMPOC ${ }^{\text {aa }}$ SLBs were doped with 3 mol\% biotinylated lipids that were used to associate EGF via biotin-streptavidin interactions to the SLB. Upon analysis of the lateral mobility, a higher mobility was observed for fluorescently labelled lipids in comparison with the EGF-Lipid complex. After 20 hours of MCF-10a cell culture, specific attachment of cells was noted only on EGF-modified SLBs and not on the control surfaces. The specificity of the interaction was demonstrated with a competition assay using mAb225, which is a competing antibody for EGFR ligand binding. A reduction in cell count of $94 \%$ was observed in the presence of $3 \mathrm{ng} / \mathrm{mL}$ mAb225. Involvement of EFGR kinase activity in cell EGF-EGFR mediated cell adhesion was proven by addition of a specific kinase inhibitor, Tarceva. During cell culture distinct EGF clusters were formed during the 20 hour culture period, reminiscent of integrin focal adhesions. The clusters were found to

\footnotetext{
${ }^{y}$ MCF-7: Michigan Cancer Foundation-7, breast cancer cell line

${ }^{2}$ MCF-10a: human breast epithelial cells

${ }^{\text {aa }}$ DMOPC: 1,2-dimyristoleoyl-sn-glycero-3-phosphocholine
} 
be out-of-plane compared to the SLB, suggesting that the natural endocytotic functioning of EGF is retained on the SLBs. To study whether clustering of EGF was the direct result of membrane fluidity, a comparison was made with DPPC-based SLBs ( $T_{m} 41^{\circ} \mathrm{C}$ ), which display a significantly lower lateral mobility of at least one order of magnitude. In this case, less cell spreading was observed indicative of a less motile phenotype and less EGF clustering. Taken together, the results illustrate that dynamic, local enrichment of EGF and its receptor is dependent on the lateral mobility of the ligand. ${ }^{227}$

\subsubsection{Supporting cell differentiation on SLBs}

Another study investigated the immobilization of 'pluripotent tissue sheets' on SLBs to regulate cell differentiation. $N C C^{\text {bb }}$ cell differentiation is important in vertebrate development. Instead of single cells, explanted cohesive cell sheets obtained from Xenopus laevis were used. The isolated animal cap, from Xenopus blastula stage embryos, consists of a cohesive cell sheet of pluripotent cells, similar to mammalian embryonic stem cells. Cultivation of these pluripotent tissue sheets on unmodified zwitterionic SOPC ${ }^{c c}$ SLB resulted in tissue dissociation and cell death due to poor cell-substrate interaction. The importance of presenting proper cues to maintain tissue structure was illustrated when RGD and Xenopus cadherin 11 (Xcad-11) were compared. The RGD amino-acid motif, found in the tenth fibronectin type III domain has been used extensively to engage on $\alpha_{v} \beta_{3}$ integrin receptors. ${ }^{228-230}$ The RGD motif has been explored in cancer medicine to block the often overexpressed $\alpha_{v} \beta_{3}$ integrin, in particular cyclic RGD peptides show improved interaction with the receptor. ${ }^{231}$ On the other hand, immobilization of these peptides has enabled cell growth on otherwise cell unfriendly materials. ${ }^{232}$ Although strong integrinmediated adhesion of NCCs to the SLB surface was observed in the case of RGD, the cells lost their connective structural integrity resulting in cell death. Tissue cohesiveness was maintained when Xcad-11 was presented to the animal caps that were induced for neural crests. This observation was attributed to a switch in cadherin profile. Wild-type animal caps could only be maintained on a E-cadherin modified SLB. Moreover, this behaviour was not only strongly dependent on the type of ligand, but also on the interligand spacing with an observed optimum of ca. $5.7 \mathrm{~nm} .^{233}$

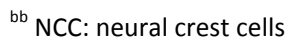

${ }^{c c}$ SOPC: 1-stearoyl-2-oleoyl-sn-glycero-3-phosphocholine 


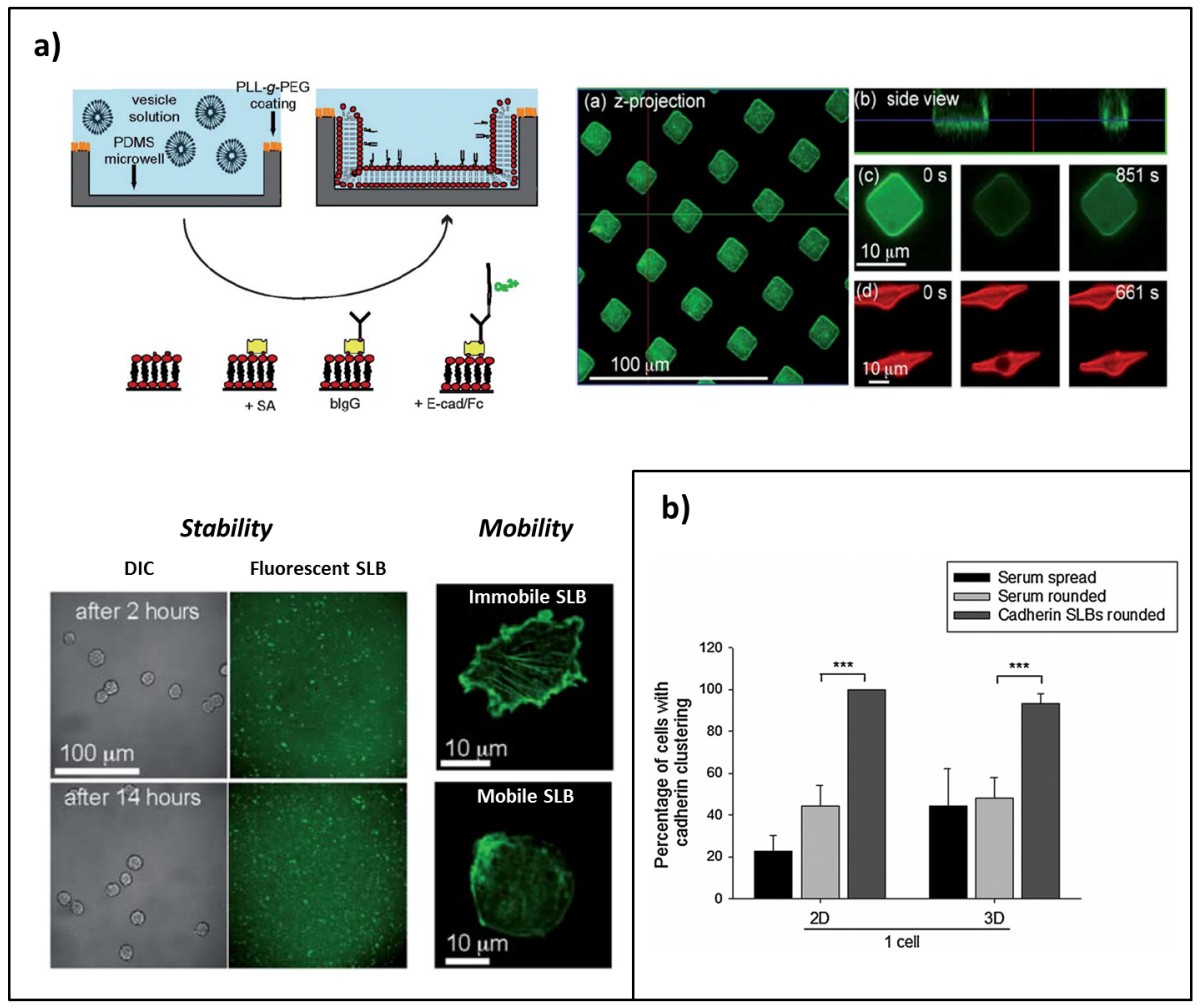

Figure 1.13: Mimicking cell-cell contacts on SLBs; a) SLBs were modified with E-cadherin and deposited on PDMS microwells for 3D presentation of mobile ligands to cells. Reprinted with permission. ${ }^{131}$ Copyright 2011, Royal Society of Chemistry. b) Effect of 2D vs. 3D on E-cadherin clustering in MCDK-cells. Reprinted with permission. ${ }^{234}$ Copyright 2012, Royal Society of Chemistry.

Tissue formation during morphogenesis occurs via self-assembly of pluripotent cells. The initial step in this process is the generation of a tissue template determined by the spatiotemporal patterning of cells. This step relies heavily on the expression of cell adhesion proteins, like the ones belonging to the cadherin family. In particular $\mathrm{N}$-cadherin is known to be involved in the formation of junctional cell-cell contacts. Moreover, it has been implicated in mesenchymal condensation during limb bud, cartilage and skeleton development. $\mathrm{N}$-cadherin modified SLBs were used to elucidate and guide emergent tissue architectures by PDC ${ }^{\text {dd }}$ cells. Here, NTA-modified SLBs were chelated with $\mathrm{His}_{6}$-tagged NCadherin. ${ }^{235}$ Successful modification of the SLB was confirmed using QCM-D. In general, less cell spreading was noted for PDCs seeded on SLB, with or without N-cadherin, compared to glass controls. At low seeding densities PDC acquired a spheroid morphology

\footnotetext{
${ }^{\text {dd }}$ PDC: primary adult periosteum derived multipotent cells
} 
on $\mathrm{N}$-cadherin modified SLBs while at high seeding densities cells tended to form aggregates. In the latter case, down regulation of junctional proteins $\mathrm{N}$-cadherin $(\mathrm{CDH} 2$ gene) and ZO-1 (TJP1 gene), matricellular protein Periostin (Postn gene) and markers for mesenchymal condensation and early lineage commitment to osteochondral and adipogenic fates was found. This, in combination with up-regulation of the gene for hyaluronic acid (HA3 gene) concomitant with down-regulation of CD44, (hyaluronic acid receptor) suggest a pre-condensation state of PDCs grown at high density on N-cadherinmodified SLBs. In contrast, unmodified SLBs drive PDCs towards the osteochondral lineage with associated up-regulation in markers genes. ${ }^{235}$ This study highlights the potential of SLBs to investigate the mechanisms involved in tissue development and homeostasis.

\subsubsection{D presentation of ligands using SLBs}

Studying cellular behaviour on a single in vitro platform thereby mimicking the threedimensional environment of neighbouring cells has been shown by Reimhult and coworkers (Figure 1.13). $\mathrm{CHO}^{\text {ee }}$ cells were cultured in SLB-coated PDMS microwells to enable a $3 \mathrm{D}$ presentation of the extracellular domain of E-cadherin. MPPC ${ }^{\mathrm{ff}}$ lipids with a $T_{m}$ of $35^{\circ} \mathrm{C}$ were used to switch between a mobile and immobile presentation of E-cadherin by changing the cell culture temperature. The SLB was modified with streptavidin and biotinylated-antibodies to capture the extracellular domain of E-cadherin. Under normal cell culture conditions $\left(37^{\circ} \mathrm{C}\right)$ the $\mathrm{CHO}$ cells experienced mobile ligands while cells cultured at lower temperatures $\left(30^{\circ} \mathrm{C}\right)$ interacted with immobile E-cadherin. Analysis of the actin cytoskeleton showed that stress fibre formation was impaired on the mobile SLB, while immobile SLBs showed the presence of distinct actin bundles after 14 hours of cell culture (Figure 1.13a). ${ }^{131}$ In a subsequent study a similar platform was used to study whether basolateral polymerization of cells, a characteristic of epithelia, could be induced on single $\mathrm{MDCK}^{\mathrm{gg}}$ cells. It was unclear whether basolateral polymerization is due to increased cellcell contacts or a combined effect of morphological changes and cell-cell contacts. Both 3D SLBs in microwells as well as 2D SLBs decorated with E-cadherin showed clustered Ecadherin in the basal region, indicative of cadherin-mediated adhesions. The SLBs in microwells did not significantly alter the baso-vertical distribution of both E-cadherin and $\mathrm{Na}^{+}, \mathrm{K}^{+}$-ATPase when compared to 2D systems. Therefore in this system no evidence was found that the dimensionality of the microenvironment has an impact on E-cadherin clustering or distribution of polarity markers (Figure 1.13b). ${ }^{234}$

\footnotetext{
${ }^{\text {ee }} \mathrm{CHO}$ : Chinese hamster ovary

${ }^{\text {ff }}$ MPPC: 1-myristoyl-2-palmitoyl-sn-glycero-3-phosphocholine

${ }^{\mathrm{gg}}$ MDCK: Madin-Darby canine kidney epithelial cells 


\subsubsection{Mimicking cell-ECM contacts using peptide-modified SLBs}

Mimicking the ECM to promote neural cell adhesion onto SLBs was achieved by modifying the SLB with laminin derived peptides. Peptides derived from the extracellular matrix glycoprotein laminin-1 are known to facilitate cell adhesion and can influence neurite outgrowth. ${ }^{236}$ Among them the IKVAV motif was found to elicit these responses in PC12 ${ }^{\text {hh }}$ cells. On SLBs, it was shown that cysteine terminated IKVAV peptides conjugated with maleimido-POPE lipids promoted PC12 cell adhesion. ${ }^{168}$ AHP ${ }^{\mathrm{ii}}$ cells grown on IKVAV and scrambled VKAIV modified SLBs also show a clear preference and enhanced cell adhesion towards the natural laminin-1 epitope. At $3 \mathrm{~mol} \%$ (ca. $4.7 \mathrm{~nm}$ spacing) an 11-fold increase in cell attachment was observed for IKVAV-SLBs compared to the control VKAIV-SLBs. Utilizing the IKVAV motif on mobile SLBs and poly-electrolyte-multilayers (PEM), the effect of ligand density and mobility was explored on AHP cells. In both cases a clear trend towards more cell attachment was observed for higher ligand densities, following a sigmoidal dependence on peptide concentration. Remarkably, early cell attachment within 1 hour, was not affected by the lateral mobility of the ligands in the SLB. ${ }^{237}$ To gain more insights into AHP cell behaviour over longer periods of time, mobile SLBs were modified in situ with varying densities of the IKVAV peptide ranging from 0-10 mol \% (0-ca. $2.5 \mathrm{~nm}$ spacing) (Figure 1.14a). Compared to control polynithine/laminin surfaces, IKVAV modified SLBs at an optimal density of 3 mol\% (ca. $4.7 \mathrm{~nm}$ spacing) displayed similar cell adhesion kinetics after 1 hour of cell culture. Interestingly, after 8 days of culture distinct cell clusters were observed for AHP cells grown on IKVAV-SLBs while monolayer formation was observed for the control polynithine/laminin surfaces. Further examination of AHP cell differentiation, e.g. GFAP (glia) and MAP2ab (neurons) did not show significant differences. Various explanations exist to explain the appearance of cell clusters, such as the absence of other adhesive ligands found in laminin, recruitment of mobile IKVAV attachment points thereby resulting in depletion, removal of the peptide-lipid from the SLB and also neighbouring cells could provide a more stable anchoring point and would therefore be more preferred than the SLB. ${ }^{238}$

More recently, NSC ${ }^{\mathrm{jj}}$ cell adhesion was explored on ECM mimicking SLBs (Figure 1.14b). RGD peptide lipids were synthesized and incorporated in mobile SLBs. Upon introduction of the RGD-modified lipids up-regulation in cell attachment was observed for both RGD derivatives that were studied e.g. bsp-RGD(15) and GRGDSP. The longer bsp-RGD(15) sequence, i.e. GGGNGEPRGDTYRAY, is assumed to be present in a looped conformation stabilized by the opposite-charge interactions between glutamic acid and arginine providing improved integrin receptor engagement compared to GRGDSP. In addition,

\footnotetext{
${ }^{\text {hh }}$ PC12: cell line derived from a pheochromocytoma of the rat adrenal medulla

ii AHP: adult hippocampal progenitor cells

${ }^{\mathrm{jj}} \mathrm{NSC}$ : adult hippocampal neural stem cells
} 
surface tethering was shown to induce a type II $\beta$-turn, found in the more potent cyclic RGD derivatives. Cell morphology in the case of bsp-RGD(15), resembled observations on control laminin surfaces while more spheroid cells and cell clusters were observed on GRGDSP presenting SLBs, suggesting improved integrin engagement of bsp-RGD(15) over GRGDSP-modified lipids. ${ }^{239}$ Due to the increased potency of cyclic RGD, the use of such ligands to modify mobile SLBs was investigated. To this end, a multivalent CRGD ligand was evaluated on HEK293 ${ }^{\mathrm{kk}}$ cells. The ligand bears four CRGD motifs ( $\varnothing 3 \mathrm{~nm}$ ) and is able to bind one $\alpha_{v} \beta_{3}$ integrin receptor $(\varnothing 10 \mathrm{~nm}$ ). Cells cultured at densities of $1 \mathrm{~mol} \%$ (ca. $10 \mathrm{~nm}$ spacing) were able to flatten and form filopodial extensions. In addition, these cells were able to migrate over the SLB surface. At lower densities of the peptide ligands, cells were not able to flatten and instead the cells adopted a spheroid morphology. ${ }^{240}$

The effect of ECM-ligand density in combination with varying the lateral mobility was explored for human primary chondrocytes as described in detail in Chapter 5. The experiment revealed distinct cell behaviour upon increase of a RGD amphiphile. In the case of liquid-state SLBs for the mobile presentation of RGD, cell viability and density scaled with an increase in RGD doping density. At an interligand spacing of $<100 \mathrm{~nm}$ cell spreading was noted as was revealed by cell shape analysis. On the contrary, gel-state SLBs doped with a relevant amount of immobile RGD amphiphiles showed an overall high cell viability and spreading with a tendency to form cell aggregates. The onset of aggregate formation was observed for interligand spacings of $100 \mathrm{~nm}$ and lower. The variation in lateral mobility was correlated to differences in focal adhesion assembly and cytoskeleton organization, most likely caused by differences in traction forces. ${ }^{25}$

\subsubsection{Mimicking cell-ECM contacts by decorating SLBs with ECM proteins}

To more carefully mimic the cell-ECM interface native ECM molecules can also be incorporated in SLBs (Figure 1.14c). Although tissue culture plastics can be coated with ECM molecules, they do not offer control over the density and the mobility and are known to be fouling. In the case of SLBs, ECM molecules are coupled directly to lipids embedded within the SLB, e.g. type I collagen, which can associate to integrin receptors, can be linked via its lysine residues to NHS-activated DP-NGPE lipids in inert, zwitterionic POPC SLBs. During the reaction the increase in mass was monitored using QCM-D and the amount of collagen was varied as a function of DP-NGPE doping densities ranging from 0 to $40 \mathrm{~mol} \%$. At DP-NGPE densities over $40 \%$ an incomplete bilayer formation was observed. Upon AFM analysis micrometer sized collagen fibrils were observed on SLBs bearing 20 mol\% DPNGPE while the thickness of the bilayer was confirmed. In addition, the higher the amounts of collagen on the bilayer, the lower the lateral diffusion on the SLB observed.

\footnotetext{
${ }^{\mathrm{kk}}$ HEK293: human embryonic kidney cells 


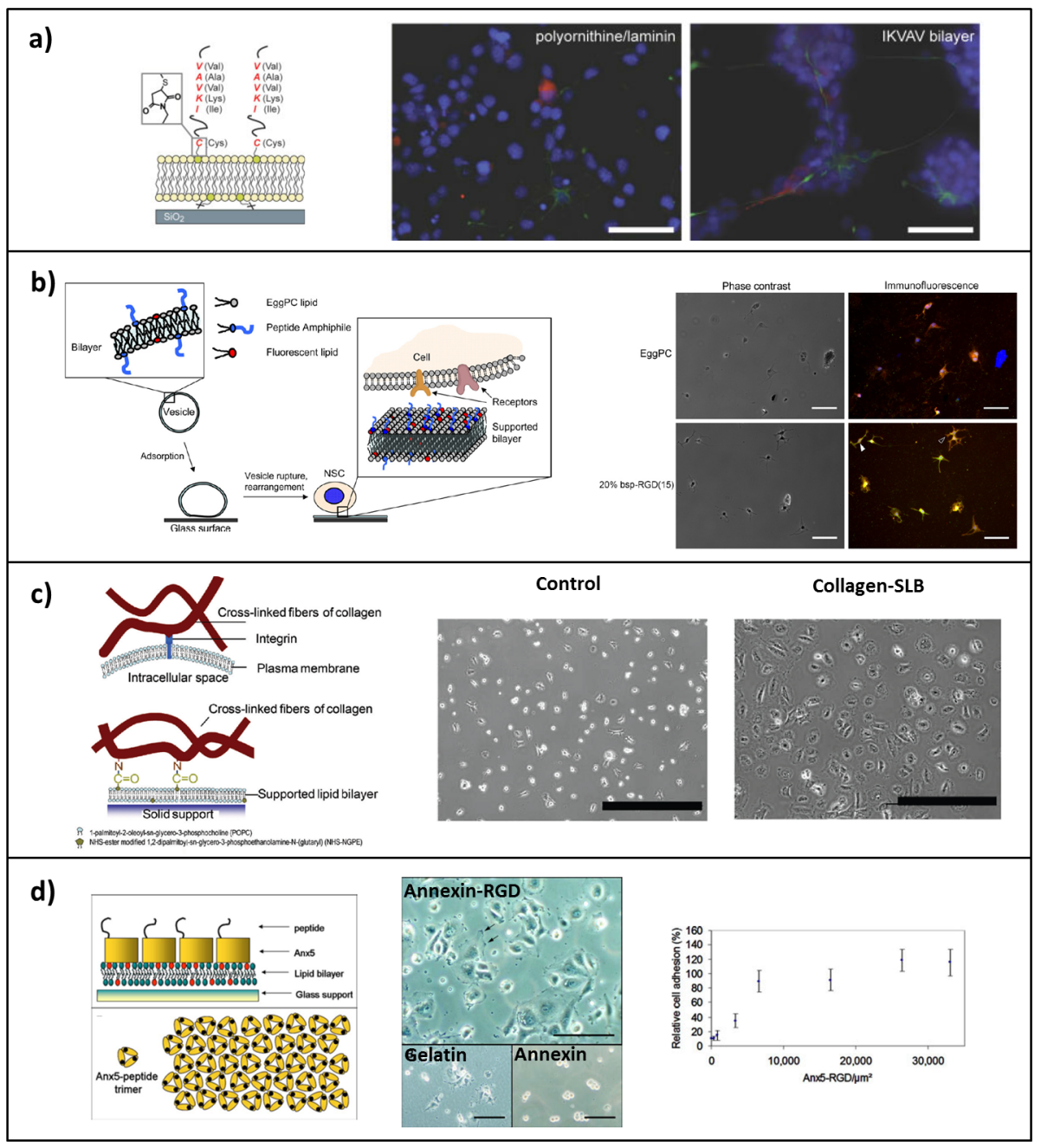

Figure 1.14: Mimicking cell-ECM contacts on SLBs; a) SLBs were modified with laminin-derived IKVAV peptides to support AHP cell adhesion. Reprinted with permission. ${ }^{238}$ Copyright 2008 , John Wiley \& Sons. b) Fibronectin-derived RGD peptide amphiphiles were used to modify SLBs for NSC adhesion. Reprinted with permission. ${ }^{239}$ Copyright 2010, Elsevier. c) Direct modification of the SLB with ECM proteins was shown for collagen. Collagen-modified SLBs supported cell adhesion and spreading. Reprinted with permission. ${ }^{170}$ Copyright 2010, American Chemical Society. d) The use of a 2D Anx5 protein network for the presentation RGD peptides. Cell adhesion behaviour could be influenced by altering the RGD surface density on the $2 \mathrm{D}$ protein crystal. Reprinted with permission. ${ }^{241}$ Copyright 2007, American Institute of Physics. 
These changes are most likely attributed to the increasing amount of saturated DP-NGPE, which has a higher $\mathrm{T}_{\mathrm{m}}$ compared to POPC, $41^{\circ} \mathrm{C}$ vs. $-10^{\circ} \mathrm{C}$. Although no evidence of phase separation was found, the presence of nanoscopic domains of DP-NGPE could act as diffusion barriers thereby retarding the lateral mobility. A10" cells were seeded on the collagen decorated SLBs and the growth of A10 cells was evaluated. On collagen modified SLBs a 2.5 -fold increase in cell number was observed as well as a 6-fold increase in projected cell area when compared to bare POPC SLBs after overnight cell culture. ${ }^{170}$ Adopting the same chemistry, various proteins were immobilized on mobile SLBs and positively evaluated for NIH3T3 cell growth. Next to collagen type I, fibronectin or BSA, and combinations of two proteins on SLBs were evaluated in cell culture. A direct comparison was made to tissue culture polystyrene (TCPS). ${ }^{242}$ The first protein was covalently attached to the surface using NHS chemistry while the second protein was adsorbed on the first layer. In the case of covalently attached collagen type I and adsorbed fibronectin, specific binding between collagen type I and fibronectin binding domains was suggested based on comparative QCM-D studies with BSA. ${ }^{243}$ Upon cell analysis after 6 hours of cell culture, preferential adhesion was noted to the collagen type I and fibronectin modified SLBs. Cell adhesion was further improved on bimodal surfaces displaying covalently attached collagen type I and adsorbed fibronectin, which was reflected in both cell number and area. It was found that NIH3T3 fibroblasts were able to actively remodel their fibronectin environment on TCPS modified surfaces while this behaviour was not observed in the case cells were grown on ECM-presenting SLBs. This can be ascribed to the inherent non-fouling nature of the PC SLBs that were used. Nevertheless, strong preferential adhesion towards ECM-modified SLBs was observed. ${ }^{242}$

In an attempt to generate a mimetic cell adhesive surface based on polysaccharides, hyaluronic acid (HA) was conjugated to an SLB surface. HA is an anionic linear polysaccharide consisting of disaccharide repeats bearing a carboxyl group. HA may act as an attractive or repulsive spacer between cells depending on the presence of HAreceptors e.g. CD44. Early work where HA was conjugated to glass surfaces, lacked control over the HA density and mobility. ${ }^{244}$ To overcome these shortcomings, recombinant Histagged p32 HA binding protein was chelated to the SLB surface via chelating lipids (DOGSNTA). Subsequent introduction of HA showed specific binding to the p32-modified SLB. ${ }^{245}$ More recently, HA grafted SLBs were prepared using biotinylated lipids and HA. ${ }^{246}$ Similarly glycosaminoglycans (GAG) and sulphated GAGs like chondroitin sulphate (CS), large unbranched polysaccharides found in the ECM, were conjugated to SLBs as well. CS plays an important role in bone and cartilage tissue and is capable of binding growth factors and ECM molecules like bone morphogenic protein 2 (BMP-2) and collagen type I,

\footnotetext{
"A10: vascular smooth muscle cells 
respectively. CS was covalently linked to DOPE-COOH ${ }^{\mathrm{mm}}$ or DOPE- $\mathrm{NH}_{2}$ bearing SLBs. In both cases successful modification was confirmed using QCM-D. Studying the $\Delta D / \Delta f$ ratio similar viscoelastic properties for both modification strategies were found as well as preservation of lateral mobility, ca. $0.9 \mu \mathrm{m}^{2} / \mathrm{s}$. Bio-activity of the layers was confirmed with collagen type I and BMP-2 binding. ${ }^{247}$

\subsubsection{Mimicking cell-ECM contacts using a protein network for peptide presentation} Instead of covalent attachment of ECM molecules or peptide sequences to the SLB directly or the use of peptide amphiphiles, the use of an intermediate rigid 2D protein layer as a carrier for ligand presentation was shown (Figure 1.14d). Here, Anx5 was used to provide a self-assembling 2D matrix for ECM peptide presentation. ${ }^{224}$ These highly ordered structures comprise of 33,000 molecules $/ \mu \mathrm{m}^{2}$ and have a spacing of $6 \mathrm{~nm}$ between adjacent Anx5. Mutant rat Anx5 was prepared of which the single cysteine residue at position C314S was removed and a single cysteine residue was introduced at position T163C. These cysteine bearing mutants (Anx5-SH) allowed for disulphide bond formation between cysteine containing RGD (GCRGYGRGDSPG) or IKVAV (CSRARKQAASIKVAVSADR) peptides. Early work on Anx5 showed 2D crystal formation on DOPS doped SLBs. ${ }^{224}$ Hence, DOPC and DOPS (7:3 w/w) SLBs were prepared to allow for Anx5 2D crystal formation. QCM-D experiments confirmed calcium-dependent binding of Anx5-RGD and Anx5-IKVAV derivatives to the SLB surface. The higher dissipation values indicate a highly hydrated, extended structure, favourable for integrin interaction. AFM and TEM analysis further confirmed that 2D crystal formation was not impaired in case of Anx5-peptides. Similar to native Anx5, a hexagonal packed crystal structure was observed. TEM derived unit cell dimension and the $p 6$ symmetry are similar to Anx5. Integrin engagement was studied with $\mathrm{HSVE}^{\mathrm{nn}}$ and undifferentiated $\mathrm{MES}^{\circ \mathrm{O}}$ cells. HSVE cells were able to adhere on Anx5RGD SLBs showing cell spreading and focal contact points on the cell periphery, similar to HSVE cells grown on gelation controls. Native Anx5 SLBs did not promote HSVE cell adhesion and spreading while Anx5-IKVAV only showed limited cell adhesion. In this case, quiescent cells displayed a spheroid morphology. Cell density observed on Anx5-RGD was higher than the gelatin control surfaces. MES cells however, displayed similar growth behaviour on Anx5-IKVAV and gelatin surfaces, often used as a reference substrate. This effect was less pronounced on Anx5-RGD surfaces. Dilution of peptide bearing Anx5 was achieved by addition of native Anx5, providing means to alter the interligand spacing, proven by a liposome binding assays. For both Anx5-RGD and Anx5-IKVAV, cell adhesion was observed as low as 300 peptides/ $\mu \mathrm{m}^{2}$ corresponding to $58 \mathrm{~nm}$ spacing, for HSVE and MES cells respectively. ${ }^{241}$ These values are similar to values obtained by Spatz and co-

\footnotetext{
${ }^{m m}$ DOPE: 1,2-dioleoyl-sn-glycero-3-phosphoethanolamine

${ }^{n n}$ HSVE: human saphenous vein endothelial cells

${ }^{\circ}$ MES: mouse embryonic stem cells
} 
workers. ${ }^{248}$ Recently, the stabilizing effect of Anx5 was shown on pore spanning SLBs. AFM indentation studies showed increased rigidity of the Anx5 decorated SLB. ${ }^{249}$

\subsubsection{Cell adhesion on charged SLBs}

In contrast with other studies that focussed on mimicking cell-cell or cell-ECM contacts on SLB surfaces, the use of charged SLBs can be used as a platform for cell adhesion. The incorporation of simply charged species allows differentiation between the extent of cell adhesion. This was demonstrated with the addition of DOTAP ${ }^{\mathrm{pp}}$, a positively charged lipid that was added in zwitterionic PC SLBs. Cultivation of rat embryonic cortical neurons showed an increase in cell adhesion on the positively charged SLB (Figure 1.15a). Moreover, they found a positive correlation between doping density of DOTAP and cell viability. Cell adhesion was attributed to the attractive forces between the positively charged SLB and negatively charged neuronal cell membrane. Protein deposition can however not be excluded to have inferred in this process. In addition, the stability of the SLB for at least 19 days was demonstrated under neuronal cell culture conditions. ${ }^{250}$ Defects in the SLBs could be observed that corroborate with previous observation by Tamm and McConnell. ${ }^{32}$ A similar effect was observed previously with Hela ${ }^{\text {q9 }}$ and NIH3T3 cells cultured on SLBs of varying compositions. Alterations were made in charge densities by addition of either positively or negatively charged lipid within zwitterionic PC SLBs. ${ }^{251}$ For example, DAP ${ }^{\text {rr }}$ and PS were used as a positively and negatively charged lipid, respectively. Their findings with these naturally adherent cells showed that the negative charge on PS was able to support cell adhesion at 5 mol\% doping density. Strikingly, similarly charged PG lipids did not show this behaviour demonstrating the chemical specificity of initial cell attachment in these cell lines and the potential of directing cell fate by charge only.

\subsection{Use of gradient SLBs for cell studies}

\subsubsection{Screening of RGD-lipid density in SLBs}

Screening of biological probes on cell function is of great interest to the scientific community. A gradient allows for screening variations in surface compositions against one cell population in one experiment. Using RGD-modified lipids and a microfluidic network device, changes in RGD doping density were achieved on mobile SLBs prepared on a patterned surface (Figure 1.15b). Mouse fibroblasts were grown on these SLBs with or without a hydrophilic spacer consisting of poly-ethylene-oxide (PEO). Only the RGD amphiphiles that bear a hydrophilic spacer were able to promote cell adhesion in a

\footnotetext{
${ }^{p p}$ DOTAP: 1,2-dioleoyl-3-trimethylammonium-propane

${ }^{\text {qq }}$ HELA: human cervical carcinoma cells

${ }^{r r}$ DAP: 1,2-dipalmitoyl-3-dimethylammonium-propane 46
} 


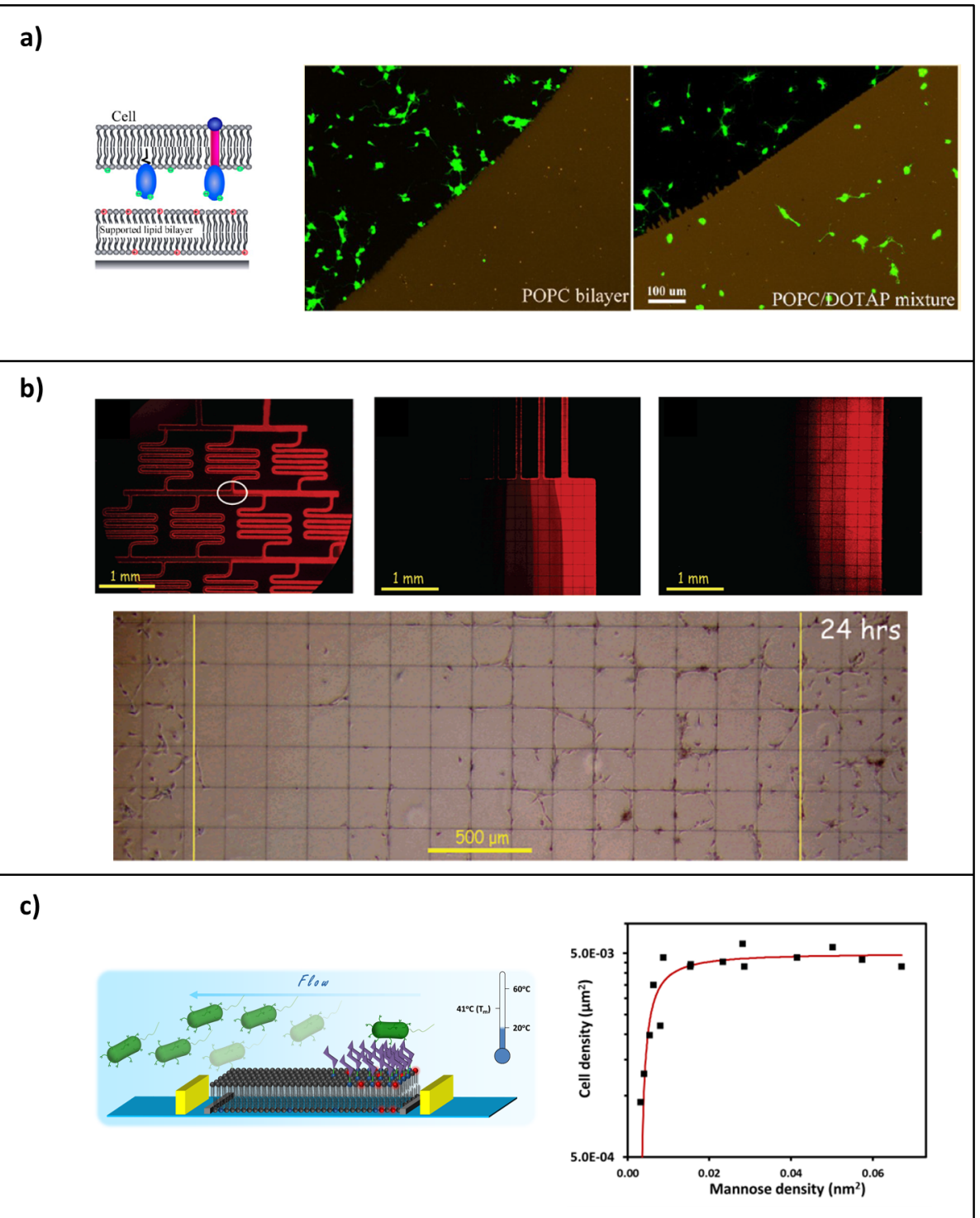

Figure 1.15: Charged and gradient SLB cell culture platforms; a) the use of positively charged SLBs in neuronal cell culture was evaluated. Adhesion is a cumulative effect of protein deposition and electrostatic attraction between the anionic cell membrane and cationic SLB. Reprinted with permission. ${ }^{250}$ Copyright 2012, American Chemical Society. b) Using an intricate fluidic network over a patterned surface and flowing lipid vesicles with varying densities of RGD amphiphile, RGD surface density could be tuned in SLBs as confirmed with cell adhesion studies. Reprinted with permission. ${ }^{252}$ Copyright 2007, American Chemical Society. c) The use of locked-in gradients of mannose in SLBs were used to study the binding of E. coli (ORN 178) under varying shear stress conditions. 
concentration-dependant manner due to enhanced integrin engagement. A plateau was reached at higher RGD densities with respect to the fraction of adhered cells while an increasing trend in cell area was observed. ${ }^{252}$

\subsubsection{Use of locked-in SLB gradients for cell studies}

Studying cell-cell recognition events on artificial surfaces often require multiple receptorligand complexes to form as they do natively. ${ }^{253}$ For instance, microbial pathogens display copies of receptors on their cell surfaces that bind to their respective target ligands on their hosts. ${ }^{254}$ In the case of surface immobilization, the surface density of these ligands was found to influence this behaviour. To study these phenomena in greater detail the field of quantitative glycomics have made use of glycan microarrays to identify and characterize pathogens that target glycoproteins on cell surfaces. ${ }^{255}$ Although such analysis has been successful in determining the specificity and affinity of pathogenic lectins towards carbohydrates, the effect of glycan surface density has been a more difficult parameter to investigate. ${ }^{256-258}$ Such methods often require the preparation of several surfaces with discrete ligand densities, a cumbersome process prone to errors. Such discrete ligand densities can be relatively easily introduced in SLBs. This has been demonstrated for discrete SLB surfaces that are modified with varying densities of mannose. ${ }^{259}$ Mannose, present on mammalian cell surface glycoproteins, can be bound by fimbrial FimH receptors found on pathogenic $E$. coli strains. Although specific immobilization was observed in these mimetic SLB systems, analysis is limited to the concentrations that were prepared a priori. Moreover, mannose-FimH interaction not only depends on the surface density of mannose, ${ }^{259}$ but is affected by shear stress due to flow conditions, ${ }^{260}$ which can normally be assessed using separate techniques. Stable and continuous surface gradients were prepared in SLBs of mannose and used to study the binding of E. coli ORN 178 (Chapter 4) (Figure 1.15c). The proposed method allowed for the analysis of binding in relation to the applied shear stress induced by bulk flow provided by microfluidics. This one-chip glycomics analysis provided insights into FimHmannose binding at varying surface densities and levels of shear stress while using a membrane mimetic system.

\subsection{Use of patterned SLBs in cell studies}

\subsubsection{Use of micro-contact printed patterned SLBS}

The versatility of SLBs is also reflected by the ease to prepare surface patterns or varying compositions, vide supra. Physical diffusion barriers given by the microcontact printed lines of fibronectin proteins are employed to confine the lateral mobility of fluorescently labelled lipids in mobile SLBs (Figure 1.16a). ${ }^{68}$ These fibronectin patches embedded within cell repellent zwitterionic SLBs are used to study the effect of geometry and dimensions 
onto the adhesion of $\mathrm{CPAE}^{\mathrm{SS}}$ cells. The results showed that adhesion occurred exclusively in the fibronectin regions. In addition, cell spreading behaviour and extent could be modulated by varying the size of printed square regions. ${ }^{69}$ Similarly, fibronectin patterns were used to provide stable anchoring points to MCF-7 cells while still presenting cells with mobile ligands, such as human (h)EGF. A patterned array of $5 \mu \mathrm{m}$ circular spots spaced $10 \mu \mathrm{m}$ apart were used. MCF-7 cells engaged multiple fibronectin spots resulting in cell flattening and spreading, not observed with MCF-7 cells grown on mobile hEFG-SLBs (Figure 1.16b). The use of a fibronectin array increased hEFG-associated fluorescence clustered by MCF-7 cells by 1.5 to 2 -fold over hEFG doped SLBs only. ${ }^{225}$

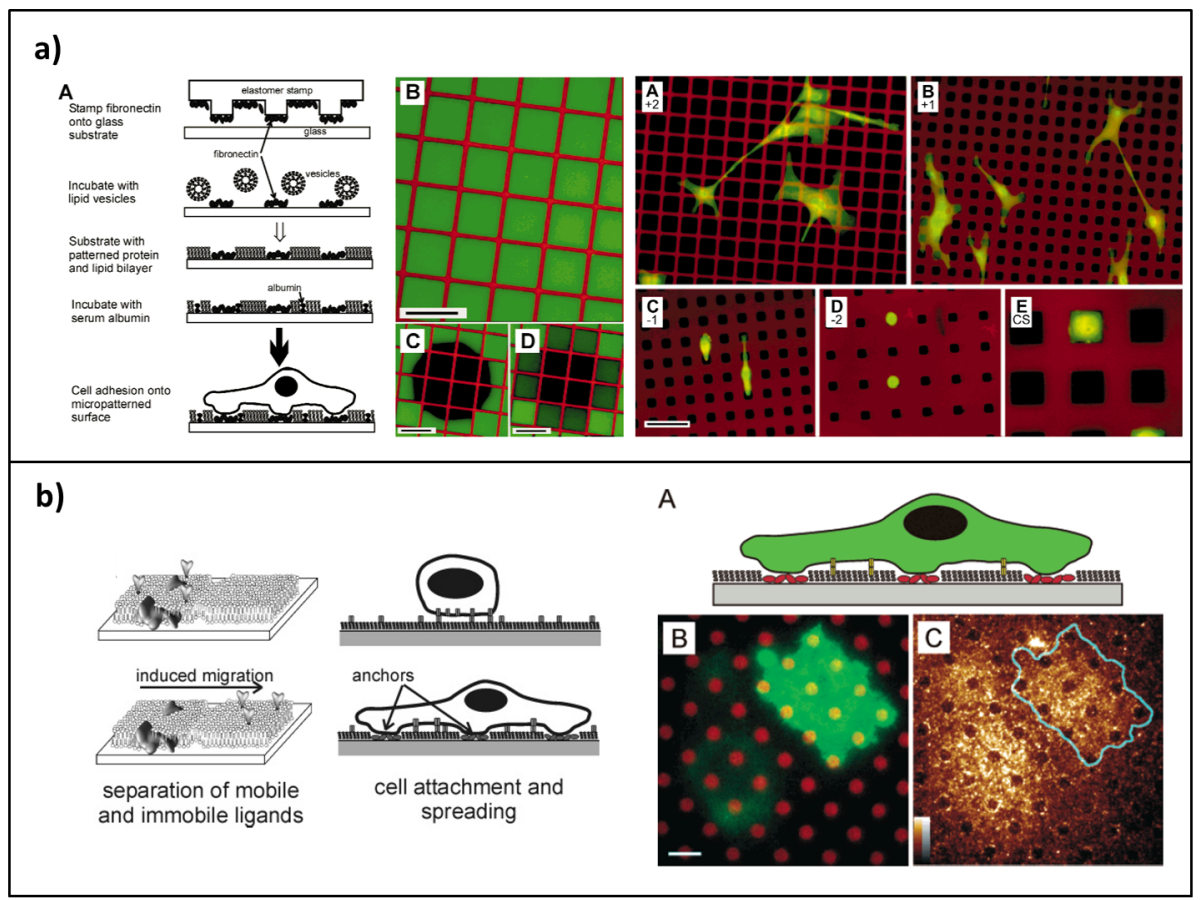

Figure 1.16: Micro-contact printing for generating patterned SLBs for use in cell studies; a) printed barriers of fibronectin served as anchoring points for cell adhesion while the SLB acted as a nonfouling background in the exposed regions of the substrate. Reprinted with permission. ${ }^{69}$ Copyright 2001, John Wiley \& Sons. b) Study of mobile hEFG associated with the SLB and cell adhesion. Cell spreading and flattening was only observed in cases where fibronectin spots were printed on the substrate. Reprinted with permission. ${ }^{225}$ Copyright 2005, American Chemical Society.

\footnotetext{
${ }^{\text {ss }}$ CPAE: cow pulmonary arterial endothelial cells
} 


\subsubsection{Use of particle-based SLB systems}

To study the effect of surface rigidity on the cytoskeletal dynamics upon cell adhesion, mobile and immobile presentation of E-cadherin can be employed. Instead of presenting E-cadherin on a planar SLB surface as described in the previous section, the molecule was delivered on silica beads measuring $5 \mu \mathrm{m}$ in size. These beads were modified with a NTAbearing mobile PC SLB to allow the chelation of hEFG to occur. Other beads were incubated with a hEFG solution to physisorb the protein. The resulting beads display therefore ligands in a mobile or immobile fashion, respectively (Figure 1.17a). Mobility of the ligand was found to be $0.3 \mu \mathrm{m}^{2} / \mathrm{s}$ with an $>80 \%$ mobile fraction. In this study, the human analogue of E-cadherin was used since the extracellular domain of canine Ecadherin was found to be essentially immobile on SLBs. This human analogue displayed cross-species activation and could therefore be used instead of canine E-cadherin with MDCK cells. These MDCK cells were grown on collagen coated coverslips and transfected for a GFP-tagged Rac1 and DsRed-E-cadherin. Rac1 recruitment, member of the Rho family small GTPases, is heavily involved as an E-cadherin downstream and upstream effector of cytoskeletal dynamics, was shown for mobile hEFG presentation. This was evidenced by an increased recruitment of Rac1 compared to the immobile controls. The amount of adsorbed and immobile hEFG was around 300 molecules $/ \mu \mathrm{m}^{2}$. However, a four times higher concentration (ca. 1200 molecules $/ \mu \mathrm{m}^{2}$ ) of adsorbed, immobile hEFG showed similar Rac1 recruitment to mobile hEGF presentation at low densities. No evidence of Rac1 recruitment was found for bare SLB control and low concentration immobile hEFG. ${ }^{261}$

More recently, an adaptation of block copolymer micelle nanolithography (BCML) developed by Spatz and co-workers ${ }^{262}$ was combined with SLBs (Figure 1.17b). BCML enables the preparation of defined arrays of gold nanoparticles with a distinct quasihexagonal spacing. ${ }^{263}$ Nucleation sites for nanoparticle growth are created during the phase separation of the block copolymers into a micelle array. After removal of the organic components with a plasma treatment, an ordered array of gold nanoparticles is left behind on the surface of which the spacing is defined by the molecular weight of the block copolymer. In this way, gold nanoparticles of $7 \mathrm{~nm}$ in size were arrayed on glass with spacings ranging from 58 to $151 \mathrm{~nm}$ and used as a substrate for SLB pattern formation. The effect of gold nanoparticles on lipid and SLB-associated protein diffusion was studied with either a fluorescent lipid (TR-DHPE) or fluorescent protein ( $\mathrm{His}_{12}$-mGFP) chelated to NTA-DOGS lipid within mobile PC SLBs, respectively. FCS measurements revealed no effect on the lipid mobility as indicated by an unaltered TR-DHPE diffusion of $2 \mu \mathrm{m}^{2} / \mathrm{s}$, irrespective of the gold nanoparticle density. His 12 -mGFP diffusion was susceptible to particle spacing ranging from $1-2 \mu \mathrm{m}^{2} / \mathrm{s}$ with a trend towards higher mobility at lower particle densities. These results confirmed earlier work by Roiter and co-workers who demonstrated that nanoparticles measuring 1.2 to $22 \mathrm{~nm}$ in size can be embedded within 


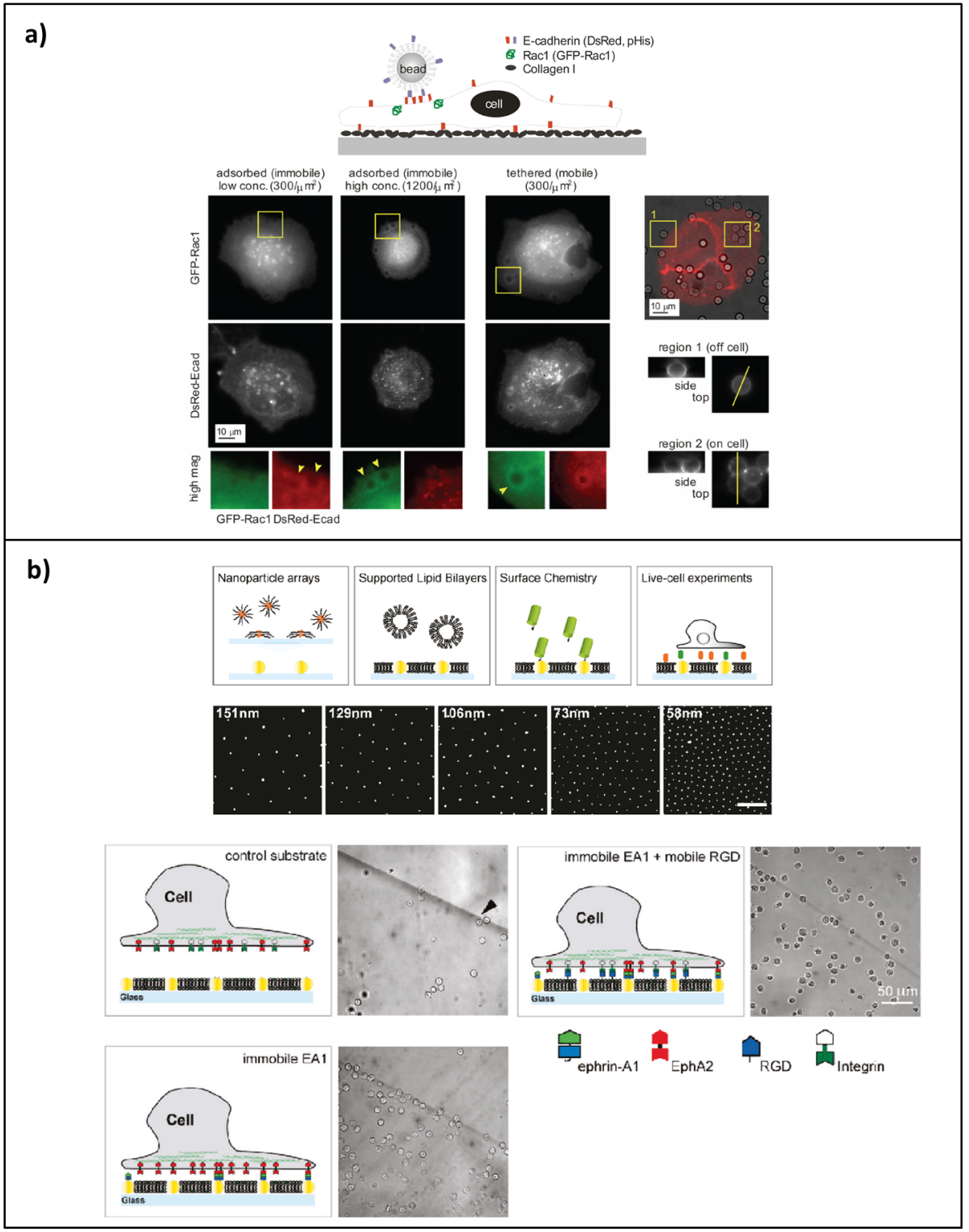

Figure 1.17: The use of particle-based SLBs for cell studies; a) silica particles either coated with physisorbed E-cadherin or presented in a mobile fashion on an SLB-coated bead were used to study Rac1 recruitment. Enhancement at low surface density was only observed when E-cadherin was presented in a mobile fashion on the SLB-coated particle. Reprinted with permission. ${ }^{261}$ Copyright 2010, Springer Science. b) Block copolymer micelle nanolithography was combined with SLBs for the study of MDA-MB-231 cell adhesion upon varying RGD and ephrin-A1 presentation. Reprinted with permission. ${ }^{263}$ Copyright 2011, American Chemical Society. 
an SLB instead of being covered by a lipid membrane. ${ }^{91,264}$ Embedded gold nanoparticles within an SLB can be used to immobilize ligands using NHS-chemistry, NTA-chelation, biotin-streptavidin interaction and in DNA hybridization. The gold nanoparticle array in combination with a mobile SLB was used to study MDA-MB- $231^{\text {tt }}$ cells. This cell line is known to overexpress the EphA2 receptor tyrosine kinase that interacts with its ligand ephrin-A1 in a juxtacrine fashion. ${ }^{265,266}$ The MDA-MB-231 cells were unable to interact with control surfaces consisting of NTA-modified SLBs and naked gold nanoparticles. Upon modification of the gold nanoparticles with ephrin-A1 ligands, artificial cell-cell contacts were formed resulting in cell adhesion to the substrate surface, but not to a control bare glass surface. Subsequent modification of the mobile SLB with RGD peptides resulted in improved cell attachment due to integrin-mediated adhesion as concluded from a more flattened and spread cell morphology, demonstrating the bimodal nature of such particlebased SLB interfaces. $^{263}$

\subsubsection{Use of metal corrals in SLB studies}

The use of SLBs prepared on glassy substrates patterned with chromium $(\mathrm{Cr})$ corrals was pioneered by Groves and co-workers to elucidate the IS formed during antigen recognition by T-cells. $^{267}$ Silica substrates patterned with $100 \mathrm{~nm}$ wide Cr-lines in various configurations that were prepared with E-beam lithography and served as a substrate for SLB formation. The bilayer was decorated with pMHCs and ICAM-1 in an attempt to mimic the native $A P C^{\mathrm{uu}}$ cell and T-cell interaction upon antigen recognition (Figure 1.18a). pMHC on the APC membrane interacts with the T-cell receptor (TCR) to form a region that can be assigned as the central supramolecular activation cluster (c-SMAC). This region is surrounded by a ring of interactions between leukocyte function associated antigen-1 (LFA-1) on the T cell membrane and ICAM-1 on the APC that can be assigned as the peripheral supramolecular activation cluster ( $p$-SMAC). Upon T-cell culture on the APCmimetic and patterned surfaces, stable formation of TCR microclusters were observed and stable trapping of these clusters by the Cr-barriers. In general, these microclusters were pulled into the corner of the grid that are nearest to the centre of the IS. Signalling activity was evaluated by the cytoplasmic distribution of phosphorylated tyrosine (pY) residues associated with TCR clusters. It was found that the frustrated IS imposed by the surface patterns retained high specific $p Y$ levels. However, microclusters towards the centre of the IS showed a loss in activity similar to IS formed on unpatterned APC-mimetic SLBs. These results suggest that the duration of TCR-pY signalling is related to the radial position of the TCR instead of cluster size. This was confirmed with the flux of intracellular $\mathrm{Ca}^{2+}$ induced by TCR antigen recognition. ${ }^{267}$ In a subsequent study using a similar platform, the effect of LFA-1 clustering on protein sorting in the IS was explored (Figure $1.18 \mathrm{~b}$ ). ${ }^{268}$ This was

\footnotetext{
${ }^{t t}$ MDA-MB-231: highly invasive human breast cancer cell line

uu APC: antigen presenting cell 


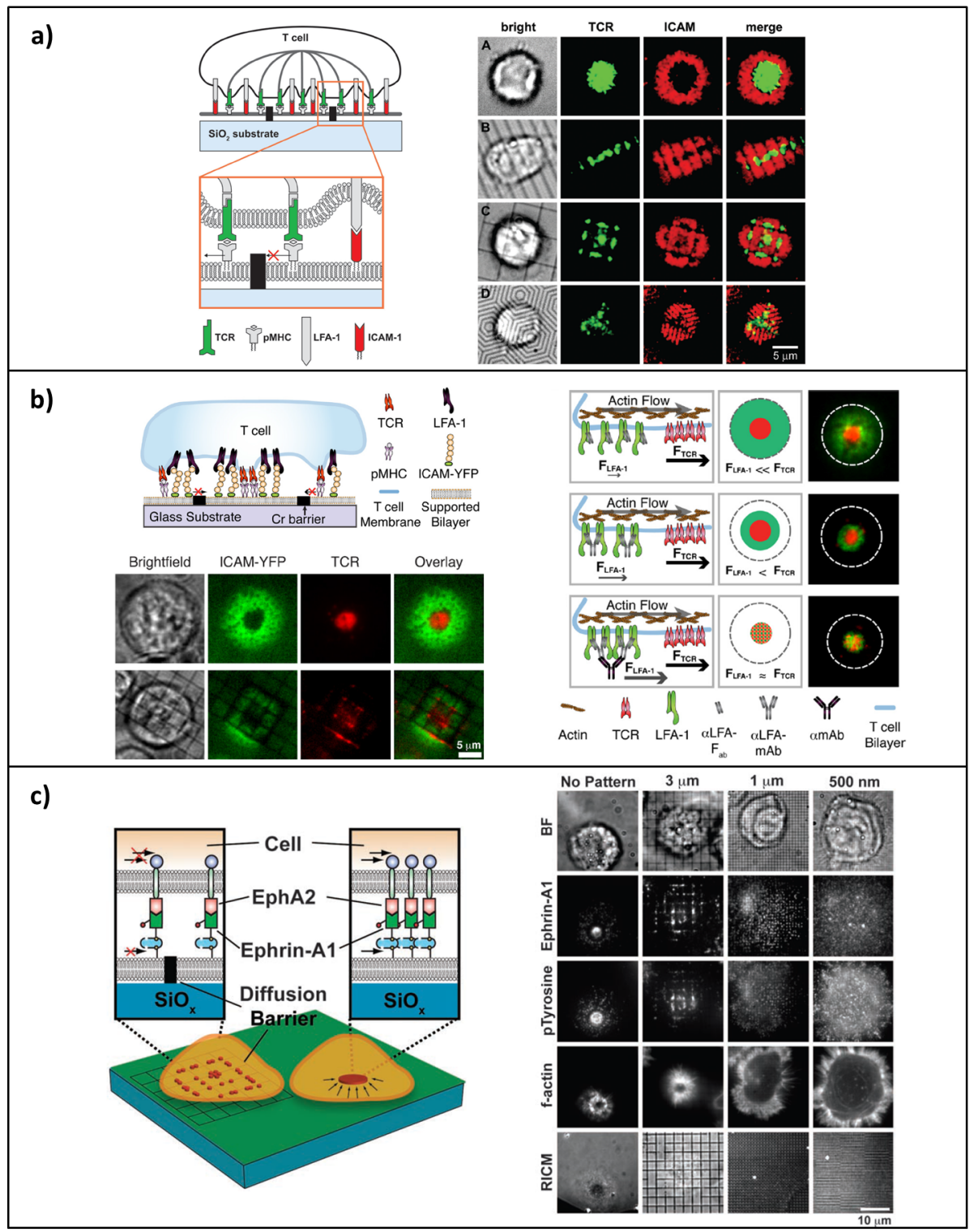

Figure 1.18: Use of metal corrals in SLBs for cell studies; a) analysis of IS formation on planar SLBS and SLBs with metal corrals. Reprinted with permission. ${ }^{267}$ Copyright 2005, American Association for the Advancement of Science. b) Protein sorting in the IS by crosslinking LFA-1 by means of antibodies. Reprinted with permission. ${ }^{268}$ Copyright 2009, United States National Academy of Sciences. c) Ligand-induced activation and the effect of receptor clustering using SLBs with corrals. Reprinted with permission. ${ }^{265}$ Copyright 2010, American Association for the Advancement of Science. 
achieved by either direct antibody crosslinking of the receptor or crosslinking of the ICAM1 on the SLBs. Upon clustering of LFA-1 in a bivalently or tetravalently fashion, the distribution could be biased towards the centre of the IS. Similar effects were shown for bivalent crosslinking of ICAM-1 on the SLB. As a result, protein sorting in the IS is dependent on protein cluster size and thus frictional coupling of the actin cytoskeleton. ${ }^{268}$ Moreover, it was found that T-cell triggering was dependant on the amount of activating ligand per TCR cluster and not on the total amount of ligand presented to the cell. This effect was studied by varying the size of the $\mathrm{Cr}$-corrals thus laterally limiting the amount of SLB bound ligand to interact with TCR clusters. ${ }^{269}$ These patterned metal corrals can be combined with microfluidics to fabricate patterned SLBs. For example, an interdigitated array of photoresist on glass was combined with a microfluidic array that could provide three laminar flows used for vesicles. By adopting this approach, the interdigitated region could present two distinct bilayer compositions. These alternating $5 \mu \mathrm{m}$ wide regions were used to study spatially segregated, micro-patterned ligands to cells. The TCR and LFA-1 were studied on APC. The two SLBs, separated by the photoresist barrier, were doped with either biotinylated or NTA head-group modified lipids. After streptavidin was associated to the biotinylated SLB, biotinylated-OKT3 antibody was allowed to interact with streptavidin. The OKT3 antibody is known to recognize and activate the TCR receptor on the APC cell membrane. A His-tagged derivative of the natural ligand of LFA-1, ICAM-1, was chelated to the NTA-modified SLB. Analysis of both complexes revealed a lateral mobility of 0.39 and $0.23 \mu \mathrm{m}^{2} / \mathrm{s}$ for ICAM-1 and OKT3, respectively. After incubating the cells for 2 hours clustering of both complexes was observed. These complexes tended to be localized towards the centre of the APC cell. The spatial reorganization suggest IS formation requires concurrent presentation of both OKT3 and ICAM-1, to achieve the traditional 'bull's eye' formation where TCR localizes in the centre and LFA-1 on the periphery. $^{270}$

Using SLBs with Cr-corrals, large scale mobility of the EphA2 receptor tyrosine kinase could be modulated thereby altering cell behaviour (Figure 1.18c). ${ }^{265}$ The EphA2 is implicated and functionally altered in a various types cancers. For instance, in human breast cancer the receptor is overexpressed in $40 \%$ of the cases. ${ }^{271}$ Traditionally, EphA2 is activated by its natural ligand Ephrin-A1, resulting in receptor dimerization and triggering subsequent signalling pathways. In vitro, EphA2 activation is only achieved with soluble Ephrin-A1 that has been chemically cross-linked. Here, cross-linked Ephrin-A1 modified SLB were used to study the downstream effects of freely 2D laterally mobile ligands and space constrained ones. Cross-linked Ephrin-A1 was required since monovalent, freely soluble ephrin-A1 ligand binds to EphA2 but fails to trigger activation in solution. ${ }^{272}$ To this end, the glass substrates with $\mathrm{Cr}$-barriers of varying sizes were back-filled with mobile SLBs decorated with Ephrin-A1. This was achieved by doping PC SLB with $0.1 \mathrm{~mol} \%$ of biotinylated lipid 
and associating ligands via biotin-streptavidin interaction. Care was taken to ensure similar ligand densities on the SLBs compared to the density of EphA2 receptors on natural membranes, i.e. 800 molecules/ $\mu \mathrm{m}^{2}$. Upon MDA-MB-231 cell culture on unconstrained SLBs, clusters of EphA2 were observed displaying inward radial transport on Ephrin-A1 modified, mobile SLBs. Co-movement with $\beta$-actin suggested EphA2 transport governed by actomyosin contractility, confirmed by means of Rho kinase inhibition. This long-range organization effect was diminished when the mobile SLB was exchanged for immobile DPPC-based SLBs, confirmed by decreased phosphorylation of the receptor. To rule out intermembrane anchoring, RGD peptide was displayed alongside the Ephrin-A1 ligand in varying molar ratios. Irrespective of RGD:Ephrin-A1 molar ratio, EphA2 receptor translocation was observed on mobile SLBs. Varying the degree of partitioning of the Ephrin-A1 receptor in the mobile SLB by means of a patterned $\mathrm{Cr}$-grid, two distinct states were identified. Small $500 \mathrm{~nm}$ girds allowed cells to spread and showing predominantly peripheral f-actin and lamellipodia, similar to cells cultured on RGD modified glass without Ephrin-A1. Here, the physical resistance to EphA2 receptor transport altered the threshold for Ephrin-A1 mediated cytoskeletal reorganization and ADAM10 recruitment, involved in ectodomain transshedding of Ephrin-A1 as a result of EphA2 ligand binding. ${ }^{273}$ The results of this study demonstrate that physical interference with receptor clustering can alter the ligand-induced activation. ${ }^{265}$

In a subsequent study, successful activation of EphA2-expressing cells was shown for monovalent Ephrin-A1 chelated to the SLB. ${ }^{266}$ A monomeric fusion protein was immobilized to the bilayer by means of Ni-NTA chelation. Experiments showed stable anchoring of the complex for at least 16 hours. Specific immobilization was confirmed by the addition of $100 \mathrm{mM}$ EDTA. Using FCS and photon arrival times (PCH) showed that the membrane-associated Ephrin-A1 is predominantly monomeric and laterally mobile, $2.3 \pm$ $0.2 \mu \mathrm{m}^{2} / \mathrm{s}$. Similar observations were made with respect to mechanical constraints imposed by surface patterns in the case of monomeric Ephrin- $\mathrm{A} 1^{266}$ when compared to observations with cross-linked derivatives. ${ }^{265}$ The results illustrate that the spatiomechanical sensitivity is not an artifact of the chemical cross-linking of the ligand, and that the monomeric ligand allows for investigation of initial EphA2-Ephrin-A2 clustering and transport on SLBs. ${ }^{266}$

Fabricated Cr-line patterned glass surfaces were functionalized with RGD on mobile SLBs via biotin-streptavidin interactions. In a variety of adherent cell lines, HeLa and MDCK, a trend towards a more spread morphology was observed as a function of decreasing pitch of $\mathrm{Cr}$-lines. Interestingly, RGD-integrin clusters that were formed after initial cell contact with the SLBs tended to move laterally towards each other. When line patterns were introduced these clusters piled up against the metallic barriers. Cell spreading could be 
modulated by the density of periodic metallic line barriers. Thereby impeding the lateral mobility and defining where forces are passively generated. Contractility of the actin and myosin rich RGD-integrin clusters could effectively be blocked by myosin-II inhibitor blebbistatin. The results suggest that early integrin activation and cluster formation, comprising of talin, paxillin and FAK, are independent of lateral forces, although vinculin recruitment was only observed upon contraction on Cr-line surfaces. ${ }^{274}$

Since force generation can be explored using SLBs and Cr-diffusion barriers, ${ }^{24,265,274}$ matrixdependent mechanical regulation of adhesion formation can be evaluated using such platforms. Apart from focal adhesion complex formation, which links the ECM to the actin cytoskeleton, other integrin-mediated assemblies known as podosomes can be studied. ${ }^{275}$ These multimolecular structures are often seen in monocytic-derived cells, e.g. macrophages, as their primary adhesion complex to the substratum. The core of podosomes comprise of WASP, cortactin Arp2/Arp3 and actin filaments. ${ }^{276}$ The podosome core is surrounded by a ring of integrins and focal adhesion associated proteins, ${ }^{277}$ such as talin, paxillin and vinculin. Sheetz and coworkers found that fibroblasts, RPTP ${ }^{\alpha+++v v}$ and REF52 ${ }^{\text {ww }}$ cells, cultured on RGD modified mobile SLBs formed podosome-like assemblies without artificially elevating Src activity. ${ }^{25}$ In the case of REF52 cells, as early as 45 minutes after cell seeding, podosome-like structures were observed in $70 \%$ of the cell population, an effect not seen on RGD-modified glass surfaces. Characteristic actin clusters were seen that were positive for both vinculin and paxilin on the periphery, in fibroblast cells transfected to express the suitable markers as fluorescent fusion proteins. In addition, Arp2/Arp3 were enriched within the podosome core and podosome formation could be inhibited by addition of inhibitor CK-666. Focal adhesion formation was restored upon introduction of $\mathrm{Cr}$-line barriers spaced $1 \mu \mathrm{m}$ apart, indicating that force generation could occur against the diffusion barriers without observable podosome formation. Increasing the line spacing larger than $2 \mu \mathrm{m}$ allowed podosome formation to occur, as was the case on a dot array. In the case when cells were grown partially on $1 \mu \mathrm{m}$ spaced $\mathrm{Cr}$-line barriers and on control glass, both adhesion complexes were observed. ${ }^{25}$

\footnotetext{
${ }^{v}$ RPTP $\alpha+$ ++: mouse embryonic fibroblast cells

${ }^{w w}$ REF52: rat fibroblast cells 


\subsection{Towards in vivo application of SLBs}

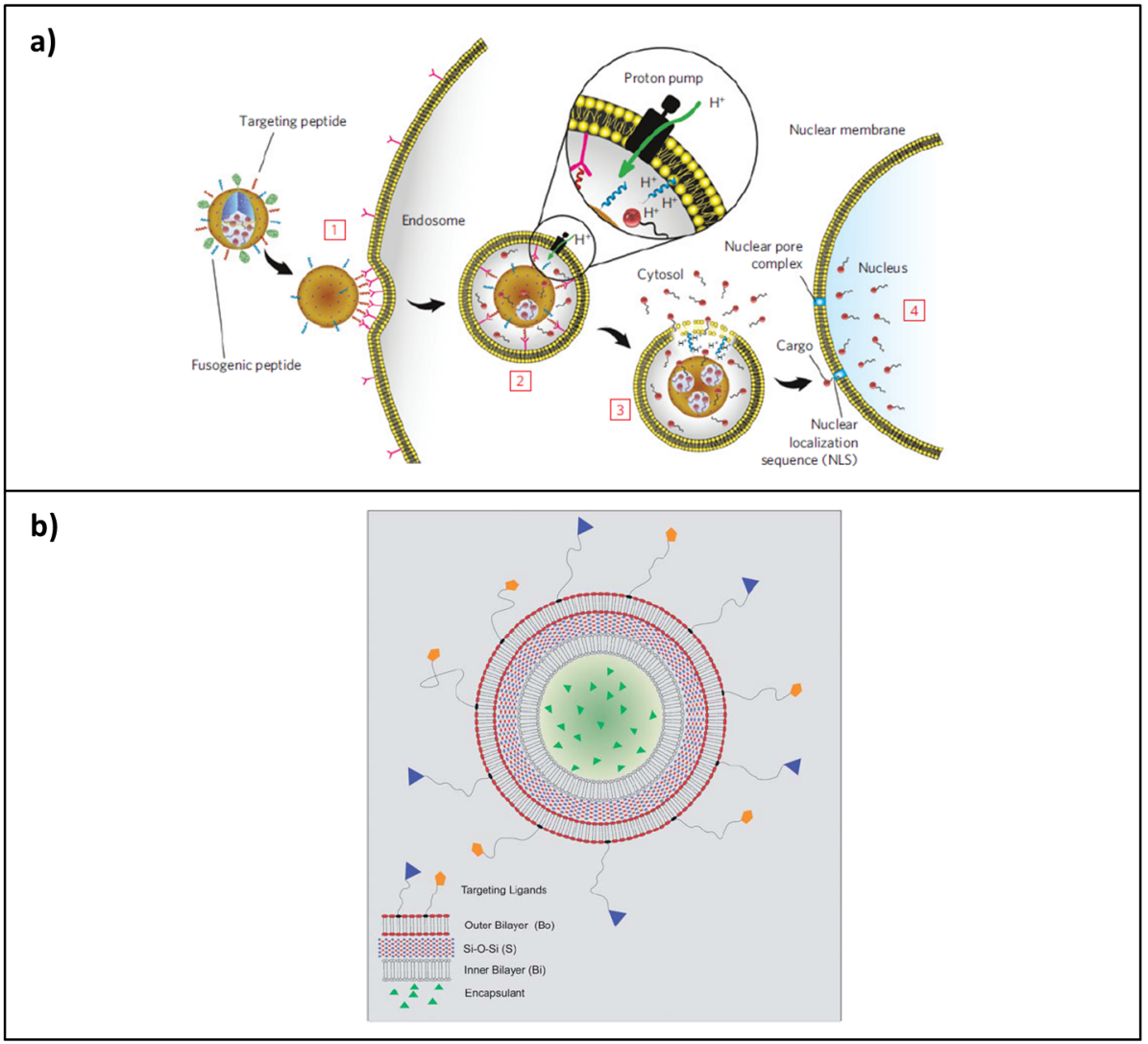

Figure 1.19: In vivo applications of SLBs; a) the protocell concept based on a mesoporous support coated with an SLB decorated with targeting ligands, PEG and fusogenic peptides for improved drug targeting and intracellular delivery. Reprinted with permission. ${ }^{278}$ Copyright 2011, Nature Publishing Group. b) Similarly, the Liposil drug delivery system is based on a drug-loaded vesicle coated with a nanoporous silica shell that served as a substrate for a second lipid bilayer decorated with targeting ligands. Reprinted with permission. ${ }^{279}$ Copyright 2011, Royal Society of Chemistry.

\subsubsection{Nanoparticle supported lipid bilayers}

To overcome problems related to poor drug solubility, stability, clearance and selectivity, Ashley and co-workers designed a multicomponent particle-SLB system, which they called protocells. ${ }^{278,279}$ The protocells consist of a mesoporous nanoparticle core coated with a SLB (Figure 1.19a). The high surface area of these mesoporous nanoparticles, $(>1,000$ $\mathrm{m}^{2} / \mathrm{g}$ ) provide a high drug loading capacity. In addition, the SLB coating prohibits nonspecific interaction and upon introduction of specific ligands, allow for targeting to cells. 
Protocells decorated with SP94 targeting peptides were evaluated for binding with $\mathrm{HCC}^{\mathrm{xx}}$ and $\mathrm{Hep}_{3} \mathrm{~B}^{\mathrm{yy}}$ cells. The $K_{\mathrm{d}}$-values for Hep3B binding for liquid-state DOPC protocells ranged from 0.94-0.08 nM at peptide densities of 6-2048 per particle. The $K_{\mathrm{d}}$-values for gel-state DPPC-based protocells were strongly dependent on the peptide density and were in general 10 times higher as compared to the mobile DOPC-based system. Additional modification of protocells with fusogenic peptide H5WYG facilitated the endosomal escape and cytosolic dispersion of encapsulated cargo. In addition, delivery to the nucleus was promoted by including a nuclear localization sequence on the cargo. ${ }^{278}$ Similarly, mesoporous silica nanoparticles coated with an SLB were used to tackle multidrug resistance (MDR) in breast cancer cells. ${ }^{280}$ The SLB was functionalized with Pluronic P123 to inhibit drug efflux in MDR breast cancer cells. Intracellular levels of the irinotecan ${ }^{z z}$ delivered via the particle-supported lipid bilayer increased 13-fold compared to free drug delivery resulting in a 7-fold increase in cytotoxicity and stronger cell cycle arrest. ${ }^{280}$ Lipidcoated particles have also been studied to improve oral drug delivery. For example, polyD, L-lactide-co-glycolide (PLGA) lipospheres were prepared by a double-emulsion method to serve as a carrier for oral protein drug delivery. ${ }^{281}$ Higher loading capacity, increased entrapment efficiency and lower burst release were observed for the lipospheres compared to the 'bare' PLGA particles. In time, transcytotic efficiency was determined with a model system for the $\mathrm{M}$-cell ${ }^{\text {aaa }}$. The results of this study showed an 1.5-fold increase compared to PLGA particles, which suggest an improved intestional absorption. $^{281}$ In addition, Parikh and co-workers proposed a drug encapsulated lipid vesicle that is coated with a sol-gel derived nanoporous silica shell to stabilize the inner vesicle. The sol-gel is subsequently modified with an outer SLB that bears targeting ligands (Figure 1.19b). To date, only the fabrication of such a system has been shown. ${ }^{279}$

\subsubsection{Lipid mimetic coating}

The use of lipid bilayers to overcome blood compatibility issues has been explored. In one example, a lipid bilayer coating was laterally stabilized using albumin, heparin and PEG on a poly methyl methacrylate (PMMA) support using carbodiimide chemistry. The results showed improved blood compatibility as indicated by reduced calcification, protein adsorption and blood-cell adhesion. The SLB was found stable under low shear stress conditions (Figure 1.20a). ${ }^{282}$ The zwitterionic phosphocholine head-group, mostly responsible for the non-fouling character of SLBs has also been mimicked in artificial polymers (Figure 1.20b). 2-Methacryloyloxyethyl phosphorylcholine (MPC) polymers have been used to prevent biofouling problems in biosensors, biomaterials and

\footnotetext{
${ }^{x x}$ HCC: human hepatocellular carcinoma epithelial cells

${ }^{\text {yy }}$ Hep3B: human hepatocellular carcinoma immune cells

${ }^{\mathrm{zz}}$ Irinotecan: chemotherapy agent

aaa $\mathrm{M}$-cell: phagocytic enterocyte microfold cell 


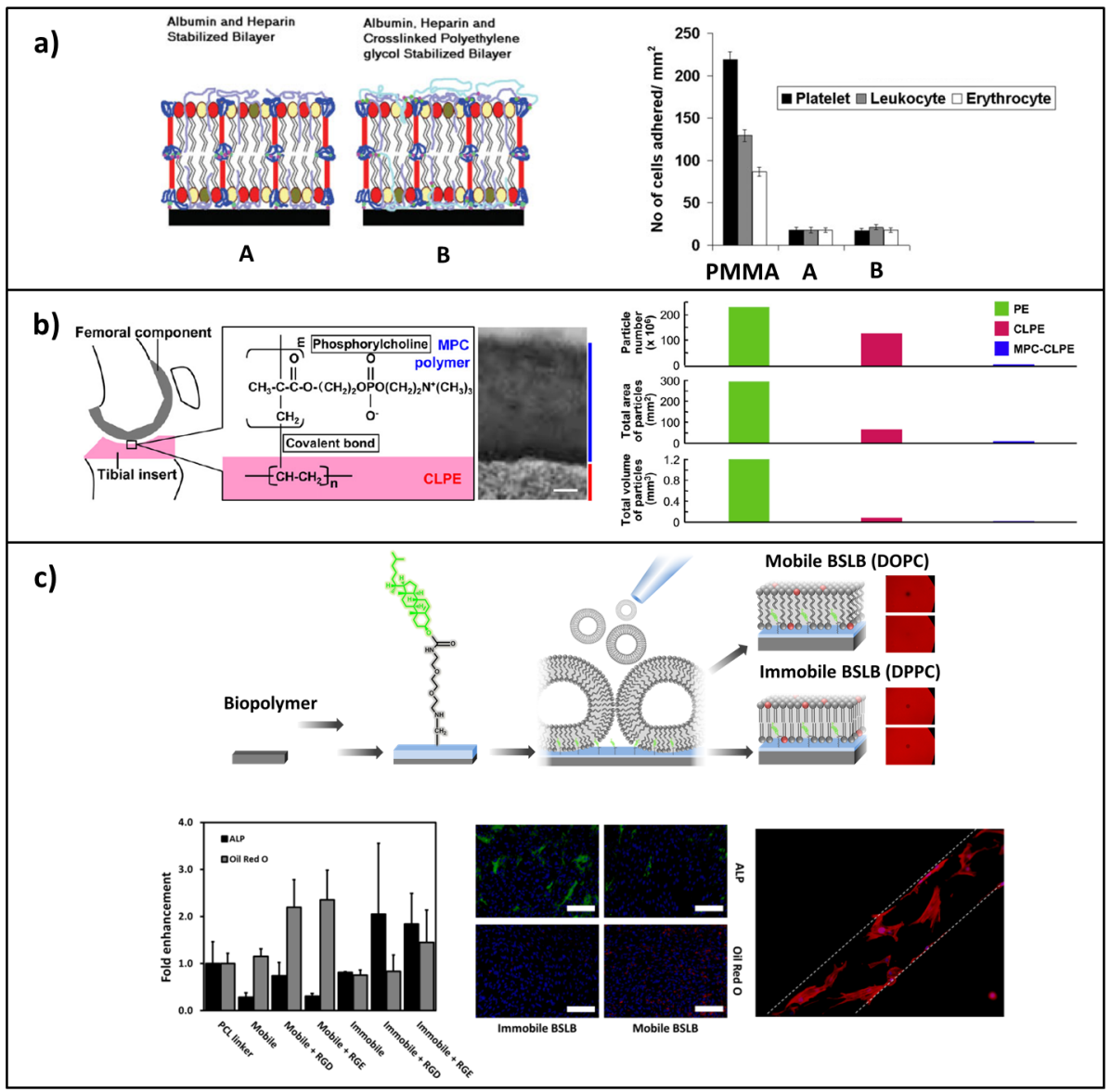

Figure 1.20: Lipid mimetic coatings; a) cross-linked SLBs using albumin and heparin or albumin, heparin and PEG on PMMA supports. Improved blood compatibility was found for both aforementioned coatings as reflected in blood cell adhesion. Reprinted with permission. ${ }^{282}$ Copyright 2006, John Wiley \& Sons. b) The use of PC-based MPC polymer as a coating for the tibial insert in TKA. Wear resistance and as a result particle formation was reduced after application of a MPC layer. Reprinted with permission. ${ }^{283}$ Copyright 2010, Elsevier. c) Concept of biomaterial supported lipid bilayer, shown to be air stable and able to provide mechanical and biological decoupling from the bulk substrate.

microfluidic systems. For example, MPC-based polymers have been evaluated for total knee arthroplasty $^{\text {bbb }}$ (TKA). In this study, the MPC polymer was grafted as the top layer onto the cross-linked polyethylene component of the knee implant. The results indicated

\footnotetext{
${ }^{\text {bbb }}$ TKA: surgical procedure to replace the knee joint with an implant substitute
} 
less wear of the articulating surfaces potentially reducing periprosthetic osteolysis and aseptic loosening ${ }^{c c c}$ of the implant. ${ }^{283}$ A detailed overview of the various applications of MPC and the fabrication of MPC-coatings has recently been described by Iwasaki and Ishihara. $^{284}$

\subsubsection{Biomaterial supported SLBs}

Despite the interesting properties posed by SLBs, their use in in vivo applications is limited and challenged by the limited stability of SLBs in air. To this end, we developed a modification strategy that is applicable to biopolymers for the fabrication of a cholesterolmodified biopolymer surface. As was shown previously, homogeneously distributed cholesterol is able to stabilize the SLB and provide air-stability. ${ }^{155}$ As described in Chapter 6 , a cholesterol molecule was synthesized that bears an triethyleneoxide linker with a primary amine. Careful control of an oxygen plasma treatment step of the biopolymer PCL facilitated the formation of aldehyde functionalities to which the cholesterol linker can be coupled via an imine bond. These imine bonds can be converted into stable secondary amines following a short and mild reduction step. After vesicle fusion on these cholesterol-modified biomaterials, the formed Biomaterial SLB (BSLB) was proven to be non-fouling, air-stable and able to provide mechanical and biological decoupling of cell responses from the substrate (Figure 1.20c). Upon varying the lateral mobility of a cell adhesive amphiphile, surface-mitigated differentiation was observed for mesenchymal stem cells. This effect could be attributed to differences in traction forces.

\subsection{Scope and outline of the thesis}

The potential of SLBs for studying receptor-ligand binding, cell adhesion and their use as a model system for cell membranes is well recognized in the area of biophysical research. ${ }^{285}$ Currently, applications of SLBs come to the fore ranging from capillary SLB coatings, biosensing on SLBs to increase understanding of cell membrane properties and organization, while SLBs have recently also shown their use as a system for influencing and studying cell behaviour in vitro. In addition, first reports appear on in vivo applications such as drug delivery systems and biomaterial coatings. The wealth of know-how present in the scientific literature and the commercial availability of lipids, amphiphiles and membrane components makes SLBs more and more accessible to research laboratories. However, to date there exists an enormous unexplored potential to tailor SLBs for membrane separation and their use as surface gradients for e.g. binding studies, their in vivo application and the combination with more complex supramolecular synthetic assemblies.

\footnotetext{
${ }^{c c c}$ Periprosthetic osteolysis and aseptic loosening: Processes involved in implants loosening 60
} 
In Chapter 1 surface attached lipid bilayers are reviewed as an attractive tool to mimic cell-cell, cell-ECM and promote cell-material interaction. Many types of surface-attached lipid bilayers are discussed such as tethered and cushioned SLB architectures as well as their characterization and applications. In Chapter 2 a method is explored to overcome problems related to the high electrical fields required in bulk membrane electrophoresis. The method enables the use of only 100s of millivolt using a chip-based system. This method of membrane electrophoresis is evaluated in Chapter 3 for the fabrication of welldefined surface gradients that are stable in time. The chemical modification of these locked-in gradients will be discussed as well in this Chapter. In Chapter 4, the use of stable SLB surface gradients, as described in Chapter 3, are used to provide insights into the binding behaviour of $E$. coli bacteria under physiologically relevant shear stress. In Chapter 5 the effect of the lateral mobility of bioactive ligands in SLBs on human primary chondrocytes and mouse progenitor cells on cell behaviour in terms of cell adhesion, spreading and cytoskeletal organization will be studied. The insights in variations in lateral mobility of ligands in relation to cell adhesive behaviour as observed in Chapter 5 is used in Chapter 6 to explore a novel coating technology for biomaterials. The introduction of SLBs on a range of biomaterials will be studied and the Chapter concludes with discussing the results of stem cell differentiation on air-stable SLB coated biomaterials. 


\subsection{References}

Jacobson, K.; Sheets, E. D.; Simson, R. Science 1995, 268, 1441-1442.

Monks, C. R.; Freiberg, B. A.; Kupfer, H.; Sciaky, N.; Kupfer, A. Nature 1998, 395, 82-86.

Maxfield, F. R. Curr. Opin. Cell Biol. 2002, 14, 483-487.

Brinkmann, J.; Cavatorta, E.; Sankaran, S.; Schmidt, B.; van Weerd, J.; Jonkheijm, P. Chem. Soc. Rev. 2014, 43, 4449-4469.

Singer, S. J.; Nicolson, G. L. Science 1972, 175, 720-731.

Engelman, D. M. Nature 2005, 438, 578-580.

Coskun, U.; Simons, K. Structure 2011, 19, 1543-1548.

Devaux, P. F. Biochemistry 1991, 30, 1163-1173.

Devaux, P. F. Annu. Rev. Biophys. Biomol. Struct. 1992, 21, 417-439.

Daleke, D. L. J. Lipid. Res. 2003, 44, 233-242.

Fadeel, B.; Xue, D. Crit. Rev. Biochem. Mol. Biol. 2009, 44, 264-277.

Brown, D. A.; Rose, J. K. Cell 1992, 68, 533-544.

Schroeder, R.; London, E.; Brown, D. Proc. Natl. Acad. Sci. USA 1994, 91, 12130-12134.

Brown, D. A. Physiology 2006, 21, 430-439.

Lingwood, D.; Simons, K. Science 2010, 327, 46-50.

Tomas, S.; Milanesi, L. Nat. Chem. 2010, 2, 1077-1083.

Chidlow, J. H., Jr.; Sessa, W. C. Cardiovasc. Res. 2010, 86, 219-225.

Jacobson, K.; Mouritsen, O. G.; Anderson, R. G. W. Nat. Cell Biol. 2007, 9, 7-14.

Levitan, I.; Gooch, K. J. Antioxid. Redox Signal. 2007, 9, 1519-1534.

Van Meer, G. EMBO Journal 2005, 24, 3159-3165.

Marsh, D. Biophys. J. 2007, 93, 3884-3899.

van Meer, G.; Voelker, D. R.; Feigenson, G. W. Nat. Rev. Mol. Cell Biol. 2008, 9, 112-124.

Ali, M. R.; Cheng, K. H.; Huang, J. Biochemistry 2006, 45, 12629-12638.

Yu, C. H.; Luo, W. W.; Sheetz, M. P. Cell Adhes. Migr. 2012, 6, 280-284.

Yu, C.-h.; Rafiq, Nisha Bte M.; Krishnasamy, A.; Hartman, Kevin L.; Jones, Gareth E.; Bershadsky, Alexander D.; Sheetz, Michael P. Cell Reports 2013, 5, 1456-1468.

Kaufman, Y.; Grinberg, S.; Linder, C.; Heldman, E.; Gilron, J.; Freger, V. Langmuir 2013, 29, 1152-1161.

Kaufman, Y.; Grinberg, S.; Linder, C.; Heldman, E.; Gilron, J.; Shen, Y.-x.; Kumar, M.; Lammertink, R. G. H.; Freger, V. J. Membr. Sci. 2014, 457, 50-61.

Andersson, M.; Jackman, J.; Wilson, D.; Jarvoll, P.; Alfredsson, V.; Okeyo, G.; Duran, R. Colloids. Surf. B. 2011, 82, 550-561.

Kaufmann, S.; Ilg, K.; Mashaghi, A.; Textor, M.; Priem, B.; Aebi, M.; Reimhult, E. Langmuir 2012, 28, 12199-12208.

Mueller, P.; Rudin, D. O.; Tien, H. T.; Wescott, W. C. Nature 1962, 194, 979-980.

Brian, A. A.; McConnell, H. M. Proc. Natl. Acad. Sci. USA 1984, 81, 6159-6163.

Tamm, L. K.; Mcconnell, H. M. Biophys. J. 1985, 47, 105-113.

Castellana, E. T.; Cremer, P. S. Surf. Sci. Rep. 2006, 61, 429-444.

Chan, Y.-H. M.; Boxer, S. G. Curr. Opin. Chem. Biol. 2007, 11, 581-587.

Yu, C. H.; Groves, J. T. Med. Biol. Eng. Comput. 2010, 48, 955-963.

Khan, M. S.; Dosoky, N. S.; Williams, J. D. Int. J. Mol. Sci. 2013, 14, 21561-21597.

Tamm, L. K. Klin. Wochenschr. 1984, 62, 502-503.

Ruozi, B.; Belletti, D.; Tombesi, A.; Tosi, G.; Bondioli, L.; Forni, F.; Vandelli, M. A. Int. J. Nanomed. 2011, 6, 557-563.

Cho, N.-J.; Hwang, L.; Solandt, J.; Frank, C. Materials 2013, 6, 3294-3308.

Koenig, B. W.; Kruger, S.; Orts, W. J.; Majkrzak, C. F.; Berk, N. F.; Silverton, J. V.; Gawrisch, K. Langmuir 1996, 12, 1343-1350. 14032. 
Sugihara, K.; Jang, B.; Schneider, M.; Voros, J.; Zambelli, T. Soft Matter 2012, 8, 5525-5531. Hardy, G. J.; Nayak, R.; Zauscher, S. Curr. Opin. Colloid Interface Sci. 2013, 18, 448-458. Kalb, E.; Frey, S.; Tamm, L. K. Biochim. Biophys. Acta 1992, 1103, 307-316.

Mennicke, U.; Salditt, T. Langmuir 2002, 18, 8172-8177.

Simonsen, A. C.; Bagatolli, L. A. Langmuir 2004, 20, 9720-9728.

Reich, C.; Andruzzi, L. Soft Matter 2010, 6, 493-500.

Mashaghi, S.; van Oijen, A. M. Biotechnol. Bioeng. 2014, 111, 2076-2081.

Knoll, W.; Koper, I.; Naumann, R.; Sinner, E. K. Electrochim. Acta 2008, 53, 6680-6689.

Jackman, J. A.; Knoll, W.; Cho, N. J. Materials 2012, 5, 2637-2657.

Oliver, A. E.; Kendall, E. L.; Howland, M. C.; Sanii, B.; Shreve, A. P.; Parikh, A. N. Lab Chip 2008, 8, 892897.

Nellis, B. A.; Satcher, J. H., Jr.; Risbud, S. H. Colloids. Surf. B. 2011, 82, 647-650.

Nellis, B. A.; Satcher, J. H., Jr.; Risbud, S. H. Acta Biomater. 2011, 7, 380-386.

Yi, F.; Xu, J.; Smith, A. M.; Parikh, A. N.; Lavan, D. A. Soft Matter 2009, 5, 5037-5041.

Tanaka, M.; Sackmann, E. Nature 2005, 437, 656-663.

Naumann, R. L. C.; Knoll, W. Biointerphases 2008, 3, Fa101-Fa107.

Knoll, W.; Naumann, R.; Friedrich, M.; Robertson, J. W. F.; Losche, M.; Heinrich, F.; McGillivray, D. J.; Schuster, B.; Gufler, P. C.; Pum, D.; Sleytr, U. B. Biointerphases 2008, 3, Fa125-Fa135.

Knoll, W. Handbook of biofunctional surfaces, 2013.

Diaz, A. J.; Albertorio, F.; Daniel, S.; Cremer, P. S. Langmuir 2008, 24, 6820-6826.

Santonicola, M. G.; Memesa, M.; Meszynska, A.; Ma, Y.; Vancso, G. J. Soft Matter 2012, 8, 1556-1562.

Shi, J.; Chen, J.; Cremer, P. S. J. Am. Chem. Soc. 2008, 130, 2718-2719.

Cremer, P. S.; Groves, J. T.; Kung, L. A.; Boxer, S. G. Langmuir 1999, 15, 3893-3896.

Groves, J. T.; Ulman, N.; Boxer, S. G. Science 1997, 275, 651-653.

Groves, J. T.; Ulman, N.; Cremer, P. S.; Boxer, S. G. Langmuir 1998, 14, 3347-3350.

Kung, L. A.; Kam, L.; Hovis, J. S.; Boxer, S. G. Langmuir 2000, 16, 6773-6776.

Kam, L.; Boxer, S. G. J. Biomed. Mater. Res. 2001, 55, 487-495.

Chalmeau, J.; le Grimellec, C.; Sternick, J.; Vieu, C. Colloids. Surf. B. 2012, 89, 188-195.

Yang, T. L.; Simanek, E. E.; Cremer, P. Anal. Chem. 2000, 72, 2587-2589.

Majd, S.; Mayer, M. Angew. Chem. Int. Ed. 2005, 44, 6697-6700.

Cremer, P. S.; Yang, T. L. J. Am. Chem. Soc. 1999, 121, 8130-8131.

Strulson, M. K.; Maurer, J. A. Langmuir 2011, 27, 12052-12057.

Howland, M. C.; Sapuri-Butti, A. R.; Dixit, S. S.; Dattelbaum, A. M.; Shreve, A. P.; Parikh, A. N. J. Am. Chem. Soc. 2005, 127, 6752-6765.

Lenz, P.; Ajo-Franklin, C. M.; Boxer, S. G. Langmuir 2004, 20, 11092-11099.

Yee, C. K.; Amweg, M. L.; Parikh, A. N. J. Am. Chem. Soc. 2004, 126, 13962-13972.

Morigaki, K.; Mizutani, K.; Saito, M.; Okazaki, T.; Nakajima, Y.; Tatsu, Y.; Imaishi, H. Langmuir 2013, 29, 2722-2730.

Janshoff, A.; Kunneke, S. Eur. Biophys. J. 2000, 29, 549-554.

Yang, T.; Jung, S.; Mao, H.; Cremer, P. S. Anal. Chem. 2001, 73, 165-169.

Yang, T.; Baryshnikova, O. K.; Mao, H.; Holden, M. A.; Cremer, P. S. J. Am. Chem. Soc. 2003, 125, 47794784.

Jung, H. S.; Yang, T.; Lasagna, M. D.; Shi, J. J.; Reinhart, G. D.; Cremer, P. S. Biophys. J. 2008, 94, 30943103.

Monson, C. F.; Cong, X.; Robison, A. D.; Pace, H. P.; Liu, C. M.; Poyton, M. F.; Cremer, P. S. J. Am. Chem. Soc. 2012, 134, 7773-7779.

Jung, H.; Robison, A. D.; Cremer, P. S. J. Struct. Biol. 2009, 168, 90-94.

Shi, J.; Yang, T.; Cremer, P. S. Anal. Chem. 2008, 80, 6078-6084.

Kam, L.; Boxer, S. G. J. Am. Chem. Soc. 2000, 122, 12901-12902.

Lenhert, S.; Sun, P.; Wang, Y.; Fuchs, H.; Mirkin, C. A. Small 2007, 3, 71-75.

Sekula, S.; Fuchs, J.; Weg-Remers, S.; Nagel, P.; Schuppler, S.; Fragala, J.; Theilacker, N.; Franzreb, M.; Wingren, C.; Ellmark, P.; Borrebaeck, C. A.; Mirkin, C. A.; Fuchs, H.; Lenhert, S. Small 2008, 4, 1785-1793. Kaufmann, S.; Sobek, J.; Textor, M.; Reimhult, E. Lab Chip 2011, 11, 2403-2410.

Neumann, J.; Hennig, M.; Wixforth, A.; Manus, S.; Radler, J. O.; Schneider, M. F. Nano Lett. 2010, 10, 2903-2908. 
Roiter, Y.; Ornatska, M.; Rammohan, A. R.; Balakrishnan, J.; Heine, D. R.; Minko, S. Langmuir 2009, 25, 6287-6299.

Parthasarathy, R.; Yu, C. H.; Groves, J. T. Langmuir 2006, 22, 5095-5099.

Kam, L. C. J. Struct. Biol. 2009, 168, 3-10.

Iversen, L.; Tu, H. L.; Lin, W. C.; Christensen, S. M.; Abel, S. M.; Iwig, J.; Wu, H. J.; Gureasko, J.; Rhodes, C.; Petit, R. S.; Hansen, S. D.; Thill, P.; Yu, C. H.; Stamou, D.; Chakraborty, A. K.; Kuriyan, J.; Groves, J. T. Science 2014, 345, 50-54.

Cho, N. J.; Frank, C. W.; Kasemo, B.; Hook, F. Nat. Protoc. 2010, 5, 1096-1106.

Sullan, R. M.; Li, J. K.; Hao, C.; Walker, G. C.; Zou, S. Biophys. J. 2010, 99, 507-516.

Axelrod, D.; Koppel, D. E.; Schlessinger, J.; Elson, E.; Webb, W. W. Biophys. J. 1976, 16, 1055-1069.

Soumpasis, D. M. Biophys. J. 1983, 41, 95-97.

Kapitza, H. G.; Mcgregor, G.; Jacobson, K. A. Proc. Natl. Acad. Sci. USA 1985, 82, 4122-4126.

Jönsson, P.; Jonsson, M. P.; Tegenfeldt, J. O.; Höök, F. Biophys. J. 2008, 95, 5334-5348.

Crane, J. M.; Tamm, L. K. Methods Mol. Biol. 2007, 400, 481-488.

Axelrod, D. J. Cell Biol. 1981, 89, 141-145.

Machan, R.; Hof, M. BBA. Biomembranes 2010, 1798, 1377-1391.

Morigaki, K.; Tawa, K. Biophys. J. 2006, 91, 1380-1387.

Stroumpoulis, D.; Parra, A.; Tirrell, M. AlChE J. 2006, 52, 2931-2937.

Cho, N. J.; Wang, G. L.; Edvardsson, M.; Glenn, J. S.; Hook, F.; Frank, C. W. Anal. Chem. 2009, 81, 47524761.

Richter, R.; Mukhopadhyay, A.; Brisson, A. Biophys. J. 2003, 85, 3035-3047.

Richter, R. P.; Brisson, A. R. Biophys. J. 2005, 88, 3422-3433.

Zhu, X.; Wang, Z.; Zhao, A.; Huang, N.; Chen, H.; Zhou, S.; Xie, X. Colloids. Surf. B. 2014, 116, 459-464.

Nussio, M. R.; Oncins, G.; Ridelis, I.; Szili, E.; Shapter, J. G.; Sanz, F.; Voelcker, N. H. J. Phys. Chem. B. 2009, 113, 10339-10347.

Canale, C.; Jacono, M.; Diaspro, A.; Dante, S. Microsc. Res. Techniq. 2010, 73, 965-972.

El Kirat, K.; Morandat, S.; Dufrene, Y. F. BBA. Biomembranes 2010, 1798, 750-765.

Mingeot-Leclercq, M. P.; Deleu, M.; Brasseur, R.; Dufrene, Y. F. Nat. Protoc. 2008, 3, 1654-1659.

Alessandrini, A.; Facci, P. Soft Matter 2014, 10, 7145-7164.

Shaw, J. E.; Slade, A.; Yip, C. M. J. Am. Chem. Soc. 2003, 125, 11838-11839.

Andersson, A. S.; Glasmastar, K.; Sutherland, D.; Lidberg, U.; Kasemo, B. J. Biomed. Mater. Res. A. 2003, 64A, 622-629.

Lozano, M. M.; Liu, Z.; Sunnick, E.; Janshoff, A.; Kumar, K.; Boxer, S. G. J. Am. Chem. Soc. 2013, 135, 5620-5630.

Pace, H. P.; Sherrod, S. D.; Monson, C. F.; Russell, D. H.; Cremer, P. S. Anal. Chem. 2013, 85, 6047-6052.

Yuan, C. B.; Furlong, J.; Burgos, P.; Johnston, L. J. Biophys. J. 2002, 82, 2526-2535.

Babayco, C. B.; Turgut, S.; Smith, A. M.; Sanii, B.; Land, D.; Parikh, A. N. Soft Matter 2010, 6, 5877-5881.

Scomparin, C.; Lecuyer, S.; Ferreira, M.; Charitat, T.; Tinland, B. Eur. Phys. J. E Soft Matter 2009, 28, 211220.

Satriano, C.; Svedhem, S.; Kasemo, B. Phys. Chem. Chem. Phys. 2012, 14, 16695-16698.

Nakashima, H.; Furukawa, K.; Kashimura, Y.; Sumitomo, K.; Shinozaki, Y.; Torimitsu, K. Langmuir 2010, 26, 12716-12721.

Wang, T. Y.; Leventis, R.; Silvius, J. R. Biochemistry 2001, 40, 13031-13040.

Levental, I.; Grzybek, M.; Simons, K. Biochemistry 2010, 49, 6305-6316.

Glasmastar, K.; Larsson, C.; Hook, F.; Kasemo, B. J. Colloid Interf. Sci. 2002, 246, 40-47.

Oliver, A. E.; Ngassam, V.; Dang, P.; Sanii, B.; Wu, H.; Yee, C. K.; Yeh, Y.; Parikh, A. N. Langmuir 2009, 25, 6992-6996.

Dixit, S. S.; Szmodis, A.; Parikh, A. N. ChemPhysChem 2006, 7, 1678-1681.

Wu, J. C.; Tseng, P. Y.; Tsai, W. S.; Liao, M. Y.; Lu, S. H.; Frank, C. W.; Chen, J. S.; Wu, H. C.; Chang, Y. C. Biomaterials 2013, 34, 5191-5199.

Li, S.; Chen, N.; Zhang, Z.; Wang, Y. Biomaterials 2013, 34, 460-469.

Andreasson-Ochsner, M.; Romano, G.; Hakanson, M.; Smith, M. L.; Leckband, D. E.; Textor, M.; Reimhult, E. Lab Chip 2011, 11, 2876-2883.

Page, J.; Heitz, B. A.; Joubert, J. R.; Keogh, J. P.; Sparer, T.; Saavedra, S. S.; He, W. J. Biomed. Mater. Res. A. 2011, 97, 212-217.

Mansfield, E.; Ross, E. E.; Aspinwall, C. A. Anal. Chem. 2007, 79, 3135-3141. 
Cunliffe, J. M.; Baryla, N. E.; Lucy, C. A. Anal. Chem. 2002, 74, 776-783.

Zhdanov, V. P.; Kasemo, B. Eur. Biophys. J. Biophy. 2010, 39, 1477-1482.

Michel, F. M.; Hosein, H. A.; Hausner, D. B.; Debnath, S.; Parise, J. B.; Strongin, D. R. BBA. General subjects 2010, 1800, 871-885.

Britt, D. W.; Mobius, D.; Hlady, V. Phys. Chem. Chem. Phys. 2000, 2, 4594-4599.

Wang, Y.; Du, X. Langmuir 2006, 22, 6195-6202.

Turner, N. W.; Wright, B. E.; Hlady, V.; Britt, D. W. J. Colloid Interf. Sci. 2007, 308, 71-80.

Conboy, J. C.; Liu, S. C.; O'Brien, D. F.; Saavedra, S. S. Biomacromolecules 2003, 4, 841-849.

Ross, E. E.; Rozanski, L. J.; Spratt, T.; Liu, S. C.; O'Brien, D. F.; Saavedra, S. S. Langmuir 2003, 19, $1752-$ 1765.

Subramaniam, V.; D'Ambruoso, G. D.; Hall, H. K.; Wysocki, R. J.; Brown, M. F.; Saavedra, S. S. Langmuir 2008, 24, 11067-11075.

Joubert, J. R.; Smith, K. A.; Johnson, E.; Keogh, J. P.; Wysocki, V. H.; Gale, B. K.; Conboy, J. C.; Saavedra, S. S. Acs. Appl. Mater. Inter. 2009, 1, 1310-1315.

Heitz, B. A.; Xu, J. H.; Jones, I. W.; Keogh, J. P.; Comi, T. J.; Hall, H. K.; Aspinwall, C. A.; Saavedra, S. S. Langmuir 2011, 27, 1882-1890.

Okazaki, T.; Inaba, T.; Tatsu, Y.; Tero, R.; Urisu, T.; Morigaki, K. Langmuir 2009, 25, 345-351.

Holden, M. A.; Jung, S. Y.; Yang, T.; Castellana, E. T.; Cremer, P. S. J. Am. Chem. Soc. 2004, 126, $6512-$ 6513.

Albertorio, F.; Diaz, A. J.; Yang, T.; Chapa, V. A.; Kataoka, S.; Castellana, E. T.; Cremer, P. S. Langmuir 2005, 21, 7476-7482.

Crowe, J. H.; Hoekstra, F. A.; Crowe, L. M. Annu. Rev. Physiol. 1992, 54, 579-599.

Crowe, L. M. Comp. Biochem. Phys. A 2002, 131, 505-513.

Albertorio, F.; Chapa, V. A.; Chen, X.; Diaz, A. J.; Cremer, P. S. J. Am. Chem. Soc. 2007, 129, 10567-10574. Wilkop, T. E.; Sanborn, J.; Oliver, A. E.; Hanson, J. M.; Parikh, A. N. J. Am. Chem. Soc. 2014, 136, 60-63. Harland, C. W.; Botyanszki, Z.; Rabuka, D.; Bertozzi, C. R.; Parthasarathy, R. Langmuir 2009, 25, 51935198.

Schuster, B.; Pum, D.; Sleytr, U. B. Biointerphases 2008, 3, Fa3-Fa11.

Schuster, B.; Sleytr, U. B. J. R. Soc. Interface 2014, 11.

Deng, Y.; Wang, Y.; Holtz, B.; Li, J.; Traaseth, N.; Veglia, G.; Stottrup, B. J.; Elde, R.; Pei, D.; Guo, A.; Zhu, X. Y. J. Am. Chem. Soc. 2008, 130, 6267-6271.

Han, C. T.; Chao, L. Acs. Appl. Mater. Inter. 2014, 6, 6378-6383.

Balashev, K.; Atanasov, V.; Mitewa, M.; Petrova, S.; Bjornholm, T. BBA. Biomembranes 2011, 1808, 191198.

Shreve, A. P.; Howland, M. C.; Sapuri-Butti, A. R.; Allen, T. W.; Parikh, A. N. Langmuir 2008, 24, 1325013253.

Richter, R. P.; Maury, N.; Brisson, A. R. Langmuir 2005, 21, 299-304.

Kunze, A.; Svedhem, S.; Kasemo, B. Langmuir 2009, 25, 5146-5158.

Pont, J. J. H. H. M. d. Molecular aspects of transport proteins; Elsevier: Amsterdam ; New York, 1992.

Watanabe, R.; Soga, N.; Fujita, D.; Tabata, K. V.; Yamauchi, L.; Hyeon Kim, S.; Asanuma, D.; Kamiya, M.; Urano, Y.; Suga, H.; Noji, H. Nat. Commun. 2014, 5, 4519.

Linden, M. V.; Wiedmer, S. K.; Hakala, R. M. S.; Riekkola, M. L. J. Chromatogr. A 2004, 1051, 61-68.

Gizeli, E.; Glad, J. Anal. Chem. 2004, 76, 3995-4001.

Nye, J. A.; Groves, J. T. Langmuir 2008, 24, 4145-4149.

Gonzalez, M.; Bagatolli, L. A.; Echabe, I.; Arrondo, J. L. R.; Argarana, C. E.; Cantor, C. R.; Fidelio, G. D. J. Biol. Chem. 1997, 272, 11288-11294.

Nair, P. M.; Salaita, K.; Petit, R. S.; Groves, J. T. Nat. Protoc. 2011, 6, 523-539.

Svedhem, S.; Dahlborg, D.; Ekeroth, J.; Kelly, J.; Hook, F.; Gold, J. Langmuir 2003, 19, 6730-6736.

Schuy, S.; Treutlein, B.; Pietuch, A.; Janshoff, A. Small 2008, 4, 970-981.

Huang, C. J.; Cho, N. J.; Hsu, C. J.; Tseng, P. Y.; Frank, C. W.; Chang, Y. C. Biomacromolecules 2010, 11, 1231-1240.

van Lengerich, B.; Rawle, R. J.; Boxer, S. G. Langmuir 2010, 26, 8666-8672.

Nguyen, U. T. T.; Guo, Z.; Delon, C.; Wu, Y. W.; Deraeve, C.; Fraenzel, B.; Bon, R. S.; Blankenfeldt, W.; Goody, R. S.; Waldmann, H.; Wolters, D.; Alexandrov, K. Nature Chemical Biology 2009, 5, 227-235.

Hougland, J. L.; Fierke, C. A. Nat. Chem. Biol. 2009, 5, 197-198.

Nadolski, M. J.; Linder, M. E. FEBS J. 2007, 274, 5202-5210. 
Weinrich, D.; Lin, P. C.; Jonkheijm, P.; Nguyen, U. T.; Schroder, H.; Niemeyer, C. M.; Alexandrov, K.; Goody, R.; Waldmann, H. Angew Chem Int Ed Engl 2010, 49, 1252-1257.

Boutin, J. A. Cell Signal 1997, 9, 15-35.

Yamauchi, S.; Fusada, N.; Hayashi, H.; Utsumi, T.; Uozumi, N.; Endo, Y.; Tozawa, Y. FEBS J. 2010, 277, 3596-3607.

Pakalns, T.; Haverstick, K. L.; Fields, G. B.; McCarthy, J. B.; Mooradian, D. L.; Tirrell, M. Biomaterials 1999, 20, 2265-2279.

Englund, P. T. Annu. Rev. Biochem 1993, 62, 121-138.

Grakoui, A.; Bromley, S. K.; Sumen, C.; Davis, M. M.; Shaw, A. S.; Allen, P. M.; Dustin, M. L. Science 1999, 285, 221-227.

Achalkumar, A. S.; Bushby, R. J.; Evans, S. D. Soft Matter 2010, 6, 6036-6051.

Nymeyer, H.; Woolf, T. B.; Garcia, A. E. Proteins 2005, 59, 783-790.

Ganchev, D. N.; Rijkers, D. T. S.; Snel, M. M. E.; Killian, J. A.; de Kruijff, B. Biochemistry 2004, 43, $14987-$ 14993.

Rigaud, J. L.; Paternostre, M. T.; Bluzat, A. Biochemistry 1988, 27, 2677-2688.

Wagner, M. L.; Tamm, L. K. Biophys. J. 2000, 79, 1400-1414.

Shen, H. H.; Lithgow, T.; Martin, L. L. Int. J. Mol. Sci. 2013, 14, 1589-1607.

van der Meulen, S. A.; Dubacheva, G. V.; Dogterom, M.; Richter, R. P.; Leunissen, M. E. Langmuir 2014, 30, 6525-6533.

Pallarola, D.; Bochen, A.; Boehm, H.; Rechenmacher, F.; Sobahi, T. R.; Spatz, J. P.; Kessler, H. Adv. Funct. Mater. 2014, 24, 943-956.

Poo, M.; Robinson, K. R. Nature 1977, 265, 602-605.

Groves, J. T.; Boxer, S. G. Biophys. J. 1995, 69, 1972-1975.

Groves, J. T.; Boxer, S. G.; McConnel, H. M. Proc. Natl. Acad. Sci. USA 1997, 94, 13390-13395.

Monson, C. F.; Pace, H. P.; Liu, C.; Cremer, P. S. Anal. Chem. 2011, 83, 2090-2096.

Liu, C. M.; Monson, C. F.; Yang, T. L.; Pace, H.; Cremer, P. S. Anal. Chem. 2011, 83, 7876-7880.

van Weerd, J.; Krabbenborg, S. O.; Eijkel, J.; Karperien, M.; Huskens, J.; Jonkheijm, P. J. Am. Chem. Soc. 2014, 136, 100-103.

Bao, P.; Cheetham, M. R.; Roth, J. S.; Blakeston, A. C.; Bushby, R. J.; Evans, S. D. Anal. Chem. 2012, 84, 10702-10707.

Krabbenborg, S. O.; van Weerd, J.; Karperien, M.; Jonkheijm, P.; Huskens, J. ChemPhysChem 2014, 15, 3460-3465.

Cheetham, M. R.; Bramble, J. P.; McMillan, D. G. G.; Krzeminski, L.; Han, X.; Johnson, B. R. G.; Bushby, R. J.; Olmsted, P. D.; Jeuken, L. J. C.; Marritt, S. J.; Butt, J. N.; Evans, S. D. J. Am. Chem. Soc. 2011, 133, 65216524.

Cheetham, M. R.; Bramble, J. P.; McMillan, D. G. G.; Bushby, R. J.; Olmsted, P. D.; Jeuken, L. J. C.; Evans, S. D. Soft Matter 2012, 8, 5459-5465.

Hu, S. K.; Hsiao, S. W.; Mao, H. Y.; Chen, Y. M.; Chang, Y.; Chao, L. Sci. Technol. Adv. Mat. 2013, 14. Jonsson, P.; Gunnarsson, A.; Hook, F. Anal. Chem. 2011, 83, 604-611.

Johansson, B.; Olsson, T.; Jonsson, P.; Hook, F. Soft Matter 2013, 9, 9414-9419.

Jahn, R.; Fasshauer, D. Nature 2012, 490, 201-207.

Yoshina-Ishii, C.; Boxer, S. G. J. Am. Chem. Soc. 2003, 125, 3696-3697.

Chung, M.; Boxer, S. G. Langmuir 2011, 27, 5492-5497.

Chung, M.; Lowe, R. D.; Chan, Y. H. M.; Ganesan, P. V.; Boxer, S. G. J. Struct. Biol. 2009, 168, 190-199.

Rawle, R. J.; van Lengerich, B.; Chung, M.; Bendix, P. M.; Boxer, S. G. Biophys. J. 2011, 101, L37-L39.

Dutta, D.; Pulsipher, A.; Luo, W.; Yousaf, M. N. J. Am. Chem. Soc. 2011, 133, 8704-8713.

Sarkar, D.; Vemula, P. K.; Zhao, W.; Gupta, A.; Karnik, R.; Karp, J. M. Biomaterials 2010, 31, 5266-5274.

Lundstrom, K. Cell. Mol. Life Sci. 2006, 63, 2597-2607.

Martinez, J. A.; Misra, N.; Wang, Y.; Stroeve, P.; Grigoropoulos, C. P.; Noy, A. Nano Lett. 2009, 9, 11211126.

Misra, N.; Martinez, J. A.; Huang, S. C.; Wang, Y.; Stroeve, P.; Grigoropoulos, C. P.; Noy, A. Proc. Natl. Acad. Sci. USA 2009, 106, 13780-13784.

Bally, M.; Bailey, K.; Sugihara, K.; Grieshaber, D.; Voros, J.; Stadler, B. Small 2010, 6, 2481-2497.

Schmitt, E. K.; Weichbrodt, C.; Steinem, C. Soft Matter 2009, 5, 3347-3353.

Vogel, S. K.; Petrasek, Z.; Heinemann, F.; Schwille, P. eLife 2013, 2, e00116.

Richter, R. P.; Him, J. L.; Tessier, B.; Tessier, C.; Brisson, A. R. Biophys. J. 2005, 89, 3372-3385. 
Loose, M.; Schwille, P. J. Struct. Biol. 2009, 168, 143-151.

Vogel, S. K.; Schwille, P. Curr. Opin. Biotechnol. 2012, 23, 758-765.

Rivas, G.; Vogel, S. K.; Schwille, P. Curr. Opin. Chem. Biol. 2014, 22C, 18-26.

Barfoot, R. J.; Sheikh, K. H.; Johnson, B. R.; Colyer, J.; Miles, R. E.; Jeuken, L. J.; Bushby, R. J.; Evans, S. D. Langmuir 2008, 24, 6827-6836.

Lee, K.; Gallop, J. L.; Rambani, K.; Kirschner, M. W. Science 2010, 329, 1341-1345.

Liu, A. P.; Richmond, D. L.; Maibaum, L.; Pronk, S.; Geissler, P. L.; Fletcher, D. A. Nat. Phys. 2008, 4, 789793.

Merkle, D.; Kahya, N.; Schwille, P. ChemBioChem 2008, 9, 2673-2681.

Reviakine, I.; Bergsma-Schutter, W.; Brisson, A. J. Struct. Biol. 1998, 121, 356-362.

Bouter, A.; Gounou, C.; Berat, R.; Tan, S.; Gallois, B.; Granier, T.; d'Estaintot, B. L.; Poschl, E.; Brachvogel, B.; Brisson, A. R. Nat. Commun. 2011, 2.

Perez, T. D.; Nelson, W. J.; Boxer, S. G.; Kam, L. Langmuir 2005, 21, 11963-11968.

Britten, C. D. Mol. Cancer Ther. 2004, 3, 1335-1342.

Nam, J. M.; Nair, P. M.; Neve, R. M.; Gray, J. W.; Groves, J. T. ChemBioChem 2006, 7, 436-440.

Pierschbacher, M. D.; Ruoslahti, E. Nature 1984, 309, 30-33.

Ruoslahti, E.; Pierschbacher, M. D. Cell 1986, 44, 517-518.

Ruoslahti, E. Annu. Rev. Cell Dev. Biol. 1996, 12, 697-715.

Haubner, R.; Gratias, R.; Diefenbach, B.; Goodman, S. L.; Jonczyk, A.; Kessler, H. J. Am. Chem. Soc. 1996, 118, 7461-7472.

Hersel, U.; Dahmen, C.; Kessler, H. Biomaterials 2003, 24, 4385-4415.

Korner, A.; Deichmann, C.; Rossetti, F. F.; Kohler, A.; Konovalov, O. V.; Wedlich, D.; Tanaka, M. Plos One 2013, 8.

Charnley, M.; Kroschewski, R.; Textor, M. Integr. Biol. 2012, 4, 1059-1071.

Evans, S. F.; Docheva, D.; Bernecker, A.; Colnot, C.; Richter, R. P.; Tate, M. L. K. Biomaterials 2013, 34, 1878-1887.

Powell, S. K.; Rao, J.; Roque, E.; Nomizu, M.; Kuratomi, Y.; Yamada, Y.; Kleinman, H. K. J. Neurosci. Res. 2000, 61, 302-312.

Thid, D.; Bally, M.; Holm, K.; Chessari, S.; Tosatti, S.; Textor, M.; Gold, J. Langmuir 2007, 23, 1169311704.

Thid, D.; Holm, K.; Eriksson, P. S.; Ekeroth, J.; Kasemo, B.; Gold, J. J. Biomed. Mater. Res. A. 2008, 84A, 940-953.

Ananthanarayanan, B.; Little, L.; Schaffer, D. V.; Healy, K. E.; Tirrell, M. Biomaterials 2010, 31, 87068715.

Sandrin, L.; Coche-Guerente, L.; Bernstein, A.; Basit, H.; Labbe, P.; Dumy, P.; Boturyn, D. Org. Biomol. Chem. 2010, 8, 1531-1534.

Berat, R.; Remy-Zolghadry, M.; Gounou, C.; Manigand, C.; Tan, S.; Salto, C.; Arenas, E.; Bordenave, L.; Brisson, A. R. Biointerphases 2007, 2, 165-172.

Huang, C. J.; Tseng, P. Y.; Chang, Y. C. Biomaterials 2010, 31, 7183-7195.

Kleinman, H. K.; Wilkes, C. M.; Martin, G. R. Biochemistry 1981, 20, 2325-2330.

Albersdorfer, A.; Sackmann, E. Eur Phys J B 1999, 10, 663-672.

Sengupta, K.; Schilling, J.; Marx, S.; Markus, F.; Sackmann, E. Biophys. J. 2003, 84, 381a-381a.

Richter, R. P.; Hock, K. K.; Burkhartsmeyer, J.; Boehm, H.; Bingen, P.; Wang, G.; Steinmetz, N. F.; Evans, D. J.; Spatz, J. P. J. Am. Chem. Soc. 2007, 129, 5306-5307.

Altgarde, N.; Becher, J.; Moller, S.; Weber, F. E.; Schnabelrauch, M.; Svedhem, S. J. Colloid Interf. Sci. 2013, 390, 258-266.

Arnold, M.; Cavalcanti-Adam, E. A.; Glass, R.; Blummel, J.; Eck, W.; Kantlehner, M.; Kessler, H.; Spatz, J. P. ChemPhysChem 2004, 5, 383-388.

Simon, A.; Gounou, C.; Tan, S.; Tiefenauer, L.; Di Berardino, M.; Brisson, A. R. BBA. Biomembranes 2013, 1828, 2739-2744.

Afanasenkau, D.; Offenhausser, A. Langmuir 2012, 28, 13387-13394.

Groves, J. T.; Mahal, L. K.; Bertozzi, C. R. Langmuir 2001, 17, 5129-5133.

Stroumpoulis, D.; Zhang, H. N.; Rubalcava, L.; Gliem, J.; Tirrell, M. Langmuir 2007, 23, 3849-3856.

Kiessling, L. L.; Gestwicki, J. E.; Strong, L. E. Curr. Opin. Chem. Biol. 2000, 4, 696-703.

Mammen, M.; Choi, S. K.; Whitesides, G. M. Angew. Chem. Int. Ed. 1998, 37, 2755-2794.

Liang, P. H.; Wu, C. Y.; Greenberg, W. A.; Wong, C. H. Curr. Opin. Chem. Biol. 2008, 12, 86-92. 
Bouckaert, J.; Mackenzie, J.; de Paz, J. L.; Chipwaza, B.; Choudhury, D.; Zavialov, A.; Mannerstedt, K.; Anderson, J.; Pierard, D.; Wyns, L.; Seeberger, P. H.; Oscarson, S.; De Greve, H.; Knight, S. D. Mol. Microbiol. 2006, 61, 1556-1568.

Liang, P. H.; Wang, S. K.; Wong, C. H. J. Am. Chem. Soc. 2007, 129, 11177-11184.

Pieters, R. J. Org. Biomol. Chem. 2009, 7, 2013-2025.

Zhu, X. Y.; Holtz, B.; Wang, Y.; Wang, L.-X.; Orndorff, P. E.; Guo, A. J. Am. Chem. Soc. 2009, 131, 1364613650.

Thomas, W. E.; Trintchina, E.; Forero, M.; Vogel, V.; Sokurenko, E. V. Cell 2002, 109, 913-923.

Tsai, J.; Kam, L. C. Cell. Mol. Bioeng. 2010, 3, 84-90.

Glass, R.; Moller, M.; Spatz, J. P. Nanotechnology 2003, 14, 1153-1160.

Lohmuller, T.; Triffo, S.; O'Donoghue, G. P.; Xu, Q.; Coyle, M. P.; Groves, J. T. Nano Lett. 2011, 11, 49124918.

Roiter, Y.; Ornatska, M.; Rammohan, A. R.; Balakrishnan, J.; Heine, D. R.; Minko, S. Nano Lett. 2008, 8, 941-944.

Salaita, K.; Nair, P. M.; Petit, R. S.; Neve, R. M.; Das, D.; Gray, J. W.; Groves, J. T. Science 2010, 327, 13801385.

Xu, Q.; Lin, W. C.; Petit, R. S.; Groves, J. T. Biophys. J. 2011, 101, 2731-2739.

Mossman, K. D.; Campi, G.; Groves, J. T.; Dustin, M. L. Science 2005, 310, 1191-1193.

Hartman, N. C.; Nye, J. A.; Groves, J. T. Proc. Natl. Acad. Sci. USA 2009, 106, 12729-12734.

Manz, B. N.; Jackson, B. L.; Petit, R. S.; Dustin, M. L.; Groves, J. Proc. Natl. Acad. Sci. USA 2011, 108, 9089-9094.

Shen, K. Y.; Tsai, J.; Shi, P.; Kam, L. C. J. Am. Chem. Soc. 2009, 131, 13204-13205.

Lackmann, M.; Boyd, A. W. Sci. Signal. 2008, 1, re2.

Davis, S.; Gale, N. W.; Aldrich, T. H.; Maisonpierre, P. C.; Lhotak, V.; Pawson, T.; Goldfarb, M.; Yancopoulos, G. D. Science 1994, 266, 816-819.

Janes, P. W.; Saha, N.; Barton, W. A.; Kolev, M. V.; Wimmer-Kleikamp, S. H.; Nievergall, E.; Blobel, C. P.; Himanen, J. P.; Lackmann, M.; Nikolov, D. B. Cell 2005, 123, 291-304.

Yu, C. H.; Law, J. B. K.; Suryana, M.; Low, H. Y.; Sheetz, M. P. Proc. Natl. Acad. Sci. USA 2011, 108, 2058520590.

Calle, Y.; Burns, S.; Thrasher, A. J.; Jones, G. E. Eur. J. Cell Biol. 2006, 85, 151-157.

Gimona, M.; Buccione, R.; Courtneidge, S. A.; Linder, S. Curr. Opin. Cell Biol. 2008, 20, 235-241.

Zamir, E.; Geiger, B. J. Cell Sci. 2001, 114, 3583-3590.

Ashley, C. E.; Carnes, E. C.; Phillips, G. K.; Padilla, D.; Durfee, P. N.; Brown, P. A.; Hanna, T. N.; Liu, J.; Phillips, B.; Carter, M. B.; Carroll, N. J.; Jiang, X.; Dunphy, D. R.; Willman, C. L.; Petsev, D. N.; Evans, D. G.; Parikh, A. N.; Chackerian, B.; Wharton, W.; Peabody, D. S.; Brinker, C. J. Nat. Mater. 2011, 10, 389-397.

Shen, S.; Kendall, E.; Oliver, A.; Ngassam, V.; Hu, D.; Parikh, A. N. Soft Matter 2011, 1001-1005.

Zhang, X.; Li, F.; Guo, S.; Chen, X.; Wang, X.; Li, J.; Gan, Y. Biomaterials 2014, 35, 3650-3665.

Ma, T.; Wang, L.; TingyuanYang; Wang, D.; Ma, G.; Wang, S. Colloids. Surf. B. 2014, 117, 512-519.

Kaladhar, K.; Sharma, C. P. J. Biomed. Mater. Res. A. 2006, 79A, 23-35.

Moro, T.; Takatori, Y.; Kyomoto, M.; Ishihara, K.; Saiga, K.; Nakamura, K.; Kawaguchi, H. Osteoarthr. Cartil. 2010, 18, 1174-1182.

Iwasaki, Y.; Ishihara, K. Sci. Technol. Adv. Mat. 2012, 13.

Sackmann, E. Science 1996, 271, 43-48. 


\section{Chapter 2}

\section{On-Chip Electrophoresis in Supported Lipid Bilayer Membranes Achieved Using Low Potentials*}

A micro supported lipid bilayer (SLB) electrophoresis method was developed, which functions at low potentials and appreciable operating times. To this end, (hydroxymethyl)ferrocene $\left(\mathrm{F}_{\mathrm{c}} \mathrm{CH}_{2} \mathrm{OH}\right)$ was employed to provide an electrochemical reaction at the anode and cathode at low applied potentials to avoid electrolysis of water. The addition of $\mathrm{F}_{\mathrm{c}} \mathrm{CH}_{2} \mathrm{OH}$ did not alter the SLB characteristics or affect biomolecule function, and $\mathrm{pH}$ and temperature variations and bubble formation were eliminated. Applying potentials of 0.25 - $1.2 \mathrm{~V}$ during flow gave homogeneous electrical fields and a fast, reversible, and strong build-up of a charged dye-modified lipid in the direction of the oppositely charged electrode. Moreover, the mobility of streptavidin on the SLB could be modulated. This method paves the way for further developing analytical SLB-based devices.

${ }^{*}$ Part of this chapter has been published in: J. van Weerd ${ }^{\ddagger}$, S.O. Krabbenborg ${ }^{\ddagger}$, J. Eijkel, M. Karperien, J. Huskens and P. Jonkheijm; J. Am. Chem. Soc. (2014) vol. 136, p. $100-103$ ('equal authorship) 


\subsection{Introduction}

Supported lipid bilayers (SLBs) are a rewarding mimetic system for cell membranes. ${ }^{1,2}$ Employing such SLBs promotes investigations of membrane associated processes in cells by e.g. membrane proteins because they can be reconstituted in SLBs in analogy to their native membranous environment. ${ }^{1-6}$ When external electric fields are applied in SLBs, membrane proteins can be manipulated along the SLB. ${ }^{7,8}$ Using DC fields, charged membrane components could be separated either by electrophoretic or electro-osmotic motion of the charged analytes, which could be confined into small regions or near barriers. $^{9-13}$ Whilst these experiments confirm the versatility of SLBs to serve as biomimetic systems, performing membrane electrophoresis with fields of commonly 10 $20 \mathrm{~V} / \mathrm{cm}$ requires sending DC currents for a prolonged time which typically causes electrolysis of water. This leads to changes in $\mathrm{pH}$ and causes bubble formation, while concomitant changes in temperature can irreversibly affect SLB integrity and protein structure. $^{14}$

To eliminate those effects in electrophoresis set-ups, low ionic strength solutions, reduction of the aqueous volume above the SLB and high flow speeds over the SLB have been employed. ${ }^{15}$ In addition, membrane traps have been reported that utilize AC electric fields and asymmetric surface patterns to confine charged species over large distances and to retard diffusive recovery while reducing the applied potential. ${ }^{16}$ In a recent example Evans and co-workers report the use of $A C$ electric fields and embedded electrodes to further minimize the applied potential from $200 \mathrm{~V}$ to $13 \mathrm{~V}$ by reducing the inter-electrode spacing. ${ }^{17}$

In this chapter an electrochemical method is described that allows for the use of unprecedented low applied potentials of a few 100's of $\mathrm{mV}$ to induce electrophoretic migration of charged membrane components. Here, appreciable electric fields of up to 16 $\mathrm{V} / \mathrm{cm}$ were achieved while obtaining equal DC build-up times as reported before (ca. 20 minutes). ${ }^{12,17}$ This was made possible through the addition of an electro-active but electroneutral species that enabled Faradaic currents to flow without water electrolysis at the electrodes. As a result, bubble formation, temperature and $\mathrm{pH}$ fluctuations were avoided. 


\subsection{Results and discussion}

Our $\mu$ SLB electrophoresis chip is depicted in Figure 2.1. A photograph of the experimental set-up and chip design are given in Figure 2.1a. The chip has interdigitated electrodes (spaced $500 \mu \mathrm{m}$ ) and $\mathrm{Cr}$-corrals (spaced $100 \mu \mathrm{m}$ ) to enable parallel analysis. The $\mathrm{Cr}$-corrals act as diffusion barriers thereby enabling build-up of charged membranes components while applying an electric field (Figure 2.1b).

a)

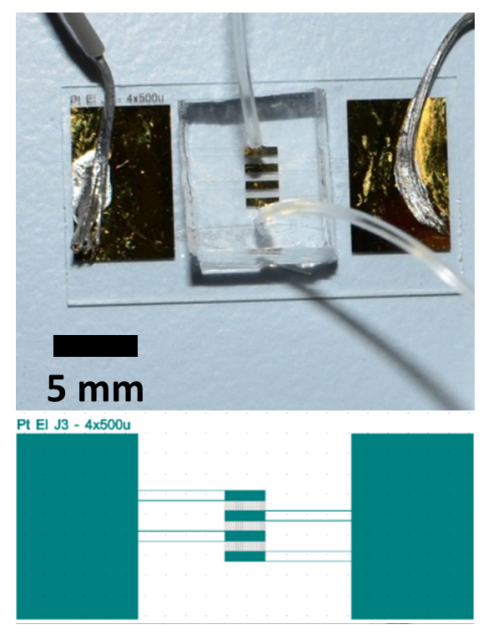

b)

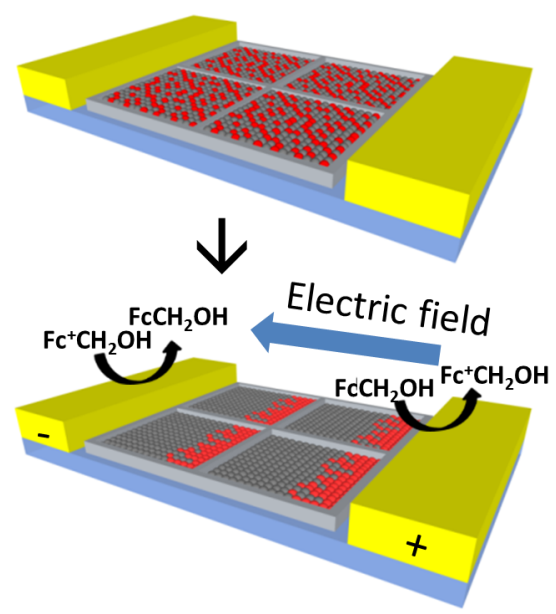

Figure 2.1: $\mu \mathrm{SLB}$ electrophoresis; a) photograph of the experimental set-up and chip design. b) Graphical representation of a single electrode junction.

The $\mu S L B$ electrophoresis chips were fabricated using a bilayer lift-off recipe in conjunction with e-beam evaporation. The Au interdigitated electrodes were prepared on borofloat glass wafers. Afterwards $\mathrm{Cr}$-corrals were fabricated in between the Au-electrodes. Subsequently, a PDMS flow channel was bonded to the chip surface to facilitate SLB formation and allow for fluid exchange. In this Chapter, a model SLB was chosen that consisted of zwitterionic DOPC and negatively charged and fluorescent TR-DHPE (Figure 2.2a). Dynamic light scattering (DLS) of the lipid vesicles is presented in Figure 2.2b. 
a)
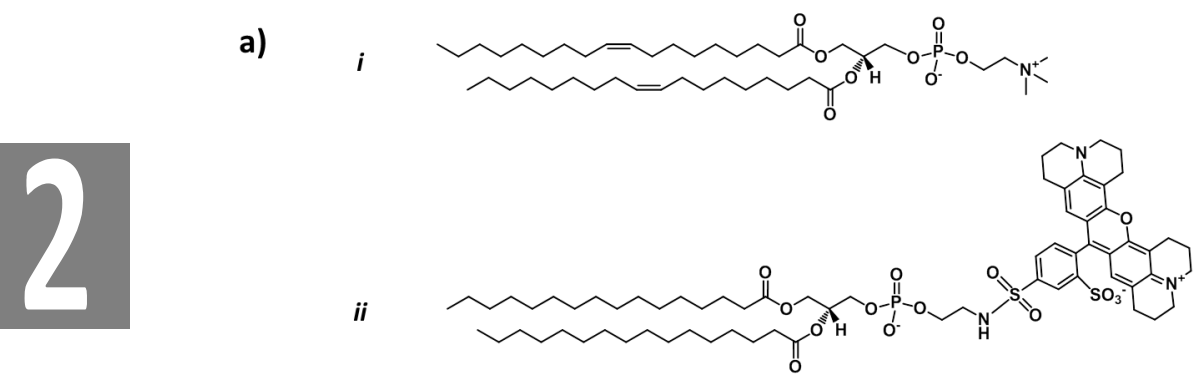

b)

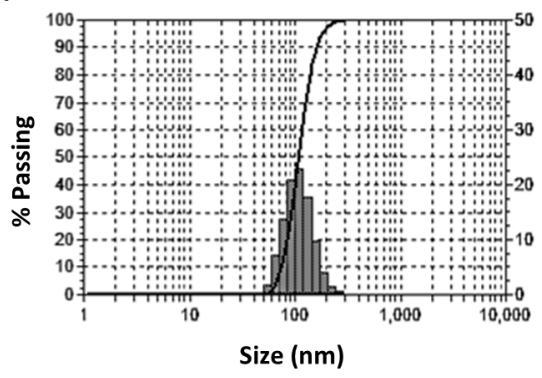

c)

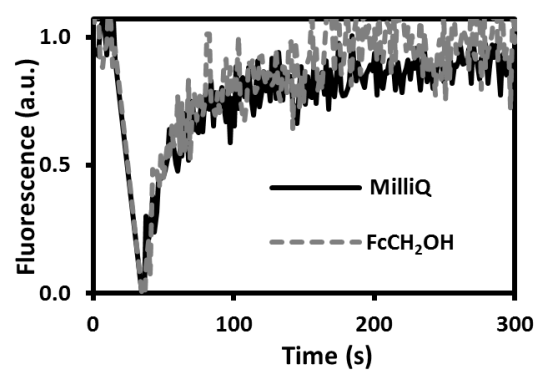

Figure 2.2: $\underline{D O P C}$ characteristics; a) chemical structures of, $i$, DOPC and, ii, TR-DHPE. b) DLS measurement of DOPC large unilaminar vesicles (LUVs) doped with $0.2 \mathrm{~mol} \%$ TR-DHPE at r.t. c) FRAP recovery curves. Comparison between MilliQ water and aqueous $0.5 \mathrm{mM} \mathrm{F}_{\mathrm{c}} \mathrm{CH}_{2} \mathrm{OH}$.

In this study, (hydroxymethyl)-ferrocene $\left(\mathrm{F}_{\mathrm{c}} \mathrm{CH}_{2} \mathrm{OH}\right)$ was selected as a water soluble electro-active but electro-neutral species, showing limited effect on the conductivity of the solution and no effect on the SLB characteristics (Figure 2.2c). The diffusion constant of the lipids in the SLBs was determined using fluorescence recovery after photobleaching (FRAP) and found to be $2.1 \pm 0.6 \mu \mathrm{m}^{2} / \mathrm{s}$ and $2.4 \pm 0.5 \mu \mathrm{m}^{2} / \mathrm{s}$ with or without $\mathrm{F}_{\mathrm{c}} \mathrm{CH}_{2} \mathrm{OH}$, respectively, while the mobile fraction approached $100 \%$ in both cases. 
Electrophoretic migration was monitored using epi-fluorescence microscopy. Representative 3D and corresponding 2D fluorescence micrographs are shown in Figure 2.3a, the latter images are recorded prior to and after applying a $1 \mathrm{~V}$ potential difference in the presence of $0.5 \mathrm{mM} \mathrm{F}_{\mathrm{c}} \mathrm{CH}_{2} \mathrm{OH}$.

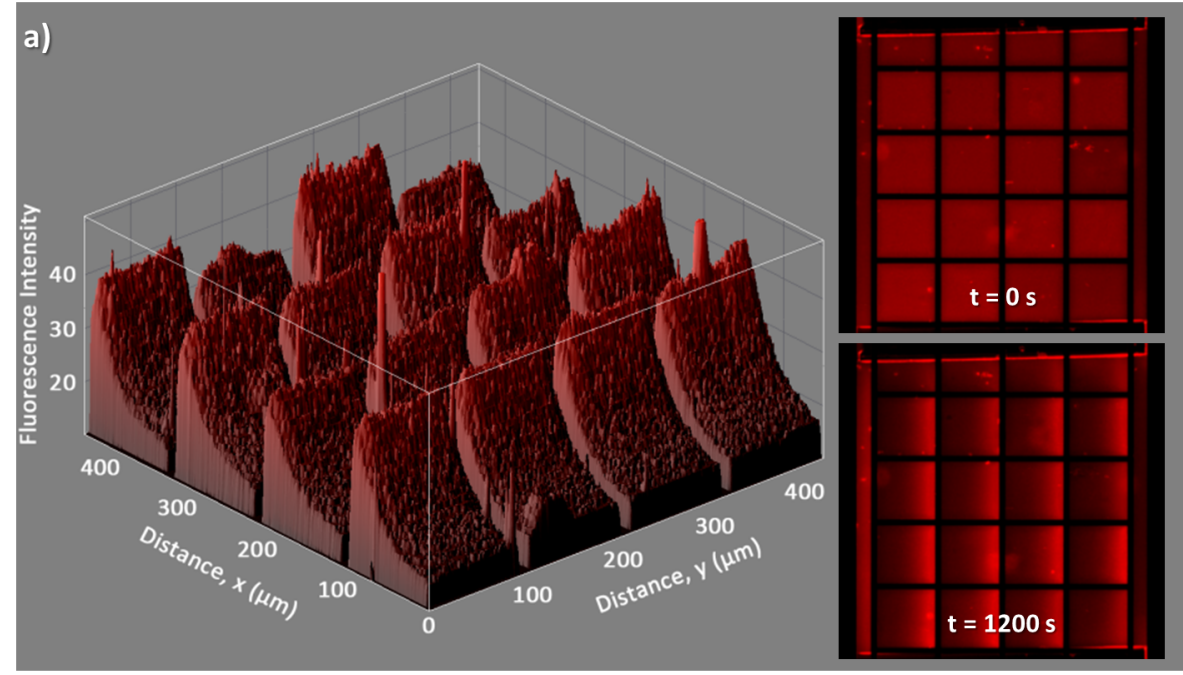

b)

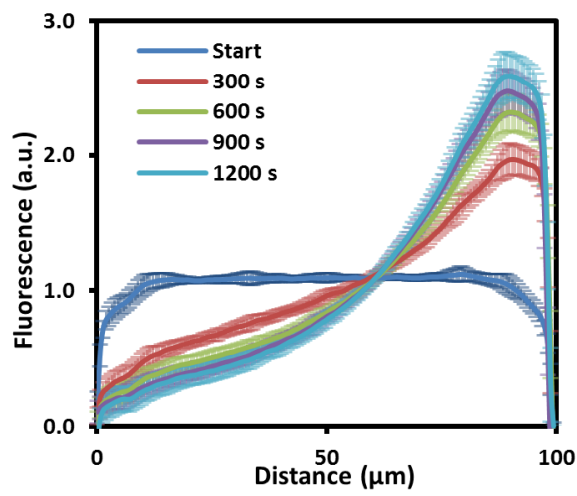

c)

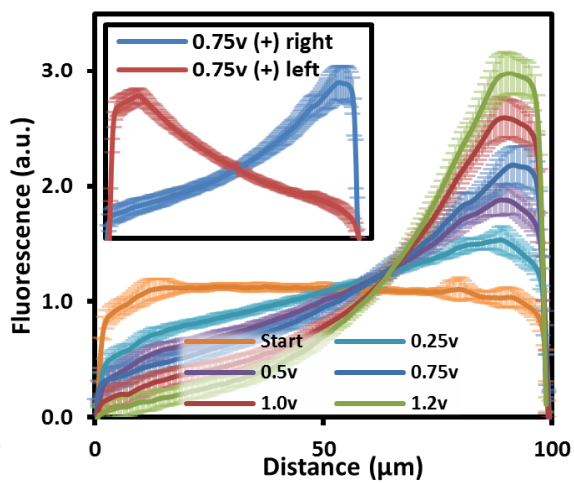

Figure 2.3: Electrophoretic build-up of TR-DHPE; a) 3D fluorescence microscopy image showing the electrophoretic build-up of a TR-DHPE containing SLB after applying $1 \mathrm{~V}$ for 20 minutes, in the presence of $0.5 \mathrm{mM} \mathrm{F}_{\mathrm{c}} \mathrm{CH}_{2} \mathrm{OH}$. Corresponding 2D fluorescence microscopy images are shown at time point 0 and 20 minutes. b) Electrophoretic build-up of TR-DHPE over time using a $1 \mathrm{~V}$ potential difference. c) Graph showing the relative TR-DHPE fluorescence intensity for different potentials. Inset shows reversal of the direction of electrophoretic migration by reversing the polarity of the electrodes. Data is presented as mean \pm SD. 
Upon applying a potential, negatively charged TR-DHPE accumulates at the Cr-barriers in the direction of the positive electrode to reach a steady-state within 20 minutes. Here, $\mathrm{t}_{1 / 2}$ is ca. 5 minutes (Figure 2.3b). When no $\mathrm{F}_{\mathrm{c}} \mathrm{CH}_{2} \mathrm{OH}$ was added, no electrophoretic migration was observed at these low applied potentials. Interestingly, upon close inspection of the fluorescence images, uniform exponential intensity profiles were observed irrespective of the corral-electrode distance. This observation suggests the presence of a homogeneous electric field in the solution. The extent of electrophoretic build-up was tuned by varying the applied potential from 0.25 to $1.2 \mathrm{~V}$ (Figure 2.3c), all well below the potential of electrolysis of water. Furthermore, the direction of electrophoretic migration was easily switched by reversing the polarity of the electrodes (inset Figure 2.3c) while the flow direction was kept constant in this experiment. The obtained concentration profiles were mirror images of each other. When a chip consisting of only two electrodes was used, electrophoretic mobility was observed only opposite to the direction of the flow. A single junction arrangement requires the first encountered electrode to be the anode since there is no oxidized $\mathrm{F}_{\mathrm{c}} \mathrm{CH}_{2} \mathrm{OH}$ present, thus fixing electrode polarity and migration direction. In contrast, an interdigitated array provides both oxidized and reduced forms of $\mathrm{F}_{\mathrm{c}} \mathrm{CH}_{2} \mathrm{OH}$ to flow over the SLB irrespective of electrode polarity, thus enabling AC applications.

To demonstrate that our technique is compatible with biomolecules and their function, streptavidin (SAv) binding to a biotinylated SLB was studied in situ, a commonly used model system. To this end, the SLB was doped with 1 mol\% of biotinyl-PE to allow for binding of fluorescently labelled SAv. As an internal control $0.2 \mathrm{~mol} \%$ of TR-DHPE was included as well. The formation of the biotinylated SLB and fluidity on the chip were confirmed with FRAP (Figure 2.4a). Subsequently, Alexa488-labelled SAv was flown through the device and allowed to interact with the biotin groups present on the SLB. Due to the increased hydrodynamic volume of SAv compared to TR-DHPE we observed migration of the SAv due to shear stress from bulk flow of $0.5 \mathrm{mM} \mathrm{F}_{\mathrm{c}} \mathrm{CH}_{2} \mathrm{OH}$, an effect not observed for TR-DHPE (Figure 2.4b). This phenomenon has recently been utilized for separation of SLB-bound SAv under flow conditions. ${ }^{18}$ As a consequence, lower flow speeds were adopted to minimize this effect while applying a potential, i.e. $10 \mu \mathrm{l} / \mathrm{min}$ instead of $100 \mu \mathrm{L} / \mathrm{min}$. The fluorescence micrographs before and after applying $1 \mathrm{~V}$ for 30 minutes with corresponding profile plots are presented in Figures 2.4c-d, respectively.

As can be observed from Figure 2.4c, continuous flowing of $0.5 \mathrm{mM}_{\mathrm{c}} \mathrm{CH}_{2} \mathrm{OH}$ and redox cycling does not affect binding of SAv to the SLB as illustrated by the continuous presence of the protein. 
a)
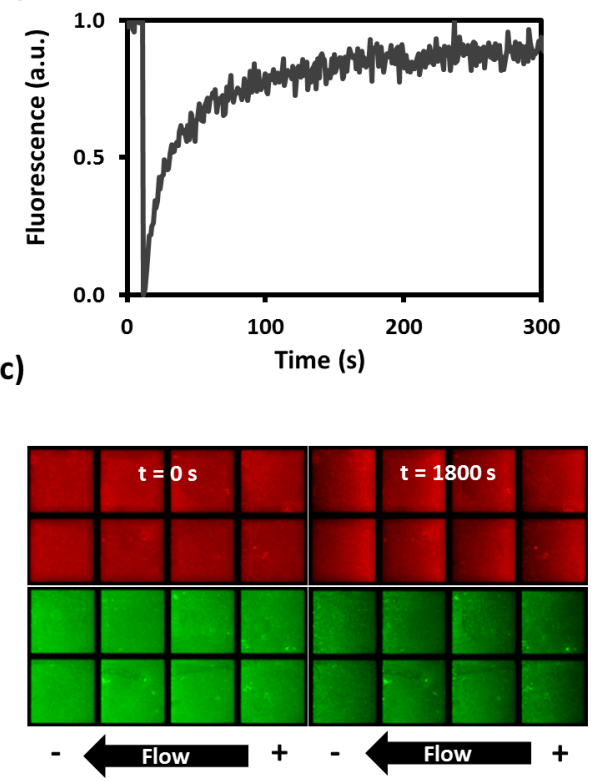

b)

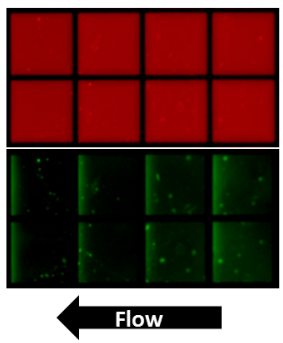

d)

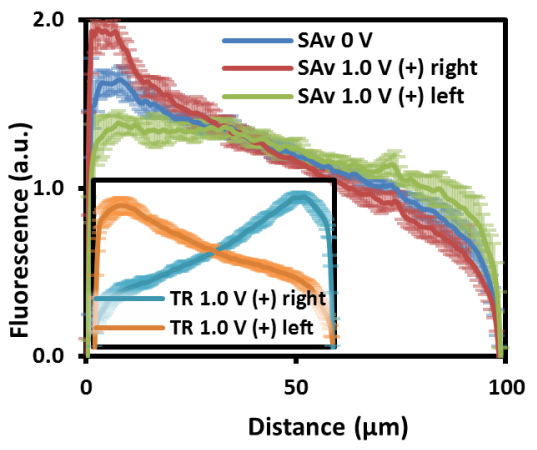

Figure 2.4: Streptavidin binding and redox cycling $0.5 \mathrm{mM} \mathrm{F}_{2} \underline{\mathrm{CH}}_{2} \mathrm{OH}_{;}$a) FRAP recovery curve of a biotinylated SLB. b) Fluorescence micrographs of SAv migration due to sheer stress from bulk flow within a SLB doped with 0.2 mol\% TR-DHPE (Red) and 1.0 mol\% biotinyl-PE after incubation with Alexa 488-labelled SAv (Green). c) Fluorescence micrographs of TR-DHPE and SAv-Alexa488 before and after applying $1.0 \mathrm{~V}$ for 30 minutes under continuous flow of $0.5 \mathrm{mM} \mathrm{F}_{\mathrm{c}} \mathrm{CH}_{2} \mathrm{OH}$. d) Graph showing the relative SAv-Alexa488 fluorescence intensity, before and after $1.0 \mathrm{~V}$ for 30 minutes and upon reversing the polarity of the electrodes. Inset shows the relative TR-DHPE fluorescence intensity as an internal control during the experiments. Data is presented as mean \pm SD.

The observation that ferrocene is not hampering the function of proteins is in agreement with literature where e.g. ferrocene moieties have been conjugated to proteins that remained functional irrespective of redox cycling. ${ }^{19,20}$ In addition, electrophoretic migration and switching of TR-DHPE was observed during the experiment (Figure 2.4d inset). Based on literature findings and the dye substitution ( $1.6 \mathrm{dyes} / \mathrm{molecule}$ ) of the SAv molecule, limited mobility towards the cathode can be expected. ${ }^{11,21}$ Figure $2.4 \mathrm{~d}$ illustrates that SAv build-up could be tuned by applying a potential of $1 \mathrm{~V}$ and altering the polarity of the electrodes even though the effect of flow was present. 
To evaluate the electrical parameters during the build-up of the uniform fluorescence profiles, Ohmic behaviour was assumed in between the electrodes as well as constant solution composition and conductivity. Applying Ohm's law, the electric field between two electrodes can be calculated as

$$
E=\frac{l}{3^{*} \sigma^{*} w^{*} h}
$$

where $E$ is the electric field in $\mathrm{V} / \mathrm{cm}, I$ the current in A, $\sigma$ the electrical conductivity in $\mathrm{S} / \mathrm{cm}$ $(4 \mu \mathrm{S} / \mathrm{cm}), w$ the active electrode width $(500 \mu \mathrm{m}), h$ the height in $\mathrm{m}$ of the solution above the SLB $(50 \mu \mathrm{m})$ and the factor 3 arises from the three junctions in parallel exposed to the solution when the flow channel is bonded to the chip, as shown in Figure 2.1a. ${ }^{22}$ Equation 1 evidently specifies two parameters that require attention i.e. the current and the conductivity of the solution. Increasing the concentration of $\mathrm{F}_{\mathrm{c}} \mathrm{CH}_{2} \mathrm{OH}$ up to the solubility limit of $0.5 \mathrm{mM}$, gave a huge increase in current at low applied potentials below the electrolysis potential of water, while the conductivity of the solution only increased slightly up to $4 \mu \mathrm{S} / \mathrm{cm}$ (see Figure 2.5a for a cyclic voltammogram, $\mathrm{CV}$, of $\mathrm{F}_{\mathrm{c}} \mathrm{CH}_{2} \mathrm{OH}$ ). The addition of flow over the chip increased currents by a factor of ca. 3 , while the current and thus the electric field were stabilized. As shown in Figure $2.5 \mathrm{~b}$, the current could be tuned by varying the applied potential and flow speed. There was no influence noted of the flow on SLB characteristics up to a flow speed of $0.3 \mathrm{~mL} / \mathrm{min}$, based on TR-DHPE observations.

Alternatively, the electric field can be deduced from the fluorescence profile at steadystate using a method given by Boxer and co-workers who described the steady-state concentration gradients to result from a competition between random diffusion and electric field-induced drift. ${ }^{7}$ By least squares fitting of the experimental steady-state concentration gradients to Equation 2, the electric field was obtained. In

$$
I(x)=A e^{-\frac{\mu z E x}{D}}
$$

$I(x)$ is the normalized fluorescence intensity at position $x$ (a.u.), $A$ is the maximum normalized fluorescence intensity (a.u.), $D$ the diffusion constant $\left(\mathrm{cm}^{2} / \mathrm{s}\right), \mu$ the electrophoretic mobility $\left(\mathrm{cm}^{2} /\left(\mathrm{V}^{*} \mathrm{~s}\right)\right)$ and $z$ the charge of the probe.

A representative fit to determine the electric field is shown in Figure 2.5c. The two aforementioned methods for deducing the electric field, by using Equation 1 or by fitting Equation 2, are compared in Figure $2.5 d$. The two methods favourably agree $\left(r^{2}=0.97\right)$. 
a)

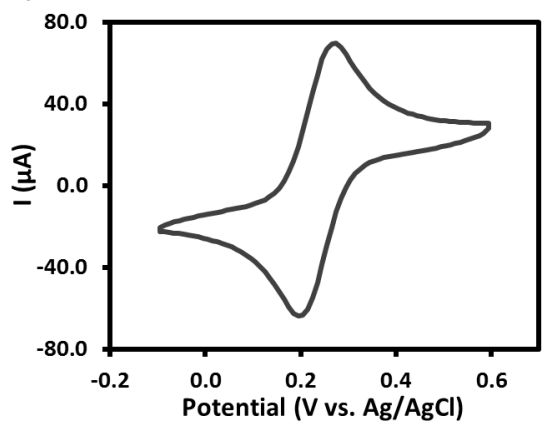

c)

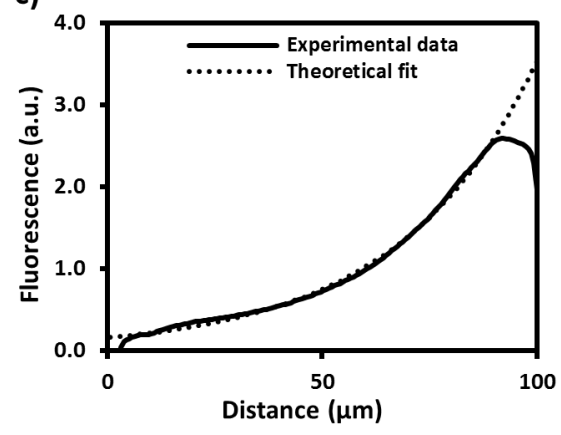

b)

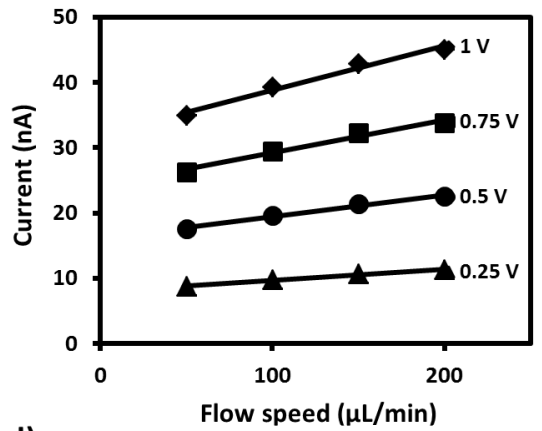

d)

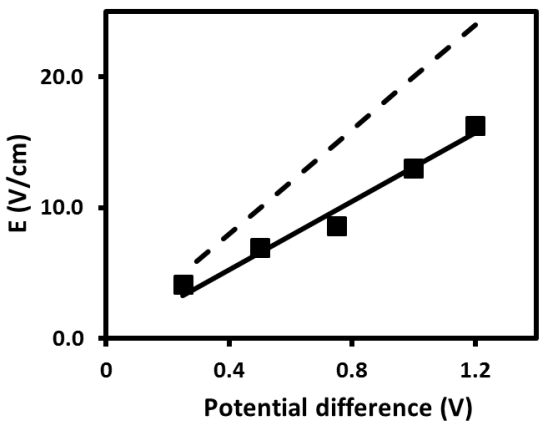

Figure 2.5: Electrical parameters; a) $\mathrm{CV}$ of $1.0 \mathrm{mM} \mathrm{F}_{\mathrm{c}} \mathrm{CH}_{2} \mathrm{OH}$ in $0.1 \mathrm{M} \mathrm{K}_{2} \mathrm{SO}_{4}$ vs. $\mathrm{Ag} / \mathrm{AgCl}$. b) The modulation of the current at different applied potentials and varying flow speeds in the presence of $0.5 \mathrm{mM} \mathrm{F}_{\mathrm{c}} \mathrm{CH}_{2} \mathrm{OH}$. c) Example of deducing the electric field by a theoretical fit of the exponential fluorescence intensity profile ( $1 \mathrm{~V}, 20$ minutes), see text for details. d) Comparing the applied electric field at $100 \mu \mathrm{L} / \mathrm{min}$ deduced from the current data in (b) (Solid line), exponential fit from (c) (Squares) and the geometrical approximation only (Dashed line).

The dashed line in Figure $2.5 \mathrm{~d}$ represents an electric field estimated by electrode distance only, often the method of choice in literature. ${ }^{7,12,14}$ As shown in Figure $2.5 \mathrm{~d}$ when utilizing the latter method, for example in our case at $1 \mathrm{~V}$, the electric fields can be overestimated by more than $50 \%$. This can be explained by the potential drops at both electrodes due to the reaction and concentration over-potential. As a result of the reduced distance between both electrodes, the contributions of these over-potentials of a few $100 \mathrm{~s}$ of millivolts at each electrode will be relatively large. Joule heating is negligible in our system as the total power dissipation of $2.6 * 10^{-5} \mathrm{~W} / \mathrm{cm}^{2}$ corresponds to an increase in temperature of only $0.13 \mathrm{mK} / \mathrm{s}$ without taking flow conditions into consideration. Moreover, the chip could be used for at least 7 cycles and stored for later use. 


\subsection{Conclusions}

A novel method to enable electrophoresis in micro supported lipid bilayers (SLB) has been demonstrated that occurs without the electrolysis of water through the addition of an sacrificial electro-active species. This is an important achievement because this allows for the use of unprecedented low potentials in $\mu$ SLB electrophoresis. Additionally, simple approximations accurately describe the processes at play during electrophoresis in the SLB. Since the direction of electrophoretic motion can be modulated, this technique is not only suitable for DC applications but also for AC surface ratchet applications. ${ }^{16,17}$ Our method is not limited to the use of $\mathrm{F}_{\mathrm{c}} \mathrm{CH}_{2} \mathrm{OH}$. Any water soluble electro-active species with similar voltammetric characteristics can potentially be used when inert to the SLB and analyte. Most noteworthy, the achieved results show no water electrolysis, negligible joule heating, no bubble formation, require only low flow speeds, and compatibility with biomolecules. These characteristics permit further downscaling of analytical devices. Therefore, we believe our method will be beneficial to further the field of SLB electrophoresis for bio-sensing, diagnostics and membrane protein studies, which require sorting and concentration of charged membrane components.

\subsection{Experimental section}

\subsubsection{Materials}

All starting materials and chemicals were purchased from Sigma-Aldrich, Fluka, Serva, Becton Dickinson, Avanti Polar Lipids, Microchem, Invitrogen and FujiFilm, and they were used as received, unless otherwise stated. MilliQ water with a resistivity higher than 18 $\mathrm{M} \Omega \mathrm{cm}^{-1}$ was used in all experiments.

\subsubsection{Methods}

Chip fabrication. A bilayer lift-off recipe was used for fabricating Au electrodes on borofloat glass wafers. First, LOR 5A (MicroChem) was spin-coated, after which normal lithography was performed on top with Olin OiR 907-17 photoresist (FujiFilm) to create a bilayer resist stack. Electrode patterns were made by exposing the photoresist through a patterned photomask and developing in Olin OPD 4262 (FujiFilm). The develop step washed away the exposed photoresist, and etching through the LOR 5A layer created an undercut. Then, $5 \mathrm{~nm} \mathrm{Ti}$ and $95 \mathrm{~nm}$ Au were deposited via e-beam evaporation (BAK 600, Balzers) The bilayer resist was then removed by sonication in acetone (20 minutes) and isopropanol (10 minutes) followed by 5 minutes immersion in OPD 4262, serving as a sacrificial layer to leave patterned Au electrodes on borofloat glass. To fabricate the $\mathrm{Cr}$ - 
corrals (10 $\mathrm{nm}$ thick) in between the Au electrodes, the same procedure was performed a second time, but in this case following alignment with respect to the Au electrodes.

PDMS flow channel. A silicon flow channel master was produced by standard photolithography steps and deep reactive ion etching. The polydimethylsiloxane (PDMS) flow channels were prepared from a degassed mixture of 10:1 Sylgard 184 elastomer and curing agent (Dow Corning Corp), which was casted onto the silicon master and cured at $80^{\circ} \mathrm{C}$ for 1 hour. The flow channels were cut to size and inlets and outlets were punched using a $1 \mathrm{~mm} \varnothing$ punch (Harris Unicore, Sigma-Aldrich).

Vesicle preparation. 1,2-Dioleoyl-sn-glycero-3-phosphocholine (DOPC, Avanti Polar Lipids) was stored as a $25 \mathrm{mg} / \mathrm{mL}$ stock solution in chloroform at $-20^{\circ} \mathrm{C}$. 1,2-Dioleoyl-sn-glycero-3phosphoethanolamine-N-biotinyl (biotinyl-PE, Avanti Polar Lipids) was stored as a 10 $\mathrm{mg} / \mathrm{mL}$ stock in chloroform at $-20^{\circ} \mathrm{C}$. The charged lipid-dye conjugate, Texas Red-1,2dihexadecanoyl-sn-glycero-3-phosphoethanolamine (TR-DHPE, Invitrogen) was stored as as $1 \mathrm{mg} / \mathrm{mL}$ stock solution in methanol at $-20^{\circ} \mathrm{C}$. Aliquots of the DOPC (99.8 mol\%) and TRDHPE (0.2 mol\%) or DOPC (98.8 mol\%), TR-DHPE (0.2 mol\%) and Biotinyl-PE ( $1 \mathrm{~mol})$ stock solutions were mixed and dried under a flow of nitrogen in a glass vial, and subsequently placed under vacuum for at least 1 hour. The resulting lipid film was re-suspended by vortexing in MilliQ water to form multilamellar vesicles (MLVs) at $1 \mathrm{mg} / \mathrm{mL}$. The MLV solution was extruded 11 times through a $100 \mathrm{~nm}$ polycarbonate membrane (Avanti Polar Lipids) at r.t. The resulting LUVs were characterized by DLS (Nanotrac, Microtrac) and stored until use at $4^{\circ} \mathrm{C}$ for a maximum of two weeks.

SLB formation. SLB formation was achieved by dilution of the LUV solution to $0.5 \mathrm{mg} / \mathrm{mL}$ in phosphate-buffered saline (0.01 M PBS, Gibco, lacking $\mathrm{MgCl}_{2}$ and $\mathrm{CaCl}_{2}$ ). Prior to $\mathrm{LUV}$ incubation, the chips were flushed briefly with 0.01 M PBS. Afterwards the chips were incubated with the vesicle suspension for a least 30 minutes to allow for vesicle adsorption and rupture to occur. Subsequently, the chips were washed with MilliQ water or aqueous $0.5 \mathrm{mM} \mathrm{F}_{\mathrm{c}} \mathrm{CH}_{2} \mathrm{OH}$.

Streptavidin. Alexa488-labelled streptavidin (Invitrogen) was dissolved in 0.01 M PBS and stored at $-20^{\circ} \mathrm{C}$ in $1 \mathrm{mg} / \mathrm{mL}$ aliquots until further use. Based on A280 and A494 absorption measurements (NanoDrop 1000, Thermo Scientific) 1.6 dye molecules per streptavidin was deduced. Biotinylated SLBs were incubated with $100 \mu \mathrm{g} / \mathrm{mL}$ for 15 minutes after that unbound streptavidin was washed away with MilliQ. 
Fluorescence Recovery After Photobleaching (FRAP). FRAP measurements were conducted using a Nikon A1 CSLM with a 20x objective. To derive the diffusion coefficient, modified Bessel functions as described by Soumpasis et al. 1983 were used. Data was corrected for acquisition bleaching and normalized.

$\mu S L B$ electrophoresis. Prior to SLB formation, wires were soldered onto the gold contact pads. Electric fields were generated and currents were measured with a $\mathrm{CH}$ Instruments 760D potentiostat with the counter and reference connections shorted. During $\mu \mathrm{SLB}$ electrophoresis, a freshly prepared solution of $0.5 \mathrm{mM}$ of $\mathrm{F}_{\mathrm{c}} \mathrm{CH}_{2} \mathrm{OH}$ in MilliQ water was flushed through the chip at $100 \mu \mathrm{L} /$ min unless otherwise stated.

Fluorescence microscopy. An Olympus inverted IX71 epi-fluorescence research microscope with a Xenon X-cite 120PC as light source and a digital Olympus DR70 camera for image acquisition was used to acquire fluorescence micrographs during $\mu \mathrm{SLB}$ electrophoresis at 10x magnification. Alexa488-labelled SAv was imaged using $460 \leq \lambda_{\mathrm{ex}} \leq$ $490 \mathrm{~nm}$ and $\lambda_{\mathrm{em}}>525 \mathrm{~nm}$, TR-DHPE was imaged using $510 \leq \lambda_{\mathrm{ex}} \leq 550 \mathrm{~nm}$ and $\lambda_{\mathrm{em}}>590$ $\mathrm{nm}$. ISO200 camera setting was used to record high quality, low noise images and care was taken to ensure image acquisition was performed in the linear response regime. Per corral the mean pixel intensity was used to correct the profile plot after which a background correction was performed. To retrieve the relative background, the steadystate fluorescence profiles at 1.2, 1.0 and $0.75 \mathrm{~V}$ were fitted to an exponential growth function, via the equation

$$
I(x)=I(0)(1+r)^{x}
$$

where $I(x)$ is the normalized fluorescence intensity at position $x, I(0)$ the background fluorescence intensity, $r$ the growth rate and $x$ the distance $(\mu \mathrm{m})$. Data fitting was conducted on the average profile plots deduced per column of corrals perpendicular to the electrodes. Subsequently, the background fraction (BF) of the mean pixel intensity was calculated. The background fractions of aforementioned conditions were used to calculate an average BF of $62 \pm 4 \%$, that was used to calculate and correct the background in all of the obtained profile plots, per corral. To obtain the final, averaged, steady-state fluorescence profiles at one potential, at least 14 normalized and background corrected corrals were averaged.

Data analysis. Image analysis was performed using ImageJ (NIH), Origin (OriginLab) and Excel (Microsoft). FRAP data was analysed with FRAPAnalyser (University of Luxembourg). 


\subsection{References}

1 Malinova, V.; Nallani, M.; Meier, W. P.; Sinner, E. K. FEBS Lett. 2012, 586, 2146.

2 Schiller, S. M. In Handbook of Biofunctional Surfaces, Chapter 18; Knoll, W., Ed.; Pan Stanford Publishing Pte. Ltd.: Singapore, 2013.

3 Tamm, L. K.; Mcconnell, H. M. Biophys. J. 1985, 47, 105-113.

$4 \quad$ Sackmann, E. Science 1996, 271, 43-48.

5 Salaita, K.; Nair, P. M.; Petit, R. S.; Neve, R. M.; Das, D.; Gray, J. W.; Groves, J. T. Science 2010, 327, 13801385.

$6 \quad$ Nair, P. M.; Salaita, K.; Petit, R. S.; Groves, J. T. Nat. Protoc. 2011, 6, 523-539.

7 Groves, J. T.; Boxer, S. G. Biophys. J. 1995, 69, 1972-1975.

8 Groves, J. T.; Boxer, S. G.; McConnel, H. M. Proc. Natl. Acad. Sci. USA 1997, 94, 13390-13395.

9 Groves, J. T.; Ulman, N.; Boxer, S. G. Science 1997, 275, 651-653.

10 Grogan, M. J.; Kaizuka, Y.; Conrad, R. M.; Groves, J. T.; Bertozzi, C. R. J. Am. Chem. Soc. 2005, 127, $14383-$ 14387.

Han, X.; Cheetham, M. R.; Sheikh, K.; Olmsted, P. D.; Bushby, R. J.; Evans, S. D. Integr. Biol. 2009, 1, 205211. Lee, Y. K.; Nam, J. M. Small 2012, 8, 832-837.

Pace, H. P.; Sherrod, S. D.; Monson, C. F.; Russell, D. H.; Cremer, P. S. Anal. Chem. 2013, 85, 6047-6052. Ammam, M.; Fransaer, J. Electroanal. 2011, 23, 755-763.

Monson, C. F.; Pace, H. P.; Liu, C.; Cremer, P. S. Anal. Chem. 2011, 83, 2090-2096.

Cheetham, M. R.; Bramble, J. P.; McMillan, D. G. G.; Krzeminski, L.; Han, X.; Johnson, B. R. G.; Bushby, R. J.; Olmsted, P. D.; Jeuken, L. J. C.; Marritt, S. J.; Butt, J. N.; Evans, S. D. J. Am. Chem. Soc. 2011, 133, 65216524.

Bao, P.; Cheetham, M. R.; Roth, J. S.; Blakeston, A. C.; Bushby, R. J.; Evans, S. D. Anal. Chem. 2012, 84, 10702-10707.

Hu, S. K.; Hsiao, S. W.; Mao, H. Y.; Chen, Y. M.; Chang, Y.; Chao, L. Sci. Technol. Adv. Mat. 2013, 14.

Yang, L. T.; Gomez-Casado, A.; Young, J. F.; Nguyen, H. D.; Cabanas-Danes, J.; Huskens, J.; Brunsveld, L.; Jonkheijm, P. J. Am. Chem. Soc. 2012, 134, 19199-19206. Wasserberg, D.; Uhlenheuer, D. A.; Neirynck, P.; Cabanas-Danes, J.; Schenkel, J. H.; Ravoo, B. J.; An, Q.; Huskens, J.; Milroy, L. G.; Brunsveld, L.; Jonkheijm, P. Int. J. Mol. Sci. 2013, 14, 4066-4080. Monson, C. F.; Pace, H. P.; Liu, C.; Cremer, P. S. Anal. Chem. 2011, 83, 2090-2096.

Bockris, J. O. M.; Reddy, A. K. N. Modern Electrochemistry second edition - Ionics; Kluwer Academic Publishers: New York, 2002; Vol. 1. 


\section{Chapter 3}

\section{Locked-in biomimetic surface gradients that are tuneable in size, density and functionalization ${ }^{*}$}

Tuneable and stable surface-chemical gradients in supported lipid bilayers (SLBs) hold great promise for the investigation and manipulation of membrane proteins, for sensing and study of the cell-cell and cell-extracellular matrix (ECM) interface. Yet, until now no method has been reported that provides temporal control of SLB gradients. Herein, we report on the development of locked-in SLB gradients that can be tuned in space, time and density by applying a process to control lipid phase behaviour, electric field and temperature. Stable gradients of charged Texas Red, serine or biotin terminated lipids are prepared. For example, the Texas Red surface density was varied from 0 to $2 \mathrm{~mol} \%$, while the length is varied between several tens to several hundreds of microns. At room temperature the gradients are shown to be stable up to $24 \mathrm{~h}$, while at $60^{\circ} \mathrm{C}$ the gradients could be erased in 30 minutes. Covalent and non-covalent chemical modification of the gradients is demonstrated e.g. by FITC, hexahistidine-tagged proteins and streptavidin/biotin. The amenability to various (bio)chemistries paves the way for novel SLB-based gradients, useful in sensing, high-throughput screening and for understanding dynamic biological processes.

\footnotetext{
${ }^{*}$ Part of this chapter has been published in: S.O. Krabbenborg ${ }^{\ddagger}$, J. van Weerd ${ }^{\ddagger}$, M. Karperien, P. Jonkheijm and J. Huskens; ChemPhysChem (2014) vol. 15, p. $3460-3465$ (' equal authorship)
} 


\subsection{Introduction}

In biology the influence of gradients on cell behaviour, both surface-bound (haptotaxis), ${ }^{1-3}$ and in solution (chemotaxis), ${ }^{4-6}$ has gained great attention. Man-made surface chemical gradients have been used to study parameters influencing cell adhesion, migration, morphology and downstream signalling processes, ${ }^{7-11}$ but also in materials science, ${ }^{12}$ such as biomaterials ${ }^{11}$ or sensing materials. ${ }^{13}$

Gradients in wettability, of extracellular matrix (ECM) proteins, peptide motifs or signalling molecules can be prepared by adsorption or covalent modification on a range of surfaces, such as self-assembled monolayers (SAMs), polymer layers/grafts, and hydrogels. ${ }^{7,10,11,14-16}$ An unexplored, yet very interesting platform for gradient formation is a supported lipid bilayer (SLB), as they have several advantages over the present systems. SLBs are well described with respect to their formation, non-fouling behaviour, and functionalization. ${ }^{17}$ This rewarding biomimetic cell membrane system ${ }^{18,19}$ promotes investigation of cell membrane properties and membrane associated processes. Membrane proteins can readily be reconstituted in SLBs in analogy to their native membranous environment. ${ }^{18-23}$ Furthermore, SLBs have been used extensively to explore cell-cell and cell-ECM interactions. $^{22,24-28}$ Various studies showed a strong density dependence of SLB ligands on cells in steady-state systems. ${ }^{29,30}$ However, such non-gradient systems do not allow for spatial and temporal control of the membrane composition, which is essential for understanding dynamic biological processes.

As was shown in Chapter 2, formation of gradients in SLBs can be achieved upon application of an electric field. ${ }^{31-35}$ The fluidic nature of the SLBs is thereby exploited to induce electrophoretic or electro-osmotic migration of charged membrane components. In one study such gradients are used as a sensing element by studying the time needed for gradient build-up. ${ }^{34}$ In addition, SLB electrophoresis has been adopted to separate and study membrane components via mass spectroscopy. ${ }^{36}$ A characteristic feature of the aforementioned systems is the use of liquid-state SLBs that readily relax back to their equilibrium position as soon as the electric field is turned off. This effect can be retarded by topographically restricting lateral diffusion by introduction of surface ratchets. ${ }^{37}$ Still, the lipids in these systems display lateral mobility that restrict the temporal stability of the surface gradients. This lack of temporal control limits the use of SLB platforms for studying biological processes.

In this Chapter, we demonstrate functional SLB gradients that are stable over time by the use of gel-state lipids. Gradient stability is imparted by the use of zwitterionic gel-state SLBs that display negligible mobility at room temperature $\left(<10^{-3} \mu \mathrm{m}^{2} / \mathrm{s}\right)^{38}$ because of 
saturated acyl tails which pack more tightly, resulting in an increased melting temperature $\left(T_{m}\right)$. Functionalities are introduced via charged lipids that allow for subsequent modification. To this end, we investigated a variety of chemical strategies, exploring both covalent and non-covalent approaches to generate locked-in and functional SLB gradients.

\subsection{Results and discussion}

The working principle of generating locked-in SLB gradients is shown in Figure 3.1. The SLB gradients are formed electrochemically, as described in Chapter $2 .^{35}$

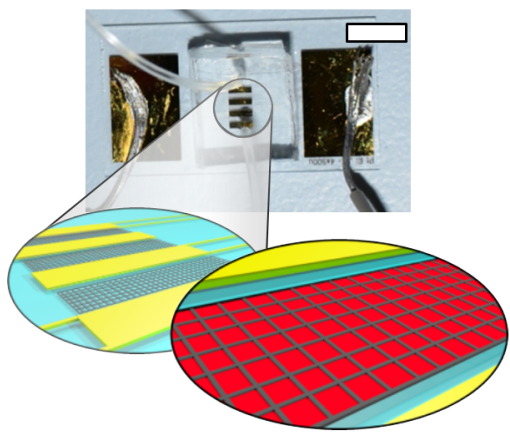

Steady-state SLB

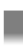

Electrophoresis and heating, $T>T_{m}$

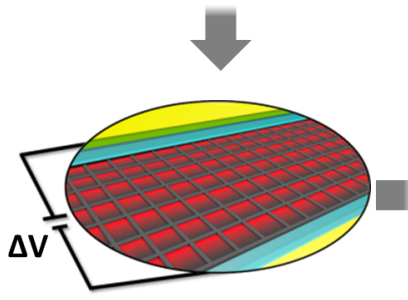

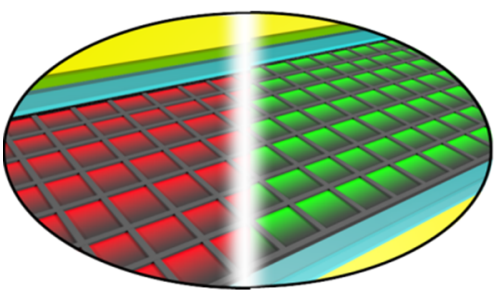
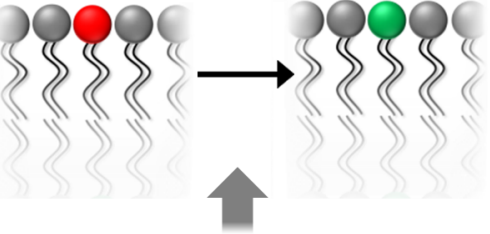

Functionalization

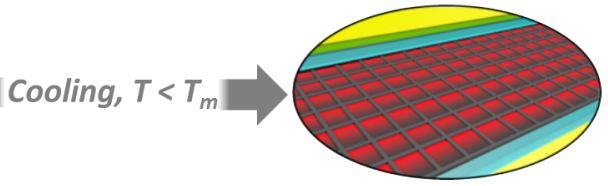

Locked-in gradient

Figure 3.1: Working principle of locked-in SLB gradients; the $\mu$ SLB electrophoresis device (scale bar 5 $\mathrm{mm})$ is modified with a zwitterionic DPPC-SLB $(Z=0)$ doped with a charged functional lipid $(Z \neq 0)$ using vesicle fusion. Subsequently, the device is heated above the $T_{m}$ and $1 \mathrm{~V}$ is applied for 30 minutes in the presence of $0.5 \mathrm{mM} \mathrm{F}_{\mathrm{c}} \mathrm{CH}_{2} \mathrm{OH}$, thereby generating an E-field. Due to electrophoretic migration, the charged functional lipids accumulate and are locked-in upon rapid cooling below the $\mathrm{T}_{\mathrm{m}}$. The locked-in gradient can be modified using a variety of chemistries to create functional gradients. 
A zwitterionic SLB consisting of DPPC, doped with negatively charged and fluorescent TRDHPE is formed on the device by fusion of the large unilaminar vesicles (LUVS). The chemical structure of DPPC and the dynamic light scattering (DLS) measurement of DPPC LUVs are shown in Figure 3.2a and 3.2b, respectively. Since DPPC has a $T_{m}$ of $41^{\circ} \mathrm{C}$, no mobility of TR-DHPE was observed at room temperature using fluorescence recovery after photobleaching (FRAP) (Figure 3.2c). The lack of lateral mobility at room temperature is further evidenced over a time period of 15 hours, as shown in Figure 3.2d-e.

a)

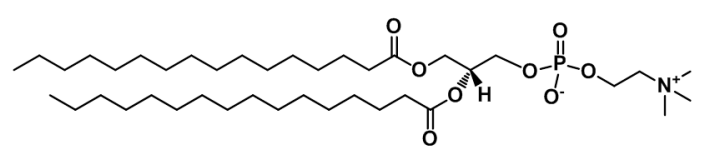

b)
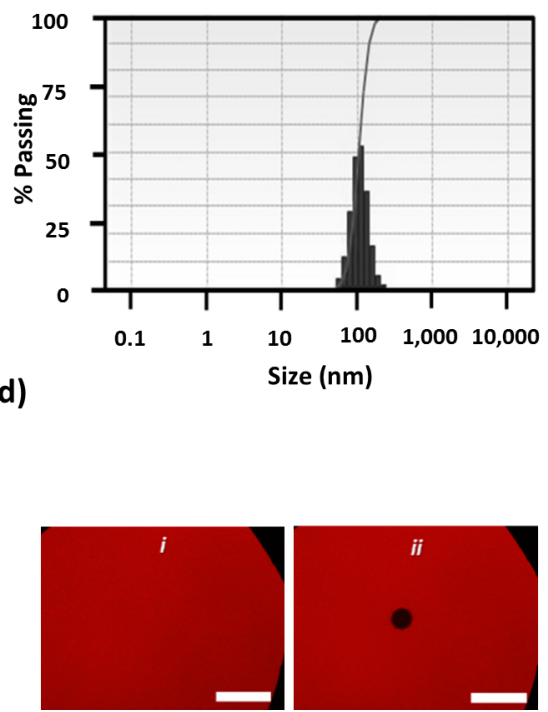

c)

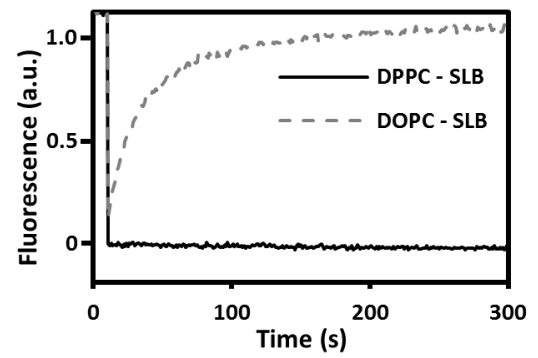

e)

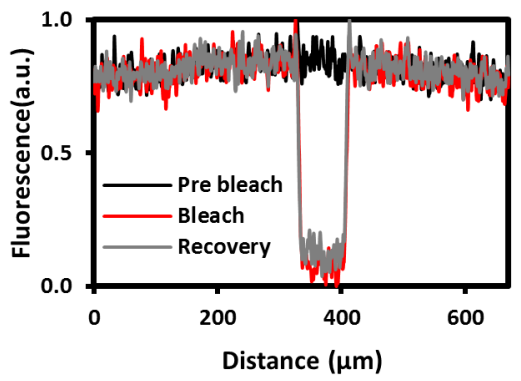

Figure 3.2: DPPC characteristics; a) chemical structure of DPPC, b) DLS measurement of DPPC LUVs doped with 0.2 mol\% TR-DHPE at room temperature, c) FRAP of a liquid-state (DOPC, $\mathrm{T}_{\mathrm{m}}-20^{\circ} \mathrm{C}$, Red) and a gel-state (DPPC, $\mathrm{T}_{\mathrm{m}} 41^{\circ} \mathrm{C}$, Black) SLB doped with $0.2 \mathrm{~mol} \%$ TR-DHPE conducted at room temperature. d) Representative fluorescent micrographs before, $i$, and 15 hours, ii, after bleaching and e) corresponding profile plots, of a DPPC- and DOPC-based SLB doped with 0.2 mol\% TR-DHPE. Negligible mobility of TR-DHPE was observed after bleaching with an epi-fluorescent microscope, as indicated by the overlap of the profile plot directly after bleaching (Bleach) and 15 hours after bleaching (Recovery), scale bar $200 \mu \mathrm{m}$. 
By electrochemically generating an electric field, a force was applied on the charged components within the SLB, in this case TR-DHPE. At room temperature, the gel-state DPPC-based SLB, which shows homogeneous fluorescence as was observed via epifluorescence microscopy (Figure 3.3a-i and 3.3c), prohibits electrophoretic mobility of TRDHPE. However, when the device was heated above the $T_{m}$ (here $60^{\circ} \mathrm{C}$ ) during electrophoresis, lateral diffusion and electrophoretic mobility became apparent (Figure 3.3a-ii). At that temperature, the now liquid-state SLB allowed TR-DHPE to form an exponential concentration profile against the $\mathrm{Cr}$-barriers induced by electrophoretic migration, similar to what has been observed previously, with the direction being dependent on electrode polarity (Figure 3.3b inset). Rapid cooling of the DPPC-based SLB below the $T_{m}$, still during electrophoresis, resulted in a liquid-to-gel phase transition, locking the gradient in time (Figure 3.3a-ii and 3.3c).

a)

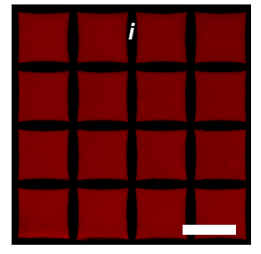

b)

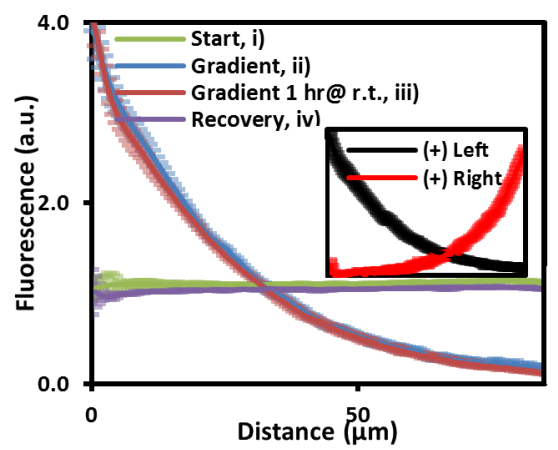

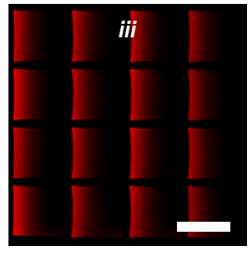

c)

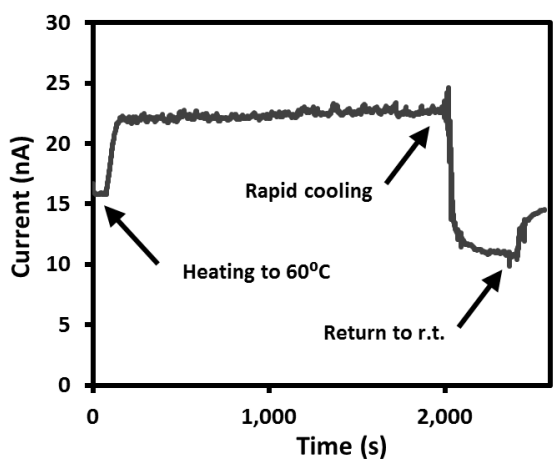

Figure 3.3: Locked-in SLB gradients; a) epi-fluorescence micrographs of $i$ steady-state DPPC-SLB doped with fluorescent and negatively charged TR-DHPE, ii locked-in gradient directly after heating, electrophoresis and rapid cooling to r.t. and iii after maintaining the sample at r.t. for 1 hour. iv. The locked-in gradient can be erased after heating the device for 30 minutes at $60^{\circ} \mathrm{C}$ showing recovery to the steady-state. Scale bar $100 \mu \mathrm{m}$. b) Profile plots of micrographs i, ii, iii and iv in Figure 3.3a. The inset shows a change in direction of electrophoretic mobility depending on electrode polarity. Data is presented as mean \pm SD. c) Graph showing the current as a function of time while generating functional gradients in SLBs. The different temperature steps can be discerned. 
All temperature variations were monitored via in situ current measurements (Figure 3.3c). The locked-in gradients in Figure 3.3 corroborate with our previous findings obtained with TR-DHPE doped DOPC-based SLBs described in Chapter 2. ${ }^{35}$ Temporal stability of the locked-in gradients was shown for at least 1 hour at room temperature (Figure 3.3a-iii), no deviation between the profile plots was observed (Figure 3.3c-ii \& iii). In the liquid-state however, it only takes a few minutes to relax the build-up and the steady-state can be reached within 30 minutes due to recovery and mobility of the lipids. This relaxation was also observed for locked-in TR-DHPE gradients that were heated to $60^{\circ} \mathrm{C}$ for 30 minutes in the absence of an electric field (Figure 3.3a-iv and 3.3c). These results demonstrate the reversibility of the system, i.e. the gradient can be erased by simply heating the system above the $T_{m}$.

By changing the length of the corral, the absolute amount of enclosed TR-DHPE can be varied. To this end, we fabricated an alternate chip with varying corral lengths. Figure $3.4 \mathrm{a}$ shows a fluorescence micrograph of a sample that was stored at room temperature for 24 hours after gradient formation, showing corral lengths of 100, 210 and $430 \mu \mathrm{m}$. In Figure $3.4 \mathrm{~b}$ the corresponding fluorescence intensity profiles are presented, all exhibiting similar exponential gradient profiles (Figure 3.4c). Figure 3.4d shows that the full widths at half maximum (FWHM) are similar, while Figure 3.4e shows that the peak height changes linearly with the corral length. These results are agreements with experiments when charge-charge interactions between charged lipids are absent. The results illustrate that the extent of build-up is dependent on and can be tuned with the corral length. The fluorescence intensity is normalized such that 1 a.u. corresponds to a TR-DHPE doping density of $0.2 \mathrm{~mol} \%$. As a result, changing the length of the corral, the maximal density of TR-DHPE could be varied from $\sim 0.6 \mathrm{~mol} \%$ to $2 \mathrm{~mol} \%$. This provides great tuneability and shows that the desired density gradient of a functional lipid can be optimized by corral length and doping density. Build-up would eventually be affected by charge-charge interactions between charged lipids, which is expected to occur when sufficiently high mol\% build-up is obtained, ${ }^{32}$ which would flatten out the gradient. 
a)

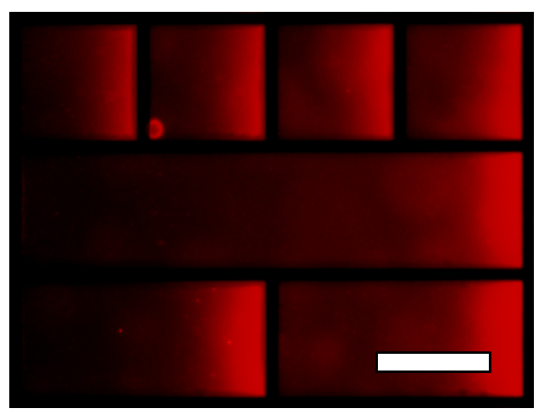

c)

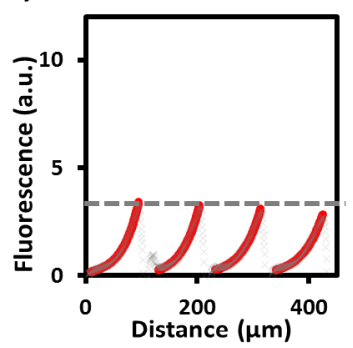

d)

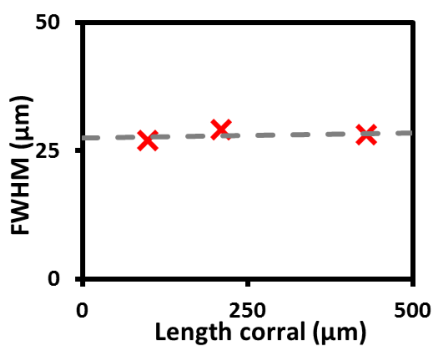

b)
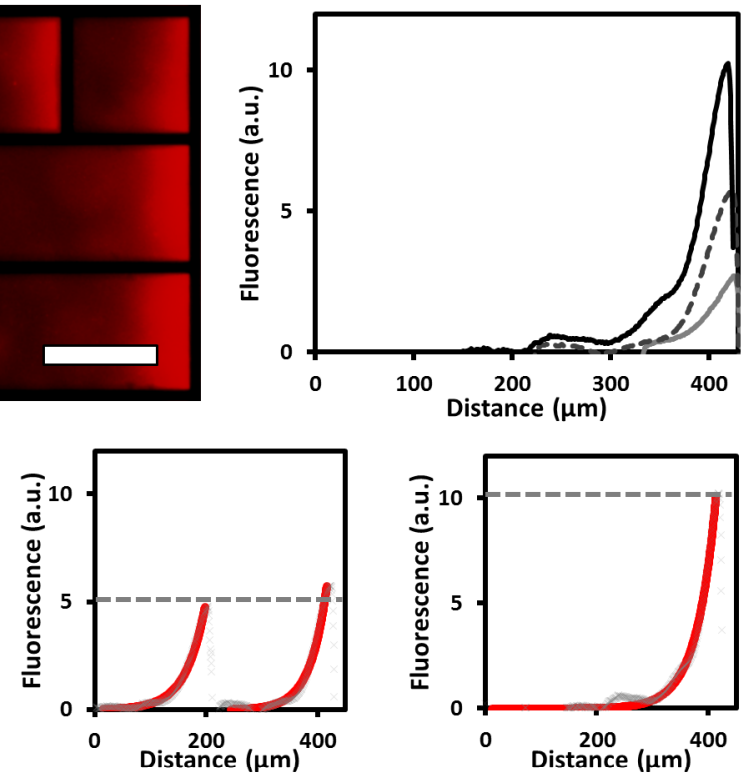

e)

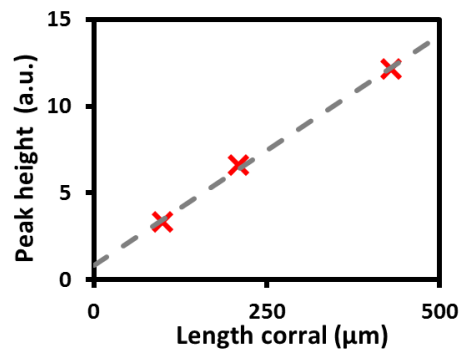

Figure 3.4: Effect of corral length; a) fluorescence micrograph obtained 24 hours after TR-DHPE gradient formation using varying corral dimensions and thus varying enclosed amounts of TR-DHPE. Scale bar $100 \mu \mathrm{m}$. b) Combined fluorescence intensity profiles of three different corral lengths and c) fluorescence intensity profiles per corral length, including theoretical exponential fits (Red). d) The full width half maximum plotted vs. corral length and e) peak height vs. corral length.

The first example of a functional lipid gradient is presented in Figure 3.5. Here, the zwitterionic DPPC-based SLB is doped with $1 \mathrm{~mol} \%$ of negatively charged serine-modified lipid (DOPS) bearing both an amine and a carboxylic acid group (Figure 3.5a). Adopting the previous approach, a DOPS gradient within a DPPC-SLB was prepared (Figure 3.5b-c). To visualize the gradient, the amine functionalities of the locked-in gradient were used to react with FITC, which enables fluorescent read-out of the DOPS gradient as shown in Figure 3.5c. Due to physisorption of the FITC to the PDMS flow channel, these gradients 
were imaged with confocal microscopy. An interesting thing to note is that when assuming quantitative modification with FITC, the fluorescence gradient is essentially a measure for carboxylic acid groups that are present at the gradient.

a)

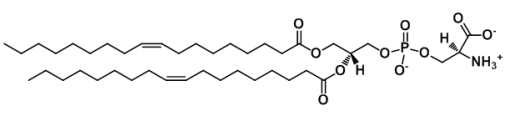

c)

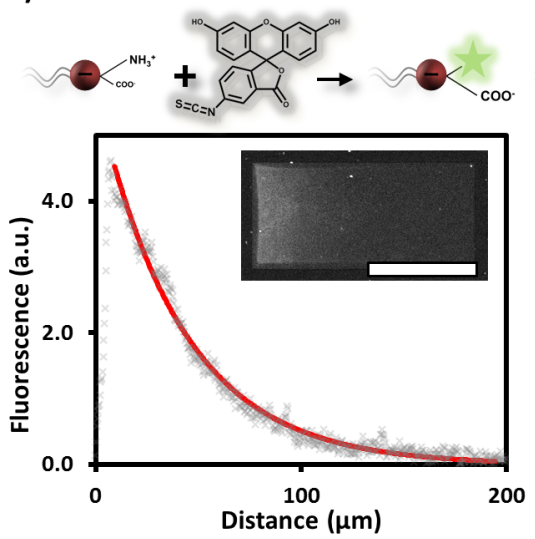

b)

d)
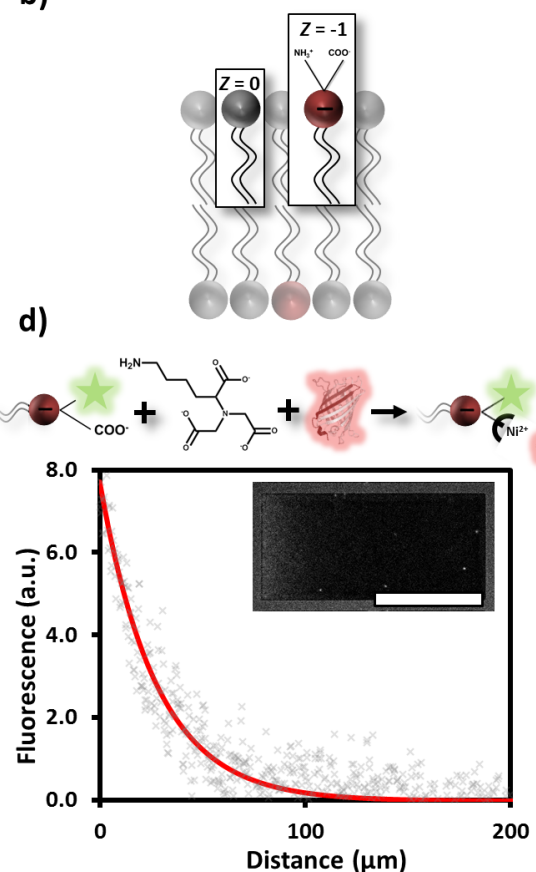

Figure 3.5: Amine and carboxylic acid SLB gradient; a) chemical structure of the DOPS lipid and b) schematic overview of the DPPC-SLB $(Z=0)$ doped with DOPS $(Z=-1)$, bearing an amine and carboxylic acid functionality. c) Amine-containing gradient reacted with FITC imaged using a confocal microscope. Corresponding fluorescence intensity profiles of the resulting fluorescent gradients, scale bar $100 \mu \mathrm{m}$. d) NTA functionalization of the remaining carboxylic acid-containing lipid gradient that are used to sequentially chelate and immobilize a fluorescent His-tagged protein. A representative confocal microscopy image is shown with corresponding fluorescence intensity profile of the resulting protein gradient, scale bar $100 \mu \mathrm{m}$.

As a next step, we attempted to further modify these available carboxylic groups (Figure $3.5 \mathrm{~d})$. To this end, first the carboxylic acid groups were activated with $N$ hydroxysuccinimide (NHS) and ethyl(dimethylaminopropyl) carbodiimide (EDC). Then, the NHS-activated gradient was allowed to react with an amine-terminated nitrilotriacetic acid (NTA) group. Rinsing the chip with a $\mathrm{NiCl}_{2}$ solution allowed $\mathrm{Ni}^{2+}$ metal ions to coordinate with the immobilized NTA and to chelate a fluorescent His-tagged protein, TagRFP. The representative confocal microscopy image of the resulting TagRFP protein gradient is given in Figure $3.5 \mathrm{~d}$, alongside with the exponential fluorescence intensity profile. Though 
the two FITC and TagRFP fluorescence profiles can not be compared quantitatively, presumably because of the multivalent binding of TagRFP to the gradient, the successful functionalization of the serine-modified lipid via the two different methods demonstrates the tuneability of locked-in gradients with respect to post-functionalization.

In a second example of fabricating a functional SLB gradient (Figure 3.6), DOPS was exchanged for a charged biotinylated lipid (biotinyl-PE). The zwitterionic DPPC-SLB was doped with 1 mol\% of negatively charged biotinyl-PE lipid (Figure 3.6a). After gradient formation using the aforementioned method, the biotin gradient was visualized by incubation with fluorescently labelled streptavidin (Alexa488-labelled SAv, green). The biotin-SAv gradient, as indicated by the Alexa488-labelled SAv fluorescence, is shown in Figure 4b. Upon incubation of the streptavidin gradient with fluorescently labelled biotin (Atto 565 conjugate, red) an additional biotin gradient was generated with an exponential concentration profile (Figure 4c), similar to the streptavidin gradient.

a)

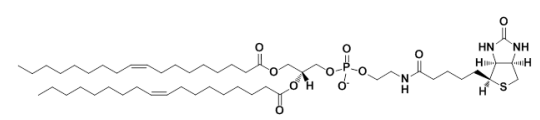

c)

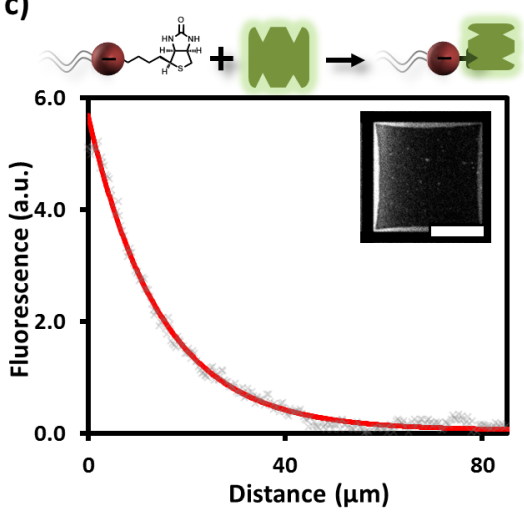

b)

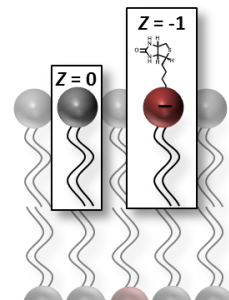

d)

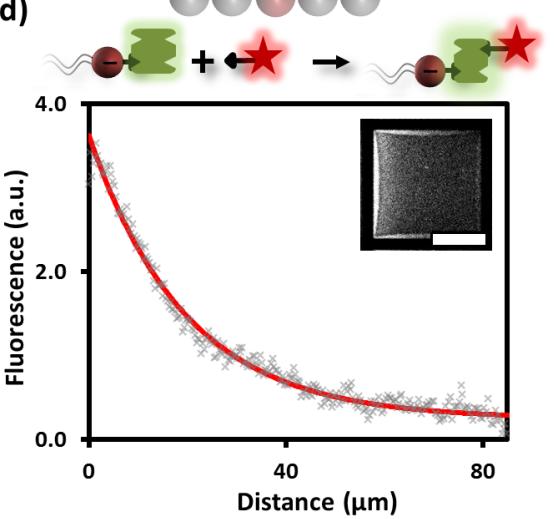

Figure 3.6: Biotin and streptavidin SLB gradient; a) chemical structure of biotinyl-PE and b) schematic overview of the DPPC-SLB $(Z=0)$ doped with the functional biotinylated lipid $(Z=-1)$. c) Schematic representation of the immobilization of fluorescently labelled SAv (Green) on the biotincontaining lipid gradient and a representative confocal microscopy image with corresponding fluorescence intensity profile of the resulting SAv protein gradient, scale bar $50 \mu \mathrm{m}$. d) Subsequent association of fluorescently labelled biotin (Red) on the SAv gradient with representative confocal microscopy image and fluorescence intensity profile of the resulting fluorescent biotin gradient, scale bar $50 \mu \mathrm{m}$. 


\subsection{Conclusions}

In conclusion, a novel method has been demonstrated for the preparation of locked-in SLB gradients that can be tuned in space, time and density in a process controlling lipid phase behaviour, electric field and temperature. In this Chapter, gradients of TR-DHPE, DOPS and biotinyl-PE lipids were shown. Covalent and non-covalent chemical modification was performed to form gradients of FITC, His-tagged proteins and SAv/biotin. This demonstrates that the proposed methodology is amenable to a wide range of relevant biochemistries. This important achievement allows for the further development of SLBbased applications in the areas of sensing, high-throughput screening and for understanding dynamic biological processes.

\subsection{Acknowledgements}

S. Sankaran, Dr. D. Wasserberg and Dr. J. Cabanas-Danés are greatly acknowledged for providing the TagRFP protein and NTA-NH , respectively.

\subsection{Experimental section}

\subsubsection{Materials}

All starting materials and chemicals were purchased from Sigma-Aldrich, Fluka, Serva, Becton Dickinson, Avanti Polar Lipids, Microchem, Invitrogen and FujiFilm and they were used as received, unless otherwise stated. MilliQ water with a resistivity of $18.2 \mathrm{M} \Omega \mathrm{cm}^{-1}$ was used in all experiments.

\subsubsection{Methods}

Chip fabrication, PDMS flow channel and PDMS bonding. For more information consult experimental section of Chapter 2. Additional care was taken to use fresh $\mathrm{F}_{c} \mathrm{CH}_{2} \mathrm{OH}$ solutions for gradient formation of the DOPS lipids. Thereby ensuring a near $\mathrm{pH}$-neutral of the $\mathrm{F}_{\mathrm{c}} \mathrm{CH}_{2} \mathrm{OH}$ solution.

Vesicle preparation. 1,2-Dipalmitoyl-sn-glycero-3-phosphocholine (DPPC, Avanti Polar Lipids) was stored as a $25 \mathrm{mg} / \mathrm{mL}$ stock solution in chloroform at $-20^{\circ} \mathrm{C}$. 1,2-Dioleoyl-snglycero-3-phosphoethanolamine-N-biotinyl (Biotinyl-PE, Avanti Polar Lipids) and 1,2dioleoyl-sn-glycero-3-phospho-L-serine (DOPS, Avanti Polar Lipids) were stored as a 10 $\mathrm{mg} / \mathrm{mL}$ stock in chloroform at $-20^{\circ} \mathrm{C}$. The charged lipid-dye conjugate, Texas Red-1,2dihexadecanoyl-sn-glycero-3-phosphoethanolamine (TR-DHPE, Invitrogen) was stored as a $1 \mathrm{mg} / \mathrm{mL}$ stock solution in methanol at $-20^{\circ} \mathrm{C}$. Desired molar ratios were mixed and dried 
under a flow of nitrogen in a glass vial, and subsequently placed under vacuum for at least 1 hour. The resulting lipid film was re-suspended by vortexing in MilliQ water to form multilamellar vesicles (MLVs) at $1 \mathrm{mg} / \mathrm{mL}$ at $60^{\circ} \mathrm{C}$. The MLV solution was extruded 11 times through a $100 \mathrm{~nm}$ polycarbonate membrane (Avanti Polar Lipids) at $60^{\circ} \mathrm{C}$. The resulting LUVs were characterized by dynamic light scattering (DLS, Nanotrac, Microtrac) and stored until use at r.t. for a maximum of two weeks.

SLB formation. Prior to SLB formation, wires were soldered onto the gold contact pads and the device was mounted onto a heat plate (IKA RCT classic) using scotch tape. SLB formation was achieved by dilution of the LUV solution to $0.5 \mathrm{mg} / \mathrm{mL}$ in phosphatebuffered saline (0.01 M PBS pH 7.4, Gibco, lacking $\mathrm{MgCl}_{2}$ and $\mathrm{CaCl}_{2}$ ). Prior to LUV incubation, the chips were flushed briefly with $0.01 \mathrm{M} \mathrm{PBS}$ and heated till $60^{\circ} \mathrm{C}$. Once heated, the chips were incubated with the vesicle suspension for a least 30 minutes to allow for vesicle adsorption and rupture to occur. Subsequently, the chips were washed with MilliQ water (300 $\mathrm{LL} / \mathrm{min})$ and allowed to cool down. Once at room temperature, aqueous $0.5 \mathrm{mM} \mathrm{F}_{\mathrm{c}} \mathrm{CH}_{2} \mathrm{OH}$ (Sigma-Aldrich) was flown through the device at the same flow speed.

Gradient formation via electrophoresis. Electric fields were generated and currents were measured with a $\mathrm{CH}$ Instruments 760D potentiostat with the counter and reference connections shorted. During $\mu \mathrm{SLB}$ electrophoresis, a freshly prepared solution of $0.5 \mathrm{mM}$ of $\mathrm{F}_{\mathrm{C}} \mathrm{CH}_{2} \mathrm{OH}$ in MilliQ water was flushed through the chip at $100 \mu \mathrm{L} / \mathrm{min}^{35}$ Prior to heating the device a potential difference of $1 \mathrm{~V}$ was applied for at least 30 seconds at r.t. Afterwards the chip was heated to $60^{\circ} \mathrm{C}$ and the potential difference of $1 \mathrm{~V}$ was applied for 30 minutes. Subsequently, the chip was cooled down rapidly on a heat exchanger for 5 minutes to lock-in the gradient.

FITC gradient. To generate a gradient consisting of fluorescein, the serine-bearing DOPS lipid was used as the functional lipid. After gradient formation the chip was flushed with freshly prepared 0.1 M sodium bicarbonate buffer (Sigma-Aldrich) at a pH of 9.0, adjusted with $\mathrm{NaOH}$. Subsequently, $50 \mu \mathrm{g} / \mathrm{mL}$ of fluorescein isothiocyanate isomer I (FITC, SigmaAldrich), dissolved in $0.1 \mathrm{M}$ sodium bicarbonate buffer $\mathrm{pH}$ 9.0, was flown through the device at $100 \mu \mathrm{L} / \mathrm{min}$ for 1 hour. ${ }^{39,40}$ After the incubation period the chips were extensively washed with $0.1 \mathrm{M}$ sodium bicarbonate buffer $\mathrm{pH}$ 9.0. The FITC gradients were imaged in PBS buffer, $\mathrm{pH} 7.4$.

NTA gradient. Preparation of NTA-NH $\mathrm{NH}_{2}$ is described elsewhere and was kindly provided by Dr. J. Cabanas. ${ }^{41,42}$ The acid groups of DOPS gradient were activated with $50 \mathrm{mM}$ of NHS (Sigma-Aldrich) and $50 \mathrm{mM}$ EDC (Sigma-Aldrich) $^{43}$ in $0.1 \quad \mathrm{M} \quad$ 2-(Nmorpholino)ethanesulfonic acid (MES buffer pH 5.0, Sigma-Aldrich) ${ }^{44,45}$ during a 1 hour 
incubation at r.t., $100 \mu \mathrm{L} / \mathrm{min}$ stop-flow. The device was flushed with 20:1 v/v 0.01 M PBS ( $\mathrm{pH} 7.4$ ) and $0.2 \mathrm{M}$ sodium bicarbonate solution ( $\mathrm{pH} 9.0$ ) at $100 \mu \mathrm{L} / \mathrm{min}$ (as indicated by Atto-Tec $\mathrm{GmbH}$ ), the resulting coupling buffer had a pH of 8.3 . Subsequently $50 \mathrm{mg} / \mathrm{mL}$ of NTA-NH $\mathrm{N}_{2}$ in coupling buffer was allowed to react for 1 hour, $100 \mu \mathrm{L} / \mathrm{min}$ stop-flow. The device was flushed with PBS at $100 \mu \mathrm{L} / \mathrm{min}$ to remove reactants. A $40 \mathrm{mM} \mathrm{NiCl}$ solution was flown through the device for 30 minutes to coordinate $\mathrm{Ni}^{2+}$ ions with the NTA-groups, $100 \mu \mathrm{L} / \mathrm{min}$ stop-flow. After washing the device with PBS, $1 \mu \mathrm{M}$ of hexahistidine-tagged TagRFP, provided by S. Sankaran and Dr. D. Wasserberg, ${ }^{46}$ in PBS was added at $10 \mu \mathrm{L} / \mathrm{min}$ for 30 minutes. After a final wash with PBS at $10 \mu \mathrm{L} / \mathrm{min}$, the device was imaged with confocal microscopy.

Streptavidin-biotin gradient. Alexa fluor 488 labelled streptavidin (Invitrogen) was dissolved in $0.01 \mathrm{M} \mathrm{PBS}$, and stored at $-20^{\circ} \mathrm{C}$ in aliquots until further use. Based on A280 and A494 absorption measurements (NanoDrop 1000, Thermo Scientific) 1.6 dye molecules per streptavidin was deduced. Gradients of 1 mol\% Biotinyl-PE were incubated with $50 \mu \mathrm{g} / \mathrm{mL}$ of labelled streptavidin in PBS for 30 minutes after which the unbound streptavidin was washed away with $0.01 \mathrm{M}$ PBS buffer and the gradients were imaged, flow speeds of $10 \mu \mathrm{L} / \mathrm{min}$ were used. Labelling of immobilized streptavidin was achieved with biotin Atto 565 (Fluka). Here, $50 \mu \mathrm{g} / \mathrm{mL}$ in PBS was flown through the chip at 10 $\mu \mathrm{L} / \mathrm{min}$ and left to interact for 30 minutes. Afterwards the chips were washed with PBS buffer at $10 \mu \mathrm{L} / \mathrm{min}$ and imaged.

Fluorescence microscopy. An Olympus inverted IX71 epi-fluorescence research microscope with a Xenon X-cite 120PC light source and a digital Olympus DR70 camera was used to acquire fluorescence images at 10x magnification. TR-DHPE was imaged using $510 \leq \lambda_{\mathrm{ex}} \leq 550 \mathrm{~nm}$ and $\lambda_{\mathrm{em}}>590 \mathrm{~nm}$. ISO200 camera setting was used to record high quality, low noise images and care was taken to ensure image acquisition was performed in the linear response regime. Confocal image acquisition was performed with a Nikon A1 CSLM with a 20x objective. Image treatment was performed similarly to the method described in Chapter 2.

Data analysis. Image analysis was performed using ImageJ (NIH), Origin (OriginLab) and Excel (Microsoft). 


\subsection{References}

Baier, H.; Bonhoeffer, F. Science 1992, 255, 472-475.

Wu, C.; Asokan, S. B.; Berginski, M. E.; Haynes, E. M.; Sharpless, N. E.; Griffith, J. D.; Gomez, S. M.; Bear, J. E. Cell 2012, 148, 973-987.

Weber, M.; Hauschild, R.; Schwarz, J.; Moussion, C.; de Vries, I.; Legler, D. F.; Luther, S. A.; Bollenbach, T.; Sixt, M. Science 2013, 339, 328-332.

Servant, G.; Weiner, O. D.; Herzmark, P.; Balla, T.; Sedat, J. W.; Bourne, H. R. Science 2000, 287, $1037-$ 1040.

Ueda, M.; Sako, Y.; Tanaka, T.; Devreotes, P.; Yanagida, T. Science 2001, 294, 864-867.

Van Haastert, P. J. M.; Devreotes, P. N. Nat. Rev. Mol. Cell. Biol. 2004, 5, 626-634.

Kim, M. S.; Khang, G.; Lee, H. B. Prog. Polym. Sci. 2008, 33, 138-164.

Keenan, T. M.; Folch, A. Lab Chip 2008, 8, 34-57.

Genzer, J.; Bhat, R. R. Langmuir 2008, 24, 2294-2317.

Mrksich, M. Acta Biomater. 2009, 5, 832-841.

Simon, C. G.; Lin-Gibson, S. Adv. Mater. 2011, 23, 369-387.

Maier, W. F.; Stöwe, K.; Sieg, S. Angew. Chem. Int. Ed. 2007, 46, 6016-6067.

Potyrailo, R. A.; Mirsky, V. M. Chem. Rev. 2008, 108, 770-813.

Morgenthaler, S.; Zink, C.; Spencer, N. D. Soft Matter 2008, 4, 419-434.

Robertus, J.; Browne, W. R.; Feringa, B. L. Chem. Soc. Rev. 2010, 39, 354-378.

Lin, X.; He, Q.; Li, J. Chem. Soc. Rev. 2012, 41, 3584-3593.

Yu, C.-h.; Groves, J. Med Biol Eng Comput 2010, 48, 955-963.

Malinova, V.; Nallani, M.; Meier, W. P.; Sinner, E. K. FEBS Lett. 2012, 586, 2146.

Schiller, S. M. In Handbook of Biofunctional Surfaces, Chapter 18; Knoll, W., Ed.; Pan Stanford Publishing Pte. Ltd.: Singapore, 2013.

Tamm, L. K.; Mcconnell, H. M. Biophys. J. 1985, 47, 105-113.

Sackmann, E. Science 1996, 271, 43-48.

Salaita, K.; Nair, P. M.; Petit, R. S.; Neve, R. M.; Das, D.; Gray, J. W.; Groves, J. T. Science 2010, 327, 13801385.

Nair, P. M.; Salaita, K.; Petit, R. S.; Groves, J. T. Nat. Protoc. 2011, 6, 523-539.

Perez, T. D.; Nelson, W. J.; Boxer, S. G.; Kam, L. Langmuir 2005, 21, 11963-11968.

Ananthanarayanan, B.; Little, L.; Schaffer, D. V.; Healy, K. E.; Tirrell, M. Biomaterials 2010, 31, 8706-

8715.

Huang, C. J.; Tseng, P. Y.; Chang, Y. C. Biomaterials 2010, 31, 7183-7195.

Charnley, M.; Kroschewski, R.; Textor, M. Integr. Biol. 2012, 4, 1059-1071.

Yu, C. H.; Rafiq, N. B. M.; Krishnasamy, A.; Hartman, K. L.; Jones, G. E.; Bershadsky, A. D.; Sheetz, M. P. Cell Reports 2013, 5, 1456-1468.

Thid, D.; Bally, M.; Holm, K.; Chessari, S.; Tosatti, S.; Textor, M.; Gold, J. Langmuir 2007, 23, 1169311704.

Körner, A.; Deichmann, C.; Rossetti, F. F.; Köhler, A.; Konovalov, O. V.; Wedlich, D.; Tanaka, M. PLoS ONE 2013, 8, e54749.

Groves, J. T.; Boxer, S. G. Biophys. J. 1995, 69, 1972-1975.

Groves, J. T.; Boxer, S. G.; McConnel, H. M. Proc. Natl. Acad. Sci. USA 1997, 94, 13390-13395.

Han, X.; Cheetham, M. R.; Sheikh, K.; Olmsted, P. D.; Bushby, R. J.; Evans, S. D. Integr. Biol. 2009, 1, $205-$ 211.

Lee, Y. K.; Nam, J. M. Small 2012, 8, 832-837.

van Weerd, J.; Krabbenborg, S. O.; Eijkel, J.; Karperien, M.; Huskens, J.; Jonkheijm, P. J. Am. Chem. Soc. 2014, 136, 100-103.

Pace, H. P.; Sherrod, S. D.; Monson, C. F.; Russell, D. H.; Cremer, P. S. Anal. Chem. 2013, 85, 6047-6052. Cheetham, M. R.; Bramble, J. P.; McMillan, D. G. G.; Krzeminski, L.; Han, X.; Johnson, B. R. G.; Bushby, R. J.; Olmsted, P. D.; Jeuken, L. J. C.; Marritt, S. J.; Butt, J. N.; Evans, S. D. J. Am. Chem. Soc. 2011, 133, 65216524.

van Meer, G.; Voelker, D. R.; Feigenson, G. W. Nat. Rev. Mol. Cell. Biol. 2008, 9, 112-124.

Goding, J. W. J. Immunol. Methods 1976, 13, 215-226. 
40 Antibodies: A Laboratory Manual; Harlow, E.; Lane, D., Eds.; Cold Spring Harbor, NY, 1988.

41 Lata, S.; Piehler, J. Nat. Protoc. 2006, 1, 2104-2109.

42 Cabanas Danés, J. PhD, University of Twente, 2013.

43 Sarkar, S.; Isenberg, B. C.; Hodis, E.; Leach, J. B.; Desai, T. A.; Wong, J. Y. Journal of Biomaterials Science, Polymer Edition 2008, 19, 1347-1362.

44 Zander, N.; Orlicki, J.; Rawlett, A.; Beebe, T. Biointerphases 2010, 5, 149-158.

45 Vashist, S. K. Anal. Biochem. 2012, 423, 23-25.

46 Wasserberg, D.; Nicosia, C.; Tromp, E. E.; Subramaniam, V.; Huskens, J.; Jonkheijm, P. J. Am. Chem. Soc. 2013, 135, 3104-3111. 


\section{Chapter 4}

\section{Probing the threshold in bacteria-mannose recognition using SLB gradients*}

Cell-cell recognition events stimulated investigations at the molecular level. For example, the binding of lectins found on pathogenic bacteria to cell-surface carbohydrates. The adhesion of such pathogens to cell-surface carbohydrates is not only dictated by the surface density of these groups but also the magnitude of shear stress from fluid flow. In vivo, this enables pathogens to adhere to cells via their lectins in, for example, the blood stream. This is the case for E. coli binding to mannose via its FimH receptor. To study FimHmannose interaction, we prepared locked-in supported lipid bilayer (SLB) gradients of mannose for the study of FimH presenting ORN 178 bacteria. On the continuous mannose surface gradients specific and density-dependent interaction of ORN 178 bacteria was observed. Moreover, our proposed gradient system allowed for the study of shear stress on the binding behaviour simultaneously. In this case, ORN 178 rolling behaviour as a function of mannose density and shear stress revealed three bacterial population with respect to total distance travelled. The results demonstrate that our chip-based method is useful as a bio-analytical tool to provide insights into early stages of infection.

\footnotetext{
*Manuscript in preparation: J. van Weerd, S. Sankaran, O. Roling, S. Sukas, B.J. Ravoo, M. Karperien and P. Jonkheijm
} 


\subsection{Introduction}

Important cellular processes such as growth, motility, morphology, and differentiation are controlled, in part, by extracellular signals received at the cell's surface. While some external stimuli, such as e.g. hormones and growth factors, are received in soluble form from extracellular fluids, other signals are part of a neighbouring cell surface and exert their effects only through direct cell-cell contact. ${ }^{1}$ Specific cell-surface receptors can interact with a molecular signal on an opposing cell via specific binding to a ligand, and biochemical mechanisms exist to translate that binding into a cellular response. ${ }^{2,3}$ Defects in cell-cell recognition underlie a number of diseases related to uncontrolled cell growth and motility such as in the case of tumour metastasis and embryonic development disorders. ${ }^{4-6}$ Increased understanding of the molecular basis of the cell-cell recognition has made significant implications for improved intervention in many areas of biology and medicine. For example, improved understanding of cell-cell interaction revealed cell sorting processes prior to morphogenesis. ${ }^{4}$ Apart from being involved in tissue development and homeostasis, cell-cell interactions are used by pathogens to invade and infect tissue. Cell-surface carbohydrates that have specific interactions with lectins on pathogens mediate their adhesion and often constitute the initial stage of infection. To forge a tight mutual binding event the surface density of these carbohydrates and shear stress from fluid flow are of utmost importance. For example, pathogenic $E$. coli binding via common adhesive fimbrial lectin protein FimH to mannose carbohydrates is increased upon higher imposed shear stress and higher surface density of mannose. As a result, FimH-mannose interactions allows the bacteria to mitigate the stresses from bodily fluid flow and thus cause infection. 7,8

Glycan arrays have been extremely successful in determining the specificity and affinity of pathogenic lectins towards carbohydrates, however the effect of glycan surface density has been a more difficult parameter to analyse using glycan arrays. ${ }^{9-11}$ Usual strategies require the preparation of several arrays or surfaces with discrete ligand densities, a cumbersome process prone to errors while the information is biased by the pre-selected ligand concentrations. ${ }^{9-11}$ Surface based continuous gradient systems integrated with microfluidics provide insight in the threshold value of the ligand surface density for pathogen recognition under physiologically relevant conditions. ${ }^{12}$ We have adopted supported lipid bilayers (SLB) to prepare our ligand gradient as SLBs have advantageous properties for cellular studies. They have a highly non-fouling nature and are amenable to further chemical functionalization. ${ }^{13-18}$ Previously, we reported a method to conduct $\mu$ SLB electrophoresis to concentrate charged membrane components in a gradient format at low potentials. We were able to impart temporal stability to the gradients using zwitterionic gel-state SLBs that display negligible mobility at room temperature $\left(<10^{-3}\right.$ 
$\mu \mathrm{m}^{2} / \mathrm{s}$ ). This chip-based system also allows us to vary the flow speed and thus the shear stress that is being exerted on components inserted into SLBs. This was previously shown by shear induced flow of streptavidin that was bound to the SLB. Here we describe the fabrication of locked-in SLB-gradients of mannose in a microfluidic system to analyse mannose-FimH interaction that occurs on the mammalian cell surface and pathogenic $E$. coli strains.

\subsection{Results and Discussion}
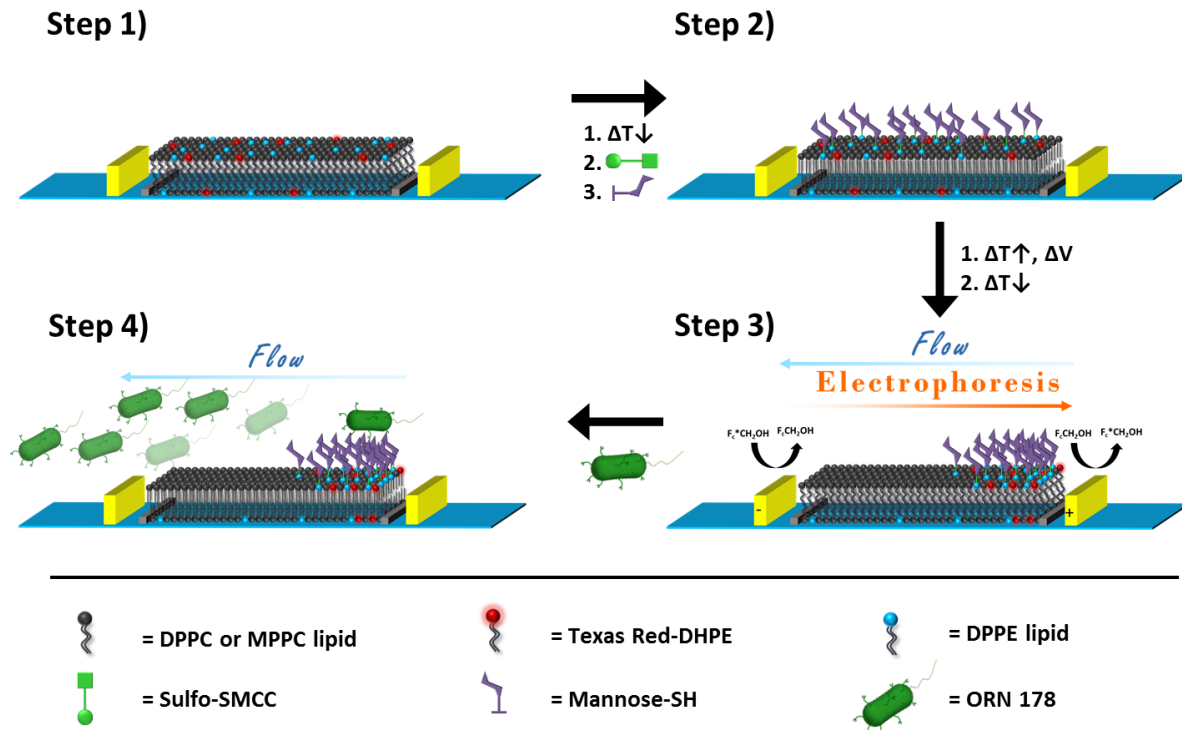

Figure 4.1: Schematic representation of the steps to fabricate gradients of mannose in a SLB to study interactions with bacterial lectins (see text for details).

To prepare continuous gradients of mannose in SLBs (Figure 4.1) we employed $\mu$ SLB electrophoresis chips that have interdigitated gold electrodes (spaced $500 \mu \mathrm{m}$ ) and $\mathrm{Cr}$ corrals of varying length to enable parallel analysis. The chips were first modified with a gel-state SLB based on either DPPC $\left(T_{m} 41^{\circ} \mathrm{C}\right.$, Figure $\left.4.2 \mathrm{a}-i\right)$ or MPPC $\left(T_{m} 35^{\circ} \mathrm{C}\right.$, Figure $4.2 \mathrm{a}-$ ii) by means of vesicle fusion at $60^{\circ} \mathrm{C}$ or $45^{\circ} \mathrm{C}$, respectively, (Figure 4.1 , Step 1 ). The high $\mathrm{T}_{\mathrm{m}}$ of DPPC particularly provides a convenient temperature window in which no lateral mobility is observed. Prior to SLB formation DPPE lipids were included in the vesicles 
a)<smiles>CCCCCCCCCCCCCCCCCCCCCCC(=O)OC[C@H](COP(=O)(O)OCC[N+](C)(C)C)OC(=O)CCCCCCCCCCC</smiles><smiles>CCCCCCCCCCCCCCCCCCCCC(=O)OCC(O)COP(=O)(O)OCC[N+](C)(C)C</smiles>

b)<smiles>CCCCCCCCCCCCCCCCCCCCCCCC(=O)OC[C@@H](OC(=O)CCCCCCCCC)[C@H](C)COP(=O)(O)OCC[NH3+]</smiles>

c)<smiles>O=C(ON1C(=O)CC(S(=O)(=O)[O-])C1=O)C1CCC(CN2C(=O)C=CC2=O)CC1</smiles>

d)<smiles>O=C(CCCOC1O[C@H](CO)[C@@H](O)[C@H](O)[C@H]1O)NCCCS</smiles>

Figure 4.2: Chemical structures; a) Gel-state lipids $i \mathrm{DPPC}, \mathrm{T}_{\mathrm{m}} 41^{\circ} \mathrm{C}$, and ii MPPC, $\mathrm{T}_{\mathrm{m}} 35^{\circ} \mathrm{C}$ b) DPPE lipid, c) Sulfo-SMCC and d) mannose-SH.

(Figure 4.2b) to allow for subsequent modification with a suitable mannose derivative. Fluorescent and negatively charged TR-DHPE lipids were included to allow for visualization of the gradients. After cooling down the chip to room temperature $\left(T<T_{m}\right)$ the DPPE-lipid in the SLB was reacted with heterobifunctional crosslinker sulfo-SMCC (Figure 4.2c). Subsequently, a thiol-modified mannose (Figure 4.2d) was connected to the maleimide group of the sulfo-SMCC linker in a Michael addition reaction to yield mannosefunctionalized SLBs (Figure 4.1, Step 2). ${ }^{19}$ Upon heating the device to $60^{\circ} \mathrm{C}$, in the case of DPPC, and performing $\mu \mathrm{SLB}$ electrophoresis in the presence of $0.5 \mathrm{mM} \mathrm{F}_{\mathrm{c}} \mathrm{CH}_{2} \mathrm{OH}$ at $1 \mathrm{~V}$ potential difference, the mannose-modified DPPE and TR-DHPE lipids displayed electrophoretic migration and build-up against the $\mathrm{Cr}$-corrals due to the net negative charge of -1 on these lipids (see Figure S1 for corral design). After 30 minutes of electrophoretic migration, while flowing the liquid in opposite direction, the chip was 
rapidly cooled down to room temperature to lock the gradients (Figure 4.1, Step 3). To evaluate binding characteristics of bacteria to our mannose gradients, we adhered $E$. coli strain ORN 178, which expresses wild-type FimHK514, and control strain ORN 208, which expresses mutated inactive forms of FimHK514 (Figure 4.1, Step 4). The binding behaviour of the ORN strains to the mannose gradients was monitored at varying flow rates (Figure 4.1 step 4).

The steps of generating the mannose-modified SLB were evaluated using quartz-crystal microbalance with dissipation measurements (QCM-D). To this end, a borosilicate-coated QCM resonator was modified with a MPPC-based SLB, which allowed us to obtain a stable QCM-D signal. The MPPC-SLB was also doped with 0.2 mol\% TR-DHPE and 1 mol\% DPPE. The characteristics of the vesicle fusion process was observed by a change in the frequency and this change levelled around the $-25 \mathrm{~Hz}$, in agreement with observations made in literature (Figure 4.3a). ${ }^{20}$ Both the attachment of the sulfo-SMCC linker as well as the subsequent conjugation of mannose-SH could be followed by monitoring changes in frequency and dissipation. These changes stabilized after 30 and 50 minutes, respectively (Figure $4.3 \mathrm{~b}-\mathrm{c}$ ) indicating that the reaction was complete. A larger shift in $\Delta D$ and $\Delta f$ was observed in the case of attaching the mannose derivative, presumably related to a more flexible, polar and extended structure involving additional coupled water. ${ }^{21}$ The presence and binding properties of the mannose-modified SLBs was evaluated by following the change in frequency and dissipation upon flowing a $1 \mu \mathrm{M}$ solution of a lectin protein Conconavalin A (ConA) over the chip. ConA is homotetrameric lectin that is able to bind mannose. ${ }^{22}$ Binding of ConA to the mannose-modified SLB occurred within 15 minutes and only slowly dissociated when rinsed with buffer (Figure 4.3d). Upon introduction of a 10 $\mathrm{mM}$ mannose solution, fast dissociation of ConA from the SLB was observed. No ConA binding was observed to unmodified MPPC-SLB doped with DPPE and TR-DHPE further verifying that the interaction of ConA and mannose was specific (Figure S2). It furthermore demonstrated absence of protein binding to the SLB in line with its non-fouling properties. The QCM-D experiments confirmed that the SLBs can be modified with mannose moieties that were able to specifically bind with proteins.

We next fabricated mannose-modified SLB gradients as described in Figure 4.1 on the $\mu S L B$ electrophoresis chips. Upon separate incubations of the mannose-modified SLBS with strain ORN 178 and the control strain ORN 208, we observed selective binding after flushing the surface at $100 \mu \mathrm{L} \mathrm{min}{ }^{-1}$. In Figure 4.4a bright field and fluorescent microscopy images are shown of bacteria adhered to a mannose gradient prepared with $0.4 \mathrm{~mol} \%$ DPPE-mannose. TR-DHPE serves as a suitable marker for the electrophoretic build-up of 
DPPE-mannose due a similar lipid structure, charge and molecular weight. ${ }^{23}$ The TR-DHPE fluorescence image in Figure 4.4a exhibited a distinct fluorescence build-up along a 430

a)

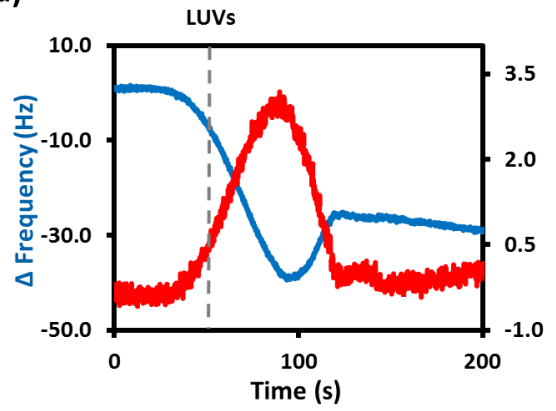

c)

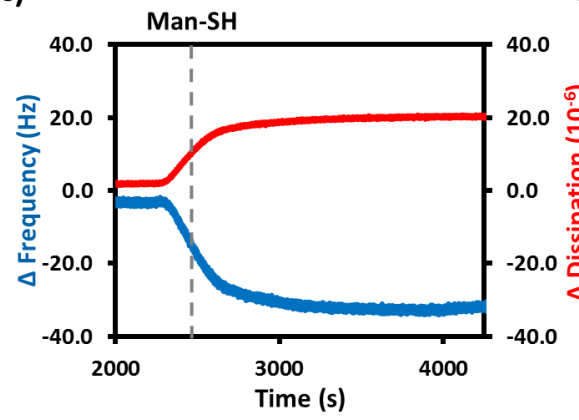

b)

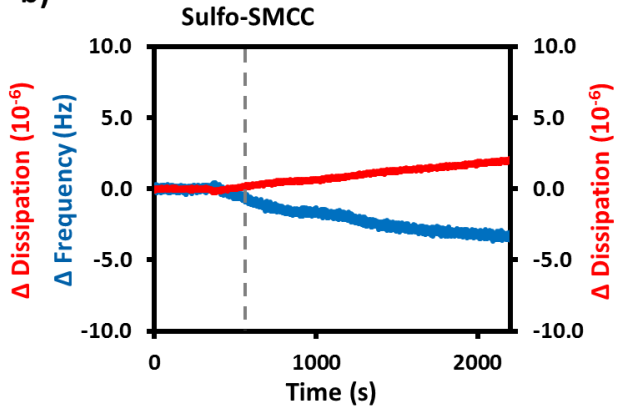

d)

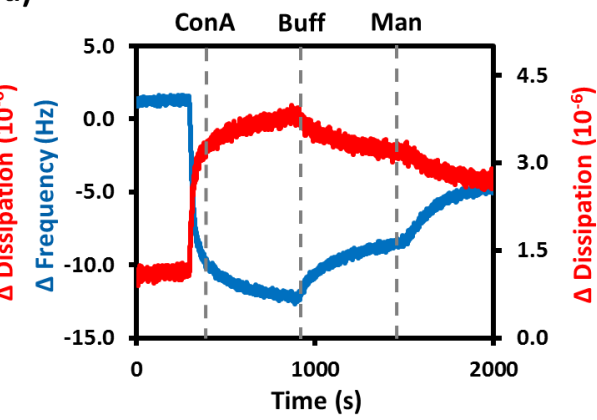

Figure 4.3: Evaluating mannose-modified SLBs with QCM-D; a) SLB formation of a MPPC-based SLB doped with $1 \mathrm{~mol} \%$ DPPE and $0.2 \mathrm{~mol} \%$ TR-DHPE at $45^{\circ} \mathrm{C}$ on a borosilicate resonator (dashed line indicates addition of LUVs). b) Subsequent reaction of $5 \mathrm{mM}$ sulfo-SMCC at r.t. with DPPE for 30 minutes at pH 7.4 (dashed line indicates addition of sulfo-SMCC). c) Reaction with $1 \mathrm{mM}$ mannose$\mathrm{SH}$ for 30 minutes at pH 6.8 (dashed line indicates addition mannose-SH (Man-SH). d) Binding of 1 $\mu \mathrm{M}$ ConA to the mannose-modified SLB. Dashed lines from left to right represent; addition of ConA, buffer wash (Buff) and addition of $10 \mathrm{mM}$ mannose (Man). Data presented for $\Delta D_{n=5}$ and $\Delta f_{n=5}$.

$\mu \mathrm{m}$ long corral. In the region of the upper limit of the gradient as determined from the TRDHPE fluorescence, an increased amount of adhered ORN 178 can be clearly noted after applying flow. The ORN 178 cell count increased with increasing surface density of mannose (Figure 4.4b) whereas only negligible binding of control ORN 208 (Figure 4.4c) occurred to the gradient i.e. ca. 5\% compared to ORN 178 after applying flow. Control experiments performed on unmodified DPPC-based SLBs doped with DPPE and TR-DHPE revealed no interaction with the zwitterionic SLB, confirming specific interaction of the 
FimH receptor with DPPE-mannose. To widen the probing window of our gradients, different mannose-modified SLB gradients were prepared by varying the doping densities of DPPE (0.1, 2 and 4 mol\%) prior to $\mu$ SLB electrophoresis. For each mannose-modified SLB gradient, the corrals were divided into five bins $(i-v)$ and the average interligand spacing for each bin was calculated based on the TR-DHPE build-up. Based on the binding of ORN 178 to these mannose-modified SLB gradients, the bacterial immobilization is plotted as a function of mannose carbohydrates per area unit (Figure 4.4d).

a)

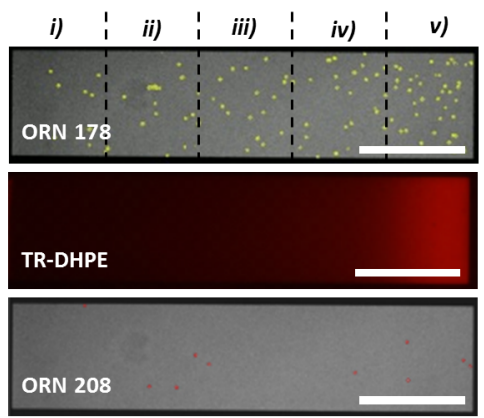

c)

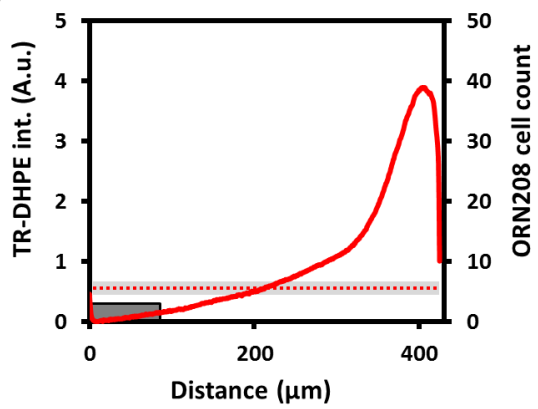

b)

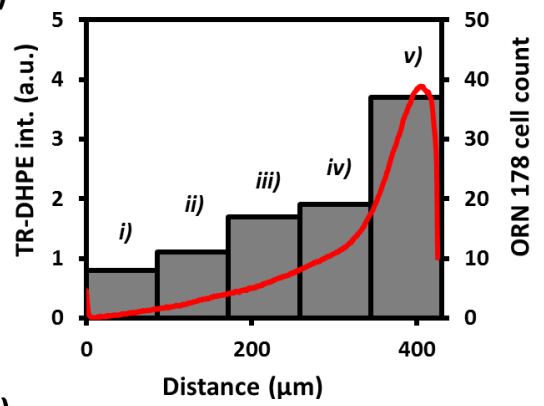

d)

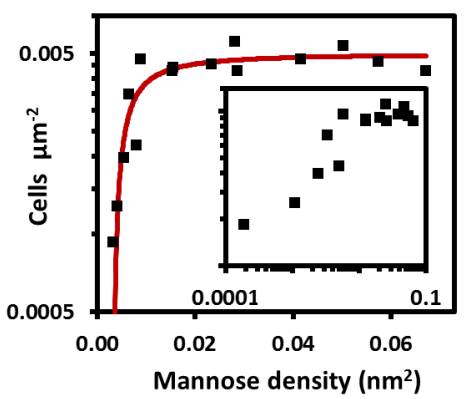

Figure 4.4: Bacterial surface gradient on 0.4 mol\% DPPE-mannose; a) Bright field micrographs of ORN 178 (marked green for clarity reasons) and ORN 208 (marked red for clarity reasons) binding at 100 dynes $\mathrm{cm}^{-2}$ to the DPPE-mannose gradient indicated by TR-DHPE fluorescence, scale bar $100 \mu \mathrm{m}$. Quantification of cell count vs TR-DHPE fluorescence of b) ORN 178 and c) ORN 208. In c) the dotted red line indicates the mean start amount of ORN 208, grey region represents the SD d) Number of bound ORN 178 cells per $\mu \mathrm{m}^{2}$ as a function of mannose surface density (Black squares). The solid red line is to guide the eye. Inset shown log-log plot

This plot demonstrates that a significant rise in the number of adhered bacterial cells occurred by an order of magnitude only at a very narrow window of rising mannose densities from 0.0020 to $0.0040 \mathrm{~nm}^{2}$. Beyond 0.0050 mannose moieties per $\mathrm{nm}^{2}$, the 
number of adhered bacterial cells remains constant. These results confirm that the availability of ligand-modified SLB gradients on a single chip is powerful to evaluate the selective binding of cells as the density and slope of the ligand presentation in the gradient details the threshold for pathogen recognition to the ligands.

a)

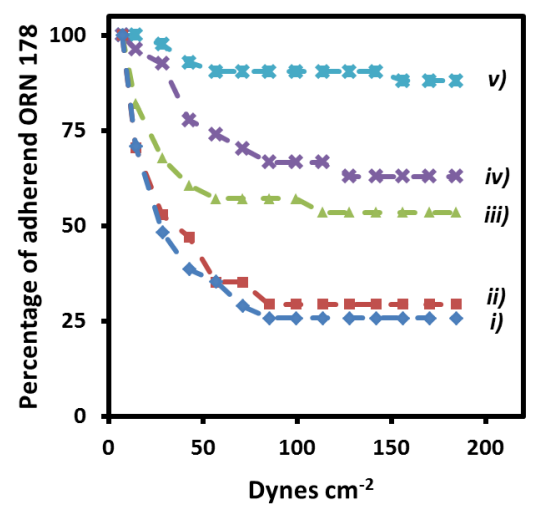

b)

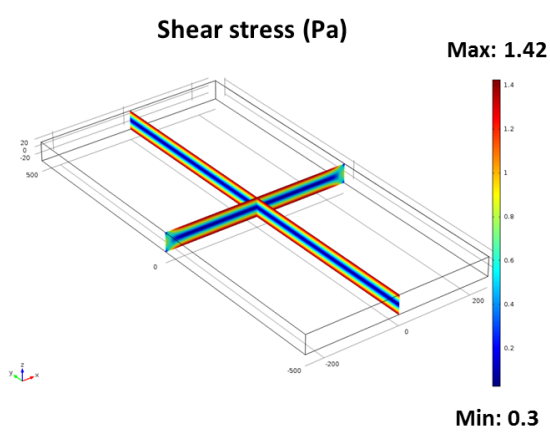

c)

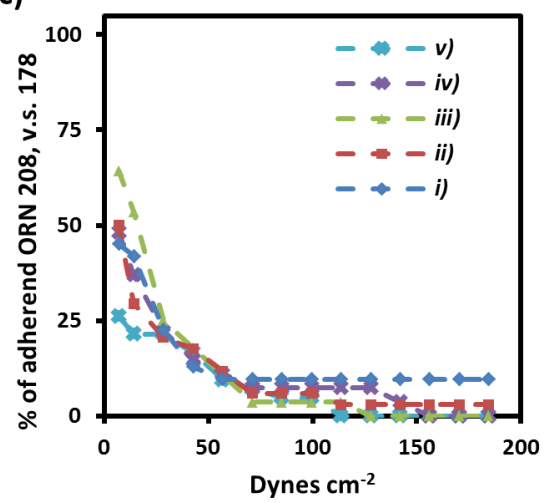

Figure 4.5: Influence of shear stress on ORN 178 and ORN 208 binding to locked-in mannose gradients; Effect of increasing shear stress on ORN 178 bound to mannose-gradients. a) Percentage of adherent ORN 178 vs. shear stress, three regimes were found. b) Longitudinal and axial shear stress distributions at $1,000 \mu \mathrm{L} \mathrm{hr}^{-1}$. c) Percentage of adhered ORN 208, normalized against ORN 178 data, subjected to varying shear stresses.

To further exploit the mannose-modified SLB gradients on our chip, we next varied the flow speed within the channel to investigate the effect of shear stress on the adhered bacteria (Figure 4.5a). To accurately describe the shear stress near the surface, a finite elements simulation was performed (Figure 4.5b) showing that shear stress values of up to 
ca. 200 dynes $\mathrm{cm}^{-2}$ could be evaluated in our device. In vivo, wall shear stress in human blood vessels range from tenth, to several hundreds of dynes $\mathrm{cm}^{-2}$ for e.g. veins and stenotic vessels, respectively. ${ }^{24}$ In the region of high bacterial cell density driven by the high surface density of mannose, the majority (88\%) of ORN 178 cells were able to remain bound under increasing shear stress. Whereas in the region with the highest density of mannose (bin $v$ ) only a small fraction of bacterial cells were lost from the gradient at high shear stress, in the two regions with the lowest density of mannose (bins $i$ and ii) a significant reduction (75\%) of bound bacterial cells was observed, while bins iii and iv represented an intermediate case (40\%). In the absence of flow and in the presence of cells, specific interaction of ORN 178 with the mannose surface resulted in a two times higher initial cell density compared to ORN 208 (Figure 4.5c). When these ORN 208 cells were subjected to flow and at increasing shear stress, ca. $95 \%$ of the initially adhered bacteria were washed away indicating that these ORN 208 were non-specifically adhered (Figure 4.5c). Our findings support that the typical catch-bond formation between mannose and FimH receptor had occurred. This is evidenced by the bacterial adhesion in e.g. bins iii and iv where we note a decrease in cell detachment upon increase in shear stress. Similar effects have been observed on surfaces coated with monomannosylated BSA (mono-mannose). In this case at ca. 50 dynes $\mathrm{cm}^{-2}$ the fraction of firmly attached cells levelled off, similar to the results in Figure $4.5 \mathrm{a} .{ }^{25}$ On the contrary, the region with the highest density of mannose (bin $v$ ) showed very little loss of bacteria upon increase in shear stress, reminiscent of bacterial adhesion on RNaseB coated surfaces (tri-mannose) surfaces under increasing shear stress. ${ }^{25,26}$ Illustrating that the mannose-SLB gradient enables the simultaneous analysis of mannose density and shear stress on bacterial binding.

In all cases, the surface bound bacterial cells moved across the surface in the flow direction each time promptly after the flow speed was increased. This bacterial cell immediate movement could not be observed in time in the case the flow speed was kept constant. This is in agreement with literature data where E. coli movement in time was exclusively observed at very low shear stress, i.e. $<10$ dynes $\mathrm{cm}^{-2}$, which is below the lowest shear stress tested in this study i.e. 14.2 dynes $\mathrm{cm}^{-2} .8$ Beyond the shear stress range that was tested in that study, we observed an immediate response to changes in shear stress. The bacterial response is presumably related to stick-and-roll rolling motion of cells that is physiologically observed. ${ }^{7,8,27}$ When considering three regions of mannose density in the SLB gradient (bins $i$ and $i$, bins iii and iv and bin $v$ ), three distinct bacterial populations can be discerned with respect to the total bacterial displacement as a result of rolling during the course of the experiment (Figure 4.6a). During the shear stress experiments on all parts of the mannose-modified SLB gradient, ORN 178 cells were identified that traversed across the SLB with total distances that can be grouped in 0-5, 5- 
10 and $10-15 \mu \mathrm{m}$. The absolute amount of cells per $\mu \mathrm{m}^{2}$ that moved less than $5 \mu \mathrm{m}$ was significantly highest for the region of the highest density of mannose (bin $v$ ) indicating that stronger surface interactions were experienced by the bacteria in this region (Figure 4.6b). Moreover, the fraction of the cells within each bin that moved a distance of $10-15 \mu \mathrm{m}$ decreased with increasing mannose surface density (Figure 4.6c), supporting stronger surface interaction in those regions. Although this stick-and-roll rolling motion has been observed in vitro at low shear stress levels on fully coated mono- and tri-mannose samples, ${ }^{27}$ to our knowledge this behaviour has not been studied on more dilute mannose surfaces in a broader shear stress regime. Recently, it has been shown that $E$. coli displacement could be caused by FimH-mannose bonds that form and break according to the two-state, allosteric catch-bond model, or due to shear-stabilized rolling. The latter is thought to be caused by an increase in the low-affinity bonds due to increased fimbrial deformation with shear. ${ }^{28}$ Our observations might indicate different adhesion and rolling processes that have occurred and their propensity in relation to mannose density and shear stress.

a)

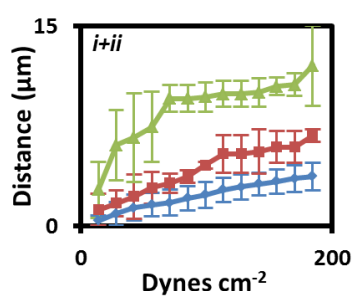

b)

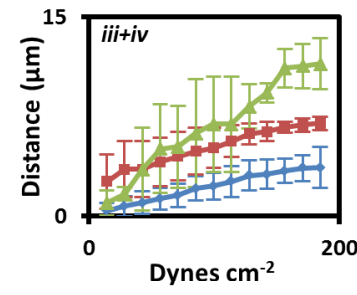

c)
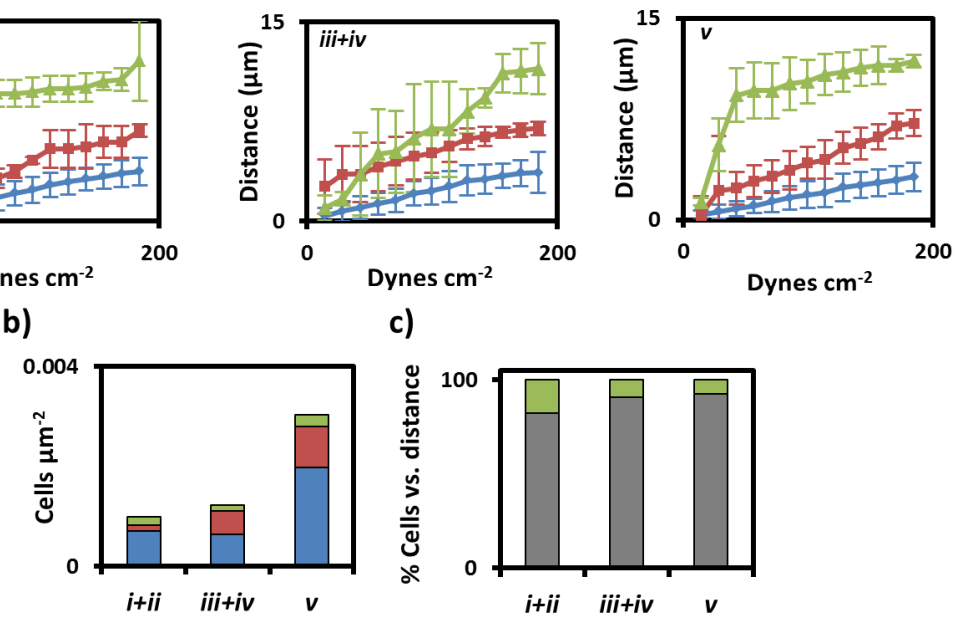

Figure 4.6: Bacterial displacement vs. shear stress; ORN 178 displacement vs. in shear stress per regime of mannose density i.e. bins $i+i i, i i i+i v$ and $v$. Data is presented for cells that remained bound throughout the experiment. Three populations within each regime were identified with respect to total distance travelled. a) Distance travelled vs, shear stress, data presented as mean \pm SD. The absolute amounts of the three populations per regime is given in $\mathbf{b})$. The fraction of bacterial cells that travelled a distance of $10-15 \mu \mathrm{m}$ per regime is presented in c). The three populations of bacterial displacement are presented as 0-5 (Blue), 5-10 (Red) and 10-15 (Green) $\mu \mathrm{m}$ travelled. 


\subsection{Conclusions}

Locked-in gradients of mannose were prepared, characterized and used to evaluate the binding of $E$. coli strain ORN 178. FimH lectin binding to the continuous locked-in mannose gradients is specific and shows selective binding above a threshold density of mannose. The non-fouling properties of the SLB surface ensured absence of non-specific binding of bacteria. The chips were applied to study the binding of ORN 178 cells under application of shear stress. Bacteria bound to highest regions of mannose on the SLB gradient are most prone to retain their cell-surface interactions. Bacterial displacement was noted upon a change in shear stress which is indicative of rolling behaviour and was found to be inversely related to mannose density. Our SLB gradient chip proves instrumental for probing the threshold in pathogen-lectin interactions in a semi-high-throughput fashion by easily varying the ligand density over a wide range on one chip under physiologically relevant shear conditions on a non-fouling SLB surface.

\subsection{Acknowledgements}

Dr. S. O. Krabbenborg is thankfully acknowledged for fabricating the $\mu$ SLB electrophoresis chips.

\subsection{Experimental section}

\subsubsection{Materials}

All starting materials and chemicals were purchased from Sigma-Aldrich, Fluka, Serva, Becton Dickinson, Avanti Polar Lipids, Microchem, Thermo Fisher and Invitrogen, and they were used as received, unless otherwise stated. Mannose-SH was kindly provided by $\mathrm{O}$. Roling and B.J. Ravoo and synthesized according to a literature procedure. ${ }^{29}$ Both ORN 178 and ORN 208 bacterial strain were kindly provided by Prof. Luc Brunsveld, TU/e. MilliQ water with a resistivity higher than $18 \mathrm{M} \Omega \mathrm{cm}^{-1}$ was used in all experiments.

\subsubsection{Methods}

Chip fabrication, PDMS flow channel and PDMS bonding. For more information consult experimental section of Chapter 2. Additional care was taken to use a fresh $\mathrm{F}_{\mathrm{c}} \mathrm{CH}_{2} \mathrm{OH}$ solution for gradient formation of the DOPS lipids to ensure a near $\mathrm{pH}$-neutral solution.

Vesicle preparation. 1,2-Dipalmitoyl-sn-glycero-3-phosphocholine (DPPC, Avanti Polar Lipids) was stored as a $10 \mathrm{mg} / \mathrm{mL}$ stock solution in chloroform at $-20^{\circ} \mathrm{C}$. $1,2-$ 
Dihexadecanoyl-sn-glycero-3-phosphoethanol-amine (DPPE, Avanti Polar Lipids) was stored as a $4 \mathrm{mg} / \mathrm{mL}$ stock in chloroform at $-20^{\circ} \mathrm{C}$. The charged lipid-dye conjugate, Texas Red-1,2-dihexadecanoyl-sn-glycero-3-phosphoethanolamine (TR-DHPE, Invitrogen) was stored as a $1 \mathrm{mg} / \mathrm{mL}$ stock solution in methanol at $-20^{\circ} \mathrm{C}$. Desired molar ratios of the DPPC, DPPE and TR-DHPE were mixed and dried under a flow of nitrogen in a glass vial, and subsequently placed for at least 1 hour. The resulting lipid film was re-suspended by vortexing in MilliQ water to form multilamellar vesicles (MLVs) at $1 \mathrm{mg} / \mathrm{mL}$ at $60^{\circ} \mathrm{C}$. The MLV solution was extruded 11 times through a $100 \mathrm{~nm}$ polycarbonate membrane (Avanti Polar Lipids) at $60^{\circ} \mathrm{C}$. The resulting large unilaminar vesicles (LUVs) were kept at room temperature and used within two weeks.

SLB formation. SLB formation was performed as described in Chapter 3, with one modification, i.e. after vesicle fusion, rinsing with MilliQ water ( $300 \mu \mathrm{L} / \mathrm{min})$ and cooling down, the chip was flushed with $50 \mathrm{mM}$ sodium phosphate buffer (Sigma-Aldrich, pH 7.4).

Mannose coupling. To conjugate mannose to the primary amine of DPPE $5 \mathrm{mM}$ of sulfosuccinimidyl 4-(N-maleimidomethyl)cyclohexane-1-carboxylate (Sulfo-SMCC) in 50 $\mathrm{mM}$ sodium phosphate buffer $(\mathrm{pH}$ 7.4) was allowed to react for 1 hour at room temperature. Subsequently, $1 \mathrm{mM}$ mannose-SH in $50 \mathrm{mM}$ sodium phosphate (SigmaAldrich pH 6.8) was flown through the device and left to react for 1 hour. Care was taken to prepare fresh solutions in order to prevent NHS hydrolysis and disulfide formation, respectively. Afterwards the chip was extensively rinsed with milliQ prior to $\mu \mathrm{SLB}$ electrophoresis.

$\mu S L B$ electrophoresis. Electric fields were generated and currents were measured with a $\mathrm{CH}$ Instruments 760D potentiostat with the counter and reference connections shorted. During $\mu \mathrm{SLB}$ electrophoresis, a freshly prepared solution of $0.5 \mathrm{mM}$ of $\mathrm{F}_{\mathrm{c}} \mathrm{CH}_{2} \mathrm{OH}$ (SigmaAldrich) in MilliQ water was flushed through the chip at $100 \mu \mathrm{L} / \mathrm{min}$. Prior to heating the device a potential difference of $1 \mathrm{~V}$ was applied for at least 30 seconds at room temperature. After that the chip was heated to $60^{\circ} \mathrm{C}$ and the potential difference of $1 \mathrm{~V}$ was applied for 30 minutes. Subsequently, the chip was cooled down rapidly on a heat exchanger for 5 minutes to fix the mannose surface gradient.

Bacterial cell culture. The bacterial strains ORN 178 and ORN 208 were grown overnight in LB media (Sigma-Aldrich) using tetracycline (Sigma-Aldrich) as the selective antibiotic. These were then spun down at 5,000 $\times g$ for 10 minutes and the supernatant was discarded. The bacteria were washed twice with $10 \mathrm{mM}$ HEPES, $137 \mathrm{mM} \mathrm{NaCl}$, pH 7 buffer 
by centrifugation and resuspension. Finally the bacteria were reconstituted in this buffer and their optical density (OD) at $600 \mathrm{~nm}$ was measured.

Bacterial adhesion. OD 0.1 solutions of ORN 178 and ORN 208 were prepared in the above mentioned HEPES $\mathrm{NaCl}$ buffer. Prior to flowing the bacteria through the device, $\mathrm{MnCl}_{2}$ and $\mathrm{CaCl}_{2}$ were added at a final concentration of $1 \mathrm{mM}$. $200 \mu \mathrm{L}$ of bacterial solution was flown through the chip at $100 \mu \mathrm{L} / \mathrm{min}$. The flow was stopped and the cells were left to settle for 15 minutes. After the set incubation period, the flow speed was increased incrementally to study the effect of shear stress as well as surface mannose density.

Fluorescence microscopy. An Olympus inverted IX71 epi-fluorescence research microscope with a Xenon X-cite 120PC as light source and a digital Olympus DR70 camera for image acquisition was used to acquire fluorescence micrographs of the TR-DHPE gradient. TR-DHPE was imaged using $510 \leq \lambda_{\mathrm{ex}} \leq 550 \mathrm{~nm}$ and $\lambda_{\mathrm{em}}>590 \mathrm{~nm}$. Bacterial adhesion was imaged using top bright field illumination. In both instances ISO200 camera settings were used to record high quality, low noise images. In the case of fluorescence micrographs, care was taken to ensure image acquisition was performed in the linear response regime.

QCM-D. SLBs were formed on borosilicate QCM resonators (Q-Sense, QSX 336). Prior to use, the resonators were cleaned with Piranha for 5 minutes and rinsed with milliQ. After mounting the cleaned resonators in the QCM-D holder. The temperature was set at $45^{\circ} \mathrm{C}$ allowed to equilibrate while flowing $0.5 x$ degassed 0.01 M PBS (Sigma-Aldrich, pH 7.4). After obtaining a stable baseline (less than $\Delta 0.5 \mathrm{~Hz}$ during 10 minutes), $0.5 \mathrm{mg} / \mathrm{mL}$ LUVs were flown through the device. The $1 \mathrm{mg} / \mathrm{mL}$ stock LUV solution in milliQ was diluted 1:1 with 0.01 M PBS (Sigma-Aldrich, pH 7.4) just before addition of vesicles. After monitoring the vesicle fusion process, the temperature was lowered to $22^{\circ} \mathrm{C}$ in the presence of the LUV solution. Subsequently, $50 \mathrm{mM}$ sodium phosphate buffer (Sigma-Aldrich, $\mathrm{pH}$ 7.4) was passed through until a stable baseline was reached. A freshly prepared $5 \mathrm{mM}$ sulfo-SMCC solution in $50 \mathrm{mM}$ sodium phosphate buffer (Sigma, Aldrich, $\mathrm{pH}$ 7.4) was allowed to react for 30 minutes. Subsequently, a $1 \mathrm{mM}$ mannose-SH solution in $50 \mathrm{mM}$ sodium phosphate buffer (Sigma-Aldrich, $\mathrm{pH}$ 6.8) was allowed to react for 30 minutes. A $1 \mu \mathrm{M}$ Concanavalin A (Sigma-Aldrich) solution in $\mathrm{pH} 7 \mathrm{HEPES} \mathrm{NaCl}$ buffer containing $1 \mathrm{mM} \mathrm{MnCl}$ and $\mathrm{CaCl}_{2}$ was flown through the device to monitor binding. All fluids were exchanged continuously at

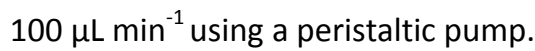


Data analysis. Image analysis was performed using ImageJ (NIH), Origin (OriginLab) and Excel (Microsoft).

Simulation of shear stress. Finite elements analysis (COMSOL Multiphysics) was performed to gain insights into the shear stress at certain flow speeds in our device. A simulation domain of $500 \mu \mathrm{m} \times 50 \mu \mathrm{m} \times 1 \mathrm{~mm}$ was used to model the flow channel. Navier-Stokes equations were solved for incompressible laminar flow (very low Reynolds number) with no-slip boundary conditions at the walls. Constant volumetric flow rate was defined at the inlet as laminar in-flow with $2 \mathrm{~mm}$ entrance length. The 3D model consisted of 430,000 elements to reach mesh independent convergence. For the simulations, water was taken as the model liquid. A calculated wall shear stress per unit flow rate of $1.42 \mathrm{x}$ $10^{-3} \mathrm{~Pa} \mu \mathrm{L}^{-1} \mathrm{hr}^{-1}$ was found. Experiments were performed to prove that no back pressure was evident at the flow speeds used herein.

\subsection{References}

Harris, T. J. C.; Tepass, U. Nat. Rev. Mol. Cell Biol. 2010, 11, 502-514.

Ottone, C.; Krusche, B.; Whitby, A.; Clements, M.; Quadrato, G.; Pitulescu, M. E.; Adams, R. H.; Parrinello, S. Nat. Cell Biol. 2014.

Peglion, F.; Llense, F.; Etienne-Manneville, S. Nat. Cell Biol. 2014, 16, 639-651.

Bischoff, M.; Schnabel, R. Dev. Biol. 2006, 294, 432-444.

Etienne-Manneville, S. Oncogene 2008, 27, 6970-6980.

Zaromytidou, A.-I. Nat. Cell Biol. 2012, 14, 990-990.

Thomas, W. E.; Trintchina, E.; Forero, M.; Vogel, V.; Sokurenko, E. V. Cell 2002, 109, 913-923.

Nilsson, L. M.; Thomas, W. E.; Sokurenko, E. V.; V, V. Appl. Environ. Microbiol. 2006, 72, 3005-3010.

Bouckaert, J.; Mackenzie, J.; de Paz, J. L.; Chipwaza, B.; Choudhury, D.; Zavialov, A.; Mannerstedt, K.; Anderson, J.; Pierard, D.; Wyns, L.; Seeberger, P. H.; Oscarson, S.; De Greve, H.; Knight, S. D. Mol. Microbiol. 2006, 61, 1556-1568.

Liang, P. H.; Wang, S. K.; Wong, C. H. J. Am. Chem. Soc. 2007, 129, 11177-11184.

Pieters, R. J. Org. Biomol. Chem. 2009, 7, 2013-2025.

Krabbenborg, S. O.; van Weerd, J.; Karperien, M.; Jonkheijm, P.; Huskens, J. ChemPhysChem 2014, 15, 3460-3465.

Tamm, L. K.; Mcconnell, H. M. Biophys. J. 1985, 47, 105-113.

Sackmann, E. Science 1996, 271, 43-48.

Salaita, K.; Nair, P. M.; Petit, R. S.; Neve, R. M.; Das, D.; Gray, J. W.; Groves, J. T. Science 2010, 327, $1380-$ 1385.

Nair, P. M.; Salaita, K.; Petit, R. S.; Groves, J. T. Nat. Protoc. 2011, 6, 523-539.

Malinova, V.; Nallani, M.; Meier, W. P.; Sinner, E. K. FEBS Lett. 2012, 586, 2146.

Schiller, S. M. In Handbook of Biofunctional Surfaces, Chapter 18; Knoll, W., Ed.; Pan Stanford Publishing Pte. Ltd.: Singapore, 2013.

Ashley, C. E.; Carnes, E. C.; Phillips, G. K.; Padilla, D.; Durfee, P. N.; Brown, P. A.; Hanna, T. N.; Liu, J.; Phillips, B.; Carter, M. B.; Carroll, N. J.; Jiang, X.; Dunphy, D. R.; Willman, C. L.; Petsev, D. N.; Evans, D. G.; Parikh, A. N.; Chackerian, B.; Wharton, W.; Peabody, D. S.; Brinker, C. J. Nat. Mater. 2011, 10, 389-397. Richter, R. P.; Brisson, A. R. Biophys. J. 2005, 88, 3422-3433.

Hook, F.; Kasemo, B.; Nylander, T.; Fant, C.; Sott, K.; Elwing, H. Anal. Chem. 2001, 73, 5796-5804.

Dechtrirat, D.; Gajovic-Eichelmann, N.; Wojcik, F.; Hartmann, L.; Bier, F. F.; Scheller, F. W. Biosens. Bioelectron. 2014, 58, 1-8. 
Groves, J. T.; Boxer, S. G.; McConnel, H. M. Proc. Natl. Acad. Sci. USA 1997, 94, 13390-13395. Brown, D. L. Cardiovascular plaque rupture; Marcel Dekker: New York, 2002. Nilsson, L. M.; Thomas, W. E.; Trintchina, E.; Vogel, V.; Sokurenko, E. V. J. Biol. Chem. 2006, 281, 1665616663.

Moller, J.; Luhmann, T.; Chabria, M.; Hall, H.; Vogel, V. Sci. Rep. 2013, 3.

Thomas, W. E.; Nilsson, L. M.; Forero, M.; Sokurenko, E. V.; Vogel, V. Mol. Microbiol. 2004, 53, 15451557.

Whitfield, M.; Ghose, T.; Thomas, W. Biophys. J. 2010, 99, 2470-2478.

Wendeln, C.; Rinnen, S.; Schulz, C.; Kaufmann, T.; Arlinghaus, H. F.; Ravoo, B. J. Chem. Eur. J. 2012, 18, 5880-5888. 


\subsection{Supporting information}

a)

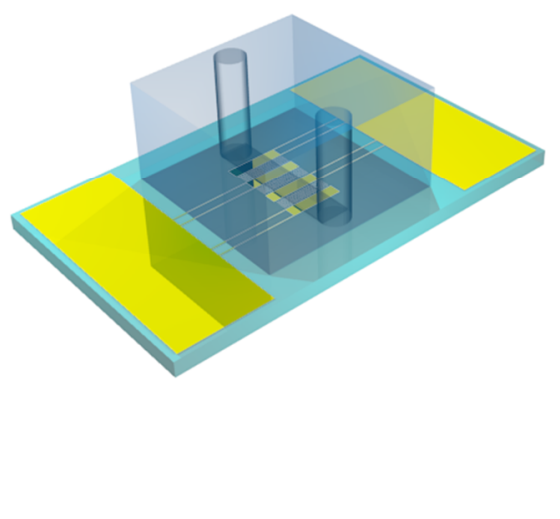

b)

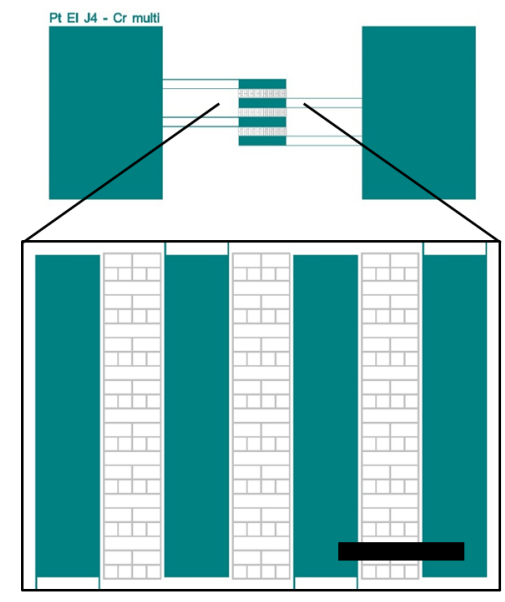

Figure S1: Chip corral design; a) Schematic of the $\mu$ SLB electrophoresis chip with PDMS flow channel bonded to the surface. b) Chip design showing the different coral lengths, scale bar $1 \mathrm{~mm}$.

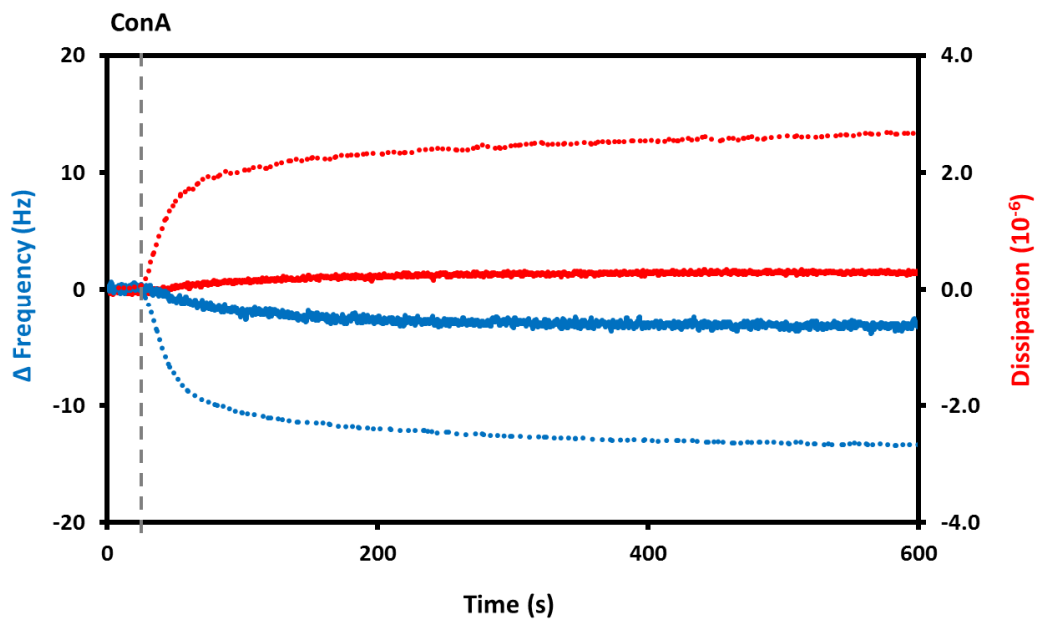

Figure S2: ConA binding on DPPE vs. DPPE-mannose functionalized MPPC-SLB; The solid curve represent the QCM-D response of DPPE and the dotted curve of DPPE-mannose to ConA addition. Data presented for $\Delta D_{n=5}$ and $\Delta f_{n=5}$. 


\section{Chapter 5}

\section{Tuning cell adhesion, spreading and matrix formation of cells in vitro using SLBs ${ }^{*}$}

The cell repellent and non-fouling properties in combination with functionalization strategies of supported lipid bilayers (SLBS) open the opportunity for fundamental studies aimed at understanding the role of cell-extracellular matrix (ECM) interactions in cell behaviour and stem cell differentiation. In the present work, the synthesis of an RGD ligand is described that can be incorporated in SLBs to specifically interact with cells. The density and mobility of the RGD ligand can be varied and is used to steer cell adhesion and spreading. Human primary chondrocytes were used as a model cell system and their morphology was varied from single spheroid cells, single spread cells to the formation of cellular clusters of chondrocytes. In addition, the lateral mobility of the RGD ligands was found to influence the focal adhesion assembly and the cytoskeletal organization of chondrocytes. Moreover, the matrix deposition of a preosteoblastic cell line was modulated by a change in the lateral mobility of the RGD ligands in either liquid or gelstate SLBs. Apart from the well-studied interligand spacing, altering the lateral mobility of ligands in SLBs offers new opportunities in studying and directing (stem) cell fate.

J. van Weerd, N. Georgi, K.E. de Rooij, A. Chan, M. Karperien and P. Jonkheijm (2014) manuscript in preparation 


\subsection{Introduction}

Cartilage can be found on surfaces of articulating joints where it prevents direct bone-tobone contact and reduces friction. Cartilage tissue can be damaged by wear, trauma or diseases like degenerating joint conditions such as osteoarthritis. The low healing capacity of damaged cartilage tissue results in progressive degradation of the tissue eventually leading to impaired joint function. Therefore cell-based cartilage repair strategies have been proposed such as ${ }^{1-4}$ autologous chondrocyte transplantation. In vivo, chondrocytes are found in cartilage tissue where they produce and maintain the cartilaginous matrix. ${ }^{4}$ In autologous chondrocyte transplantation, healthy cartilage tissue is explanted from the patient and chondrocytes are isolated and expanded in vitro. Due to the low cell-to-matrix ratio in cartilage tissue, $2-3 \%$, cell expansion is essential to obtain sufficiently large amounts of chondrocytes to repair the tissue defect. However, in vitro 2D cell expansion often results in chondrocyte dedifferentiation, which is characterized by the loss of chondrogenic phenotype and tissue repair capacity. ${ }^{5}$ Dedifferentiation is observed when chondrocytes are isolated from their native ECM and are cultured under conditions that promote a flattened cell morphology indicated by the formation of actin stress fibres. ${ }^{6}$ Low attachment 3D cell culture platforms like agarose microwells or hydrogels that mimic the native environment, are studied to prevent chondrocyte dedifferentiation. ${ }^{7}$ In this case promising results have been achieved showing that chondrocytes can maintain their spheroid morphology and partially retain their phenotype. ${ }^{7}$ In addition, growth factors like transforming growth factor beta (TGF- $\beta$ ) have been used to maintain a chondrocyte phenotype. ${ }^{8}$ Yet most approaches lack the simultaneous mimicking of cell-ECM-interaction and maintenance of round morphology.

An alternative approach to design cell culture platforms that allow for maintaining the native (spheroid) morphology of chondrocytes would be to explore the possibility to culture human articular chondrocytes on supported lipid bilayers (SLBs). SLBs as in vitro cell culture platforms allow for the study of both cell-cell interaction as well as cell-ECM interaction, as reviewed in Chapter 1. For example, SLBs decorated with RGD-peptides have been used to mimic e.g. the ECM to promote neural cell adhesion and to study podosome formation in fibroblasts. ${ }^{9,10}$ An important consideration in such studies is how to optimize the engagement of the RGD ligands with transmembrane integrins. By variations in the RGD doping density in SLBs the change in interligand spacing of the RGD ligands led to changes in adhesion and spreading of HEK293 cells. HEK293 cells cultured on SLBs doped with 1 mol\% of an RGD-lipid, which relates to an interligand spacing of ca. 10 $\mathrm{nm}$, were able to flatten and form filopodial extensions and show cell migration. In contrast, lower densities of RGD-lipids caused HEK293 cells to adopt a spheroid morphology. ${ }^{11}$ Another possible consideration when using SLBs for cell culture is the 
fluidity of the membrane and components tethered to the membrane. Changing from a liquid-state to a gel-state SLB has been shown to prevent clustering of epidermal growth factors in breast cancer cell line, MCF-7. ${ }^{12}$ Other work showed an increased adhesion and spreading of chinese hamster ovary cells to gel-stated versus liquid-state SLBs tethered with E-cadherins. ${ }^{13}$

In this Chapter, culturing of human articular chondrocytes on SLBs is evaluated as a novel strategy to maintain their native (spheroid) morphology in a 2D cell culture system, indicative of their chondrogenic phenotype. The effect of interligand spacing and SLB fluidity is studied to control cell adhesion and spreading of human articular chondrocytes in order to maintain their phenotype. Furthermore, the SLB fluidity was shown to impact the extent of matrix deposition of murine preosteoblastic cells. To this end, an RGD ligand was synthesized and incorporated in either liquid- or gel-state SLBs and these functional SLBs were then used as a substrate for cell culture.

\subsection{Results and discussion}

\subsubsection{Human primary chondrocytes cultured on SLBs}

Zwitterionic liquid-state SLBs were prepared on glass bottomed well plates that were used for the adhesion and spreading of chondrocytes. The chondrocytes were cultured for one week and inspected using bright field and fluorescence microscopy. A significantly decreased number of cells adhered to the SLBs when compared to the glass control as can be seen in the bright field microscopy images in Figure 5.1. Spreading of chondrocytes was virtually absent on the SLBs and the cells maintained a spheroid morphology. These observations are in agreement with the reported non-fouling nature of zwitterionic SLBs. Despite maintaining their native morphology, a lower cell viability was observed in the case of culturing chondrocytes on unmodified, zwitterionic SLBs when compared to glass controls as detected using a live/dead assay. 


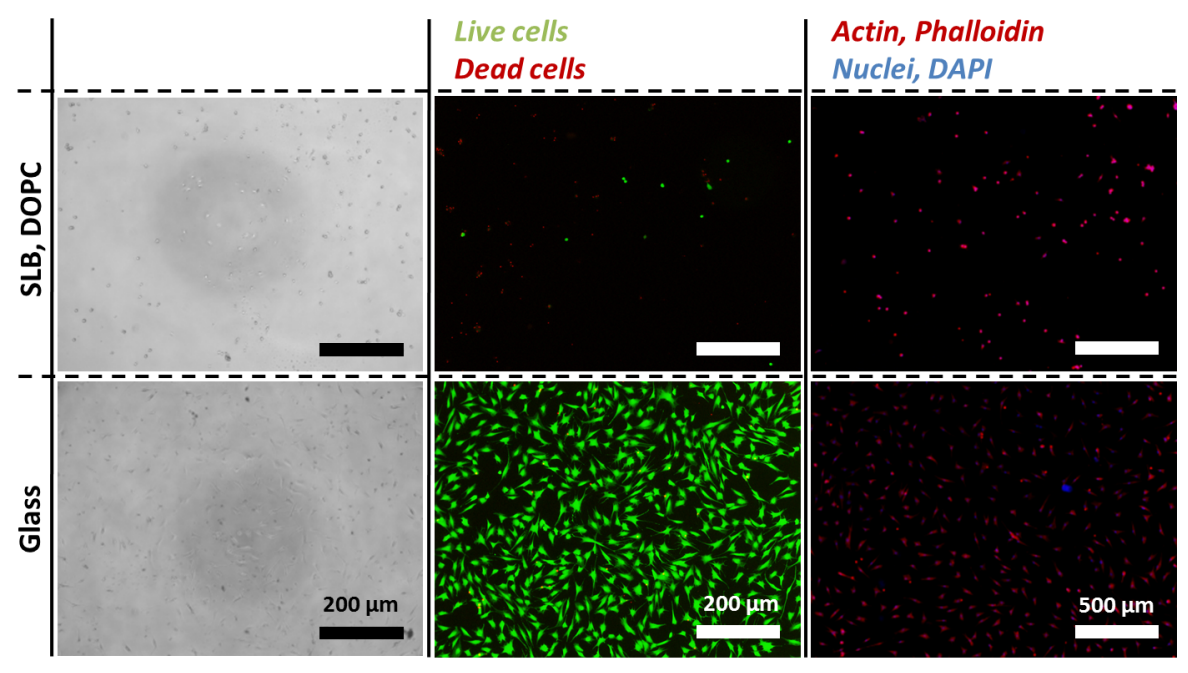

Figure 5.1: Human articular chondrocytes cultured on liquid-state SLBs; human articular chondrocytes were cultured for 1 week on liquid-state SLBs, consisting of DOPC, prepared on glassbottom well plates and on control glass surfaces. Bright field images are shown, cell viability was assessed with a live/dead assay and fluorescence analysis of the cytoskeleton (F-actin) and nucleus was performed.

To specifically promote the adhesion and viability of chondrocytes on zwitterionic SLBS, the presence of an RGD ligand on the SLB would be an interesting strategy to engage with $\alpha_{v} \beta_{3}$ integrin receptors of chondrocytes. ${ }^{14,15}$ To this end, an RGD ligand carrying two palmitoyl chains was synthesized and purified as described in the experimental section. The experimental approach is shown in Figure 5.2. By varying the doping density of the RGD ligand in the vesicles used for the preparation of SLBs, the interligand spacing of RGD ligands in the SLB can be modulated and used as a measure to study the adhesion and spreading of chondrocytes. The interligand spacing in SLBs, defined as $d$ (in $\mathrm{nm}$ ), can be approximated as ${ }^{16}$

$$
d=\sqrt{\frac{A_{\text {Lipid }}}{\chi_{R G D}}}
$$

where $A_{\text {Lipid }}$ the average lipid area in $\mathrm{nm}^{2}$ and $\chi_{R G D}$ molar fraction of palmitoylated RGD. The calculated $d$ assumes equal distribution between the top and bottom leaflet, full incorporation of the amphiphile into the SLB and a homogeneous distribution throughout the bilayer. We aimed for insertion of RGD peptides in the SLB covering $d$-values of 500, 250, 100 and $50 \mathrm{~nm}$. To minimize the effects of cell-cell interactions, low cell seeding densities $\left(5,000\right.$ cells $\left./ \mathrm{cm}^{2}\right)$ were used. 
a)

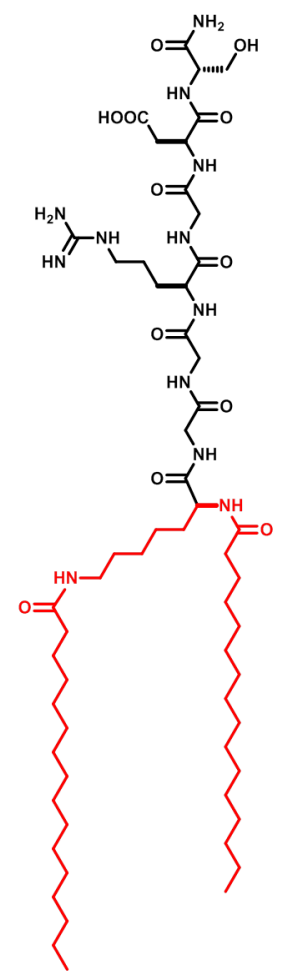

b)
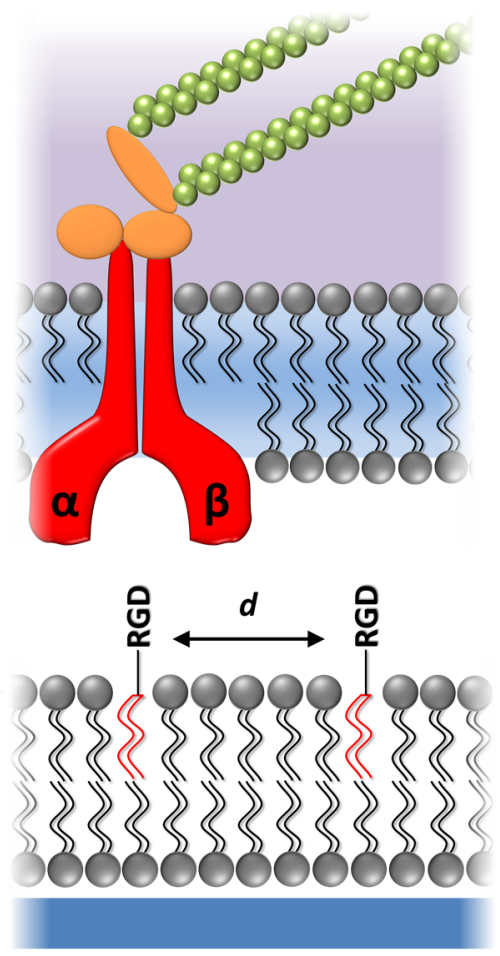

Figure 5.2: Experimental approach for RGD-functionalized SLBs; a) the chemical structure of the RGD peptide carrying two palmitoyl chains. b) Illustration showing interligand spacing (d), integrin engagement (Red) with the SLB and cytoskeletal coupling via focal adhesion molecules (Orange) to actin filaments (Green).

The presence of RGD peptides in the liquid-state DOPC-based SLBs resulted in a higher number of viable adhered cells as was observed in the life/dead assay (Figure 5.3). The highest chondrocyte cell viability was observed for SLBs with $d$-values in the range of 50 $250 \mathrm{~nm}$, while at the lowest amount of inserted RGD peptides a similar viability was observed as in the case of glass surfaces (Figure 5.4). Observations made using bright field microscopy show predominantly spheroid cells throughout the $d$-range that was evaluated, while fluorescence analysis of the cell cytoskeleton revealed a more spread morphology at $d$-values of 100 and $50 \mathrm{~nm}$. This observation was confirmed by cell shape analysis showing a significant increase in eccentricity and a decrease in form factor ${ }^{a}$ of the

\footnotetext{
${ }^{\mathrm{a}} 4 * \pi *$ area/perimeter ${ }^{2}, 1$ equals a perfectly circular object
} 
cell cytoskeleton. This is complemented by a significant increase in projected cell area (Figure 5.4).

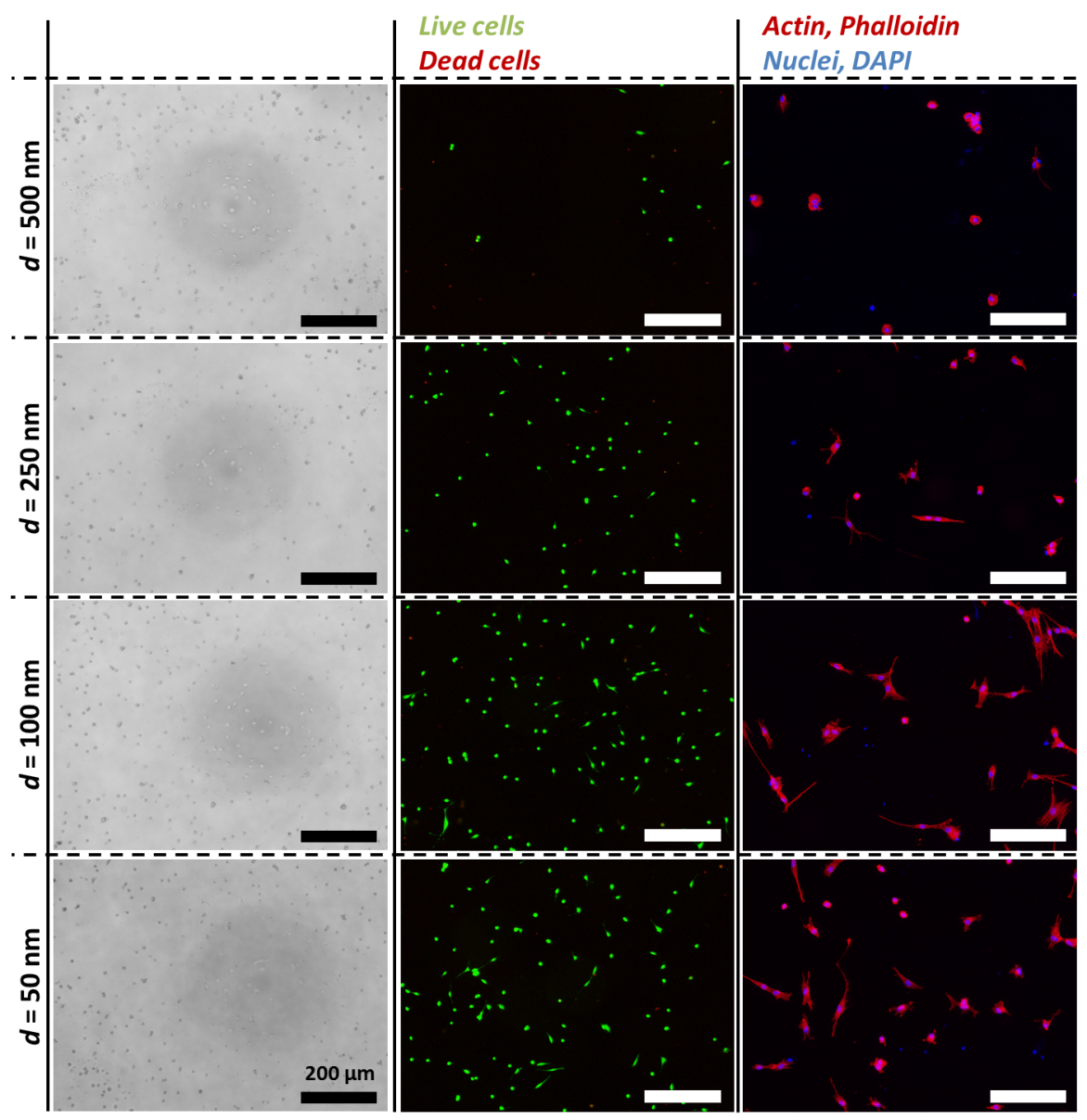

Figure 5.3: Effects of different $d$-values of RGD ligands in liquid-state SLBs on human chondrocytes; human articular chondrocytes were cultured for 1 week on liquid-state SLB, consisting of DOPC, doped with varying amounts of palmitoylated RGD. $d$-values of 50, 100, 250 and $500 \mathrm{~nm}$ were studied. Representative bright field, live/dead staining and fluorescence micrographs of cytoskeleton (F-actin) and nucleus are shown. Scale bar $200 \mu \mathrm{m}$.

The results show that the interligand spacing of cell adhesive RGD sites present on liquidstate SLBs can be used to control the extent of chondrocyte spreading. In particular at a $250 \mathrm{~nm}$ spacing of the RGD peptides, high cell viability was observed without significant chrondrocyte spreading during the course of 1 week. However this is not taken into 
consideration a possible proliferation response. When the amount of RGD ligands on the SLBs result in a spacing of less than $250 \mathrm{~nm}$, a fibroblastic phenotype is observed. This observation is in agreement with literature that describes the adhesion and spreading of chondrocytes on fouling glass surfaces. ${ }^{17}$ When comparing the response of other cell types to changes in RGD density on gold nanodot surfaces, ${ }^{18}$ osteoblasts show differences in their extent of cell spreading and cell polarization as a function of RGD spacing. ${ }^{19}$ In the case of fibroblast cells, cell spreading was observed on RGD surfaces with an interligand spacing of $440 \mathrm{~nm}$ while for focal contact and stress fibre formation an increased RGD surface density of $140 \mathrm{~nm}$ was required. ${ }^{20}$ In the case of mesenchymal stem cells an RGD interligand spacing of 30 and $60 \mathrm{~nm}$ was shown to promote osteogenesis and adipogenesis, respectively. ${ }^{21}$

a)

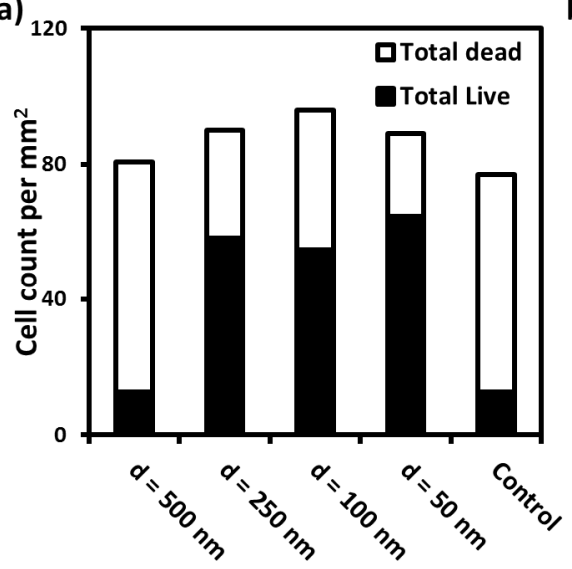

b)

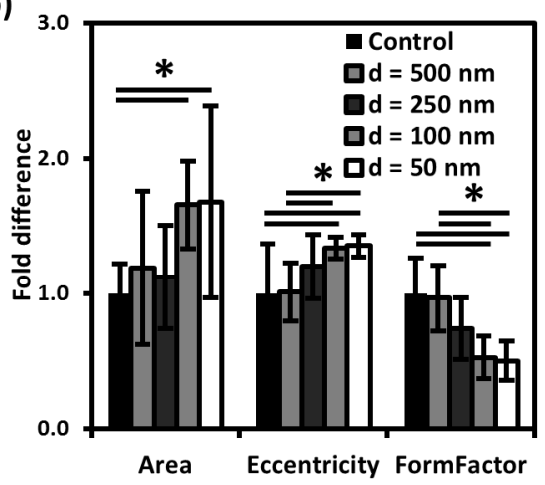

Figure 5.4: Quantified effect of RGD interligand spacing on human chondrocytes; a) result of live/dead assay after 1 week of human articular chondrocytes cell culture on liquid-state SLB doped with RGD and b) quantified cell shape features. $d$-values of 50, 100, 250 and $500 \mathrm{~nm}$ were studied. Data presented as mean \pm SD, 1 -way ANOVA * $p<0.05$, post-hoc Tukey test.

Much to our surprise, when chondrocyte cells were seeded on DPPC-based gel-state SLBs containing similar RGD $d$-values, a higher number of chondrocytes adhered and the cell viability was higher when compared to the observations made on the DOPC liquid-state SLBs after 1 week of culture (Figure 5.5 and 5.6). Upon increasing the RGD-doping density, the chondrocyte cell layer morphology dramatically changed from predominantly single cells at high $d$-value $(500 \mathrm{~nm})$ to clusters of cells at low $d$-value $(50 \mathrm{~nm})$. These clusters of cells consisted of viable cells as concluded from a live/dead staining (Figure 5.5). The 
presence of clusters of cells was also clearly observed with bright field and fluorescence microscopy.

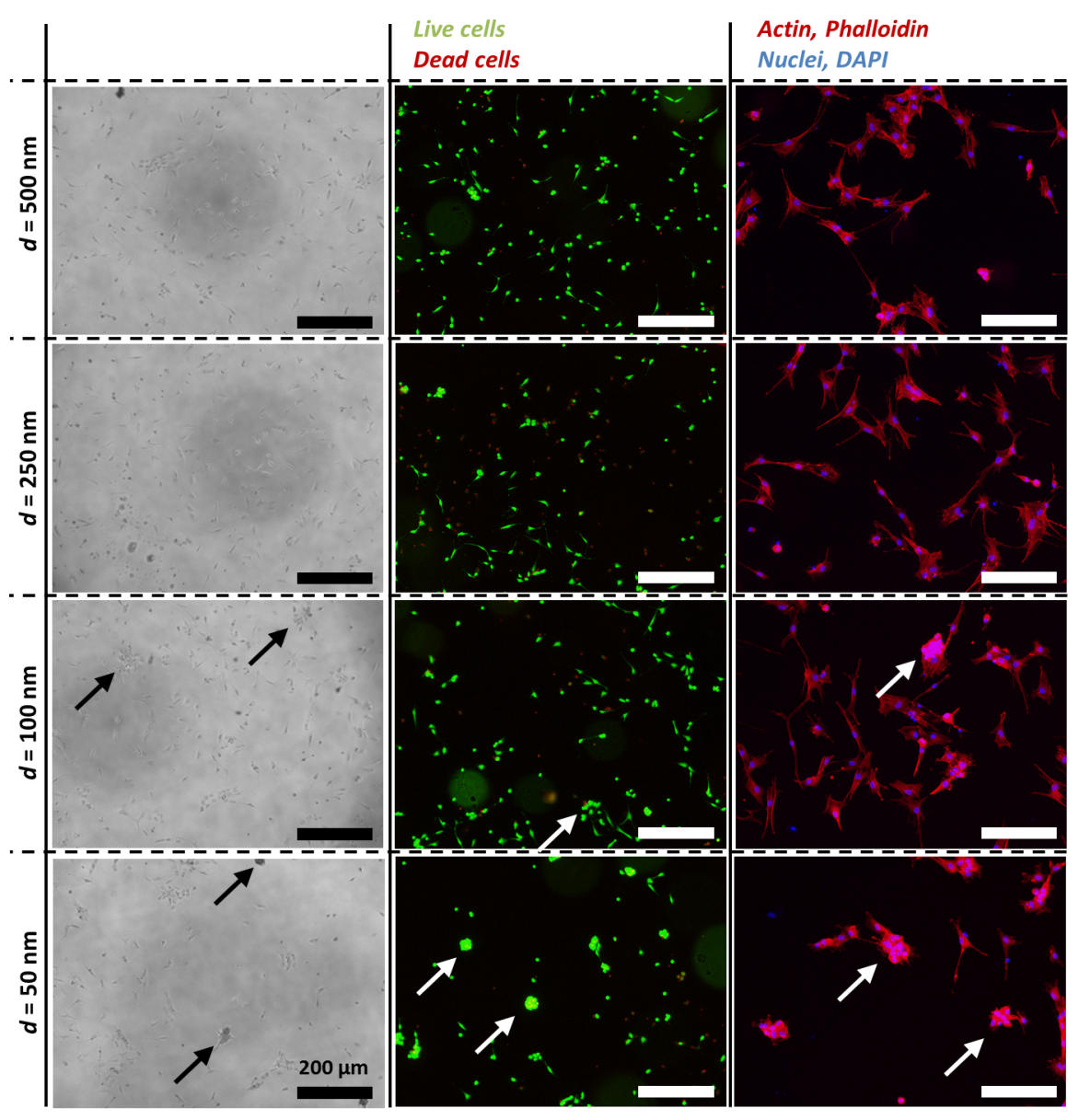

Figure 5.5: Effect of different $d$-values RGD amphiphile in gel-state SLB on human chondrocytes; human articular chondrocytes were cultured for 1 week on liquid-state SLB, consisting of DPPC, doped with varying amounts of palmitoylated RGD. $d$-values of 50, 100, 250 and $500 \mathrm{~nm}$ were studied. Representative bright field, live/dead staining and fluorescence micrographs are shown. Arrows indicate cell aggregates. Arrows indicate cell clusters. 


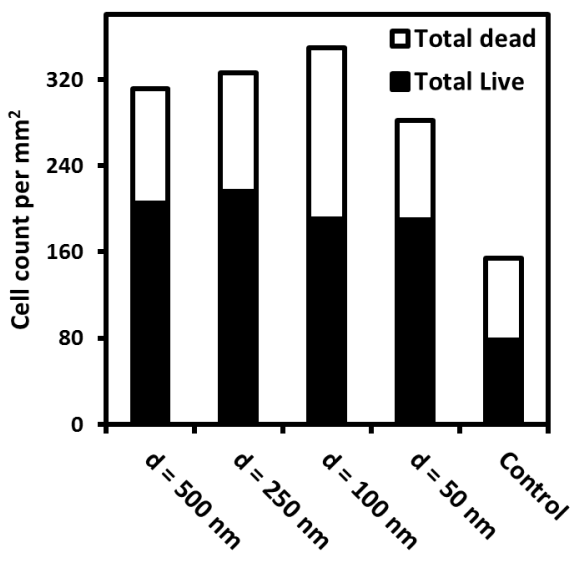

Figure 5.6: Results of live/dead assays on gel-state SLBs; live/dead after 1 week of human articular chondrocytes cell culture on gel-state SLB doped with RGD, d-values of 50, 100, 250 and $500 \mathrm{~nm}$ were used.

To carefully examine the effect of the differences between the mobility of the RGD ligands in the liquid-state versus gel-state SLBs on the cytoskeletal organization of human chondrocytes, focal adhesion (FA) and stress fibre formation was evaluated. To this end, chondrocytes were seeded on SLB surfaces to display a similar projected cell area and cell layer morphology. When chondrocytes were cultured on liquid- and gel-state SLBs doped with RGD ligands at a $d$-value of $5 \mathrm{~nm}$, on both types of SLBs clusters of cells were observed and single cells spread with qualitatively similar cell area and cell viability (Figure S1). A more in depth investigation into cytoskeletal organization was performed for single spread chondrocytes on liquid- and gel-state SLBs functionalized with a $5 \mathrm{~nm}$ interligand spacing of RGD (Figure 5.7). Cells grown on the liquid-state SLBs showed a largely disorganized actin network in absence of large FA assemblies, cells grown on gel-state SLBs had well-defined actin stress fibres and large FA assemblies that were located at the cell periphery. These results indicate that changing the mobility of RGD ligands in SLBs, stress fibre formation and focal adhesion assembly in human chondrocytes can be modulated. As a result, a more stable cell attachment on gel-state SLBs is suggested. Although it has been suggested that initial RGD-integrin engagement and clustering is independent of traction forces, ${ }^{22,23}$ our results are in agreement with observations that show that contraction-mediated maturation of these integrin clusters are required for stable adhesion. ${ }^{24}$ Our observations are also in line with previous discussions that liquidstate SLBs bearing mobile adhesive sites can impair generation of traction forces by cells. ${ }^{10}$ As a result, the physical properties of the SLB can be sensed differentially by cells and can 
modulate the assembly of the actomyosin cytoskeletal network such as stress fibre formation in human chondrocytes. ${ }^{25}$

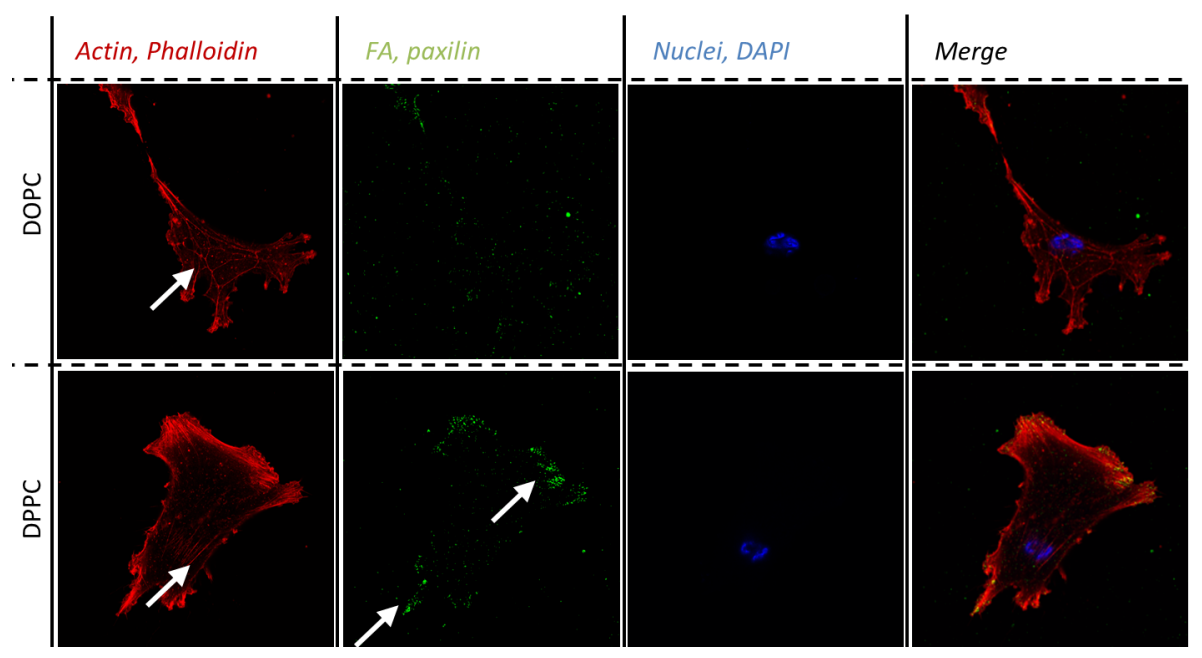

Figure 5.7: FA and stress fibre formation in chondrocytes cultured on liquid vs. gel-state RGD-SLBs; human articular chondrocytes were cultured for 48 hours on liquid- and gel-state SLBs, consisting of DOPC and DPPC, respectively. Fluorescence analysis of the cytoskeleton (F-actin), nucleus and FA (paxilin) were performed. Palmitoylated RGD was added at $d=5 \mathrm{~nm}$. Arrows highlight actin filaments and FAs.

\subsubsection{KS483 matrix deposition}

Motivated by our findings that the physical properties of the SLB can be sensed differentially by chondrocytes as detected in the assembly of the actomyosin cytoskeletal network such as stress fibre formation, we next investigated whether the change in the lateral mobility of the RGD ligands would be sufficient to steer cell differentiation. It has been shown that inhibiting contractility of mesenchymal stem cells by e.g. blebbistatin promotes adipogenesis on otherwise osteogenic surfaces. ${ }^{26}$ Since force generating and, as a consequence, cell contractility can be impaired on liquid-state SLBs, we hypothesized that similar effects would be observed on these mobile layers. To this end, murine preosteoblastic (KS483) cells were cultured on liquid and gel-state SLBs bearing RGD ligands at $d$-values of 25 and $100 \mathrm{~nm}$. KS483 have been used as a model cell line for mesenchymal stem cells that can differentiate into osteoblasts, chondrocytes and adipocytes. The KS483 cells were cultured under either osteogenic or adipogenic conditions at high seeding density of ca. 10,000 cells $/ \mathrm{cm}^{2}$. After two weeks of cell culture the samples were fixed and stained for alkaline phosphatase (ALP) or Oil Red O which are 
markers for osteogenesis and adipogenesis, respectively. The results in Figure 5.6a show that adipogenesis is more evident in the case when KS483 cells were cultured on liquidstate SLBs when compared to observations made when KS483 cells were grown on gelstate SLBs. In contrast, osteogenesis was promoted on gel-state SLBs instead of liquidstate SLBs. Quantification of ALP and Oil Red O (Figure 5.6b) clearly shows this differential behaviour.

a)

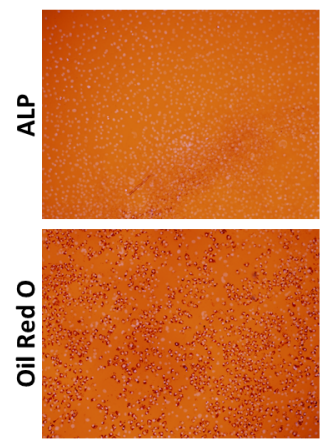

DOPC, $d=25 \mathrm{~nm}$
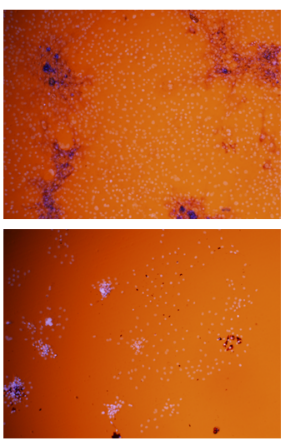

DPPC, $d=25 \mathrm{~nm}$ b)

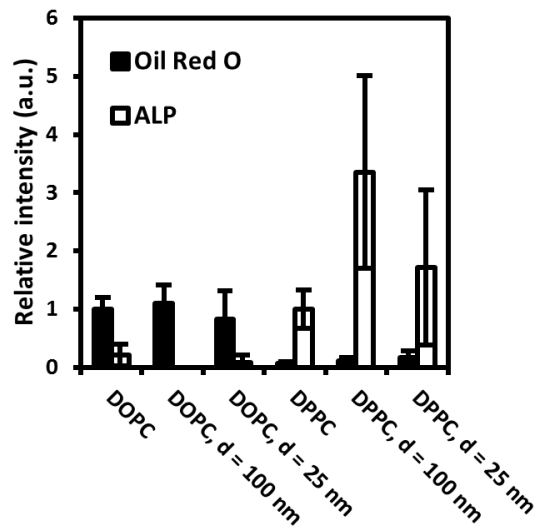

Figure 5.6: Matrix depositions of KS483 cells on SLBs; KS483 cells were cultured for two weeks in osteogenic or adipogenic media. a) Histological staining for ALP (osteogenic medium) and Oil Red O (adipogenic medium) of DOPC and DPPC SLBs doped with $d=25 \mathrm{~nm}$ of RGD and b) quantified ALP and Oil Red $O$ staining for $d$-values of 25 and $100 \mathrm{~nm}$. DAPI was used to counterstain the cell nucleus. Data presented as mean \pm SD.

\subsection{Conclusion}

Successful preparation of zwitterionic liquid- and gel-state SLBs with inserted RGD ligands is described and used to promote cell adhesion. Human chondrocyte cell spreading and cytoskeletal organization was influenced by varying the RGD density. Moreover, we observed that the lateral mobility of the RGD ligands across the SLB strongly influences chondrocyte cell organization. The cell layer morphology ranged from single spheroid cells, single spread cells to the formation of cell clusters. Chondrocyte cell viability could be tuned using liquid-state SLBs by varying the RGD-doping density. Close inspection of the FA and actin fibres at high RGD densities revealed larger FA assemblies on gel-state SLBs with a more organized cell cytoskeleton in comparison with liquid-state SLBs. Moreover, we found that the lateral mobility of the RGD ligands inverted the cell matrix deposition in murine preosteoblastic cells. 


\subsection{Experimental section}

\subsubsection{Materials}

All starting materials and chemicals were purchased from Sigma-Aldrich, Greiner, Fluka, Serva, Avanti Polar Lipids, Gibco, Lonza, Invitrogen, and they were used as received, unless otherwise stated. MilliQ water with a resistivity higher than $18 \mathrm{M} \Omega \mathrm{cm}^{-1}$ was used in all experiments.

Palmitoylated RGD. KGGRGDS was synthesized according to Figure S2. Rink amide resin (50 mmol scale) was used in a microwave solid-phase peptide synthesizer (CEM). Standard amino acid coupling methods from the manufacturer were used. The first amino acid was coupled using a double coupling procedure. After the final Fmoc deprotection step, 10 eq. NHS-palmitate (vide infra) dissolved in $3 \mathrm{~mL} \mathrm{DMF}$ and 1.1 eq. DIPEA were added to the resin. After $5 \mathrm{~h}$, the resin was washed multiple times with DMF and DCM. After addition of TFA/TIS/milliQ (95/2.5/2.5) and a microwave cleavage (40 W) step was performed for 30 minutes while stirring, the peptide was precipitated in cold diethyl ether. RP-HPLC (reverse-phase high pressure liquid chromatography, Waters C18-column) purification of the mono-palmitoylated peptide was performed using a gradient of water/acetonitrile supplemented with $0.1 \%$ TFA. Using ToF-ESI the $[\mathrm{M}+\mathrm{H}]^{+}$and $[\mathrm{M}+\mathrm{H}]^{2+}$ peaks could be identified for the RGD derivative at 913.2 (calc. $[\mathrm{M}+\mathrm{H}]^{+}$913.6) and 457.1, respectively (Figure S3a-b). After lyophilisation, $5 \mathrm{mg}$ mono-palmitoylated peptide was dissolved in 2 $\mathrm{mL} \mathrm{DSMO}$ and to this solution were added 10 eq. NHS-palmitate dissolved in $2 \mathrm{~mL} \mathrm{DMF}$ and 1.1 eq. DIPEA. The reaction was monitored using ToF-ESI and upon disappearance of the mono-palmitoylated peak, which occurred after 4 - 5 hours. The crude was washed several times with diethyl ether to remove unreacted NHS-palmitate. After lyophilisation, ESI-ToF showed $1153.1[\mathrm{M}+\mathrm{H}]^{+}$(calc. 1151.8) (Figure S3c).

NHS-palmitate. NHS-palmitate was synthesized according to a literature procedure (Figure S4a). ${ }^{27}$ In short, palmitic acid $(20 \mathrm{mmol}), \mathrm{NHS}(20 \mathrm{mmol})$ and DCC (20 mmol) were dissolved in $20 \mathrm{~mL}$ THF at $0^{\circ} \mathrm{C}$ for 1 hour and stirred overnight at room temperature. After filtration to remove solids, recrystallization from hot methanol afforded the title product. Characterization of the product was in agreement with literature data. ${ }^{27}$ Characteristic bands in IR: 2,$912 ; 2,848 ; 1,821 ; 1,071 ; 1,784 ; 1,731 ; 1,212 \mathrm{~cm}^{-1}$ (Figure S4b). 


\subsubsection{Methods}

Vesicle preparation. 1,2-Dioleoyl-sn-glycero-3-phosphocholine (DOPC, Avanti Polar Lipids) was stored as a $25 \mathrm{mg} / \mathrm{mL}$ stock solution in chloroform at $-20^{\circ} \mathrm{C}$. 1,2-Dipalmitoyl-snglycero-3-phosphocholine (DPPC, Avanti Polar Lipids) was stored as a $25 \mathrm{mg} / \mathrm{mL}$ stock solution in chloroform at $-20^{\circ} \mathrm{C}$. Desired molar ratios of lipids and palmitoylated peptides were mixed and dried under a flow of nitrogen in a glass vial, and subsequently placed under vacuum for at least 1 hour. The resulting lipid film was re-suspended by vortexing in MilliQ water to form multilamellar vesicles (MLVs) at $1 \mathrm{mg} / \mathrm{mL}$ at $60^{\circ} \mathrm{C}$. The MLV solution was extruded 11 times through a $100 \mathrm{~nm}$ polycarbonate membrane (Avanti Polar Lipids) at $60^{\circ} \mathrm{C}$. The resulting large unilaminar vesicles (LUVs) were characterized by dynamic light scattering (DLS, Nanotrac, Microtrac) and stored until use at $4^{\circ} \mathrm{C}$ for a maximum of two weeks.

SLB formation. SLB formation was achieved by dilution of the LUV solution to $0.5 \mathrm{mg} / \mathrm{mL}$ in phosphate-buffered saline (0.01 M PBS, Gibco, lacking $\mathrm{MgCl}_{2}$ and $\mathrm{CaCl}_{2}$ ). Prior to LUV incubation, the glass-bottom wells plates (Greiner) were flushed briefly with 1x PBS. Afterwards the wells were incubated with the vesicle suspension for at least $30 \mathrm{~min}$ to allow for vesicle adsorption and rupture to occur. Subsequently, the wells were washed with DI and incubated overnight with $0.01 \mathrm{M}$ PBS containing $100 \mathrm{U} / \mathrm{mL}$ Pen/Strep (Gibco). Solution exchange occurred via serial dilution to minimize dehydration damage.

Primary human chondrocyte cell culture. Human primary chondrocytes of passage number 2 were used for the cell experiments. Cells were harvested from knee joints provided by the hospital ZGT Amelo after total knee replacement with agreement of the local ethical committee. 5,000 cells $/ \mathrm{cm}^{2}$ were seeded on the SLBs. Cells were cultured in DMEM (Gibco) supplemented with $0.2 \mathrm{mM}$ ascorbic acid, $50 \mathrm{mg} / \mathrm{mL}$ ITS-premix, 100 $\mathrm{mg} / \mathrm{mL}$ sodium pyruvate and Pen/Strep $100 \mathrm{U}$ and $100 \mathrm{mg} / \mathrm{mL}$, respectively. Media was added and exchanged via serial dilution to prevent dehydration damage to the SLBs.

KS483 mouse progenitor cell culture. Murine mesenchymal progenitor cells KS483 were cultured in $\alpha$-MEM without phenol red (Gibco) supplemented with $10 \%$ FCS (Greiner Bio One), Pen/Strep and glutamax (Gibco). Cells of passage 2 were seeded at a density of ca. 10,000 cells $/ \mathrm{cm}^{2}$ in $\alpha$-MEM without phenol red supplemented with $10 \%$ heat-inactivated FCS, Pen/Strep and glutamax. Medium was refreshed every 3-4 days with $50 \mu \mathrm{g} / \mathrm{mL}$ ascorbic acid (Merck) from day 4 and $5 \mathrm{mM} \beta$-glycerol phosphate (Sigma-Aldrich) from day 11 onwards for osteogenic cultures, while for adipogenic cultures the medium was supplemented with $50 \mu \mathrm{g} / \mathrm{mL}$ ascorbic acid and $50 \mu \mathrm{M}$ Indomethacin (Sigma-Aldrich). 
After 14 days the cell cultures were analysed. Media was added and exchanged via serial dilution to prevent dehydration damage to the SLBs.

Cell analysis. For fluorescent labelling cells were fixed for 15 minutes in $10 \%$ formalin solution (Sigma-Aldrich) and permeabilized for 4 minutes using PBS - 0.1\% Triton X100. DAPI (Invitrogen) and Phalloidin (Invitrogen) were used to visualize the nuclei and actin cytoskeleton, respectively. Phalloidin (1:300 in PBS) was left to incubate for 20 minutes at RT in the dark and counterstained with DAPI (1:100 in PBS) is added and left to incubate for 10 minutes. After each step in the aforementioned procedure the cells were washed with 1x PBS. Paxilin was stained with phos Pax-Y118 (Invitrogen). For live/dead analysis a LIVE/DEAD ${ }^{\circledR}$ Viability kit (Invitrogen) was used according to manufacturer instruction.

Fluorescence microscopy. An Olympus inverted IX71 epi-fluorescence research microscope with a Xenon X-cite $120 \mathrm{PC}$ as light source and a digital Olympus DR70 camera for image acquisition was used to acquire fluorescence micrographs at $10 x$ and $20 x$ magnification. DAPI was imaged using $325 \leq \lambda_{\mathrm{ex}} \leq 375 \mathrm{~nm}$ and $\lambda_{\mathrm{em}}>420 \mathrm{~nm}$, Paxillin was imaged using $460 \leq \lambda_{\mathrm{ex}} \leq 490 \mathrm{~nm}$ and $\lambda_{\mathrm{em}}>525 \mathrm{~nm}$ and Phalloidin-alexa567 was imaged using $510 \leq \lambda_{\mathrm{ex}} \leq 550 \mathrm{~nm}$ and $\lambda_{\mathrm{em}}>590 \mathrm{~nm}$. ISO200 camera setting was used to record high quality, low noise images and care was taken to ensure image acquisition was performed in the linear response regime. High resolution imaging was performed with a Nikon A1 CSLM with an oil-immersion $100 x$ objective.

Histological staining. For Oil Red O staining, cells were fixed in $10 \%$ formalin (SigmaAldrich) for 15 minutes. Samples were washed in DI and incubated for 5 minutes in $60 \%$ isopropanol in DI. Subsequently, filtered Oil Red O solution (Sigma-Aldrich, stock $500 \mathrm{mg}$ in $99 \mathrm{~mL}$ isopropanol and $1 \mathrm{~mL} \mathrm{DI}$, working solution $42 \mathrm{~mL}$ stock $+28 \mathrm{~mL}$ DI) was added and left to incubate for 5 minutes. Afterwards cells were rinsed extensively with DI. For ALP staining, cells were fixed for 1 hour in $10 \%$ formalin and stained using the Alkaline Phosphatase kit (Sigma-Aldrich) according to manufactures instructions. Oil Red O and ALP were imaged with bright field microscopy. In both cases, DAPI was used to counterstain the cell nucleus. ALP and Oil Red O was quantified with CellProfiler. ALP intensity was normalized against DPPC-SLB and Oil Red O against DOPC-SLB.

Data analysis. Image analysis was performed using ImageJ (NIH), Origin (OriginLab) and Excel (Microsoft). Cell shape analysis was performed with CellProfiler. ${ }^{28}$

Statistics. 1-way Anova was used for hypotheses testing. Prior to Anova evaluation normal distribution of the data sets was confirmed by performing the Shaphiro-Wilk test. Grubb's test was used to remove potential outliers and a Post-hoc Tukey's test provided means 
comparison. Equal variance was confirmed by Levene's test. Excel (Microsoft), SPSS (IBM) and Origin (OriginLab) were used for the statistical analysis.

\subsection{References}

Steadman, J. R.; Rodkey, W. G.; Briggs, K. K. J. Knee Surg. 2002, 15, 170-176.

Bartlett, W.; Skinner, J. A.; Gooding, C. R.; Carrington, R. W.; Flanagan, A. M.; Briggs, T. W.; Bentley, G. J. Bone Joint Surg. Br. 2005, 87, 640-645.

Kon, E.; Filardo, G.; Berruto, M.; Benazzo, F.; Zanon, G.; Della Villa, S.; Marcacci, M. Am. J. Sports Med. 2011, 39, 2549-2557.

Demoor, M.; Ollitrault, D.; Gomez-Leduc, T.; Bouyoucef, M.; Hervieu, M.; Fabre, H.; Lafont, J.; Denoix, J. M.; Audigie, F.; Mallein-Gerin, F.; Legendre, F.; Galera, P. BBA. General subjects 2014, 1840, 2414-2440.

Schnabel, M.; Marlovits, S.; Eckhoff, G.; Fichtel, I.; Gotzen, L.; Vecsei, V.; Schlegel, J. Osteoarthr. Cartil. 2002, 10, 62-70.

Watt, F. M. J. Cell Sci. 1988, 89 ( Pt 3), 373-378.

Mukaida, T.; Urabe, K.; Naruse, K.; Aikawa, J.; Katano, M.; Hyon, S. H.; Itoman, M. J. Orthop. Sci. 2005, 10, 521-528.

Johnstone, B.; Hering, T. M.; Caplan, A. I.; Goldberg, V. M.; Yoo, J. U. Exp. Cell Res. 1998, 238, 265-272.

Ananthanarayanan, B.; Little, L.; Schaffer, D. V.; Healy, K. E.; Tirrell, M. Biomaterials 2010, 31, 87068715.

Yu, C.-h.; Rafiq, Nisha Bte M.; Krishnasamy, A.; Hartman, Kevin L.; Jones, Gareth E.; Bershadsky, Alexander D.; Sheetz, Michael P. Cell Reports 2013, 5, 1456-1468.

Sandrin, L.; Coche-Guerente, L.; Bernstein, A.; Basit, H.; Labbe, P.; Dumy, P.; Boturyn, D. Org. Biomol. Chem. 2010, 8, 1531-1534.

Nam, J. M.; Nair, P. M.; Neve, R. M.; Gray, J. W.; Groves, J. T. ChemBioChem 2006, 7, 436-440.

Andreasson-Ochsner, M.; Romano, G.; Hakanson, M.; Smith, M. L.; Leckband, D. E.; Textor, M.; Reimhult, E. Lab Chip 2011, 11, 2876-2883.

Ruoslahti, E.; Pierschbacher, M. D. Cell 1986, 44, 517-518.

Ruoslahti, E. Annu. Rev. Cell Dev. Biol. 1996, 12, 697-715.

Korner, A.; Deichmann, C.; Rossetti, F. F.; Kohler, A.; Konovalov, O. V.; Wedlich, D.; Tanaka, M. Plos One 2013, 8.

Goessler, U. R.; Bugert, P.; Bieback, K.; Sadick, H.; Verse, T.; Baisch, A.; Hormann, K.; Riedel, F. Acta Otolaryngol. 2005, 125, 647-653.

Glass, R.; Moller, M.; Spatz, J. P. Nanotechnology 2003, 14, 1153-1160.

Arnold, M.; Cavalcanti-Adam, E. A.; Glass, R.; Blummel, J.; Eck, W.; Kantlehner, M.; Kessler, H.; Spatz, J. P. ChemPhysChem 2004, 5, 383-388.

Massia, S. P.; Hubbell, J. A. J. Cell Biol. 1991, 114, 1089-1100.

Frith, J. E.; Mills, R. J.; Cooper-White, J. J. J. Cell Sci. 2012, 125, 317-327.

Yu, C. H.; Law, J. B. K.; Suryana, M.; Low, H. Y.; Sheetz, M. P. Proc. Natl. Acad. Sci. USA 2011, 108, 2058520590.

Yu, C. H.; Luo, W. W.; Sheetz, M. P. Cell Adhes. Migr. 2012, 6, 280-284.

Moore, S. W.; Roca-Cusachs, P.; Sheetz, M. P. Dev. Cell 2010, 19, 194-206.

Geiger, B.; Spatz, J. P.; Bershadsky, A. D. Nat. Rev. Mol. Cell Biol. 2009, 10, 21-33.

Kilian, K. A.; Bugarija, B.; Lahn, B. T.; Mrksich, M. Proc. Natl. Acad. Sci. USA 2010, 107, 4872-4877.

Shen, S. I.; Kotamraj, P. R.; Bhattacharya, S.; Li, X.; Jasti, B. R. J. Drug Targeting 2007, 15, 51-58.

Carpenter, A. E.; Jones, T. R.; Lamprecht, M. R.; Clarke, C.; Kang, I. H.; Friman, O.; Guertin, D. A.; Chang, J. H.; Lindquist, R. A.; Moffat, J.; Golland, P.; Sabatini, D. M. Genome Biol. 2006, 7. 


\subsection{Supporting Information}

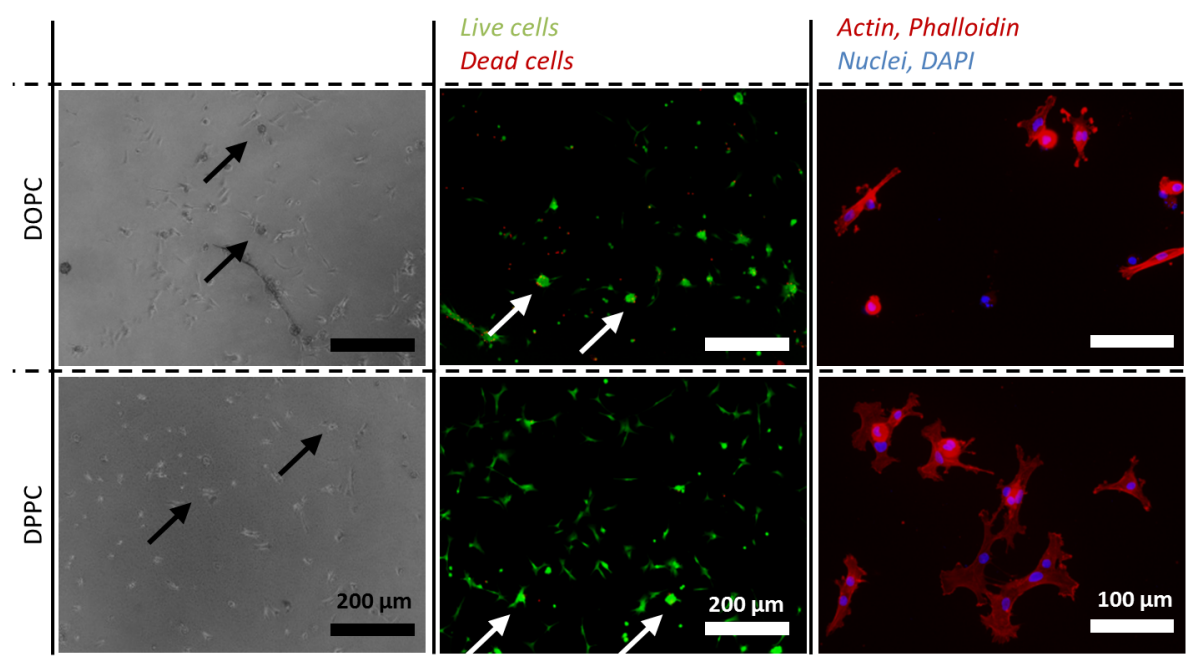

Figure S1: Chondrocytes cultured on liquid- and gel-state SLBs with a $d$-value of $5 \mathrm{~nm}$ of RGD; human articular chondrocytes were cultured for 48 hours on liquid- and gel-state SLBs doped with RGD ( $d$-value of $5 \mathrm{~nm}$ ). Bright field images are shown, cell viability was assessed with a live/dead assay and fluorescence analysis of the cytoskeleton (F-actin) and nucleus was performed. Arrows indicate cell clusters.
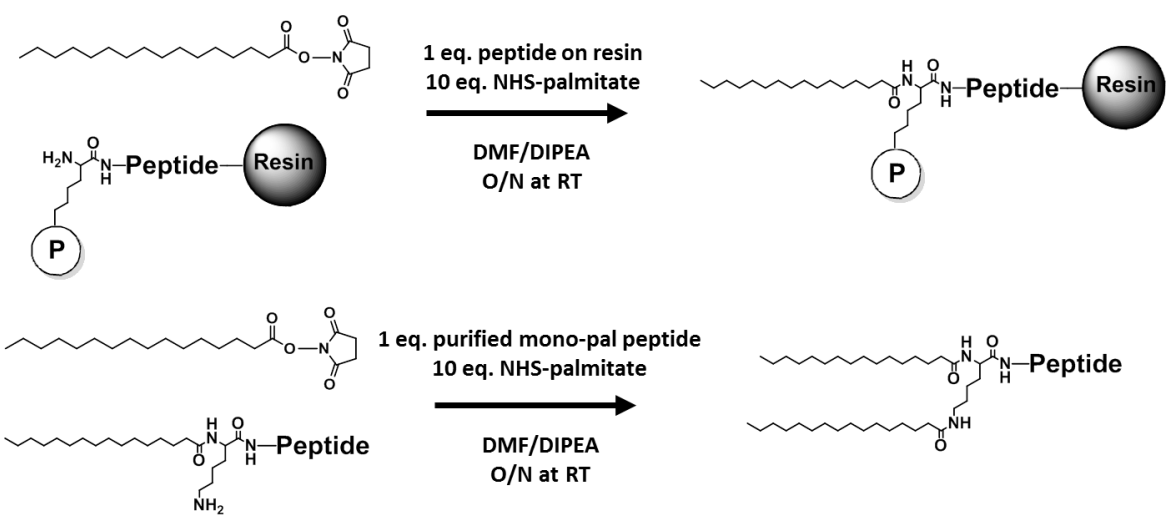

Figure S2: Synthesis of palmitoylated RGD peptides; synthetic route for peptide palmitoylation $(P=$ protecting group). 
a)

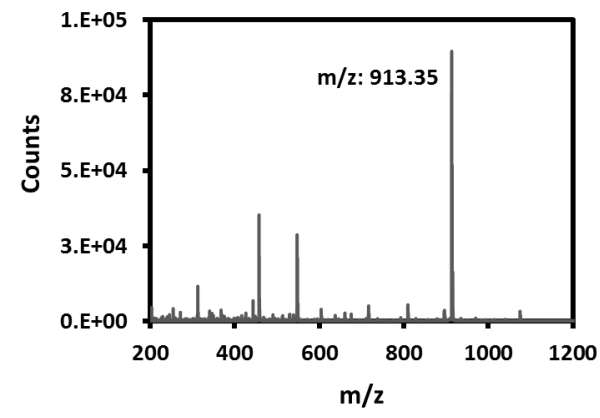

c) b)

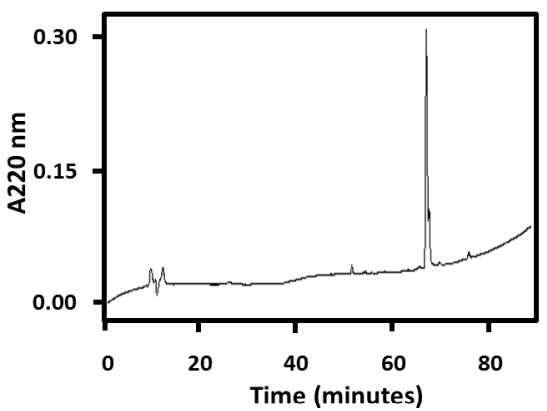

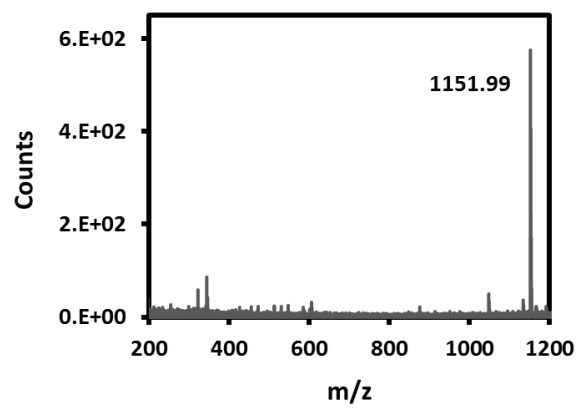

Figure S3: Analysis of palmitoylated peptides; a) ToF-ESI of mono-palmitoylated RGD, b) preparative RP-HPLC chromatogram of mono-palmitoylated RGD and c) ToF-ESI mass of bi-palmitoylated RGD. 
a)
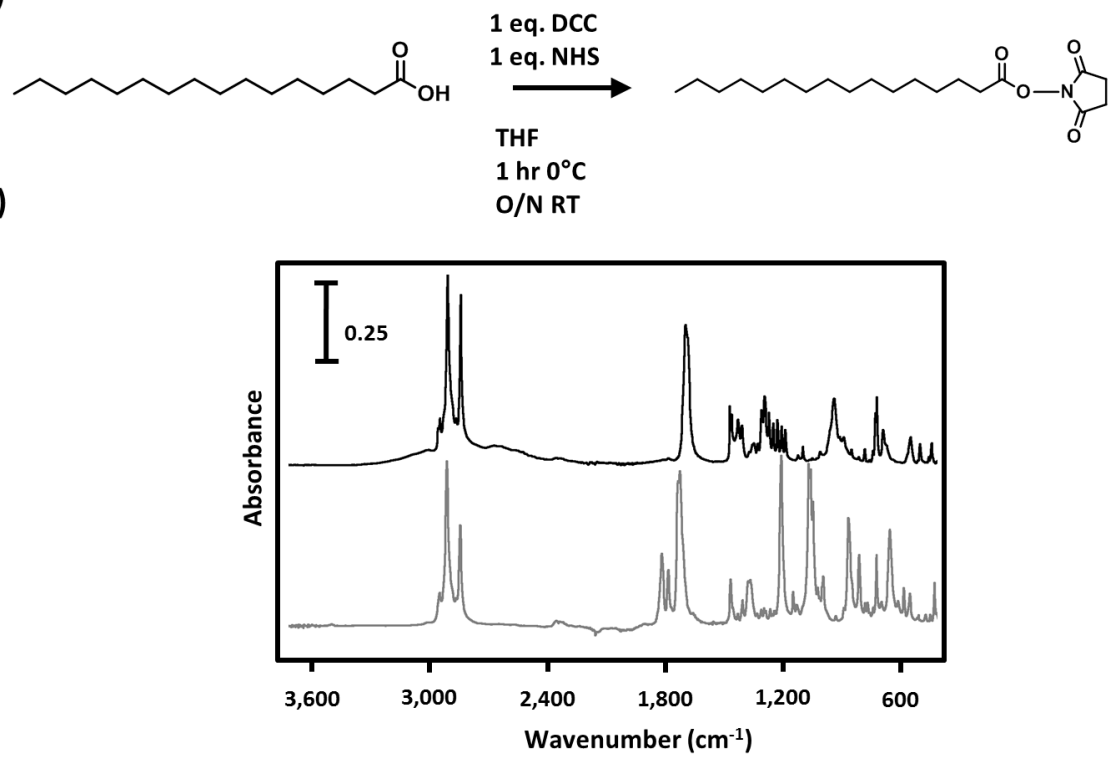

Figure S4: NHS-palmitate synthesis; a) Synthetic route for NHS-palmitate b) FTIR-ATR spectra of recrystallized NHS-palmitate (grey) and palmitic acid (black). 


\section{Chapter 6}

\section{Biomaterial-supported lipid bilayer as a tuneable cell surface}

The field of Tissue Engineering aims at repairing damaged and/or diseased tissue to reconstitute tissue functionality. Polymeric biomaterials used for these purposes have been selected for their biocompatibility and relative inertness in vivo. Although these materials are well suited to provide mechanical support to the tissue formation process, they insufficiently provoke specific biological actions from the host and are susceptible to biofouling, which undermines the tissue formation process. In the present work, supported lipid bilayers (SLBS) are proposed as a means to prevent fouling of biomaterial surfaces and, upon introduction of ligands, to specifically address and steer cells. Our biomaterial supported lipid bilayer (BSLB) relies on universal cholesterol-modification process of biomaterial surfaces for the formation of air-stable SLBs. To this end, the synthesis of a cholesterol linker and the subsequent plasma preconditioning, to allow for cholesterol modification of, various biopolymers is described. BSLBs prepared on cholesterol-modified polycaprolactone (PCL) were shown to be highly non-fouling and could be prepared in $3 D$. The influence of lateral mobility of a cell adhesive RGD ligand allowed us to steer mesenchymal stem cell differentiation to adipogenic and osteogenic lineages. The results presented in this Chapter suggest full mechanical and biological decoupling of the cell response from the underlying support.

\footnotetext{
${ }^{*}$ Manuscript in preparation: J. van Weerd, M. Karperien and P. Jonkheijm
} 


\subsection{Introduction}

In Tissue Engineering the use of biomaterial scaffolds are investigated as templates and supports for tissue formation in vitro and in vivo. Although a relatively new field, examples of reconstituting tissue functionality can be traced back to 2,500 years BC where ox bones were first used as tooth replacements. Although numerous routes for tissue regeneration are currently being studied, a general consensus is the use of inert biomaterials scaffolds. In this case, inertness relates to the biocompatibility of a material e.g. limited immunological responses as well as no apparent cytotoxicity. However, inducing tissue formation and steering cell fate has proven to be a major hurdle caused by a lack of specific biological triggers and the occurrence of non-specific interaction with cells and tissue. In vivo, cellular responses are influenced by a wealth of factors such as the extracellular matrix (ECM), mobile or immobile signalling molecules, cell-cell interaction as well as physical factors in the cell's microenvironment. ${ }^{1-3}$ The plethora of cell interactions affect three biological outcomes, namely tissue formation, tissue homeostasis and tissue regeneration. ${ }^{4}$ Insights into stem cell behaviour highlight key features that need to be taken into consideration when addressing cell fate, that are often inadequate in these artificial systems. Besides the use of ligands, e.g. growth factors, ECM components or peptides, research has shown the importance of the mobility and flexibility of ECM protein chains with which cells interact. ${ }^{5,6}$ This phenomenon, often attributed to stiffness variations in the cell's environment, has been shown to impact mesenchymal stem cell (MSC) differentiation. ${ }^{7}$ In addition, varying only the spacing of ECM epitopes such as the RGD motif influenced MSC focal adhesion formation as well as adipogenic and osteogenic behaviour, in accordance with results described in Chapter $5 .^{8}$

Accounting for all the physiochemical cues present in nature within an artificial system is daunting. Hence, research has often focused on providing cells with a subset of cues sufficient to elicit a certain response. In Tissue Engineering for example, biomaterial scaffolds are designed such that the host serves as a bioreactor for self-repair. ${ }^{9,10}$ Since the cell substrate often perturbs the desired cell response, researchers have suggested materials that enable decoupling of the bulk properties from the local cell microenvironment. ${ }^{11}$ Doing so requires cell cues to be presented on a non-fouling background thereby minimizing non-specific cell responses encountered in in vitro cell culture and upon implantation. ${ }^{12}$ Non-fouling properties are essential since protein adsorption occurs rapidly in a protein-rich environment. Fouling is sometimes exploited to enable cell attachment as a result of protein pre-adsorption, however in vivo the protein ad-layer is known to trigger the immune system and affect the wound healing process. ${ }^{13}$ Material modification with, for example, poly(ethylene oxide) has been used and studied extensively as a way to supress non-specific protein adsorption. ${ }^{14}$ However, the lack of instructive cues in such systems prevents cell adhesion. In one study this has been tackled 
by introducing cell adhesive peptides onto the poly(ethylene oxide) layer. This strategy allows for the preparation of non-fouling electrospun biomimetic fibres able to specifically bind cells. ${ }^{15}$ Hence there is a clear need for an bio-active biomaterial cell interface that is non-fouling and is able to present biological triggers. Since cell-material interactions are mediated by the cell membrane, ${ }^{2}$ a mimetic membrane coating was envisioned to steer cell fate. The development of such a mimetic membrane coating will be described in greater detail.

In this Chapter, we propose a mild and universal strategy to render the surface of any biomaterial non-fouling while providing a high degree of modularity towards tweaking the bioactivity of the surface of the biomaterial. Our strategy is based on the use of supported lipid bilayers (SLBs) that are widely recognized as suitable in vitro cell membrane models for cell-cell interactions and cell-ECM interactions (Chapter 1), however up to now SLBs have not been explored as a surface modification strategy for biomaterials. The limited stability of traditional SLBs in air hampers their use and must be resolved when considering an in vivo application. Once addressed, the hydrated phosphocholine headgroup of natural lipids that are employed to generate SLBs provide the desired non-fouling properties while ligands can be conveniently tethered to SLBs to render them suitable for cell-adhesion. Another unique feature of SLBs is the modularity of the lateral mobility of the lipids and tethered components by careful variations in the alkyl chain length and their degree of saturation and branching. This has been shown to impact matrix deposition of murine preosteoblastic cells and cytoskeletal organization in human chondrocytes (Chapter 5).

\subsection{Results and Discussion}

An overview of our proposed strategy to modify biomaterials with SLBs is given in Figure 6.1 and takes into consideration a way to improve the air-stability of SLBs. A cholesterol linker was covalently attached to the surface of the biomaterial to tightly knit the lipid bilayer to the biomaterial surface as above a certain density of cholesteryl groups on glass, 1:6 ratio of cholesteryl to lipids, air-stable bilayers were reported that remained fluidic even after several dehydration cycles. ${ }^{16}$ Adopting this approach, the fluidity of both the top and the bottom leaflets can be ensured while not affecting the non-fouling behaviour of the lipid bilayer. 


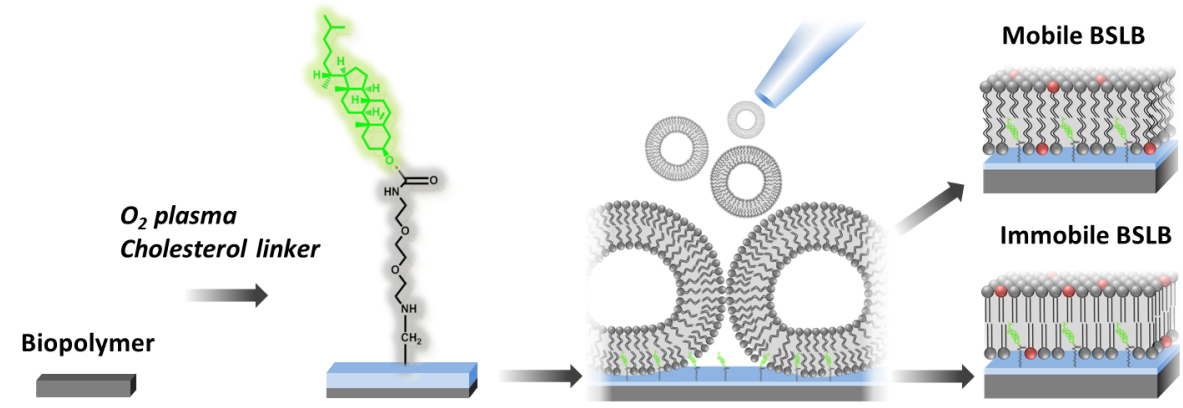

Figure 6.1: overview of the proposed material modification

\subsubsection{Biomaterial activation}

We sought a method that only temporarily introduces functional groups to the outer few nanometers of the biomaterial irrespective of the type of the biopolymer. Restricted modification to only the outer surface is required to retain the bulk polymer properties such as degradation and the mechanical properties, which are strictly required for device performance. Modifying the surface only to present functional groups having a restricted life time is desirable to minimize unwanted side-effects of unconverted functional groups. Therefore, we have selected oxygen plasma (OP) treatment which is known to only alter the surface of biopolymers. In addition, by carefully tuning the OP treatment the formation of carboxylic acid groups can be avoided and mostly aldehyde groups can be introduced. ${ }^{17}$ Aldehydes do not need another chemical activation step in contrast with acid groups, ${ }^{18}$ but readily react with amines yielding imines that subsequently can be reduced to hydrolytically stable secondary amines. ${ }^{19}$ Unreacted aldehydes will be reduced to bio-inert hydroxyl groups in contrast to unconverted chemically activated acid groups that remain prone to react. Brief OP treatment (10 seconds at 40 watts, 200 mTorr) proved effective in generating reactive aldehydes on the different biopolymers such as polycaprolactone (PCL, Figure 6.2), a segmented block copolymer of poly(ethylene oxide terephthalate)/poly(butylene terephtalate) in 1000/70/30 PEOT/PBT ratio (PEOT-PBT, Figure S1) and a block copolymer of polylactic acid and $\mathrm{PCL}$ in 65/35 ratio (PLA-PCL, Figure S2). In all cases, a colorimetric assay against aldehydes was positive after the OP treatment (Figures 6.2a, S1a and S2a), while X-Ray photoelectron spectroscopy (XPS) spectra showed an increased intensity of oxygen containing groups such as carbonyls i.e. $\mathrm{O}-\mathrm{C}=\mathrm{O}$ and $-\mathrm{C}=\mathrm{O}$ (Figures $6.2 \mathrm{~b}-\mathrm{d}, \mathrm{S} 1 \mathrm{~b}-\mathrm{d}$ and $\mathrm{S} 2 \mathrm{~b}-\mathrm{d}$ ). By exploiting the differences in sampling depth of two IR techniques, FT-IR ATR and PM-IRRAS, surface confinement of the modification was evaluated. In the case of FT-IR-ATR this is ca. $5 \mu \mathrm{m}$ for the ATR diamond crystal and several nanometers for PM-IRRAS. After OP treatment, the PM-IRRAS spectra (Figures 6.2e, S1e and S2e) revealed a shoulder in the carbonyl region (ca. 1,750 $\mathrm{cm}^{-1}$ ) 
a)

c)

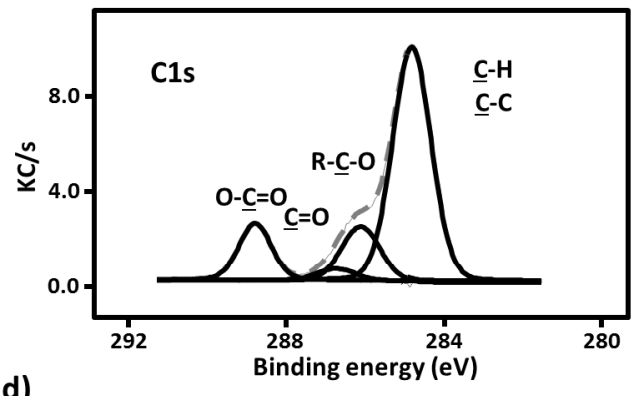

d)

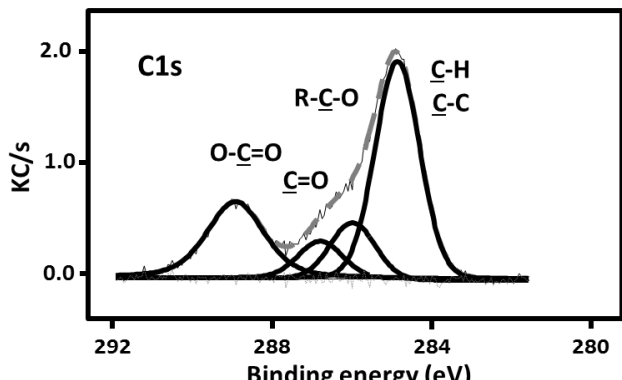

e)

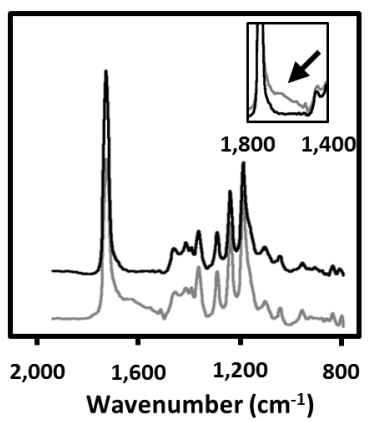

b)
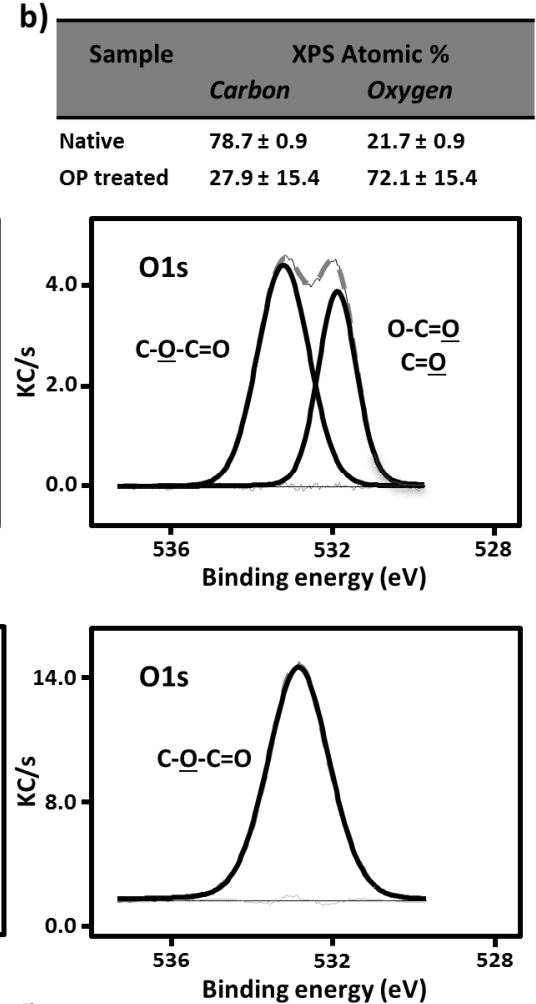

f)

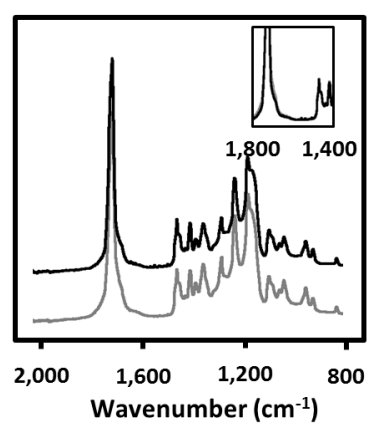

Figure 6.2: Generating aldehyde groups on PCL by an OP treatment; a) results of Purpald reaction to stain aldehydes at the surface of the OP treated polymer samples. The atomic composition given in b) was determined by XPS, mean \pm SD $(n=5)$. XPS spectra of $c) C 1 s$ and O1s of the native PCL and d) OP treated PCL. IR-spectra obtained with e) PM-IRRAS and f) FTIR-ATR of native (Black) and OP treated (Grey) PCL samples. Arrow indicates shoulder in the carbonyl region. 
which was not detected in the ATR spectra (Figure 6.2f, S1f and S2f). Furthermore, comparison of the PM-IRRAS spectra before and after reduction of OP treated samples confirmed the presence of aldehyde groups on the polymer surface. This is indicated by the disappearance of the shoulder in the carbonyl region after reduction of OP treated samples (Figure S3). Taken together, these comparative IR studies confirmed the surface confinement of the modification of the biomaterials using an OP treatment.

To demonstrate the reactivity of the aldehyde groups generated using an OP treatment, reactive micro contact printing of a fluorescent dye bearing a free amine was performed on the aldehyde-modified biopolymers. The resulting prints were investigated using epifluoresence microscopy. On all OP treated biopolymer samples the fluorescent patterns remained visible after stringent washings (Figures 6.3 and S4) while without such an OP treatment the printed rhodamine patterns were washed away in methanol in a 5 minute sonication step. These results confirm the immediate reactivity of aldehyde-functionalized polymers with amine-bearing molecules in agreement with previous research that reported the formation of imines via reactive micro contact printing of lissamine rhodamine $B$ ethylenediamine on aldehyde self-assembled monolayers(SAMs). ${ }^{20}$
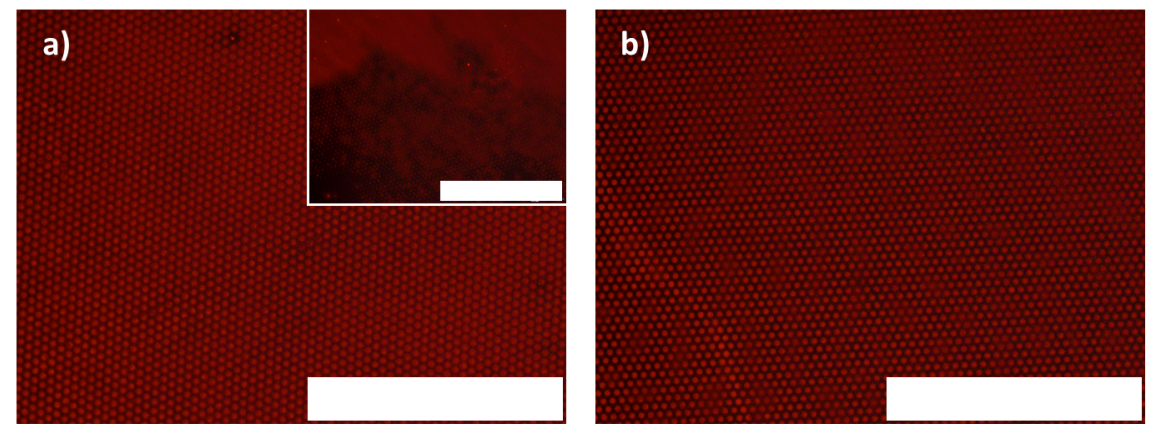

Figure 6.3: Micro-contact printing of rhodamine $B$ ethylenediamine on OP treated PCL samples; OP treated PCL samples a) directly after printing or $\mathbf{b}$ ) following an extensive washing step in methanol. Inset shows native PCL (non-OP treated $\mathrm{PCL}$ ) directly after printing, here no patterns was visible after washing. Scale bar $400 \mu \mathrm{m}$.

\subsubsection{Biomaterial SLB formation}

To surface-tether the lipid bilayer, a cholesterol was functionalized with a hydrophilic amino triethylene glycol spacer and used to react with the aldehyde-modified polymeric surfaces (Figure 6.4a). A description of the synthesis, purification and characterization can be found in the experimental section. The reaction between the cholesterol linker and aldehyde-modified PCL, PEOT-PBT and PLA-PCL polymers was monitored by measuring the 
change in water contact angle (Figure S5). In general, the water contact angle measurements showed that after OP treatment the samples became more hydrophilic while a more hydrophobic surface was observed after coupling of the hydrophobic cholesterol linker. During these experiments, all samples were measured at the same interval after OP treatment, ensuring hydrophobic recovery affected the samples equally. Subsequent reduction facilitated stable bond formation and the conversion of unreacted aldehyde to hydroxyl groups. In this case no effect of reduction on water contact angle was observed, Figure 6.4b. The presence of the cholesterol linker on PCL was confirmed by using time-of-flight secondary ion mass spectrometry (ToF-SIMS), see Figure 6.4c. The molecular ion peak and mass abducts of the linker could be found on PCL-modified surfaces.

a)

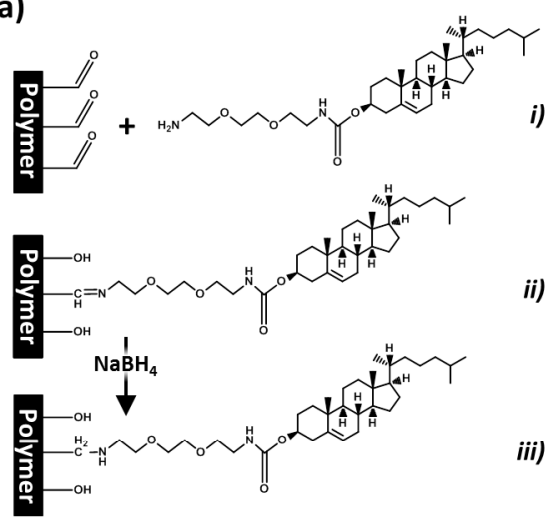

c) b)

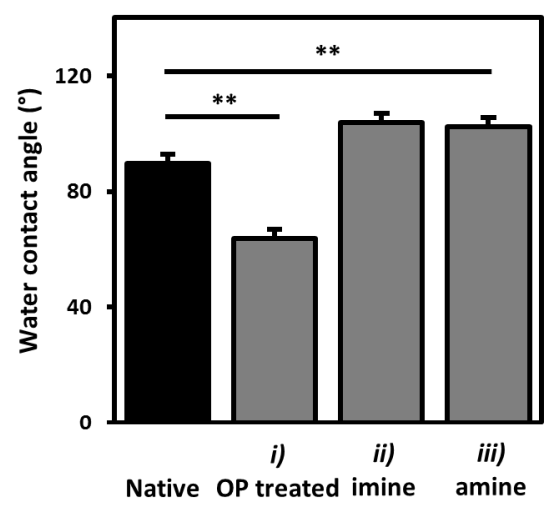

Native OP treated imine amine
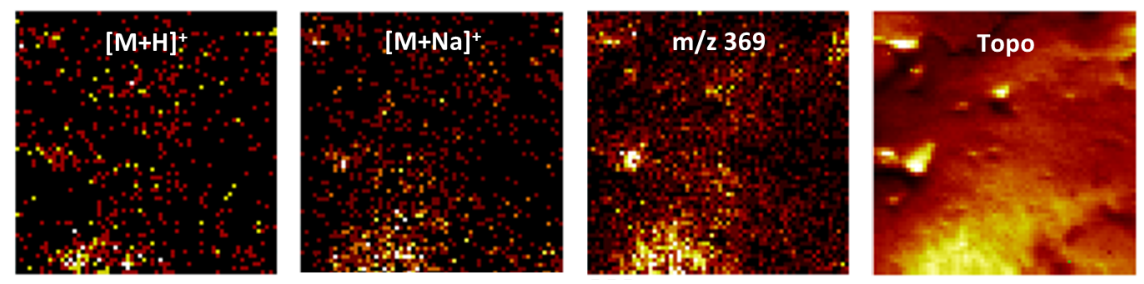

Figure 6.4: Fabrication of cholesterol-modified biopolymers; a) coupling scheme of the cholesterol linker to aldehyde groups on biopolymers introduced with OP treatment. Analysis of modified PCL is shown, namely b) the sensile water contact angle observed during PCL modification, and c) ToFSIMS analysis. $[\mathrm{M}+\mathrm{H}]^{+},[\mathrm{M}+\mathrm{Na}]^{+}$and a $\mathrm{m} / \mathrm{z} 369$ mass abduct of the cholesterol linker were detected on cholesterol-modified PCL. Data presented as mean \pm SD $(n=3)$, Student T-test $* * p<0.01$. 
The next step involves the formation of an SLB on the cholesterol-modified polymer surfaces. Although SLBs can be prepared via the Langmuir-Blodgett method, vesicle fusion or a combination of both techniques, ${ }^{21}$ vesicle fusion allows for easy scaling to the sample size and shape. Large unilaminar vesicles (LUVs) of $100 \mathrm{~nm}$ consisting of neutral, zwitterionic DOPC or DPPC lipids were used to prepare a lipid bilayer on the cholesterolmodified PCL that we abbreviated as biomaterial supported lipid bilayers (BSLBS). Under hypertonic conditions for the LUVs in $1 x$ PBS (phosphate buffered saline, lacking $\mathrm{MgCl}_{2}$ and $\mathrm{CaCl}_{2}$ ) BSLBs formed readily (in ca. 30 minutes) on both OP treated and cholesterolmodified PCL samples. After formation of a bilayer on PCL, the BSLB was inspected by force spectroscopy (Figure 6.5a-b). Both DOPC (Figure 6.5a) and DPPC-based (Figure 6.5b) BSLBs had thicknesses corresponding to a lipid bilayer, 4.1 and $4.8 \mathrm{~nm}$ respectively. ${ }^{22}$

To promote vesicle rupture a freezing cycle was introduced after vesicle adsorption to the cholesterol-modified polymer, ${ }^{23}$ however fluorescence recovery after photobleaching (FRAP) experiments showed similar results. The $D$-values estimated from FRAP measurements were found to decline from $1.0 \pm 0.2$ to $0.55 \pm 0.04 \mu \mathrm{m}^{2} / \mathrm{s}$ for the mobile BSLBs depending on the reaction time between the amino-functionalized cholesterol and the aldehyde-functionalized polymer film indicating that the surface coverage of cholesterol plays a role in the mobility of molecules in the bilayer (Figure 6.5c). In all cases, the mobile fraction was however over $90 \%$ indicating that both leaflets are mobile. Most likely, the SLB on the cholesterol-modified PCL conforms better with the surface than noncholesterol OP treated samples guided by incorporation of cholesterol anchors in the bilayer during the SLB formation. Due to the inherent roughness of the solvent-cast films, the apparent $D$-value will then be reduced, since calculation of $D$-values assumes a 2D flat film. ${ }^{24}$ The non-fouling nature of BSLBs was confirmed by an significant reduction in protein adsorption of $>99 \%$ compared to that for native $P C L$ e.g. $0.27 \pm 0.08 \mathrm{ng} \mathrm{BSA} / \mathrm{cm}^{2}$. When the samples had been allowed to react for 1 hour with the cholesterol linker, dehydration of the samples did not impair the non-fouling behaviour, confirming sufficient air stability of the BSLBs (Figure 6.5d). In addition, the BSLBs still displayed long-range lipid mobility after dehydration (Figure S6). The same procedure to form SLBs on 2D solvent cast polymer sheets was also applied on commercially available 3D PCL scaffolds in order to assess the bilayer formation on more complicated shapes. The lipid bilayer on the 3D scaffold was analysed using two-photon fluorescence microscopy (Figure S7). Fluorescence of the 0.2 mol\% OG-DHPE doped BSLB was detected on the PCL fibres deep within the scaffold (Figure 6.5e) and showed a uniform fluorescence. FRAP measurements on one of the PCL fibres of the construct showed that the fluorescence recovers with characteristic $D$-values equal to the ones estimated for 2D sheets. Although the BSLB appears uniform and intact, the presence of micro- or nanoscopic defects in the layer can not be excluded. 
a)

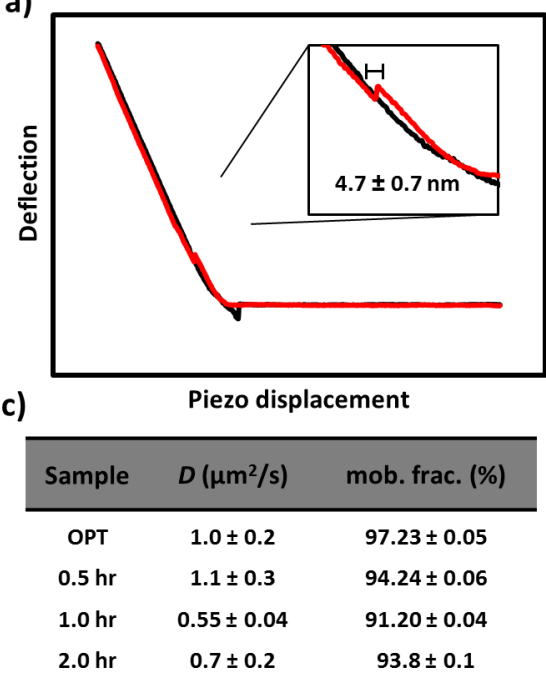

e)

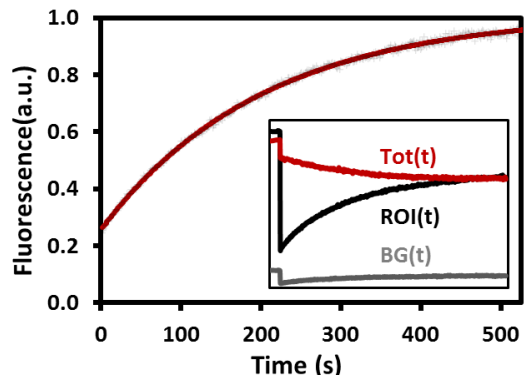

b)
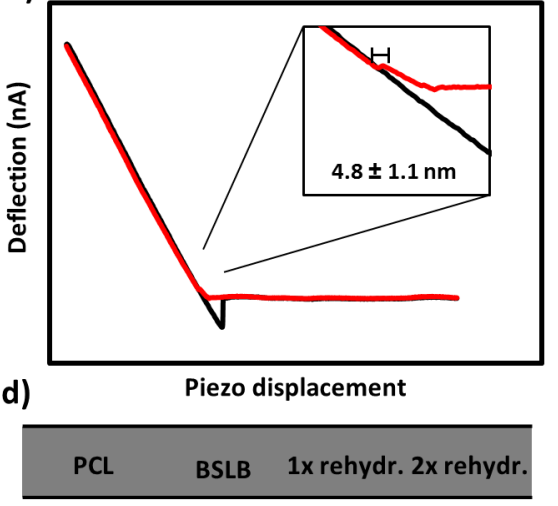

$38 \pm 20 \quad 0.27 \pm 0.08 \quad 0.17 \pm 0.040 .11 \pm 0.01$

(in $\mathrm{ng} / \mathrm{cm}^{2} \mathrm{BSA}$ )
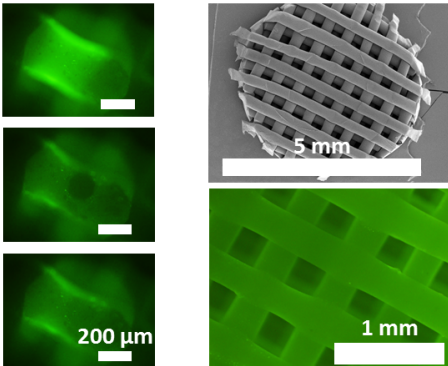

Figure 6.5: Biomaterial SLB characterization; examples of force spectroscopy curve obtained of a) liquid- and b) gel-state BSLBs. The approach (red) and retract (black) curves are given. The breakthrough event is shown in the insets and the mean breakthrough distance is presented as mean $\pm S D(n>10)$. c) Effect of cholesterol linker incubation on $D$-value. Data is presented as mean \pm $\mathrm{SD}(\mathrm{n}>2)$. d) BSA adsorption is presented as mean \pm SD. Student $T$ test, $p<0.05(n=3)$. e) Example of a liquid-state BSLB prepared on a 3D PCL scaffold. The SLB was doped with $0.2 \mathrm{~mol} \%$ of OG-DHPE. A FRAP recovery curve, fluorescence micrographs and electron microscopy image of the 3D PCL scaffold is presented. 


\subsubsection{Mesenchymal stem cell culture on BSLBs}

Our BSLB provides a cell interface that shows little to no protein adsorption. As a consequence, cell fate would predominantly be guided by the insertion of bioactive ligands into the lipid bilayer on the biopolymer and their lateral mobility. The importance of the mobility of SLB-associated E-cadherin ligands had decisive effects on cell spreading and actin fibre arrangement as was previously observed in the case of chinese hamster ovary cells $(\mathrm{CHO})$ cultured on mobile DOPC and immobile MPPC SLBs. ${ }^{25}$ Similarly, in Chapter 5 we have shown that the lateral mobility of cell adhesive sites affects cytoskeletal organization in human chondrocytes and the extent of matrix deposition of murine preosteoblastic cells cultured on SLBs. To bio-activate the BSLB, palmitoylated RGD peptides were synthesized and after purification included in LUVs for preparation of BSLBS (for details regarding the synthesis and purification consult Chapter 5). Such fibronectinderived RGD peptides were used to promote cell adhesion to the otherwise non-fouling BSLBs. ${ }^{26}$ These palmitoylated RGD peptides were embedded in either mobile (liquid-state DOPC) or immobile (gel-state DPPC) BSLBs to study the influence of peptide lateral mobility on MSC differentiation. MSCs can be obtained from bone marrow aspirates and have the potential to differentiate into bone (osteogenesis), cartilage (chondrogenesis) and fat (adipogenesis) tissue, and therefore have been invaluable for tissue regeneration strategies as a cell source. ${ }^{27}$ In this study, immortalized MSCs (iMSCs) were used. These iMSCs were generated from stably transfected human MSCs with the gene for the catalytic subunit for human telomerase. The iMSCs do not show senescence-associated proliferation arrest and retain MSC differentiation potential. ${ }^{28}$ Initial iMSC adhesion was studied after 2 hours of cell seeding in the presence of serum (Figure 6.6). As expected, on both mobile and immobile BSLBs a reduced cell adhesion was observed on the recorded fluorescent micrographs after staining for actin filaments. An average ten-fold reduction in cell count was observed when compared to the cholesterol-modified PCL polymer (Figure 6.6b). Occasionally, cells do adhere and spread, which may be related to defects in the BSLB surface that presumably is the underlying PCL linker surface. Following introduction of palmitoylated RGD peptides to the BSLBs, the cell count increased for both mobile and immobile BSLBs by an average of ca 26-fold when compared to the bare BSLBs while a ca. three-fold increase was observed when compared to that for the cholesterol-modified PCL samples (Figure 6.6a-b). In the case of culturing iMSCs for only 2 hours on immobile RGD modified BSLBs, a significant increase in cell area of ca. $300 \mu \mathrm{m}^{2}$ was noted when compared to their mobile counterparts (Figure 6.6a-b). From the analysis of the cell shape features (Figure 6.6c), a more elongated cell morphology, as indicated by the form factor, is evident on BSLBs that display the palmitoylated RGD peptides. This effect was most pronounced on immobile BSLBs, $0.72 \pm 0.01$ vs. $0.59 \pm 0.01$, which is indicative of variations in the traction forces the cells were able to exert on the different BSLBs. 
a)
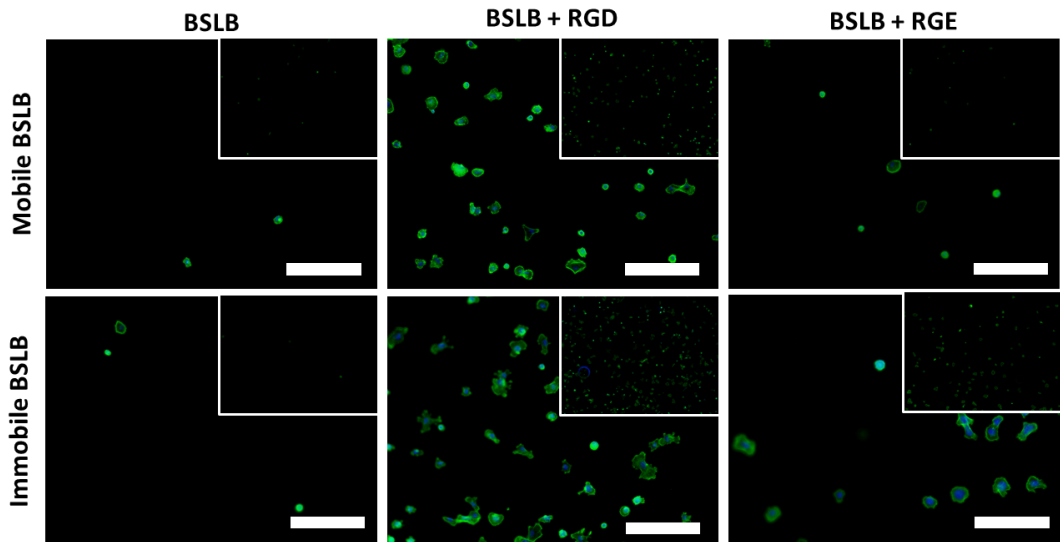

b)

c)
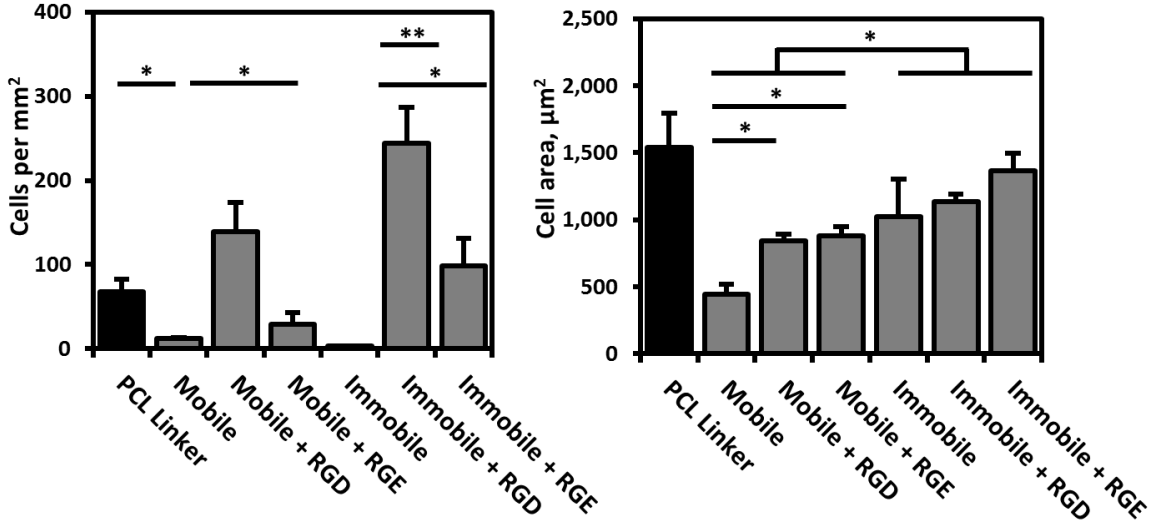

d)

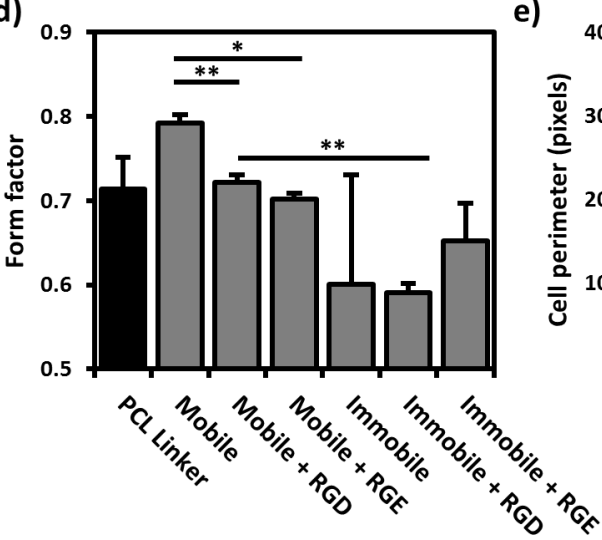

e)
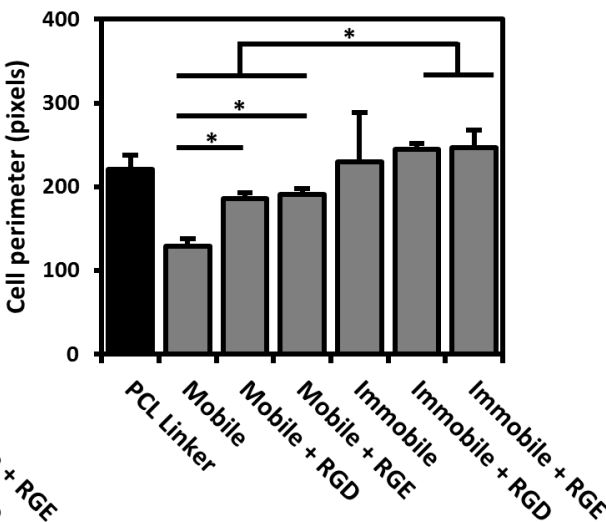

Figure 6.6: iMSC initial cell adhesion on BSLBs; PCL samples modified with liquid- and gel-state BSLBs for the mobile or immobile presentation of RGD or control RGE palmitoylated peptides. Samples were stained with DAPI (Blue) and phalloidin-AF488 (Green) for nucleus and cytoskeleton, respectively. a) Fluorescent micrographs of iMSCs cultured for 2 hours on BSLBs (inset shows low 
magnification overview) and quantification of b) cells per $\mathrm{mm}^{2}$, c) cell area, d) form factor $\left(4^{*} \pi^{*}\right.$ cell area/perimeter ${ }^{2}$ ) and e) cell perimeter. Data presented as mean \pm SD, and compared using 1-way Anova, $* p<0.05 * *$ and $p<0.01$. Scale bar $200 \mu \mathrm{m}$.

A recent study confirmed the absence of traction forces when cells were cultured on mobile SLBs decorated with RGD peptides. ${ }^{29}$ With respect to MSCs, it has been suggested that cell-generated traction forces are heavily involved in detecting biophysical cues and in inducing subsequent differentiation pathways. ${ }^{30}$ To find whether differences in lateral mobility between mobile and immobile RGD-modified BSLBs would translate into preferential differentiation towards a certain cell lineage, iMSC differentiation was investigated (Figure 6.7). To this end, the iMSC cell culture media was exchanged after 2 hours for a double-induction media for adipo- and osteogenesis. ${ }^{5}$ After 1 week of iMSC culture under double-induction conditions, surface mitigated preferential differentiation was observed. To gain insights into the integrity of the BSLB layer during the entire cell culture period, the FRAP properties of a dye labelled SLB on a glass surface (Figure S8) were studied, while direct observation using fluorescence microscopy of the BSLB on PCL during cell culture was inaccessible due to the opaqueness of PCL sheets. From the fluorescence recovery $D$-values were estimated over the course of 1 week and remained similar for 3 days of cell culture in fully supplement cell culture media. Beyond this day the BSLB seems to have lost much of its integrity, nonetheless and much to our surprise, mobile BSLBs that display palmitoylated RGD peptides significantly drive iMSCs towards the adipogenic lineage as indicated by elevated Oil Red $O$ levels, whereas on the immobile BSLB showed differentiation into osteogenic lineage as indicated by elevated alkaline phosphatase levels. In both cases, a ca. two-fold increase in induction was observed when compared to the underlying cholesterol-modified PCL (Figure 6.7a). A qualitative assessment by means of histological stainings supports our findings (Figure 6.8b). The remarkable observation of BSLB surface mitigated preferential differentiation is in agreement with reports that show promoted osteogenesis occurring on stiff surfaces, while up-regulation of the adipogenic marker Oil Red $\mathrm{O}$ can occur on soft substrates due to decreased traction force generation. ${ }^{3,5,30,31}$ A recent study proposed that MSCs assemble their fibronectin environment within the first 24 hours and encode their physical environment by a strain of fibronectin ECM fibrils, which they self-adjust by their own traction forces, thereby highlighting the importance of guiding the initial cell-interface interaction. ${ }^{32}$ Our findings suggest a strong effect of ligand mobility in cell physiology and that the integrity of the BSLB is sufficiently long for this guiding process. Groves and coworkers also showed a strong involvement of the gel state and reported that the EphA2 receptor, a part of juxtacrine signalling, and spatial organization is strongly dependent on the gel state of the SLB onto which cells are grown that express the EphA2 receptor tyrosine kinase. ${ }^{33}$ The findings presented in this study support the proposition that the 
mechanical and biological properties of the underlying BSLB support is less involved in cell fate decision then in traditional material coatings.

a)

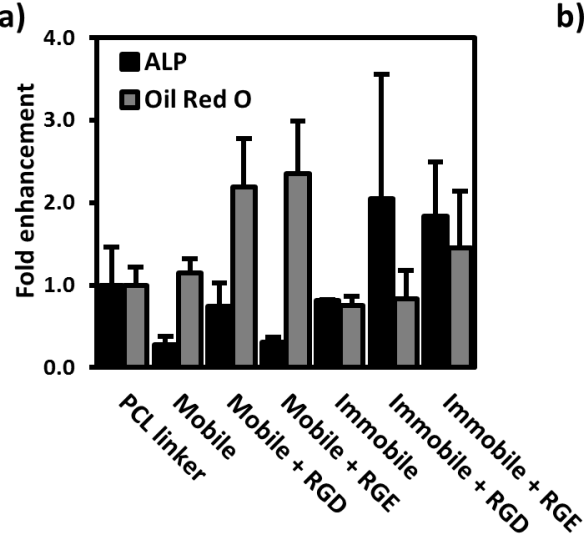

b)

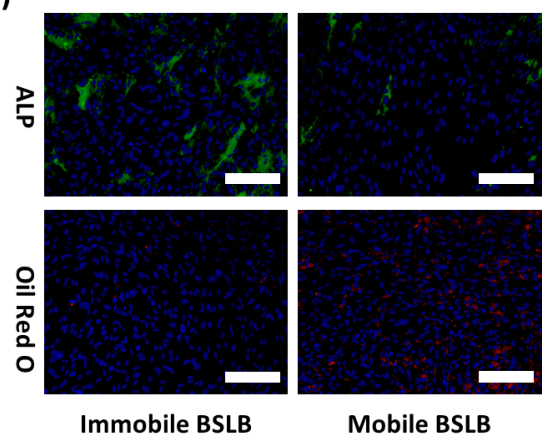

Figure 6.7: Effect of RGD lateral mobility on MSC differention on BSLBs; hMSC cell culture after 1 week in double induction media i.e. osteo- and adipogenic. a) Quantified Oil Red $\mathrm{O}$ and ALP in cell lysates. Data presented as mean $\pm S D(n>3)$. 2-way Anova showed significant differences between mobile and immobile BSLB, $p<0.05$. b) Example of a processed histological staining of ALP and Oil Red $O$ on mobile and immobile BSLBs. Nuclei (DAPI, Blue) ALP (Green) and Oil Red O (Red), scale bar $200 \mu \mathrm{m}$.

\subsection{Conclusions}

Our membrane mimetic coating strategy provides a versatile means to fine-tune the cellular interface on surfaces of any biopolymer. Besides the ease with which the ligand density and the type of ligand can be altered, traction forces as determined by ligand lateral mobility can be manipulated. The latter has been shown to influence stem cell behaviour similar that was observed previously by Disscher and Huck and co-workers upon varying substrate stiffness and ECM protein flexibility, respectively. Moreover, the non-fouling nature of the BSLB coating and the use of known, highly biocompatible lipids prevents undesirable cellular responses from occurring. The use of biomimetic SLB coatings for biomaterials suggests both full mechanical and full biological decoupling of bulk material properties from cell fate. The proposed surface modification strategy can be executed on virtually any polymeric support that is currently applied in the clinic and therefor our coating strategy is of paramount biomedical importance as universal coating strategy prevents individual chemical tailoring of each and every polymer surface. 


\subsection{Acknowledgements}

Dr. C. Nicosia is gratefully acknowledged for his help with the synthesis of the cholesterol linker. Prof. Dr. H. L. Offerhaus and Dr. A. L. Fussell are gratefully acknowledged for their help with CARS and two-photon microscopy.

\subsection{Experimental section}

\subsubsection{Materials}

All starting materials and chemicals were purchased from Sigma-Aldrich, Fluka, Serva, Avanti Polar Lipids, Invitrogen, ABCR, Ssens, Gibco, Lonza, Roche and they were used as received, unless otherwise stated. MilliQ water with a resistivity higher than $18 \mathrm{M} \Omega \mathrm{cm}^{-1}$ was used in all experiments.

Cholesterol linker. Synthesizes was based on literature and used with modification, see Figure 59. ${ }^{34}$ In short, to a stirred solution of 1.0 equivalents $i$ (n-Boc- 2,2'[ethylenedioxy]diethylamine, Sigma-Aldrich) and 1.1 equivalents ii (cholesteryl chloroformate, Sigma-Aldrich) in anhydrous toluene $(25 \mathrm{~mL}), 1.5$ equivalents of DIPEA (diisopropylethylamine, Sigma-Aldrich) and a catalytic amount of DMAP (4Dimethylaminopyridine, Sigma-Aldrich) were added. The reaction mixture ( 15 mmol scale) was refluxed under argon atmosphere for 17 hours. Subsequently, the solvent was removed under reduced pressure to obtain an oily residue. Flash column chromatography was performed using $40 \%$ ethyl acetate $+60 \%$ hexane as eluent $\left(R_{f}=0.22\right)$. The solvent was removed under reduced pressure and the product iii was found to be an off white sticky residue. Product iii was dissolved in DCM (dichloromethane) and added in a dropwise manner to a stirred solution of 4 equivalents of TFA (trifluoroacetic acid) in DCM. Deprotection was left for 23 hours at room temperature (RT) under continuous stirring. Deprotection was monitored by TLC. Solvent was removed under reduced pressure and the residue was dissolved in DCM and neutralized with TEA (triethylamine). Flash column chromatography was performed using $1 \% \mathrm{NH}_{3}$ (aq) solution ( $25 \% \mathrm{NH}_{3}$ in water), $9 \% \mathrm{MeOH}$ and $90 \%$ chloroform $\left(R_{f, \text { frac13-16 }}=0.38, R_{f, \text { frac17-35 }}=0.24\right)$. After solvent removal a yellowish sticky residue was obtained ( $76 \%$ yield). The purified product was stored at $-20^{\circ} \mathrm{C}$ until further use and will be referred to as 'cholesterol linker'. See Figure S10 for ESI-ToF (Waters) and ${ }^{1} \mathrm{H}$ NMR (Varian Unity INOVA, $300 \mathrm{MHz}$ ) analysis.

Palmitoylated RGD. Palmitoylated peptides were synthesized as described in Chapter 5. 


\subsubsection{Methods}

PCL sample preparation. (Bio) polymer samples were prepared by means of spin-coating or solvent-casting from chloroform. Spin-coated PCL samples were prepared on either glass substrates (Menzel-gläser) or gold-coated glass disks (20 nm Au on glass, Ssens). A 0.25 wt\% PCL (Mn 45 kDa, Sigma-Aldrich) chloroform solution was used for spin-coating the various substrates. Here, an initial ramp at $500 \mathrm{rpm}$ for 3 seconds was followed by a 30 seconds ramp at 4,000 rpm.

In addition to spin-coated samples, solvent-cast PCL films were prepared. ${ }^{35}$ In short, granules ( 2 grams) of the polymer were dissolved in $40 \mathrm{~mL}$ chloroform and cast into a large glass petri dish $(\varnothing 14.5 \mathrm{~mm}$ ) that was silanized with a PFDTS anti-sticking layer $(1 \mathrm{H}, 1 \mathrm{H}, 2 \mathrm{H}, 2 \mathrm{H}$-Perfluorodecyltrichlorosilane, $\geq 97 \%, \mathrm{ABCR} \mathrm{GmbH})$ deposited from the gas hase $\mathrm{o} / \mathrm{n}$. Here, $50 \mu \mathrm{L}$ of PFDTS and the petri dish were placed in a desiccator and pumped down for 10 minutes. The PFDTS modified petri dish was washed with acetone before use. After adding the dissolved PCL polymer in chloroform, the petri dish lid was placed back allowing for slow evaporation of the solvent ( $\sim 8$ hours). After the solvent had evaporated, the samples were annealed above the melting temperature to yield a smooth polymer surface at the glass interface. All of the subsequent modifications and analysis were performed on the glass side of PCL solvent-cast films.

Surface activation. The plasma treatment step was performed using 40 watts of power at 200 mtorr of vacuum pressure (Plasma Prep II, SPI supplies). Care was taken to use the samples immediately after OP treatment.

Cholesterol linker coupling. OP treated PCL samples were incubated in a $1 \mathrm{mM}$ ethanolic

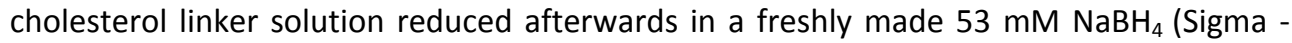
Aldrich) solution. Until use the samples were stored at room temperature under nitrogen atmosphere.

PCL surface characterization. The PCL samples were studied using a wide range of surface analysis tools. Water contact angle (Krüss), X-ray photoelectron spectroscopy (Quantera SXM scanning XPS microprobe, Physical Electronics), IR spectroscopy (ATR and PM-IRRAS, Thermo Scientific), Atomic force microscopy (Ntegra Spectra, NT-MDT). CARS and Twophoton experiments were conducted on a 'In-house-build' set-up courtesy of the Optical sciences group University of Twente

Vesicle preparation. 1,2-Dioleoyl-sn-glycero-3-phosphocholine (DOPC, Avanti Polar Lipids) was stored as a $25 \mathrm{mg} / \mathrm{mL}$ stock solution in chloroform at $-20^{\circ} \mathrm{C}$. 1,2-Dipalmitoyl-sn- 
glycero-3-phosphocholine (DPPC, Avanti Polar Lipids) was stored as a $25 \mathrm{mg} / \mathrm{mL}$ stock solution in chloroform at $-20^{\circ} \mathrm{C}$. For fluorescent of the lipid bilayers either Oregon Green or Texas Red-1,2-dihexadecanoyl-sn-glycero-3-phosphoethanolamine, (OG-DHPE or TRDHPE, Invitrogen), $1 \mathrm{mg} / \mathrm{mL}$ stock solutions in methanol at $-20^{\circ} \mathrm{C}$, were used. Desired molar ratios of lipids and palmitoylated peptides were mixed and dried under a flow of nitrogen in a glass vial, and subsequently placed under vacuum for at least 1 hour. The resulting lipid film was re-suspended by vortexing in MilliQ water to form multilamellar vesicles (MLVs) at $1 \mathrm{mg} / \mathrm{mL}$ at $60^{\circ} \mathrm{C}$. The MLV solution was extruded 11 times through a $100 \mathrm{~nm}$ polycarbonate membrane (Avanti Polar Lipids) at $60^{\circ} \mathrm{C}$. The resulting large unilaminar vesicles (LUVs) were characterized by dynamic light scattering (DLS, Nanotrac, Microtrac) and stored until use at $4^{\circ} \mathrm{C}$ for a maximum of two weeks.

BSLB formation. Biomaterial supported lipid bilayer (BSLB) formation was achieved by dilution of the LUV solution to $0.5 \mathrm{mg} / \mathrm{mL}$ in phosphate-buffered saline $(0.01 \mathrm{M}$ PBS, Gibco, lacking $\mathrm{MgCl}_{2}$ and $\mathrm{CaCl}_{2}$ ). Prior to LUV incubation, the $\mathrm{PCL}$ samples (Greiner) were flushed briefly with $0.01 \mathrm{M}$ PBS. Afterwards the samples were incubated with the vesicle suspension for at least 30 minutes to allow for vesicle adsorption and rupture to occur. In addition, the samples were frozen to ensure maximum vesicle rupture. ${ }^{23}$ Afterwards the samples were washed with DI. For cell culture experiments, the samples were incubated overnight with $0.01 \mathrm{M}$ PBS containing $100 \mathrm{U} / \mathrm{mL}$ Pen/Strep (Gibco). Solution exchange occurred via serial dilution to minimize dehydration damage.

Protein fouling. Fluorescently labelled BSA was prepared and used to quantify amount of protein adsorbed to the sample surface. ${ }^{15}$ Briefly, NHS activated Dylight-488 was incubated with BSA and purified using spin columns following the protocol provided by the manufacturer (Thermo Scientific). The BSLB modified samples were incubated with 50 $\mu \mathrm{g} / \mathrm{mL}$ BSA conjugates in PBS for 20 minutes. Subsequent washings using PBS ensured loosely bound protein to be removed. Adsorbed protein was desorbed in SDS solution for 1 hour at r.t. and quantified using a plate reader (Victor, Perkin-Elmer) and a standard curve. In the case of the dehydrated BSLB the buffer was removed and the surface was exposed to air. Subsequently, PBS buffer was added to rehydrate the BSLB samples.

Cell culture sample preparation. Circular polymer samples were punched out of a cholesterol linker modified PCL polymer sheets (as described before) of $6 \mathrm{~mm}$ in diameter. These samples were placed in a 96 well plate and fixed using cut Eppendorf P1000 tips, see Figure S11. Solution exchange occurred via serial dilution to minimize BLSB damage.

Cell culture. For iMSC proliferation, cells were cultured to $~ 90 \%$ confluence in basal media [ $\alpha$-MEM (Gibco), 10\% FBS (Lonza), 2 mM L-glutamin (Gibco), 0.2 mM ascorbic acid (Sigma- 
Aldrich), 100U/mL Pen/Strep (Gibco)] supplemented with $1 \mathrm{ng} / \mathrm{mL}$ bFGF (Instruchemie). Double induction media consisted of one part osteogenic media [ $\alpha$-MEM (Gibco), 10\% FBS (Lonza), $2 \mathrm{mM}$ L-glutamin (Gibco), $0.2 \mathrm{mM}$ ascorbic acid (Sigma-Aldrich), Pen/Strep (Gibco), $10^{-8} \mathrm{M}$ dexamethasone (Sigma-Aldrich)] and one part adipogenic media [DMEM (Gibco), $100 \mathrm{U} / \mathrm{mL}$ Pen/Strep (Gibco), 10\% FBS (Lonza), $0.2 \mathrm{mM}$ Indomethacin (SigmaAldrich), $10^{-6} \mathrm{M}$ dexamethasone (Sigma-Aldrich), $10 \mathrm{mg} / \mathrm{mL}$ Human insulin (SigmaAldrich)]. Cells were seeded at a density of $2,500 \mathrm{cell} / \mathrm{cm}^{2}$ and for the differentiation experiments at 25,000 cells $/ \mathrm{cm}^{2}$.

Cell analysis. For fluorescent labelling cell were fixed for 15 minutes in $10 \%$ formalin solution (Sigma-Aldrich) and permeabilized for 4 minutes using PBS-0.1\% Triton X100. DAPI (Invitrogen) and Phalloidin-alexa488 (Invitrogen) were used to visualize the nuclei and actin cytoskeleton, respectively. Phalloidin-alexa488 was left to incubate for 20 minutes at room temperature. in the dark and counterstained with DAPI (1:100 in PBS) is added and left to incubate for 10 minutes. After each step in the mentioned procedure the cells were washed with PBS. Due to the crystalline nature of PCL, samples were carefully removed from the well plate and imaged upside down.

ALP and Oil Red $\mathbf{O}$ quantification. ALP was quantified using CDPstar (Roche). CyQuant (Invitrogen) was used according to manufactures instructions to determine DNA content to correct for cell count. Part of the cell lysate was added to CDPstar and left in the dark for 30 minutes at room temperature. CyQuant fluorescence was measured at emission $500 \mathrm{~nm} /$ excitation $480 \mathrm{~nm}$ and CDPstar luminescence was measured (Victor, PerkinElmer). In the case of Oil Red O, cells were fixed in 10\% formalin (Sigma-Aldrich) for 15 minutes. Samples were washed in $\mathrm{dH}_{2} \mathrm{O}$ and incubated for 5 minutes in $60 \%$ isopropanol in $\mathrm{dH}_{2} \mathrm{O}$. Subsequently, filtered Oil Red O solution (Sigma-Aldrich, stock $500 \mathrm{mg}$ in $99 \mathrm{~mL}$

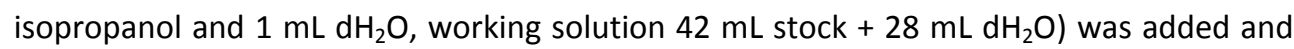
left to incubate for 5 minutes. After rinsing the samples extensively with $\mathrm{dH}_{2} \mathrm{O}$, Oil Red $\mathrm{O}$ was quantified by lysing the cells in 4\% Ipagal (Sigma-Aldrich) in isopropanol for 15 minutes while shaking. Absorbance was measured using a microplate reader at $540 \mathrm{~nm}$ (Bio-TEK Instruments).

Histological staining. For Oil Red $\mathrm{O}$ staining, cells were fixed in $10 \%$ formalin (SigmaAldrich) for 15 minutes. Samples were washed in DI and incubated for 5 minutes in $60 \%$ isopropanol in DI. Subsequently, filtered Oil Red O solution (Sigma-Aldrich, stock $500 \mathrm{mg}$ in $99 \mathrm{~mL}$ isopropanol and $1 \mathrm{~mL} \mathrm{DI}$, working solution $42 \mathrm{~mL}$ stock $+28 \mathrm{~mL}$ DI) was added and left to incubate for 5 minutes. Afterwards cells were rinsed extensively with DI. For ALP staining, cells were fixed for 1 hour in $10 \%$ formalin and stained using the Alkaline Phosphatase kit (Sigma-Aldrich) according to manufactures instructions. Oil Red O and ALP 
were imaged with bright field microscopy. In both cases, DAPI was used to counterstain the cell nucleus. Image analysis with ImageJ and CellProfiler allowed us to extract the ALP and Oil Red O signal reliably in some cases.

Fluorescence microscopy. An Olympus inverted IX71 epi-fluorescence research microscope with a Xenon X-cite 120PC as light source and a digital Olympus DR70 camera for image acquisition was used to acquire fluorescence micrographs at $10 x$ and $20 x$ magnification. DAPI was imaged using $325 \leq \lambda_{\mathrm{ex}} \leq 375 \mathrm{~nm}$ and $\lambda_{\mathrm{em}}>420 \mathrm{~nm}$; OG-DHPE Phalloidin-alexa488 were imaged using $460 \leq \lambda_{\mathrm{ex}} \leq 490 \mathrm{~nm}$ and $\lambda_{\mathrm{em}}>525 \mathrm{~nm}$; TR-DHPE was imaged using $510 \leq \lambda_{\mathrm{ex}} \leq 550 \mathrm{~nm}$ and $\lambda_{\mathrm{em}}>590 \mathrm{~nm}$. Confocal scanning laser microscopy images were acquired with a Nikon A1. FRAP data was obtained using a 20x objective.

Data analysis. Image analysis was performed using ImageJ (NIH), Origin (OriginLab) and Excel (Microsoft). Cell shape analysis was performed with CellProfiler ${ }^{36}$. The diffusion coefficient was deduced using modified Bessel functions as described by Soumpasis et al. 1983. ${ }^{24}$ Data was corrected for bleaching during image acquisition and normalized and processed with FRAPAnalyser (University of Luxembourg).

Statistics. Student T-test and Anova were used for hypotheses testing. Prior to Anova evaluation normal distribution of the data sets was confirmed by performing the Shaphiro-Wilk test. Grubb's test was used to remove potential outliers and a Post-hoc Tukey's test provided means comparison. Equal variance was confirmed by Levene's test. Excel (Microsoft), SPSS (IBM) and Origin (OriginLab) were used for statistical analysis 


\subsection{References}

Stevens, M. M.; George, J. H. Science 2005, 310, 1135-1138.

Mager, M. D.; LaPointe, V.; Stevens, M. M. Nat. Chem. 2011, 3, 582-589.

Watt, F. M.; Huck, W. T. S. Nat. Rev. Mol. Cell Biol. 2013, 14, 467-473.

Lutolf, M. P.; Hubbell, J. A. Nat. Biotechnol. 2005, 23, 47-55.

Trappmann, B.; Gautrot, J. E.; Connelly, J. T.; Strange, D. G. T.; Li, Y.; Oyen, M. L.; Cohen Stuart, M. A.; Boehm, H.; Li, B.; Vogel, V.; Spatz, J. P.; Watt, F. M.; Huck, W. T. S. Nat. Mater. 2012, 11, 642-649.

MacQueen, L.; Sun, Y.; Simmons, C. A. J. R. Soc. Interface 2013, 10.

Engler, A. J.; Sen, S.; Sweeney, H. L.; Discher, D. E. Cell 2006, 126, 677-689.

Frith, J. E.; Mills, R. J.; Cooper-White, J. J. J. Cell Sci. 2012, 125, 317-327.

Hench, L. L.; Polak, J. M. Science 2002, 295, 1014-+.

Place, E. S.; Evans, N. D.; Stevens, M. M. Nat. Mater. 2009, 8, 457-470.

Chaudhuri, O.; Mooney, D. J. Nat. Mater. 2012, 11, 568-569.

Bellis, S. L. Biomaterials 2011, 32, 4205-4210.

Ratner, B. D.; Bryant, S. J. Annu. Rev. Biomed. Eng. 2004, 6, 41-75.

Riedel, T.; Riedelová-Reicheltová, Z.; Májek, P.; Rodriguez-Emmenegger, C.; Houska, M.; Dyr, J. E.; Brynda, E. Langmuir 2013, 29, 3388-3397.

Grafahrend, D.; Heffels, K. H.; Beer, M. V.; Gasteier, P.; Moller, M.; Boehm, G.; Dalton, P. D.; Groll, J. Nat. Mater. 2011, 10, 67-73.

Deng, Y.; Wang, Y.; Holtz, B.; Li, J.; Traaseth, N.; Veglia, G.; Stottrup, B. J.; Elde, R.; Pei, D.; Guo, A.; Zhu, X. Y. J. Am. Chem. Soc. 2008, 130, 6267-6271.

Xue, C. Y.; Yang, K. L. Langmuir 2007, 23, 5831-5835.

Zander, N. E.; Orlicki, J. A.; Rawlett, A. M.; Beebe, T. P., Jr. Biointerphases 2010, 5, 149-158.

Calabretta, A.; Wasserberg, D.; Posthuma-Trumpie, G. A.; Subramaniam, V.; van Amerongen, A.; Corradini, R.; Tedeschi, T.; Sforza, S.; Reinhoudt, D. N.; Marchelli, R.; Huskens, J.; Jonkheijm, P. Langmuir 2011, 27, 1536-1542.

Krabbenborg, S. O.; Nicosia, C.; Chen, P. K.; Huskens, J. Nat. Commun. 2013, 4.

Knoll, W. Handbook of biofunctional surfaces, 2013.

Attwood, S. J.; Choi, Y.; Leonenko, Z. Int. J. Mol. Sci. 2013, 14, 3514-3539.

Sugihara, K.; Jang, B.; Schneider, M.; Voros, J.; Zambelli, T. Soft Matter 2012, 8, 5525-5531.

Soumpasis, D. M. Biophys. J. 1983, 41, 95-97.

Andreasson-Ochsner, M.; Romano, G.; Hakanson, M.; Smith, M. L.; Leckband, D. E.; Textor, M.; Reimhult, E. Lab Chip 2011, 11, 2876-2883.

Ruoslahti, E. Annu. Rev. Cell Dev. Biol. 1996, 12, 697-715.

Prockop, D. J. Science 1997, 276, 71-74.

Simonsen, J. L.; Rosada, C.; Serakinci, N.; Justesen, J.; Stenderup, K.; Rattan, S. I.; Jensen, T. G.; Kassem, M. Nat. Biotechnol. 2002, 20, 592-596.

Yu, C.-h.; Rafiq, Nisha Bte M.; Krishnasamy, A.; Hartman, Kevin L.; Jones, Gareth E.; Bershadsky, Alexander D.; Sheetz, Michael P. Cell Reports 2013, 5, 1456-1468.

Vincent, L. G.; Engler, A. J. Nat. Mater. 2013, 12, 384-386.

Khetan, S.; Guvendiren, M.; Legant, W. R.; Cohen, D. M.; Chen, C. S.; Burdick, J. A. Nat. Mater. 2013, 12, 458-465.

Li, B. J.; Moshfegh, C.; Lin, Z.; Albuschies, J.; Vogel, V. Sci. Rep. 2013, 3.

Salaita, K.; Nair, P. M.; Petit, R. S.; Neve, R. M.; Das, D.; Gray, J. W.; Groves, J. T. Science 2010, 327, 13801385.

Han, X.; Achalkumar, A. S.; Cheetham, M. R.; Connell, S. D. A.; Johnson, B. R. G.; Bushby, R. J.; Evans, S. D. ChemPhysChem 2010, 11, 569-574.

Tang, Z. G.; Black, R. A.; Curran, J. M.; Hunt, J. A.; Rhodes, N. P.; Williams, D. F. Biomaterials 2004, 25, 4741-4748.

Carpenter, A. E.; Jones, T. R.; Lamprecht, M. R.; Clarke, C.; Kang, I. H.; Friman, O.; Guertin, D. A.; Chang, J. H.; Lindquist, R. A.; Moffat, J.; Golland, P.; Sabatini, D. M. Genome Biol. 2006, 7. 


\subsection{Supporting information}

a)

c)

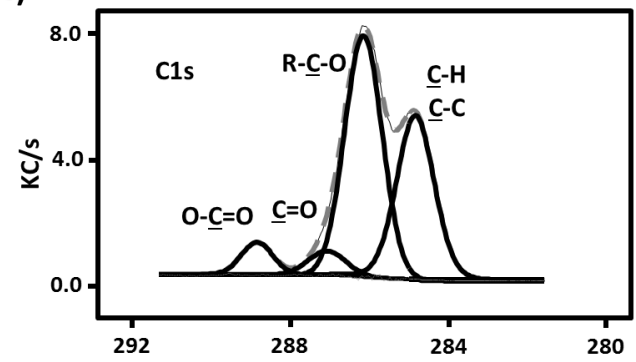

d)

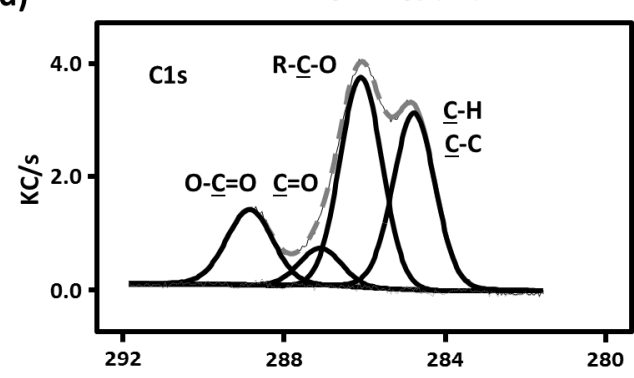

e)

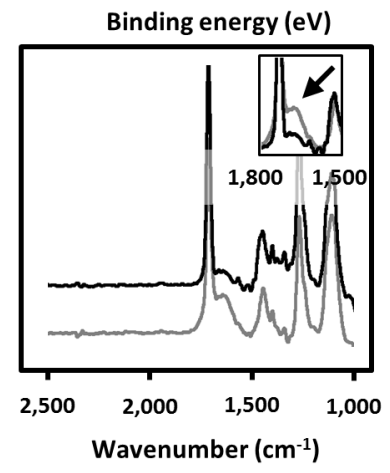

b)
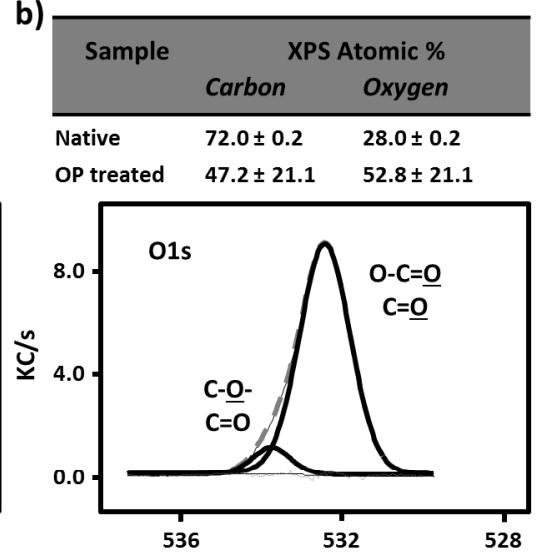

Binding energy (eV)

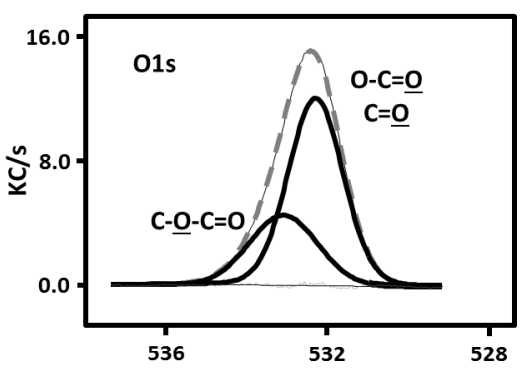

f)

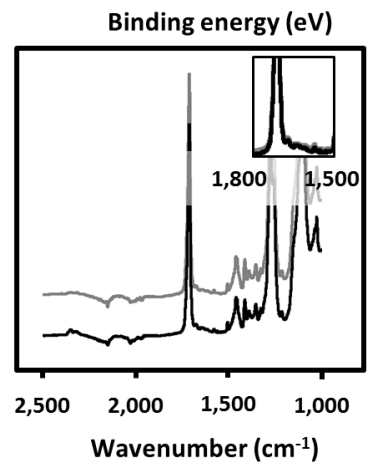

Figure S1: Generating aldehyde groups on PEOT-PBT by an OP treatment; a) results of Purpald reaction to stain aldehydes at the surface of the OP treated polymer samples. The atomic composition given in $\mathbf{b})$ was determined by XPS, mean \pm SD $(n=5)$. XPS spectra of $\mathbf{c}) \mathrm{C} 1 \mathrm{~s}$ and $01 \mathrm{~s}$ of the native PEOT-PBT and d) OP treated PEOT-PBT. IR-spectra obtained with e) PM-IRRAS and f) FTIRATR of native (Black) and OP treated (Grey) PEOT-PBT samples. Arrow indicates shoulder in the carbonyl region. 
a)

c)

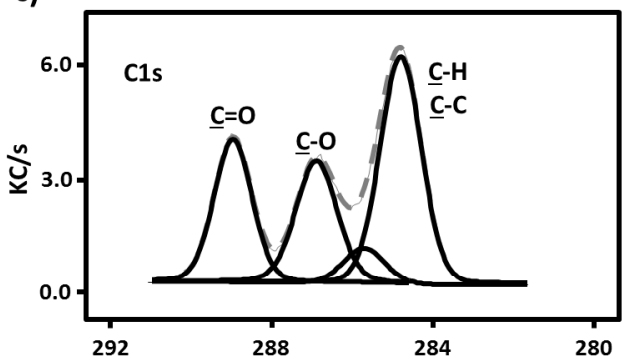

d)

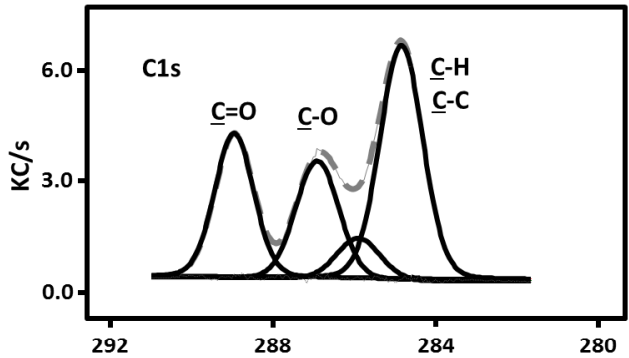

e)

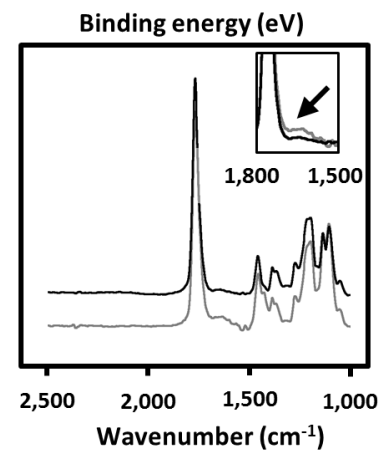

b)
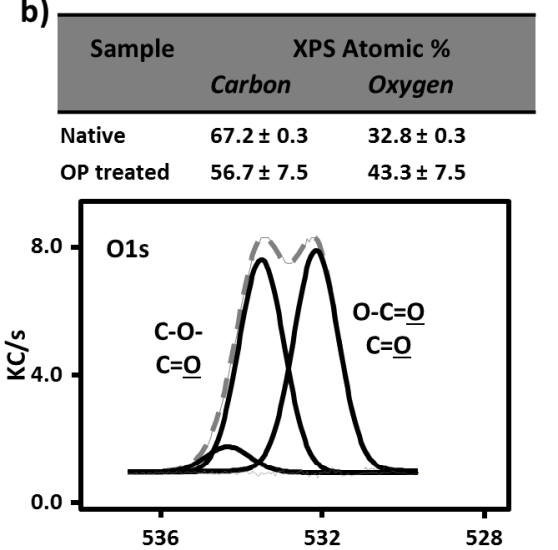

Binding energy (eV)

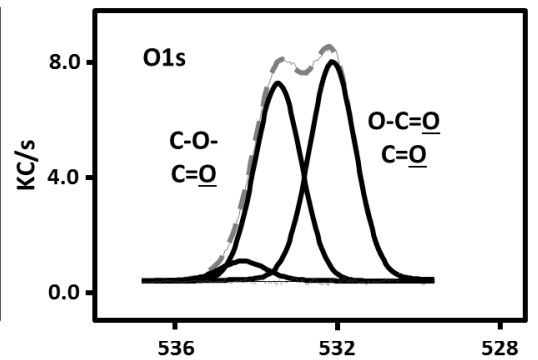

f)

Binding energy (eV)

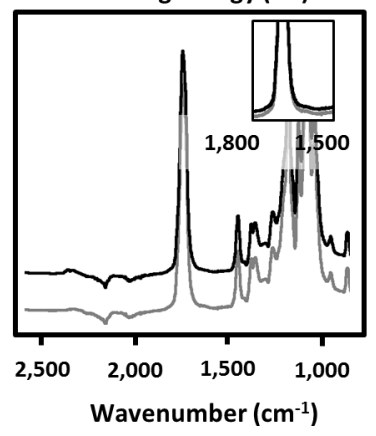

Figure S2: Generating aldehyde groups on PLA-PCL by an OP treatment; a) results of Purpald reaction to stain aldehydes at the surface of the OP treated polymer samples. The atomic composition given in b) was determined by XPS, mean \pm SD $(n=5)$. XPS spectra of $\mathbf{c})$ C1s and O1s of the native PLA-PCL and d) OP treated PLA-PCL. IR-spectra obtained with e) PM-IRRAS and f) FTIR-ATR of native (Black) and OP treated (Grey) PLA-PCL samples. Arrow indicates shoulder in the carbonyl region. 
a)

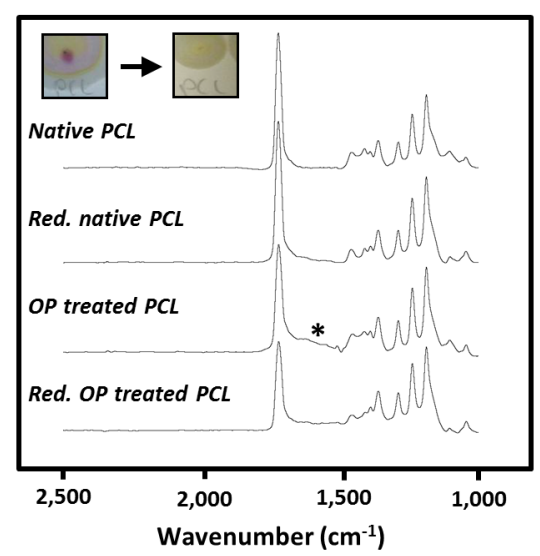

b)

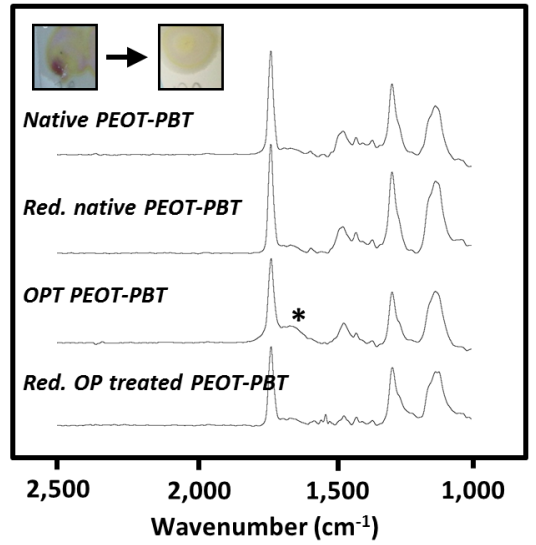

c)

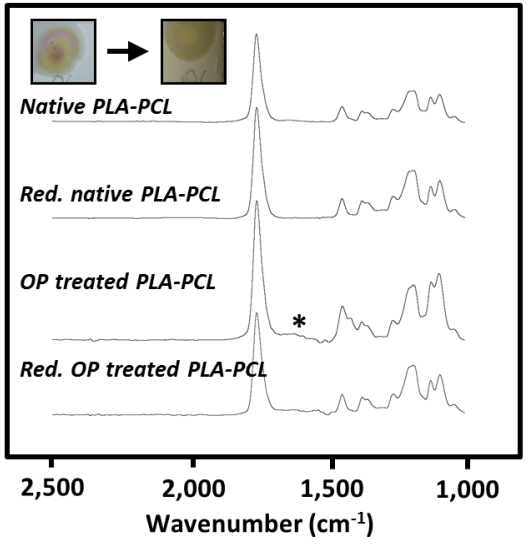

Figure S3: The effect of a reduction step on native and OP treated biopolymers; OP treated and untreated samples were analysed using PM-IRRAS before and after reduction in $\mathrm{NaBH}_{4}$. Spin-coated samples on $20 \mathrm{~nm}$ gold discs were measured. After OP treatment and reduction the shoulder (asterix) near the carbonyl band decreased. Insets show the corresponding Purpald staining of OP treated polymers before and after reduction. 

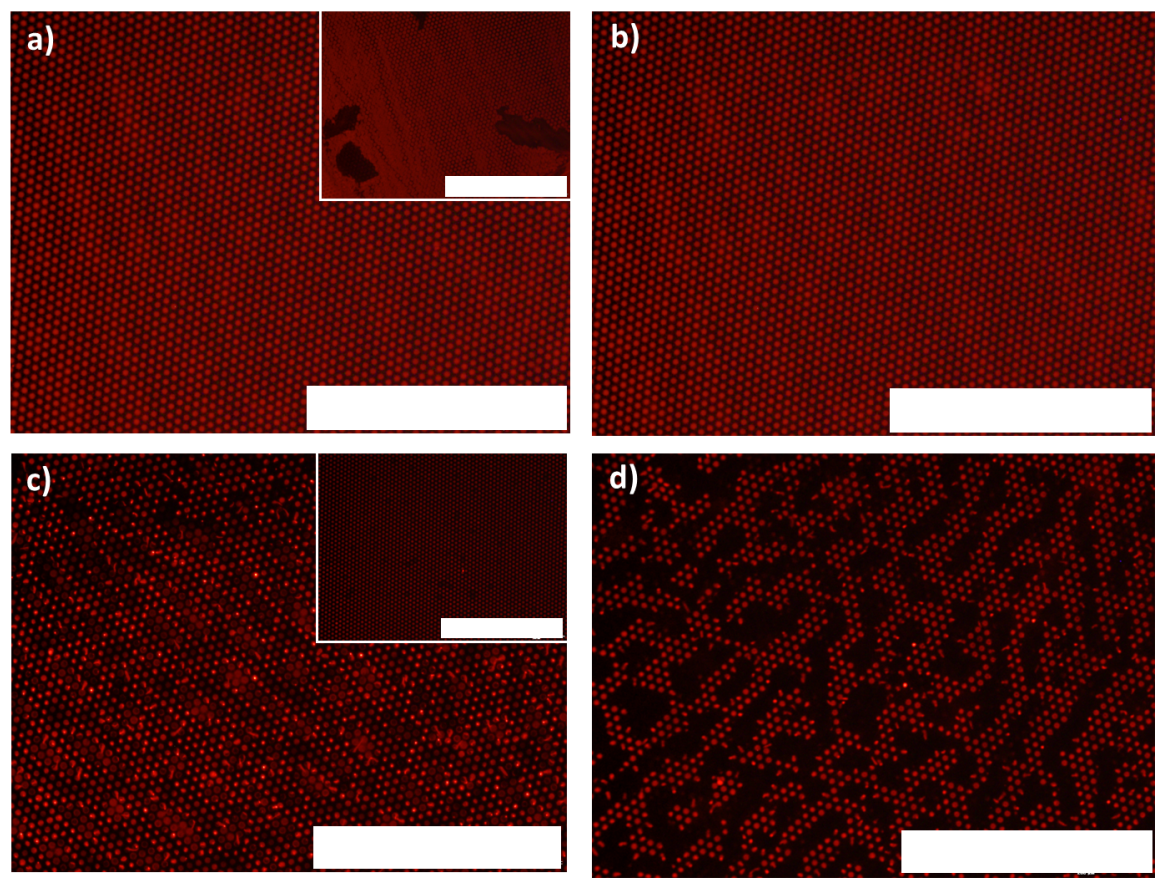

Figure S4: Micro-contact printing of rhodamine $B$ ethylenediamine on OP treated PEOT-PBT and PLA-PCL samples; OP treated PEOT-PBT samples a) directly after printing or b) following an extensive washing step in methanol and OP treated PLA-PCL samples c) directly after printing or d) following an extensive washing step in methanol. Insets shows non-OP treated polymers directly after printing. In this case, no patterns were visible after washing. Scale bar $400 \mu \mathrm{m}$. 


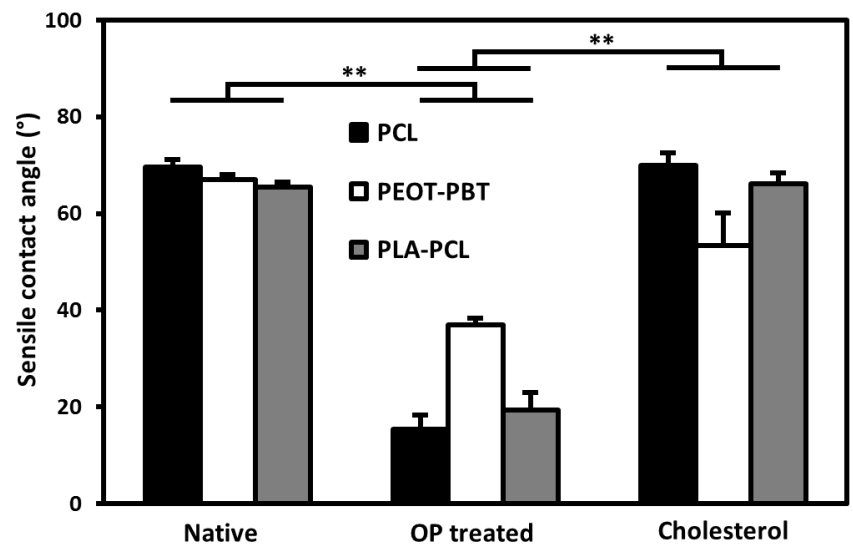

Figure S5 Coupling of cholesterol linker to OP treated polymers; sensile water contact angle of PCL, PEOT-PBT and PLA-PCL polymers treated with OP and incubated for 1 hour with the cholesterol linker Data presented as mean \pm SD $(n=3)$, and compared using Student T-test, ${ }^{* *}$ and $p<0.01$.

a)

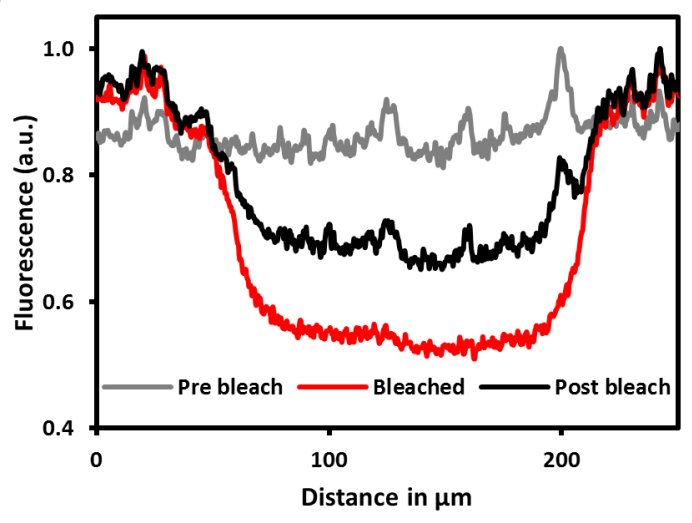

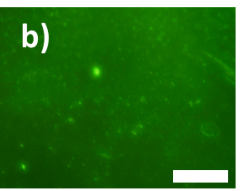

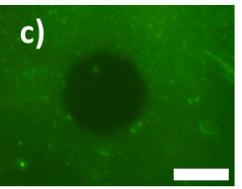

d)

Figure S6: Air-stability of BSLB on PCL; epi-fluorescent analysis of a liquid state bilayer doped with 0.2 mol\% OG-DHPE applied on a 2D cholesterol-modified PCL film. a) Profile plots of the BSLB after air exposure, bleaching and recovery. Corresponding epi-fluorescent images of $\mathbf{b}$ ) the pre bleach, $\mathbf{c}$ ) post bleach and d) after several minutes of recovery. Scale bar $100 \mu \mathrm{m}$. 


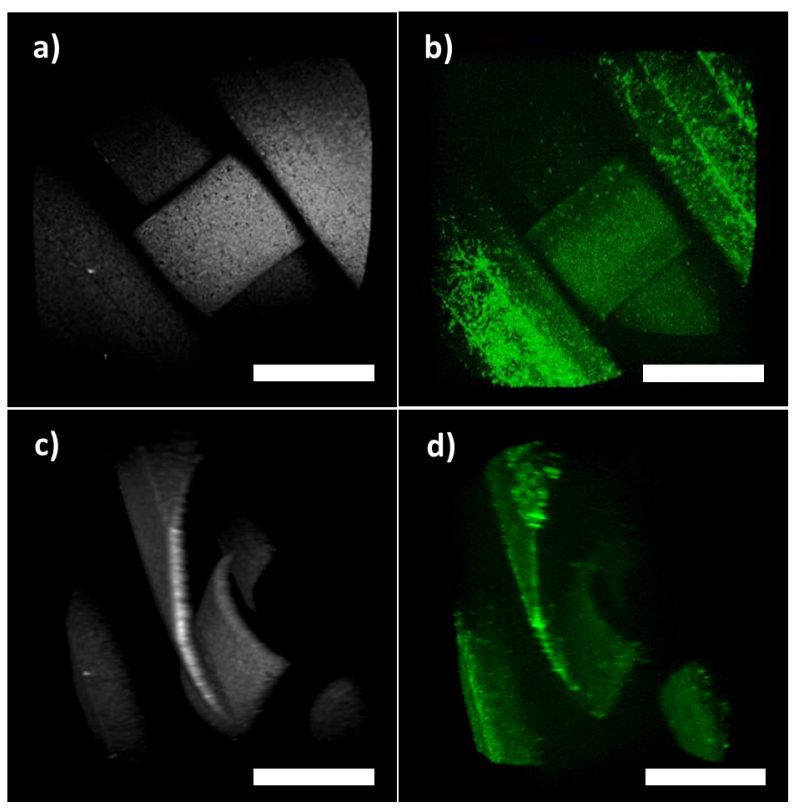

Figure S7: CARS and two-photon fluorescence; CARS and two-photon analysis of a mobile BSLB doped with 0.2 mol\% OG-DHPE on a 3D PCL fibre mesh. a) Stacked z-slices of the 2,920 $\mathrm{cm}^{-1}$ CARS signal of the PCL polymer (C-H stretching) and $\mathbf{b}$ ) the two-photon fluorescence of OG-DHPE, $\mathbf{c}$ ) and d) show angled view, scale bar $500 \mu \mathrm{m}$.

a)

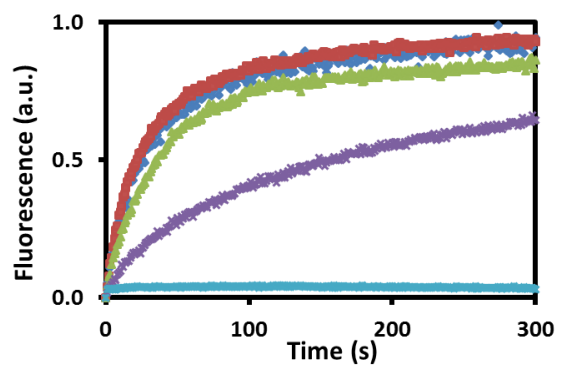

c) b)

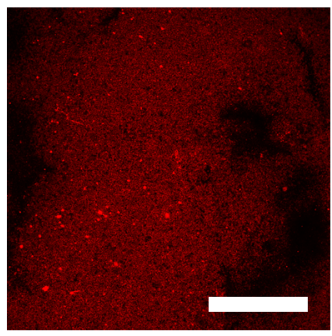

\begin{tabular}{lccccc}
\hline & Day 0 & Day 2 & Day 3 & Day 4 & Day 7 \\
\hline $\boldsymbol{D}\left(\boldsymbol{\mu \mathrm { m } ^ { 2 } / \mathrm { s } )}\right.$ & $1.23 \pm 0.12$ & $0.68 \pm 0.25$ & $0.50 \pm 0.11$ & $0.18 \pm 0.01$ & - \\
Mob. Frac. (\%) & $96.94 \pm 0.36$ & $93.92 \pm 2.62$ & $90.24 \pm 1.31$ & $71.45 \pm 1.31$ & -
\end{tabular}

Figure S8: Analysis of SLB stability in vitro on glass substrates; a) FRAP recovery curves and $D$-values obtained at different intervals in the presence of cells. Note bleach spot was not equal for the plots that are shown. b) Fluorescence micrograph of a TR-DHPE labelled SLB after 1 week of cell culture. c) $D$-values presented as mean $\pm \operatorname{SD}(n=3)$. 


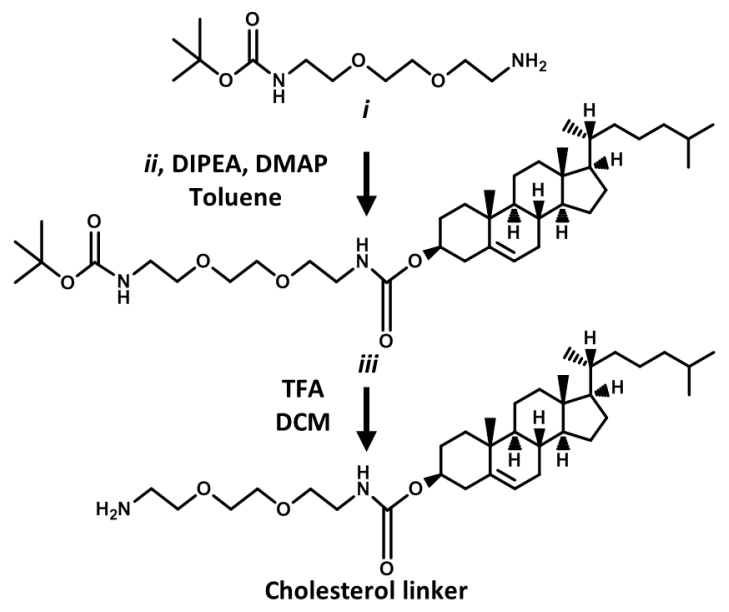

Figure 59: Cholesterol linker synthesis; scheme showing the 3-step synthesis of the amineterminated ethylene glycol spaced cholesterol linker.

a)

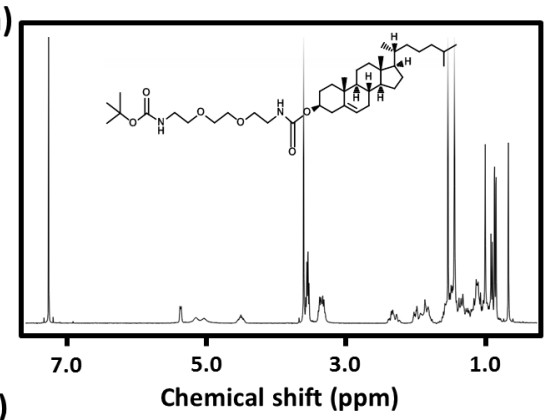

b)

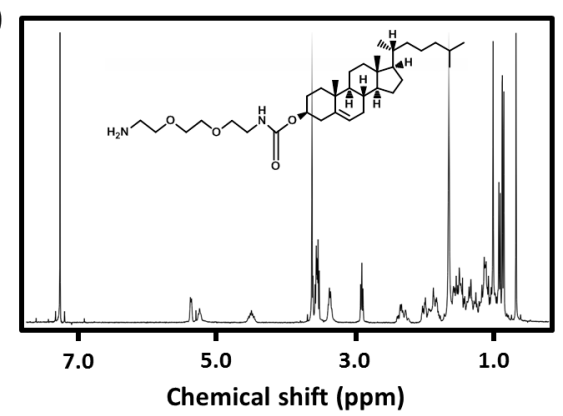

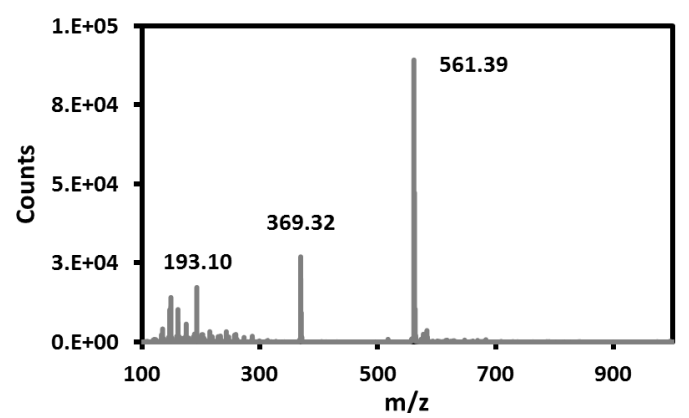

Figure S10: Characterization of the Cholesterol linker with ${ }^{1} \mathrm{H}$ NMR and TOF-ESI: a) ${ }^{1} \mathrm{H}$ NMR spectra of compound ii in $\mathrm{CDCl}_{3} ; 5.37(\mathrm{t}, 1 \mathrm{H}$, olefinic $\mathrm{H}), 5.25(\mathrm{t}, 1 \mathrm{H}, \mathrm{NH}), 5.1(\mathrm{t}, 1 \mathrm{H} \mathrm{NH}), 4.5(\mathrm{~m}, 1 \mathrm{H}, \mathrm{O}-\mathrm{CH}$ cholesterol), $3.61\left(\mathrm{~s}, 4 \mathrm{H}, \mathrm{O}-\mathrm{CH}_{2}-\mathrm{CH}_{2}-\mathrm{O}\right), 3.55\left(\mathrm{~m}, 4 \mathrm{H}, 2 \times \mathrm{O}-\mathrm{CH}_{2}\right), 3.37\left(\mathrm{~m}, 4 \mathrm{H}, 2 \times \mathrm{CH}_{2}\right.$ in $\left.\mathrm{CH}_{2} \mathrm{NH}\right), 2.5-$ $0.87\left(34 \mathrm{H}\right.$, cholesterol), 1.5 (s, 9H tert BOC), $0.86\left(\mathrm{~d}, 6 \mathrm{H} 2 \times \mathrm{CH}_{3}\right.$ cholesterol), $0.68\left(\mathrm{~s}, 3 \mathrm{H}, 1 \times \mathrm{CH}_{3}\right.$ cholesterol) b) ${ }^{1} \mathrm{H}$ NMR spectra compound iii in $\mathrm{CDCl}_{3} ; 5.37$ (t, $1 \mathrm{H}$, olefinic $\left.\mathrm{H}\right), 5.25(\mathrm{t}, 1 \mathrm{H} \mathrm{NH}), 4.5(\mathrm{~m}$, $1 \mathrm{H}, \mathrm{O}-\mathrm{CH}$ cholesterol), $3.61\left(\mathrm{~s}, 4 \mathrm{H}, \mathrm{O}-\mathrm{CH}_{2}-\mathrm{CH}_{2}-\mathrm{O}\right), 3.56\left(\mathrm{~m}, 4 \mathrm{H}, 2 \times \mathrm{XOCH}_{2}\right), 3.52\left(\mathrm{t}, 2 \mathrm{H}, \mathrm{NH}_{2}\right), 3.37(\mathrm{~m}, 2 \mathrm{H}$, $\mathrm{CH}_{2}$ in $\mathrm{CH}_{2} \mathrm{NH}$ ), 2.89 (t, $2 \mathrm{H}, \mathrm{CH}_{2} \mathrm{NH}_{2}$ ), 2.5-0.87 (34H, cholesterol), 0.86 (d, $6 \mathrm{H}, 2 \times \mathrm{CH}_{3}$ cholesterol), 0.68 (d, $3 \mathrm{H}, 1 \times \mathrm{CH}_{3}$ cholesterol). c) ToF-ESI spectra of compound iii, the cholesterol linker, obtained with cone voltages of $10-100 \mathrm{~V}, \mathrm{~m} / \mathrm{z} 561[\mathrm{M}+\mathrm{H}]+, 193$ and 369 mass abducts of compound iii. 
a)

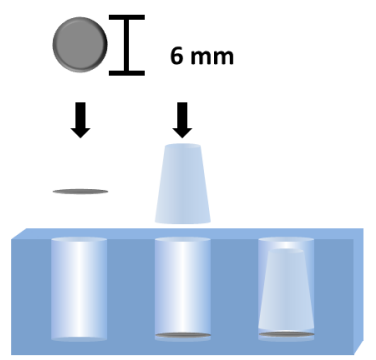

b)

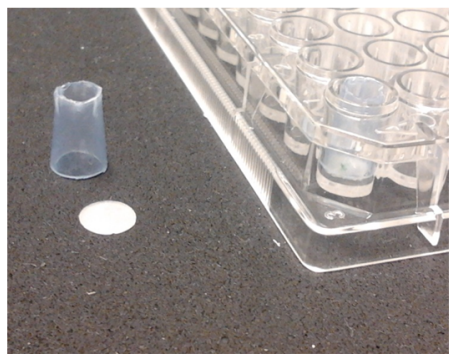

Figure S11: Cell culture on BSLBs prepared on PCL samples; a) schematic of sample mounting and $\mathbf{b}$ ) photograph of a cholesterol-modified PCL disk and cut pipet tip fix the samples. 
Chapter 7

\author{
Epilogue
}


In the present work, we studied new applications of supported lipid bilayers (SLBs). Although the technology has been described by McConnell and co-workers in the mideighties of the last century, ${ }^{1,2}$ the use of SLBs has evolved ever since.

\section{1 $\mu$ SLB electrophoresis}

In Chapter 2, we described a method that used several hundreds of millivolts to achieve electrophoretic separation of charged molecules within the SLBs. This achievement was made possible by including a sacrificial electrochemical reaction. ${ }^{3}$ We believe that the use of a sacrificial electrochemical reaction to mitigate water electrolysis is applicable to other forms of electrophoresis as well. For example, the addition of an electro-active compound has been used to supress bubble formation at the electrodes, ${ }^{4}$ but could also be used in alternating current (AC) SLB applications pioneered by Evans and co-workers. ${ }^{5}$ Although the proposed $\mu S L B$ electrophoresis device could be made to run on battery power and make use of capillary action for fluid exchange, the detection of analytes within such a nano-analytical device deserves considerable attention. Also, the integration with a fluidic system, as described by Höök and co-workers, ${ }^{6}$ could allow for the preparation of cellderived SLBs of which the components are separated in situ by an electric field. When the latter step is combined with hydrodynamic flow, ${ }^{7}$ 2D separation based on hydrodynamic volume and electrophoretic mobility is within reach. In addition, including embedded electrodes around the pin hole of 'black lipid membranes', 8 enables electrophoresis in free-standing SLBs.

\subsection{Locked-in gradients}

In Chapters 3 and 4, locked-in SLB gradients of various functional groups is described. ${ }^{9}$ The inherent non-fouling nature of SLBs that is retained to a large extent in these gel-state systems, allows for the study of e.g. binding events of fouling moieties. For example, proteins and cells (eukaryotic and prokaryotic) have the tendency to non-specifically adsorb to commonly used plastics and glasses. As a consequence, they often require blocking steps or chemical modification of their surfaces to facilitate specific interactions. The locked-in gradients are a more facile method to control ligand density and fouling. Apart from their use in quantitative glycomics (Chapter 4), combining model binding systems, such as cyclodextrin or cucurbituril systems for the study of supramolecular multivalent interactions, ${ }^{10}$ with locked-in gradients offer unique possibilities. One can imagine the generation of a host molecule gradient in low $T_{m}$ gel-state SLBs and the binding of a multivalent guest molecule to it. Upon local heating of the gradient, by e.g. a laser, a gel-to-liquid transition would occur allowing for the study of lateral dynamicity on multivalent binding at a predefined surface density of the host molecules. Moreover, embedding two functional groups with opposite charge during gradient formation, enables the generation of counter gradients. In addition, a variety of membrane-protein 
interactions could be investigated as well, such as $2 \mathrm{D}$ crystal formation of Annexin $\mathrm{V}$ and the binding and aggregate formation $\alpha$-Synuclein. ${ }^{11,12}$ In these cases, the interaction as a function of surface density of functional groups can be determined in both gel and liquidstate SLBs. The latter would be made possible upon rapid heating of the gradient and monitoring its recovery. In this case, a gradient of functional groups in a mobile SLB is generated, albeit with limited temporal stability.

\subsection{Directing cell fate using SLBs}

In Chapters 5 and 6, SLBs modified with cell adhesive peptides facilitated specific cell adhesion to its surface of both primary and stem cells. The versatility of SLBs in cell culture is reflected by the ease of which the density and mobility of functional groups can be tuned. Apart the well-studied ligand spacing, ${ }^{13-15}$ our research showed that lateral dynamicity affects cells dramatically. In the case of mesenchymal stem cell (MSC) differentiation, research into substrate stiffness and extracellular matrix (ECM) flexibility demonstrated the importance of the cell's mechanical environment and traction force generation. ${ }^{16-18}$ As was recently highlighted by Eyckmans and Chen, materials that have tailored dynamic substrate mechanics could be better suited to promote stem cell differentiation or maintain their phenotype. ${ }^{19}$ In this regard, controlling the lateral mobility of ligands within the SLB seem to fulfil these needs. ${ }^{20}$ Here, two aspects are of importance namely, the (1) mechanical dosing and (2) traction force generation. (1) Mechanical dosing relates to the time a cell experiences a given mechanical environment. In the case of SLBs, this is intimately controlled by ligand mobility. Choosing the lipids wisely and carefully controlling the cell culture temperature, one gains temporal control of ligand mobility. ${ }^{21}$ As a consequence, (2) the mechanical environment sensed by the cells can be controlled by SLB phase behaviour and potentially the choice of lipid anchors conjugated to ligands. However, the inherent air instability is a limiting factor in the use of SLBs. The work described in Chapter 6 proposed a method to stabilize the SLB, making e.g. in vivo applications more accessible. However, careful examination of the SLB integrity in time and stability of ligand-membrane association is paramount. We anticipate that these biomaterial supported lipid bilayers could be applied in in vivo applications such as vascular and orthopaedic medical implants, ex vivo applications such as ophthalmic and biosensor medical devices, and in research e.g. by coating of consumables such as cell culture plastics, screening and fluidic devices. ${ }^{22}$ Apart from the benefits SLBs offer ( $a$ priori), one could envision including small molecule drugs within the hydrophobic core of the lipid bilayer. 


\subsection{SLBs and supramolecular assemblies}

a)

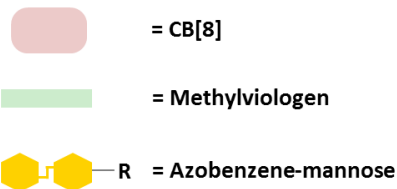

b)
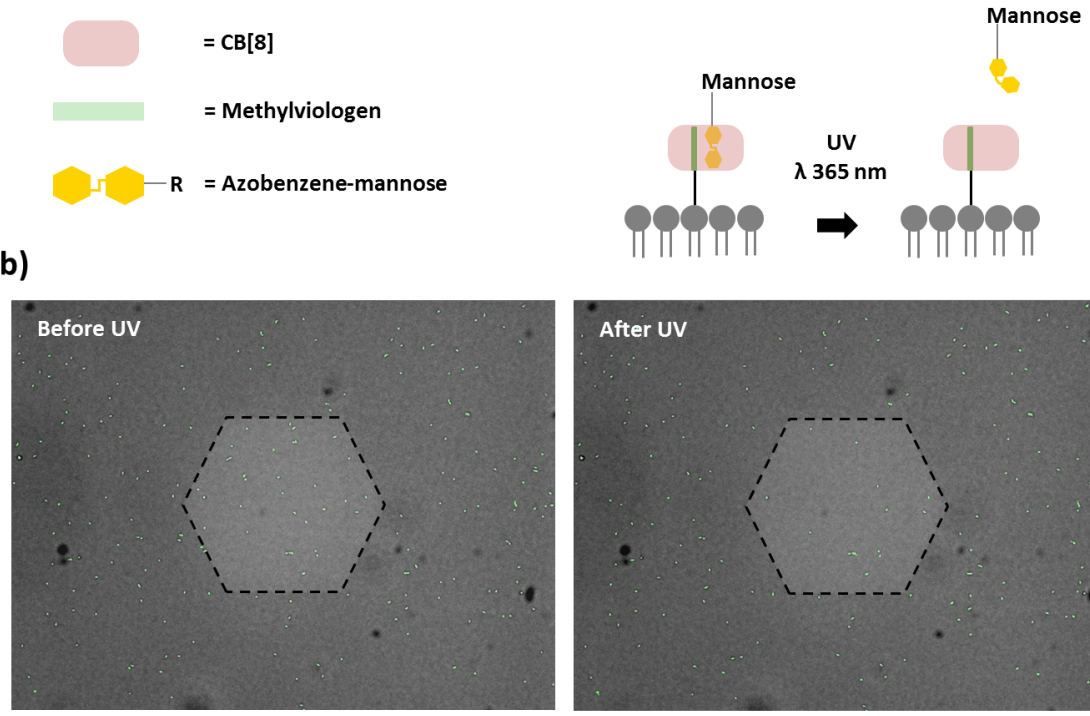

c)

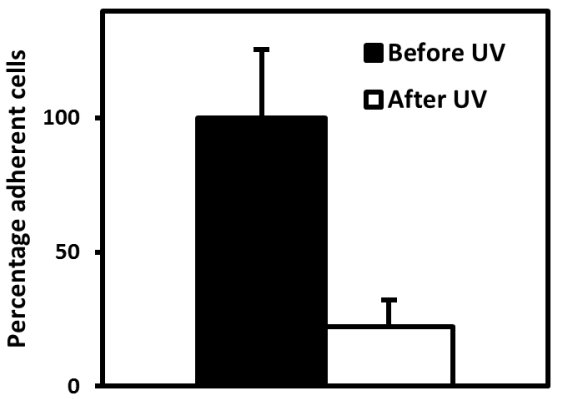

Figure 7.1: Supramolecular release of cells from SLBs; a) liquid-state SLB were modified with methylviologen and incubated with $\mathrm{CB}[8]$ and azobenzene-mannose. b) Upon incubation with $\mathrm{ORN}$ 178 bacteria (Green), specific binding to the ternay complex was observed. UV illumination with a partially closed field diaphragm resulted in c) selective removal of cells from the surface. Data presented as mean $\pm S D(n=4)$.

Traditionally, dynamic supramolecular systems such as cucurbiturils are studied on selfassembled monolayers on gold. Even though highly dynamic and switchable systems can be made, they lack in in-plane dynamicity. ${ }^{23,24}$ In vivo, in-plane dynamicity has been implicated to be of great importance in e.g. the formation of the immunological synapse. ${ }^{25}$ Current efforts within our group aim at combining supramolecular chemistry with SLBs to explore the impact of lateral dynamicity on ternary complex formation between guest 
molecules and host cucurbit[8]urils (CB[8]). In this case, the non-fouling nature of the SLB helps to promote specific interaction and enables the study of multivalent interactions. Towards this end, initial experiments demonstrate that it is possible to fabricate SLBS including the ternary methylviologen, $\mathrm{CB}[8]$ and azobenzene complex as given in Fig 7.1. The methylviologen is coupled to the SLB similar to the strategy in Chapter 4. The azobenzene in this experiment is derivatized with mannose to facilitate binding to cells. The azobenzene can undergo a trans-to-cis isomerization upon UV irradiation, resulting in its release from the ternary complex. Cells bound to mannose will be released as well. In the future, this would allow us to mimic clustering of synthetic receptors in the SLB using supramolecular assemblies. These cell membrane receptor analogues would further our understanding of receptor-ligand binding and could pave the way to new drug developments.

After more than thirty years, SLBs stood the test of time with exciting new developments such as the combination with block copolymer micelle nanolithography, ${ }^{26}$ nanoparticle

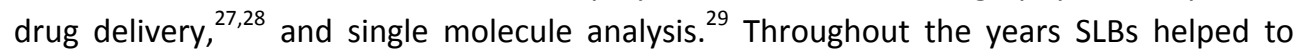
elucidate membrane-associated processes in e.g. cell adhesion ${ }^{20,30,31}$ and cancer biology. ${ }^{32,33}$ The work presented in this thesis could contribute to the development of novel bio-analytical applications and cell culture devices for directing cell fate in vitro and in vivo.

\subsection{References}

1 Brian, A. A.; McConnell, H. M. Proc. Natl. Acad. Sci. USA 1984, 81, 6159-6163.

2 Tamm, L. K.; Mcconnell, H. M. Biophys. J. 1985, 47, 105-113.

3 van Weerd, J.; Krabbenborg, S. O.; Eijkel, J.; Karperien, M.; Huskens, J.; Jonkheijm, P. J. Am. Chem. Soc. 2014, 136, 100-103.

4 Kohlheyer, D.; Eijkel, J. C. T.; Schlautmann, S.; van den Berg, A.; Schasfoort, R. B. M. Anal. Chem. 2008, 80, 4111-4118.

5 Bao, P.; Cheetham, M. R.; Roth, J. S.; Blakeston, A. C.; Bushby, R. J.; Evans, S. D. Anal. Chem. 2012, 84, 10702-10707.

6 Simonsson, L.; Gunnarsson, A.; Wallin, P.; Jonsson, P.; Hook, F. J. Am. Chem. Soc. 2011, 133, 1402714032.

7 Johansson, B.; Olsson, T.; Jonsson, P.; Hook, F. Soft Matter 2013, 9, 9414-9419.

8 Stimberg, V. C.; Bomer, J. G.; van Uitert, I.; van den Berg, A.; Le Gac, S. Small 2013, 9, 1076-1085.

9 Krabbenborg, S. O.; van Weerd, J.; Karperien, M.; Jonkheijm, P.; Huskens, J. ChemPhysChem 2014, 15, 3460-3465.

Perl, A.; Gomez-Casado, A.; Thompson, D.; Dam, H. H.; Jonkheijm, P.; Reinhoudt, D. N.; Huskens, J. Nat. Chem. 2011, 3, 317-322.

11 Bouter, A.; Gounou, C.; Berat, R.; Tan, S.; Gallois, B.; Granier, T.; d'Estaintot, B. L.; Poschl, E.; Brachvogel, B.; Brisson, A. R. Nat. Commun. 2011, 2. Hellstrand, E.; Grey, M.; Ainalem, M. L.; Ankner, J.; Forsyth, V. T.; Fragneto, G.; Haertlein, M.; Dauvergne, M. T.; Nilsson, H.; Brundin, P.; Linse, S.; Nylander, T.; Sparr, E. Acs Chem Neurosci 2013, 4, 1339-1351. Glass, R.; Moller, M.; Spatz, J. P. Nanotechnology 2003, 14, 1153-1160. 


7

Frith, J. E.; Mills, R. J.; Cooper-White, J. J. J. Cell Sci. 2012, 125, 317-327. Engler, A. J.; Sen, S.; Sweeney, H. L.; Discher, D. E. Cell 2006, 126, 677-689.

Trappmann, B.; Gautrot, J. E.; Connelly, J. T.; Strange, D. G. T.; Li, Y.; Oyen, M. L.; Cohen Stuart, M. A.; Boehm, H.; Li, B.; Vogel, V.; Spatz, J. P.; Watt, F. M.; Huck, W. T. S. Nat. Mater. 2012, 11, 642-649.

Vincent, L. G.; Engler, A. J. Nat. Mater. 2013, 12, 384-386.

Eyckmans, J.; Chen, C. S. Nat. Mater. 2014, 13, 542-543.

Yu, C.-h.; Rafiq, Nisha Bte M.; Krishnasamy, A.; Hartman, Kevin L.; Jones, Gareth E.; Bershadsky, Alexander D.; Sheetz, Michael P. Cell Reports 2013, 5, 1456-1468.

Andreasson-Ochsner, M.; Romano, G.; Hakanson, M.; Smith, M. L.; Leckband, D. E.; Textor, M.; Reimhult, E. Lab Chip 2011, 11, 2876-2883.

EUR pat., PCT EP2014060173, 2014.

An, Q.; Brinkmann, J.; Huskens, J.; Krabbenborg, S.; de Boer, J.; Jonkheijm, P. Angew. Chem. Int. Ed. 2012, 51, 12233-12237.

Brinkmann, J.; Cavatorta, E.; Sankaran, S.; Schmidt, B.; van Weerd, J.; Jonkheijm, P. Chem. Soc. Rev. 2014, 43, 4449-4469.

Manz, B. N.; Jackson, B. L.; Petit, R. S.; Dustin, M. L.; Groves, J. Proc. Natl. Acad. Sci. USA 2011, 108, 9089-9094.

Lohmuller, T.; Triffo, S.; O'Donoghue, G. P.; Xu, Q.; Coyle, M. P.; Groves, J. T. Nano Lett. 2011, 11, 4912 4918.

Ashley, C. E.; Carnes, E. C.; Phillips, G. K.; Padilla, D.; Durfee, P. N.; Brown, P. A.; Hanna, T. N.; Liu, J.; Phillips, B.; Carter, M. B.; Carroll, N. J.; Jiang, X.; Dunphy, D. R.; Willman, C. L.; Petsev, D. N.; Evans, D. G.; Parikh, A. N.; Chackerian, B.; Wharton, W.; Peabody, D. S.; Brinker, C. J. Nat. Mater. 2011, 10, 389-397.

\section{Shen, S.; Kendall, E.; Oliver, A.; Ngassam, V.; Hu, D.; Parikh, A. N. Soft Matter 2011, 1001-1005.}

Iversen, L.; Tu, H. L.; Lin, W. C.; Christensen, S. M.; Abel, S. M.; Iwig, J.; Wu, H. J.; Gureasko, J.; Rhodes, C.; Petit, R. S.; Hansen, S. D.; Thill, P.; Yu, C. H.; Stamou, D.; Chakraborty, A. K.; Kuriyan, J.; Groves, J. T. Science 2014, 345, 50-54. Yu, C. H.; Law, J. B. K.; Suryana, M.; Low, H. Y.; Sheetz, M. P. Proc. Natl. Acad. Sci. USA 2011, 108, 2058520590.

Yu, C. H.; Luo, W. W.; Sheetz, M. P. Cell Adhes. Migr. 2012, 6, 280-284.

Salaita, K.; Nair, P. M.; Petit, R. S.; Neve, R. M.; Das, D.; Gray, J. W.; Groves, J. T. Science 2010, 327, $1380-$ 1385.

Xu, Q.; Lin, W. C.; Petit, R. S.; Groves, J. T. Biophys. J. 2011, 101, 2731-2739. 


\section{Summary}

Studying and steering cell behaviour on artificial surfaces is challenged by the correct presentation of ligands and interaction with cells. In this respect, dynamic biological synthetic architectures have been proposed that rely on supramolecular assembly, fabrication techniques, adaptive chemistry and modern molecular biology. Cell membrane mimics such as supported lipid bilayers (SLBs) offer unique possibilities in this field. For example, ligands that are displayed on SLBs can move in 2D while prohibiting non-specific interaction with proteins and cells. Currently, SLBs are used for surfaces of biosensors, for the separation medium of membrane components and as cell culture platforms to mimic the extracellular matrix and cell-cell contacts.

The aim of the work described in the thesis is to explore novel biomedical applications of SLBs with implications for (1) membrane separation, (2) surface gradient formation and (3) artificial cell interfaces for in vivo use. (1) Membrane separation by means of electrophoresis suffer from large applied potentials that prevent down-scaling to lowpower nano-analytical devices and can cause damage to the SLB. In this part of the work a chip-based system was fabricated in a process that consumes only 100 s of millivolts. This achievement was made possible through the addition of a sacrificial electrochemical reaction during SLB electrophoresis. (2) Concentration gradients of charged analytes within the SLB-based gradients do not display temporal stability and can therefore not be easily used as e.g. a continuous gradient for the study of binding events. In this part of the work the SLB electrophoresis system was used to generate surface gradients of charged functional groups in gel-state SLBs. These gradients proved stable in time conveniently at room temperature and could be modified with various bioactive compounds such as mannose to study the binding of E. coli under relevant physiological shear stresses. (3) Even though SLBs have been used extensively in vitro, their application in vivo has been limited due to the air instability of these layers. In this part of the work the interligand spacing and lateral mobility of cell adhesive ligands was varied in SLBs and studied in cell culture. Chondrocyte and preosteoblastic cell behaviour could be tuned in terms of cell adhesion, cell spreading, cytoskeletal organization and the extent of matrix deposition. Ultimately, the preparation of a cholesterol-modified biopolymer allowed us to prepare air-stable biomaterial SLB, which were equally non-fouling and, when bioactive ligands were included, able to steer mesenchymal stem cell fate.

In summary, the lateral mobility of lipids and ligands in SLBs can be exploited for gradient formation and tuning traction force generation by cells. Besides their use as bio-analytical tools, we foresee air-stable SLBs being adopted in Tissue Engineering for improving the host-biomaterial response in the case of implant technology. For example, biomaterial SLBs could be used to promote host integration of medical devices. 


\section{Samenvatting}

Het bestuderen en het beïnvloeden van cellen op artificiële oppervlakten wordt bemoeilijkt door de presentatie van liganden en hun interactie met cellen. Om dit te verbeteren worden synthetische en dynamische biologische structuren voorgesteld die gebruik maken van supramoleculaire assemblage, fabricage technieken, chemie en moderne moleculaire cel biologie. Analoga van celmembranen zoals ondersteunde lipide dubbellagen (OLDs) bieden unieke mogelijkheden in dit onderzoeksveld. Liganden die gepresenteerd worden op OLDs vertonen bewegelijkheid in 2D terwijl ze geen oppervlakte vervuiling toelaten van eiwitten of cellen. OLDs worden veelal gebruikt als oppervlakte in biosensoren, als het scheidingsmedium van cel membraan componenten en in cel kweek waarin de extracellulaire matrix en cel-cel contacten kunnen worden nagebootst.

Het doel van het werk dat is beschreven in dit proefschrift is het verkennen van nieuwe toepassingsgebieden van OLDs zoals (1) membraan scheiding, (2) oppervlakte gradiënten en (3) artificieel cel oppervlak in vivo. (1) Membraanscheiding met elektroforese wordt gehinderd door de hoge potentiaalverschillen die noodzakelijk zijn, hetgeen de miniaturisatie in de weg staat en schade kan veroorzaken aan de OLD. In dit deel van het onderzoek is een OLD elektroforese chip ontwikkeld die door gebruik van een elektrochemische reactie slechts honderden millivolts vereist. (2) Concentratie gradiënten van geladen moleculen in OLDs zijn niet stabiel in de tijd met als gevolg dat ze ongeschikt zijn voor het eenvoudig uitvoeren van bindingsstudies. In dit deel van het onderzoek is het OLD elektroforese systeem gebruikt om oppervlakte gradiënten te maken van geladen functionele groepen in gel-staat OLDs. De gradiënten bleken stabiel in de tijd en konden gemodificeerd worden met verschillende stoffen zoals mannose, welke gebruikt is om het bindingsgedrag van $E$. coli te bestuderen onder relevante shear stress. (3) Ondanks het feit dat OLDs veel in vitro toegepast worden, is hun gebruik in vivo beperkt, mede door hun luchtinstabiliteit. In dit deel van het onderzoek is gekeken naar het effect van de dichtheid en bewegelijkheid van cel-bindende liganden in OLDs tijdens celkweek. Het gedrag van chondrocyten en preosteoblastische cellen kon beïnvloedt worden in termen van cel adhesie en spreiding, organisatie van het cytoskelet en de mate van matrix uitscheiding. Uiteindelijk heeft de ontwikkeling van een cholesterol-gemodificeerd biopolymeer ertoe geleidt dat luchtstabiele biomateriaal OLDs gevormd konden worden, die grote mate van bioinert gedrag vertoonde en na het invoegen van bioactive liganden het gedrag van mesenchymale stamcellen konden sturen.

Samenvattend blijkt dat de bewegelijkheid van lipiden en liganden in OLDs voor gradiënten en het beïnvloeden van cel tractie toegepast kan worden. Naast hun gebruik als bio-analytische tools, zien wij grote mogelijkheden in weefselhersteltechnologie voor het aanbrengen van luchtstabiele OLDs op biomaterialen om de integratie van medische hulpmiddelen met weefsel te verbeteren. 


\section{Risk Analysis \& Technology Assessment ${ }^{1}$}

The risk analysis \& technology assessment (RATA) innovation cycle helps us to identify, during the different stages of innovation, what risks are involved and what needs to be taken into consideration with the emergence of new technology. The innovation cycle describes the start of the invention with research, translation into innovation in industry, diffusion into society and eventually authorization and regulation by policy makers. In particular when a clinical application is envisioned, researchers must be aware of the risks of their technology for human health and of course the environment. Moreover, the ethical implications of their research in society could have far reaching effects. In this regard, the work described in Chapter 6 could have broad implications in the aforementioned fields.

To mitigate the risks involved in the technology described in Chapter 6, we choose to make use of a natural system. The proposed coating technology is based on lipid molecules that are found in nature and are present in the membrane of human cells. As a consequence, the use of these molecules is associated with very low health risks for the end-user. For example, drug delivery systems using similar molecules have been described, used in the clinic and extensively tested and approved by the food and drug administration (FDA). In addition, the fabrication of these molecules is performed under current good manufacturing practice (cGMP) thus ensuring the quality of these molecules. The biomaterials that we coated in Chapter 6 are well described, and in the case of polycaprolactone (PCL), is FDA approved as well. However, our coating process requires a plasma preconditioning step and modification with a cholesterol-based linker molecule of the underlying biomaterial. Although, plasma preconditioning of biomaterials intended for in vivo use is associated with low health risks, the modification with the cholesterol molecule could have adverse effects. Since the biomaterials can degrade in time in the human body, it is anticipated that the cholesterol molecule will become available to the host tissue. Therefore cytotoxicity, clearance and degradation (e.g. hydrolytic or metabolic) must be assessed. In addition, the molecule is prepared in a research laboratory and does not meet cGMP yet. During our experiments on human cells, we did not observe any cytotoxicity induced by our coating. However, a dedicated study in silico (e.g. European inventory of existing commercial chemical substances, EINECS and registration, evaluation and authorization of chemicals, $\mathrm{REACH}$ databases) and in vitro (e.g. degradation rate and toxic potency) must be performed. Environmental effects can

\footnotetext{
${ }^{1}$ Awareness of RATA by researchers at an early stage is crucial for innovation success. The RATA initiative in the public-private partnership of NanoNextNL provides researchers with the tools and the knowledge-base for risk analysis and technology assessment.
} 
be judged by the risk characterization ratio between the predicted environmental concentration (PEC) and predicted no effect concentration (PNEC). Ideally this should be performed for the intact molecule, its degradation products and hazardous waste during synthesis, particularly important for industry and large scale production.

In society we foresee two aspects that deserve attention namely; technology acceptance and clinical implications. We believe that the very nature of our coating method, comprising of natural compounds, would be enticed by the public. Given the current societal trends in e.g. the biological food sector and sustainable energy, our biomedical coating developments align well with mind-sets of the general public towards more 'bio' and 'friendly' approaches. Under the premise that the coating is successfully implemented on biomedical devices and improves their function, our coating could make the use of biomaterials in vivo more commonplace due to their increased success rate. In the case of orthopaedic implants, this could lead to a reduction in revision surgery and improved joint function. This in turn could have implications for the elderly that regain their mobility and become less dependent on health care institutions for daily needs.

This development could lead to changes in legislation and health care costs. On the one hand, clinical intervention and implantation of a costly biomedical device might than be preferred over cheaper but prolonged aid and therapy of the patients by health insurance agencies and governments. On the other hand when such medical devices are not reimbursed, it could widen the gap between poor and rich people.

Even though we are in the initial stage of the innovation cycle, RATA evaluating of our technology reveals great commercial potential with a positive RATA outlook. Although certain risks are identified, yet not well understand, their long-term technical and societal impact must be evaluated during the course of future technology development. 


\section{Acknowledgements}

At the close of my PhD, I would like to thank all the people that have supported and helped me during the past 4 years. No words can express my gratitude to all that have been involved. I will do my best to mention each and every one of you.

I would like to start off by thanking Pascal and Marcel for giving me the opportunity to pursue my PhD. I very much enjoyed the open atmosphere you both provided. Especially the freedom to explore different topics has helped me to developed my scientific skills. I cherish the discussions we had and how they led to new insights and ideas. Marcel, I want to thank you for presenting me with this opportunity at the end of my master assignment. Your support and trust was and still is very important to me. Pascal, I admire the way you supervised me the past years. I could always come by if I had questions or to brainstorm on a certain problem. This reflects on your group as well, everybody is willing to help each other and show a keen interest in the various projects. You helped me grow during my PhD both as a scientist and as a person. I wish you the best together with Dodo and Jasmijn. Marcel and Pascal, I am looking forward to our new adventure.

A special thanks to Jurriaan who was heavily involved during the first years of my PhD and later on with the gradient work in Chapters 2 and 3. Your to-the-point discussions always resulted in a clear plan of action. Jan, your expertise and input on the work presented in Chapter 2 was invaluable and led to the success of the project. A special thanks as well to Paul for giving me the opportunity to visit your lab at the end of my PhD and collaborate with Matthew. Although a brief period, the results obtained laid the foundation for future collaboration.

When I started my PhD as a novice in chemistry and attempting to synthesize my first compound, Carlo was there to lend me a helping hand. You acquainted me with various techniques and in time a lot of hocus-pocus became clear, thank you. I wish you and Mariska all the best for the future. Continuing my chemistry adventure led me to do peptide synthesis. Fortunately, my saving Angel was there to show me the ins and outs of the Syro. I enjoyed working together with you and the best of luck with your academic career in China. If for some reason you consider a career change, I would put being a race driver on the bottom of the list. Jordi, to some extent were 'bound' by the VHH. I recall one of the first times we met in Langezijds where we shared our mutual frustration of these molecules. You helped me find my way in the lab and assisted me with techniques such as HPLC. You were always willing to help and discuss various things, thank you. All the best to you and Danny. I still have muscle ache though for chasing and being chased 
by that horse for your PhD movie. Sven, my super glue and scotch tape partner. Even though our initial collaboration resulted in a hideous blob of glue, tape, PDMS and a chip, we did manage in a short period of time to create a robust device that we are still using for different applications. I very much enjoyed our work together and I am proud of what we achieved in such a short period of time, that resulted in Chapters 2 and 3 . I also enjoyed being your paranymph which allowed me to expand on my Hobbit skills. I wish you and Maaike and all the best for the future. Of course, it was only a matter of time that supramolecular host-guest chemistry, used in Pascal's group, was combined with the SLBs. Shirish, we started off trying to assemble supramolecular complexes on the bilayer. Funny enough, the initial experiments resulted in a completely different study using the gradients in Chapter 4. Our continued collaboration is proven to be highly successful and most importantly you taught me the bacteria song. Good luck with finalizing your PhD and I wish you and Jenny all the best. Jens, the synthesis king, thank you for your support during the bacterial gradient project (Chapter 4) and our on-going research with Shirish on supramolecular assemblies on SLBs. Nicole, the idea was straightforward, namely growing chondrocytes on the bilayer, stimulate them with a peptide-growth factor complex and study their behaviour. However, at each step we had to overcome major hurdles. Even though we were not able to reach our end goal, we still got intriguing results that promote further investigations, which resulted in Chapter 5. I would also like to acknowledge the support of Percuros B.V. In particular Alan and Karien for giving me the opportunity to work in your labs and for your support.

I had the pleasure of supervising various students of which their work was of utmost importance to further our understanding of SLBs and their application. I want to thank Aryan, Johan, Vincent, Dirk-Jan and Stan. All of you grew during your time in the group and I appreciate the chance I got to supervise all of you.

Aditya, you were one of the few people studying lipid bilayers at our university. I enjoyed the discussions we had and I wish you all the best at AMOLF for the last part of your PhD. Aldo, you visited our labs for a crash-course in SLB formation and characterization on your sensor devices. I am very pleased that the experiments went like a charm and that your research is gaining a foothold. All the best for the future and with finalizing your PhD.

I want to thank the staff of the SaNS cluster Natalie, Wim, Jeroen, Tibor and Melissa for their support and for providing a nice working atmosphere. All of the work would not have been possible without the support of the technical staff of the SaNS cluster, the Bionanolab and the DBE group. I want to thank Marcel, Richard, Regine, Bianca, Tieme, Irene, Ine and Jacqueline for making the labs operate as a well-oiled machine. Richard and Marcel you were always there assist me in any way or form, thank you. Also, it was nice to 
share my car tinkering passion. A special thanks to the secretaries Nicole, Izabel and Ingrid as well for their unwavering support.

Dear paranymphs, Stan and Rick, thank you for your help with the final preparations of my PhD. Rick, I am still amazed of the shear amount of frikandellen you can eat. I believe you might have discovered the fountain of youth with your diet. I want to thank you for the fun times we had. If for some reason science doesn't work out, we could consider a movie career instead. Good luck with the 'laatste loodjes' of the PhD and I wish you all the best with your spouse Alex. On the other end of the diet spectrum we have the paprika eating Stan. Stan, you were my first student during the PhD. Together we had a lot of fun and you were able to obtain very nice results. I recall one memorable moment on the lab where we did peptide synthesis together with Pascal and Dodo. I think you agree that was quite interesting. Stan, you can be dubbed the bonified SaNS 'job hopper'. Starting within Pascal's group for your bachelors, your masters in Jurriaan's group and your PhD in Jeroen's group. I am sure it will be a 'great success'.

I want to express a special thanks to all the people of Pascal's group that I had the privilege of working and socializing with. I enjoyed the time we had, Dodo, Emanuela, Jenny, Shirish, Tushar, Tom, Erhan, Bettina, João, Aryan, Johan, Gülistan, Mark \& Mark, Wies, Maike, Nora, Nicole, Angel, Jordi, and Jens, thank you and I wish you all the best.

Of course I want to mention my 'room mates'. When I started in Langezijds we, Carmen, Melani and myself, were stowed away in a drafty office to write our literature report. Carmen, you also reached the finish line and will be defending soon. I enjoyed the time we have had and our fun discussions. I wish you, Jan, and the baby all the best and good luck with the final preparations. Melani and Alberto, you finished your PhDs some time ago now and are all settled in, good luck in the future. Upon moving to the new Carré building, room quality significantly improved in the form of a more or less private office next to lab 5. Rik, Anne and Yujie, although our private office was short-lived I sincerely enjoyed the time we had. Rik, one of our first endeavours was the development of an espresso machine with a cloak. Even though the machine itself remained undetected for quite some time, I do feel the smell and the steam it put out gave it away. Also, thank you for rekindling my love for Scooter whilst being your paranymph. Rik and Rianne, I wish you both all the best. Pieter, I am still not sure if you decided to be German or Dutch? Many thanks for the fun times we had and good luck with your career at BASF. Best wishes to you and Aike. Raluca, the paranymph queen, I had a lot of fun with you around. Especially while preparing for Jordi's defence. 
I want to thank my current and formers colleges from MnF, BNT, DBE and more, Rajesh, Roberto, Laura, Raquel, Maarten, Martijn, Anna, Fabian, Deniz, Chengfen, Benjamin, Alejandro, Kim, Oktay, Roald, Balachander, Andrea, Jealemy, Mudassir, Nicolai, Piotr, Oya, Sarah, Timon, Vijay, Rindia, He, Alexander, Andreas, Janneke, Wouter, Elahe, Jeroen, Sanne, Supitchaya, Supaporn, Ajie, Wilfred, Emilie, Andrew, David, I am humbled to have worked with so many great people.

Als laatste wil ik mijn familie en vrienden bedanken voor hun steun de afgelopen jaren. Met een speciale dank aan mijn ouders, broer, schoonzus en natuurlijk mijn nichtje. Tot slot wil ik mijn verloofde Ariëlla bedanken. Ariëlla, jij staat altijd voor mij klaar en steunt mij volledig. Ik ben intens gelukkig dat wij in goede en in slechte tijden op elkaar terug kunnen vallen. Mijn liefde voor jou is onvoorwaardelijk en ik kijk uit naar een mooie toekomst samen.

Thank you all from the bottom of my heart.

Jasper

Enschede, December, 2014 


\section{Curriculum Vitea}

Jasper van Weerd was born in Almelo on the 24th of October 1985. From 2003 till 2007 he studied 'Biology and Medical Laboratory Research' at the Saxion University of Applied Sciences. In July 2007 he obtained his 'Bachelor of Applied Science' degree. His bachelor dissertation was concerned with the development of a rapid DNA detection method for airborne biological agents. This research was done at ZebraBioscience B.V. under supervision of Dr. R.P. van Weeghel.

In September 2007 Jasper continued his studies starting a 'Biomedical Engineering' master, specializing in 'Molecular, Cellular and Tissue Engineering', at the University of Twente. In October 2010 he received his master's degree after studying the use of heavy chain antibodies as a non-covalent, bio-active biomaterial coating for enhanced tissue repair. His master thesis research was carried out at the 'Cellular Architecture and Dynamics group' at the Utrecht University, 'Polymer Chemistry and BioMaterials group' and the 'Tissue Regeneration department' at the University of Twente.

Parallel to his master study he was employed by ZebraBioscience B.V. (from 2008 till 2010) as a part-time researcher on the FP6 EU-project HIBISCUS (Hybrid Integrated Bio-Sensor Created by Ultrafast Laser Sources). Here, he worked on various topics ranging from microfluidics, optics to genetic engineering for the design of a chip for high resolution and multiplexed DNA separation. This work was carried out in collaboration with B.V., Micronit B.V., Lionix B.V., the 'Integrated Optical Microsystems group' University of Twente and the Politechnico de Milano.

From October 2010 until October 2014 he was a PhD candidate under supervision of Prof. Dr. P. Jonkheijm of the 'Laboratory of Bioinspired Molecular Engineering' and Prof. Dr. H.B.J. Karperien of the 'Developmental BioEngineering group' at the University of Twente. The results of his PhD work are presented in this thesis. 


\section{List of publications}

Patents

J. van Weerd, M. Karperien and P. Jonkheijm.

PCT EP2014060173: "Process for the preparation of an object supporting a lipid bilayer"

Papers

12. S. Sankaran*, J. van Weerd*, J. Voskuhl, M. Karperien and P. Jonkheijm

"Triggered supramolecular binding and release of cells from supported lipid bilayers"

Manuscript in preparation

*Equal authorship

11. J. van Weerd, N. Georgi, K. de Rooij, M. Karperien and P. Jonkheijm

"Tuning cell adhesion, spreading and matrix formation of cells in vitro using SLBs"

Manuscript in preparation

10. J. van Weerd; M. Karperien and P. Jonkheijm

"Supported lipid bilayers for the generation of dynamic cell-material interfaces"

Manuscript in preparation

9. J. van Weerd*; S. Sankaran*; O. Roling; S. Sukas; B.J. Ravoo; M. Karperien and P. Jonkheijm

"Probing the threshold in bacteria-mannose recognition using SLB gradients"

Manuscript in preparation

*Equal authorship

8. J. van Weerd; J. Huskens; M. Karperien and P. Jonkheijm

"Biomaterial supported lipid bilayer as tuneable cell surface"

Manuscript in preparation

7. E.D. Rodrigues; S. Oliveira; I. Que; M. El Khattabi; J. van Weerd; E.B.M. Landman;

C.A. van Blitterswijk; C.W.G.M. Löwik; T. Verrips; A. Chan and M. Karperien

"Targeting bone with a single domain antibody binding hydroxyapatite"

Manuscript in preparation

6. J. Cabanas-Danés; E.D. Rodrigues; E. Landman; J. van Weerd; C.A. van Blitterswijk, T. Verrips, J. Huskens, M. Karperien and P. Jonkheijm

"A Supramolecular Host-Guest Carrier System for Growth Factors..."

J. Am. Chem. Soc. (2014) v.136, p.12675-12681 
5. S.O. Krabbenborg*; J. van Weerd*; M. Karperien; P. Jonkheijm and J. Huskens.

"Locked-in biomimetic surface gradients that are tuneable in size ..."

ChemPhysChem (2014) v.15, p.3460-3465

*Equal authorship

4. J. Brinkmann; E. Cavatorta; S. Sankaran; B. Schmidt; J. van Weerd and P. Jonkheijm. "About supramolecular systems for dynamically probing cells"

Chem. Soc. Rev. (2014) v.43, p.4449-4469

3. J. van Weerd*; S. O. Krabbenborg*; J. Eijkel, M. Karperien; J. Huskens and P. Jonkheijm.

"On-Chip electrophoresis in supported lipid bilayer membranes achieved..."

J. Am. Chem. Soc. (2014) v.136, p.100-103

*Equal authorship

3. C. Dongre; J. van Weerd; G.A.J. Besselink; R. Osellame; R. Martínez Vázquez; G. Cerullo; R. van Weeghel; H.H. van den Vlekkert; H.J.W.M. Hoekstra and M. Pollnau, "Modulation-frequency encoded multi-color fluorescent DNA analysis..." Lab Chip (2011) v.11, p.679-683

2. C. Dongre; J. van Weerd; R. van Weeghel; R. Martinez Vazquez; R. Osellame; G. Cerullo; M. Cretich; M. Chiari; H. J.W.M. Hoekstra and M. Pollnau.

"High-resolution electrophoretic separation and integrated-waveguid ..."

Electrophoresis (2010) v.31, p.2584-2588

1. C. Dongre; J. van Weerd; N. Bellini; R. Osellame; G. Cerullo; R. van Weeghel; H.J.W.M. Hoekstra and M. Pollnau.

"Dual-point dual-wavelength fluorescence monitoring of DNA separation..."

Biomed. Opt. Express (2010) v.1,p.729-735 
Conference contributions

J. van Weerd; S. Krabbenborg ; J. Huskens; M. Karperien and P. Jonkheijm. "Novel mimetic cell membranes: From in vitro towards in vivo applications" ACS conference, San Francisco (USA), 2014, SciMix presentation

J. van Weerd; S. Krabbenborg ; J. Huskens; M. Karperien and P. Jonkheijm. "Novel mimetic cell membranes: From in vitro towards in vivo applications" ACS conference, San Francisco (USA), 2014, Oral presentation

J. van Weerd; M. Karperien; J. Huskens and P. Jonkheijm.

"Biomaterial supported lipid bilayer: Tuneable cell surface for enhanced tissue repair" Frontiers in Synthetic Life, Nijmegen (NL), 2013, Poster presentation

J. van Weerd; S.O. Krabbenborg; M. Karperien; J. Huskens and P. Jonkheijm.

"Mimetic membranes systems: From in vitro to in vivo..."

NanoNextNL Beyond more theme day, Utrecht (NL), 2013, Oral presentation

J. van Weerd; S.O. Krabbenborg; M. Karperien; J. Huskens and P. Jonkheijm.

"Mimetic membranes systems: From in vitro to in vivo..."

$\mathrm{MESA}^{+}$day, University of Twente (NL), 2013, Oral presentation

J. van Weerd; M. Karperien; J. Huskens and P. Jonkheijm.

"Biomaterial supported lipid bilayer: Tuneable cell surface for enhanced tissue repair" COMS conference, University of Twente (NL), 2013, Stand/presentation

J. van Weerd; M. Karperien; J. Huskens and P. Jonkheijm.

"Biomaterial supported lipid bilayer: Tuneable cell surface for enhanced tissue repair" FEBS-ESF workshop, Sant Felui de Guixols (ESP), 2013, Poster presentation

J. van Weerd; M. Karperien; J. Huskens and P. Jonkheijm.

"Tuneable cell fate on biomaterial supported lipid bilayers"

BMM annual meeting, Ermelo (NL), 2013, Oral presentation

J. van Weerd; M. Karperien; J. Huskens and P. Jonkheijm.

"Biomaterial supported lipid bilayer: Tuneable cell surface for enhanced tissue repair" E-MRS spring meeting, Strasbourg, 2013 (FR), Oral presentation 
J. van Weerd; M. Karperien; J. Huskens and P. Jonkheijm.

"Biomaterial supported lipid bilayer as tuneable cell surface"

MicroNanoConference, Reehorst Ede (NL), 2012 Poster presentation

J. van Weerd; M. Karperien; J. Huskens and P. Jonkheijm.

"Biomaterial supported lipid bilayers as novel interface to living cells"

Work week seminar, Berlin (GER), 2012, Oral presentation

J. van Weerd; M. Karperien; J. Huskens and P. Jonkheijm.

"Tethered air-stable lipid bilayer platform as interfacial layer on polymeric..." Gordon Research Seminar and conferences, Bio interface science, 2012 Poster presentation

J. van Weerd; M. Karperien; J. Huskens and P. Jonkheijm.

"Interfaces to living cells"

MicroNanoConference, University of Twente (NL), 2011, Poster presentation 
